\title{
Ground-Water and Surface-Water Elevations in the Fairbanks International Airport Area, Alaska, 1990-94
}

By David V. Claar and Michael R. Lilly

U.S. GEOLOGICAL SURVEY

Open-File Report 95-382

Prepared in cooperation with the

ALASKA DEPARTMENT OF TRANSPORTATION

AND PUBLIC FACILITIES, FAIRBANKS INTERNATIONAL AIRPORT

Fairbanks, Alaska

1995 


\title{
U.S. DEPARTMENT OF THE INTERIOR \\ BRUCE BABBITT, Secretary
}

\author{
U.S. GEOLOGICAL SURVEY \\ Gordon P. Eaton, Director
}

For additional information write to:

District Chief

U.S. Geological Survey

4230 University Drive, Suite 201

Anchorage, AK 99508-4664
Copies of this report can be purchased from:

U.S. Geological Survey

Earth Science Information Center

Open-File Reports Section

Box 25425, MS 517

Denver Federal Center

Denver, CO 80225-0425 


\section{CONTENTS}

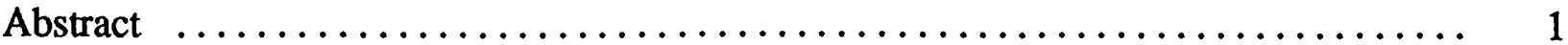

Introduction $\ldots \ldots \ldots \ldots \ldots \ldots \ldots \ldots \ldots \ldots \ldots \ldots \ldots \ldots \ldots \ldots, 1$

Hydrologic setting $\ldots \ldots \ldots \ldots \ldots \ldots \ldots \ldots \ldots \ldots \ldots \ldots \ldots \ldots \ldots \ldots, 1$

Ground-water sites.................................... 5

Surface-water sites..................................... 5

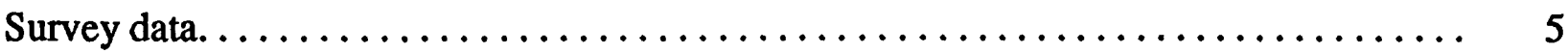

Ground-water and surface-water elevations $\ldots \ldots \ldots \ldots \ldots \ldots \ldots \ldots \ldots \ldots \ldots, 6$

References cited........................................... 6

Water-elevation tables and hydrographs $\ldots \ldots \ldots \ldots \ldots \ldots \ldots \ldots \ldots \ldots \ldots, \quad 9$

\section{PLATES [Plates in pocket]}

1. Map showing geohydrologic data-collection sites, Fairbanks International Airport study area.

2. Map showing geohydrologic data-collection sites in the former fire-training area, Fairbanks International Airport.

\section{FIGURES}

1. Map showing location of Fairbanks, Alaska, and the Fairbanks International

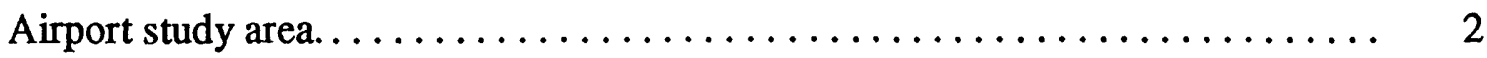

2. Hydrograph showing water-surface elevations of the Tanana and Chena Rivers at Fairbanks gaging stations for calendar years 1991 to $1994 \ldots \ldots \ldots \ldots \ldots$. 4

3. Diagram showing derivation of local number from the official rectangular subdivision of public lands.

\section{TABLE}

1. Ground- and surface-water data-collection sites, Fairbanks International Airport area. 


\section{CONVERSION FACTORS, VERTICAL DATUM, AND ABBREVIATIONS}

\begin{tabular}{rll}
\hline Multiply & By & To obtaln \\
foot (ft) & 0.3048 & meter \\
mile (mi) & 1.609 & kilometer \\
square mile $\left(\mathrm{mi}^{2}\right)$ & 2.59 & square kilometer \\
foot per second (ft/s) & 0.3048 & meter per second \\
gallon per minute (gal/min) & 0.06308 & liter per second \\
& & \\
\hline
\end{tabular}

\section{Vertical Datum:}

In this report, "sea level" refers to the National Geodetic Vertical Datum of 1929 (NVGD of 1929), a geodetic datum derived from a general adjustment of the first-order level nets of both the United States and Canada, formerly called Sea Level Datum of 1929.

\section{Horizontal Datum:}

The horizontal datum for all locations in this report is the North American Datum of 1927. Multiple reference marks were used from Alaska Department of Transportation and Public Facilities surveys. Global positioning survey instruments were also used to determine horizontal control points. 


\title{
Ground-Water and Surface-Water Elevations in the Fairbanks International Airport Area, Alaska, 1990-94
}

\author{
By David V. Claar and Michael R. Lilly
}

\begin{abstract}
Ground-water and surface-water elevation data were collected at 52 sites from 1990 to 1994 by the U.S. Geological Survey in cooperation with the Alaska Department of Transportation and Public Facilities, Fairbanks International Airport. Water elevations were measured in 32 groundwater observation wells and at 20 surface-water sites to help characterize the geohydrology of the Fairbanks International Airport area. From 1990 to 1993, data were collected in the vicinity of the former fire-training area at the airport. From 1993 to 1994, the data-collection area was expanded to include the entire airport area.
\end{abstract}

\section{INTRODUCTION}

The U.S. Geological Survey (USGS) in cooperation with the Alaska Department of Transportation and Public Facilities, Fairbanks International Airport (FIA), is presently (1995) conducting an investigation to characterize the geohydrology of the area around the FIA (fig. 1 and plate 1). The airport is located southwest of and adjacent to the city of Fairbanks. From 1990 to 1993, data were collected in the vicinity of the former fire-training area at the airport to characterize the geohydrologic conditions in this area. The current study is an expansion of work started in 1990 and includes the entire airport area. This report presents ground-water and surface-water-elevation data that were collected between October 1990 and October 1994.

Water elevations at many of the ground-water and surface-water sites were collected at monthly intervals. In addition, water-elevation data at selected sites were collected at more frequent intervals to document short-term changes in ground-water elevations caused by rapid stage changes of the Chena and Tanana Rivers. All ground-water data are maintained in the USGS Ground-Water Site Inventory (GWSI) data base. Surface-water data are in the USGS Automatic Data Acquisition and Processing System (ADAPS) data base (table 1).

\section{HYDROLOGIC SETTING}

The FIA study area occupies about $8 \mathrm{mi}^{2}$ of the flood plain between the Chena and Tanana Rivers at their confluence (plate 1). The former FIA fire-training area encompasses about $0.1 \mathrm{mi}^{2}$ near the southwest corner of the FIA (plates 1 and 2). The subsurface material at the study area and most of the surrounding area is composed of alluvial sand and gravel, known as the Chena Alluvium, deposited by the Tanana River (Péwé and others, 1976). Originally, the Chena River entered the Tanana River about $7 \mathrm{mi}$ east of Fairbanks. In 1940, the U.S. Army Corps of Engineers blocked several sloughs between the Chena and Tanana Rivers, redefining the mouth of the Chena River to a location 7 mi southwest of Fairbanks (T.L. Péwé, written commun., 1995). Sediment facies within the deposits are laterally discontinuous as is typical of braided river deposits (Rust, 


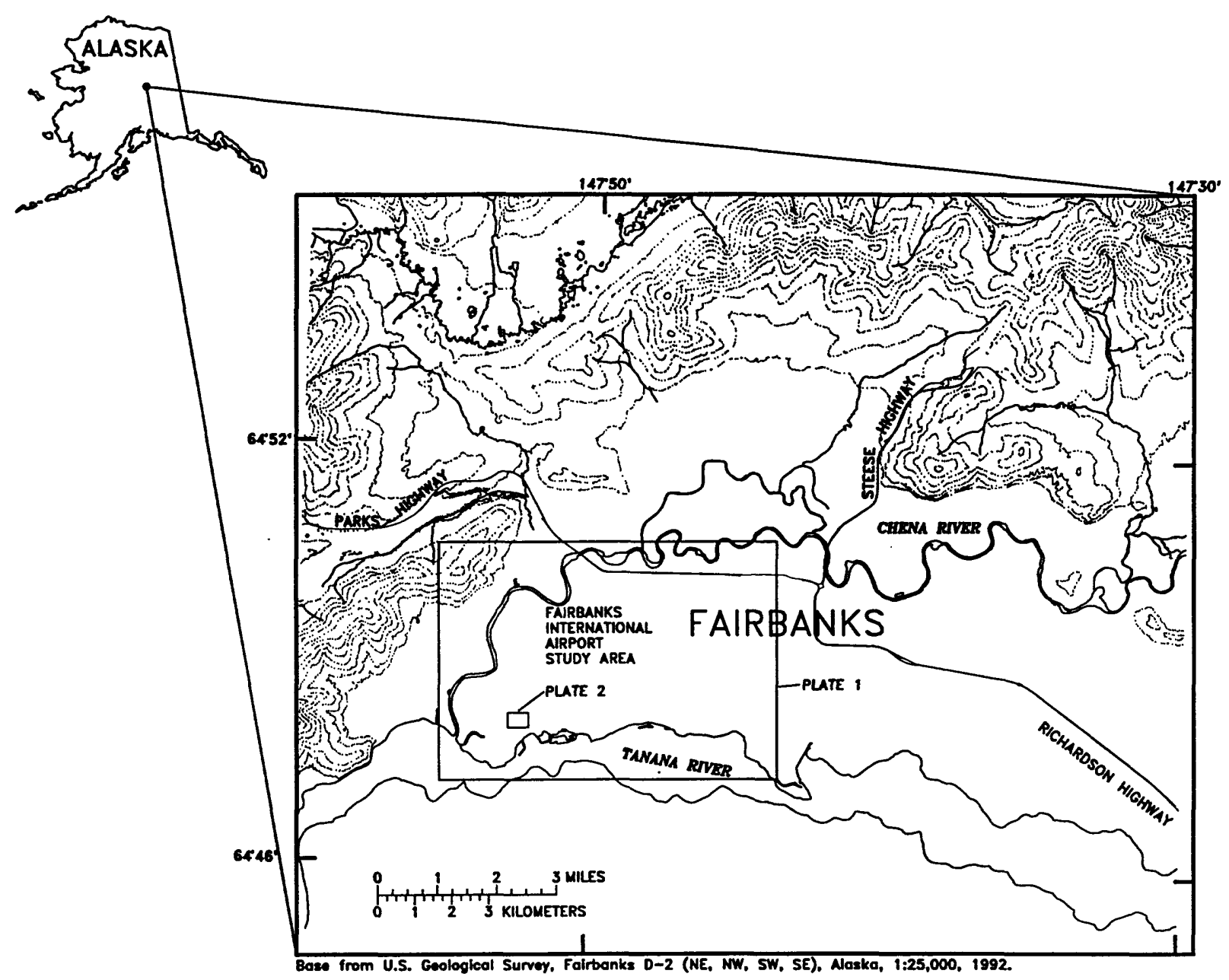

Figure 1. Location of Fairbanks, Alaska, and the Fairbanks International Airport study area.

1978). The thickness of these deposits is unknown but may be in excess of $500 \mathrm{ft}$ (Nelson, 1978). Chena Ridge, which is located north of the Chena River along the northwest boundary of the study area, is part of a metamorphic system that forms the Yukon-Tanana Upland (Anderson, 1970).

The Tanana River is the main surface-water influence on much of the alluvial aquifer in the general Fairbanks area (Nelson, 1978). The Tanana River stage typically rises for one to two weeks during spring snowmelt and from ice-jam effects and then recedes (fig. 2). The stage again rises for a longer period during the middle of the summer in response to glacial runoff from the Alaska Range. The flow and stage of the Tanana River decrease during late summer when temperatures drop in the Alaska Range. The river stages increase after complete ice cover is established across the river as a result of an increase in flow resistance from ice cover. Stages then decrease throughout the winter because of the continuing reduction of water flow.

Stages in the reach of the Chena River within the study area are affected by backwater from the Tanana River, as well as by variation in flow in the Chena River. Flows in the Chena River rise during spring snowmelt runoff and late summer rainfall runoff (fig. 2). Ice also covers most of the Chena River during the winter season, although long reaches may remain ice free throughout the winter. 


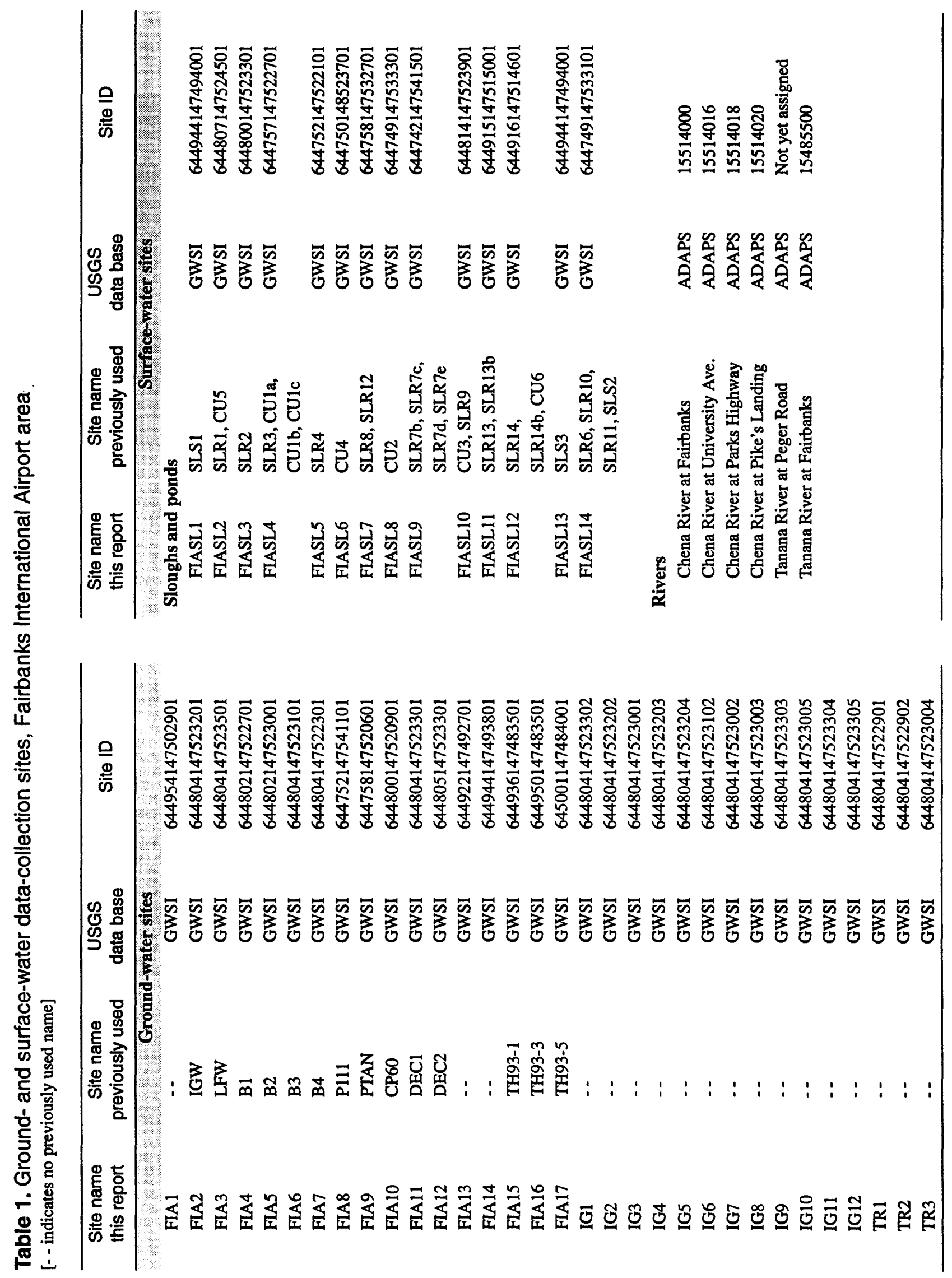



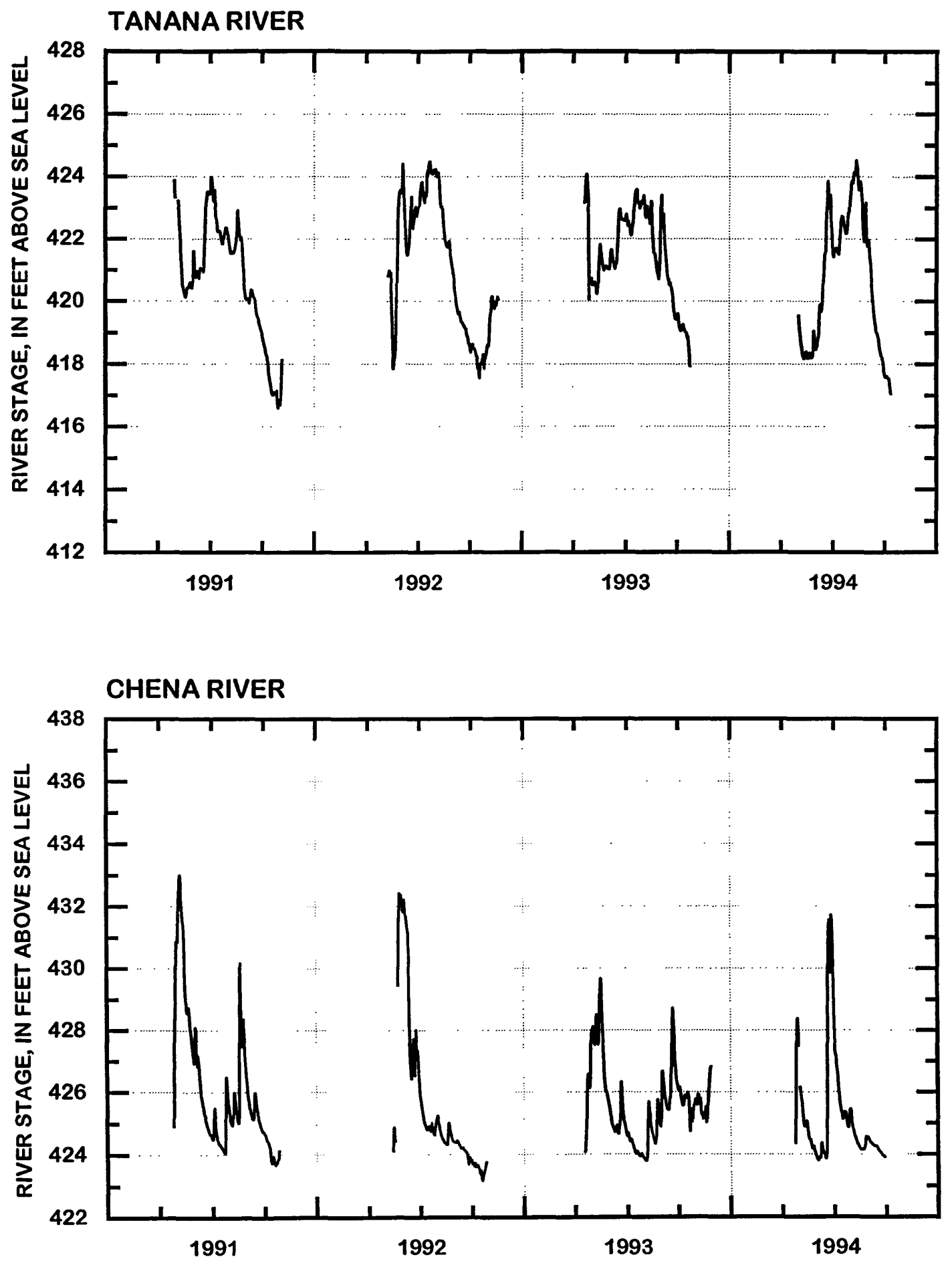

Figure 2. Water-surface elevations of the Tanana and Chena Rivers at Fairbanks gaging stations for calendar years 1991 to 1994 . 
The depth to ground water below the land surface is less than $10 \mathrm{ft}$ throughout most of the study area. Ground-water elevations respond to changes in stage of the Chena and Tanana Rivers. No major withdrawals of ground water within the study area affect regional ground-water levels. However, water levels within the former fire-training area were affected by small-scale pumping during short periods of time when ground-water remediation experiments were conducted during the summer of 1992. The College Utilities Corporation also withdraws water from wells on the north side of the Chena River close to the northeast corner of the study area. The effect of these withdrawals on water levels within the study area is unknown, but is thought to be insignificant over most of the study area.

\section{GROUND-WATER SITES}

During this study, water elevations were measured in 32 wells, 23 of which are in the former fire-training area (plates 1 and 2, and table 1). All wells are shallow-less than $30 \mathrm{ft}$ deep-and most are screened at the water table. Data from some of the wells have been previously reported or released to the public using well names that are different from those used in this report. Present and previous names are cross referenced in table 1.

The water elevation in a well was determined by using either a steel tape and chalk or an electric tape to measure the vertical distance between a measuring point and the water surface. The electric tapes were calibrated against steel tapes and correction factors were applied. Replicate measurements were taken at each well until depth-to-water readings were duplicated within $0.01 \mathrm{ft}$ for measurements made by steel tapes and within $0.02 \mathrm{ft}$ for those made by electric tapes. The measuring points at all wells used in this study were labeled to avoid errors.

\section{SURFACE-WATER SITES}

Surface-water elevations were measured at 20 sites: 4 on the Chena River, 2 on the Tanana River, and 14 on miscellaneous sloughs and ponds (plates 1, 2 and table 1). The Chena River at Fairbanks gaging station (not shown on plate 1) is located about $0.5 \mathrm{mi}$ downstream from the Steese Highway bridge. The sloughs and ponds act as retention ponds for snowmelt and rainfall runoff. Water elevations in sloughs, ponds, and selected river sites were determined by reading a staff gage or by using a measuring tape to measure the distance from the water surface to a point of known elevation. At stream-gaging stations, water elevations were determined by using wireweight gages on bridges, by surveying to the water surface, or by water-level sensors interfaced with a continuous stage recorder. In the winter, holes were cut through the ice to measure water elevations.

\section{SURVEY DATA}

Elevation of reference points at all water-elevation measuring sites in this study were surveyed to the National Geodetic Vertical Datum of 1929. The datum is called "sea level" in this report. The reference benchmark located on the main airport runway is K 60 (U.S. Coast and Geodetic Survey, 1966). The elevation of K 60 used for this study and used by the Fairbanks International Airport is $432.95 \mathrm{ft}$. This elevation is $0.02 \mathrm{ft}$ higher than the elevation given by the U.S. Coast and Geodetic Survey (1966). Before June 1992, the USGS used a different benchmark; pre- 
viously released elevations may differ from those given in this report. The elevations of reference points forming the FIA vertical survey net were connected to the vertical survey net used by the USGS in a study of the University of Alaska area, located northeast of the FIA.

Selected wells were re-surveyed to check and adjust elevations for frost jacking. Frost jacking occurs mainly in late spring or early summer, after the snow has melted. Updated measuring point elevations are applied to water elevations after the estimated time of movement. Horizontal locations of sites were determined by horizontal ground surveys, satellite-based global positioning systems, and from maps of the study area.

\section{GROUND-WATER AND SURFACE-WATER ELEVATIONS}

The remainder of this report consists of a section called "Water-Elevation Tables and Hydrographs." This section shows ground-water and surface-water data collected in the Fairbanks International Airport area between 1990 and 1994. Data for each site consist of water elevations collected (1) during mass measurements (MM) - sets of measurements made once each month at a large group of sites within a short time period, and (2) during partial measurements (PM) - sets of measurements made as needed at a smaller selection of sites.

Tables: Each table showing water elevations contains information about the site, well construction, and each measurement made at the site. Land surface (LS) is the average elevation of the ground immediately surrounding the well, rebar, culvert, or staff gage at each site. The measuring point (MP) is the point of known elevation from which all water-elevation measurements are taken. The site identification (ID) is based on latitude and longitude and is the primary identifier used in the USGS data bases GWSI and ADAPS. The local number is based on the State Rectangular Coordinate System, defined by base and meridian, township, range, section, and quarter section to the fourth order number (fig. 3).

Hydrographs: Each hydrograph shows all data presented on the corresponding water-elevation table. Each point on the hydrograph represents a measured water elevation. Uniform time and elevation scales for each hydrograph are used to allow direct comparison between hydrographs. Data points are plotted and connected with lines. These lines show the estimated trends between measurements; however, the connecting lines do not reflect any shorter period stage changes that may have occurred between measurements. No trend lines were drawn between points 2 months or more apart.

\section{REFERENCES CITED}

Anderson, G.S., 1970, Hydrologic reconnaissance of the Tanana Basin, Central Alaska: U.S. Geological Survey Hydrologic Investigations Atlas HA-319, 4 sheets.

Nelson, G.L., 1978, Hydrologic information for land-use planning, Fairbanks vicinity, Alaska: U.S. Geological Survey Open-File Report 78-959, 47 p.

Péwé, T.L., Bell, J.W., Forbes, R.B., and Weber, F.R., 1976, Geologic map of the Fairbanks D-2 SW quadrangle, Alaska: U.S. Geological Survey Miscellaneous Investigations Series Map I-829-A, scale, 1:24000, 1 sheet.

Rust, B.R., 1978, Depositional models for braided alluvium, in Miall, A.D., ed., Fluvial sedimentology: Canadian Society of Petroleum Geology Memoir 5, p. 605 - 625.

U.S. Coast and Geodetic Survey, 1966, Vertical control datum: U.S. Department of Commerce, Environmental Sciences Services Administration, September, 26 p. 


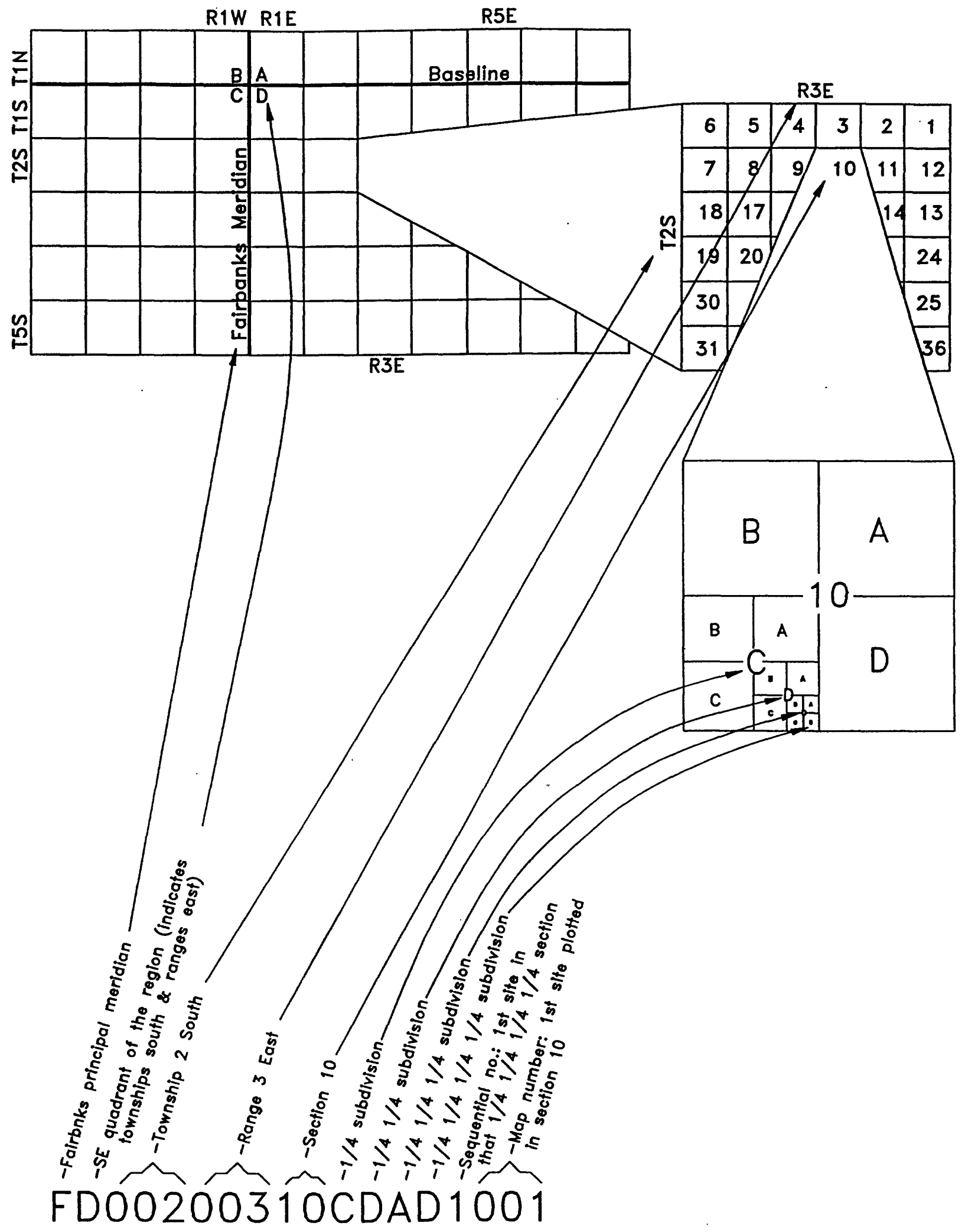

Figure 3. Derivation of local number from the official rectangular subdivision of public lands. 
WATER-ELEVATION TABLES AND HYDROGRAPHS 

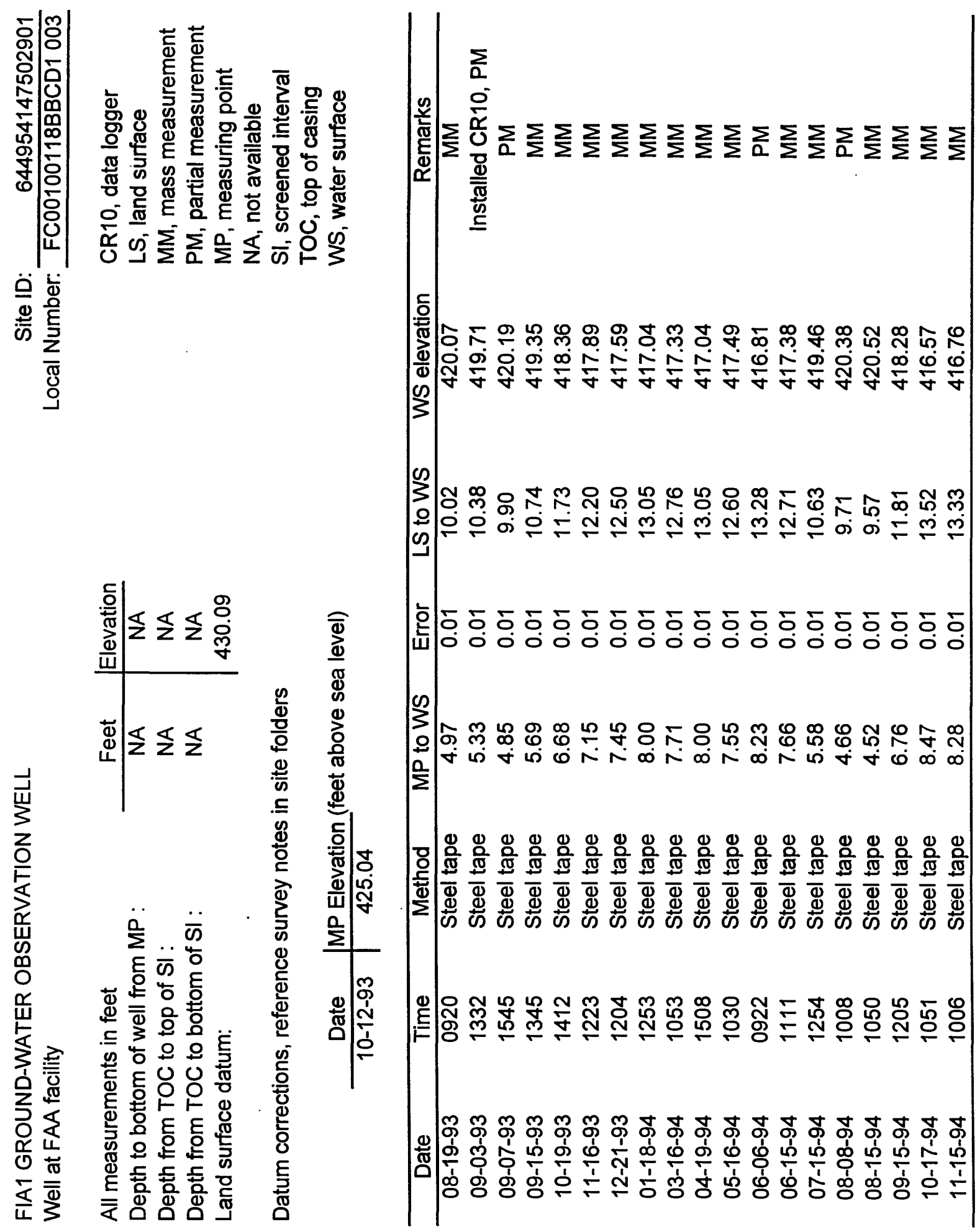


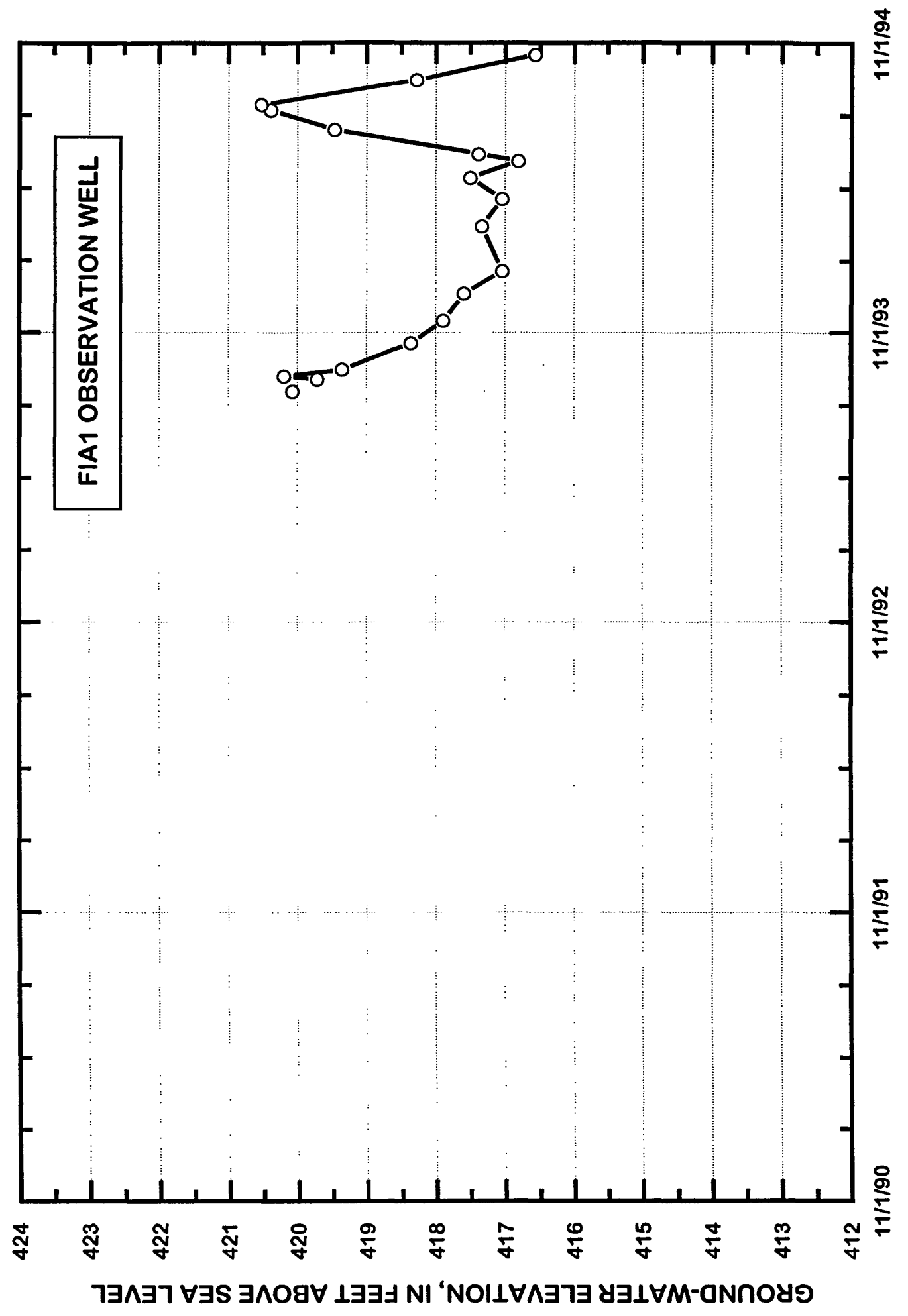



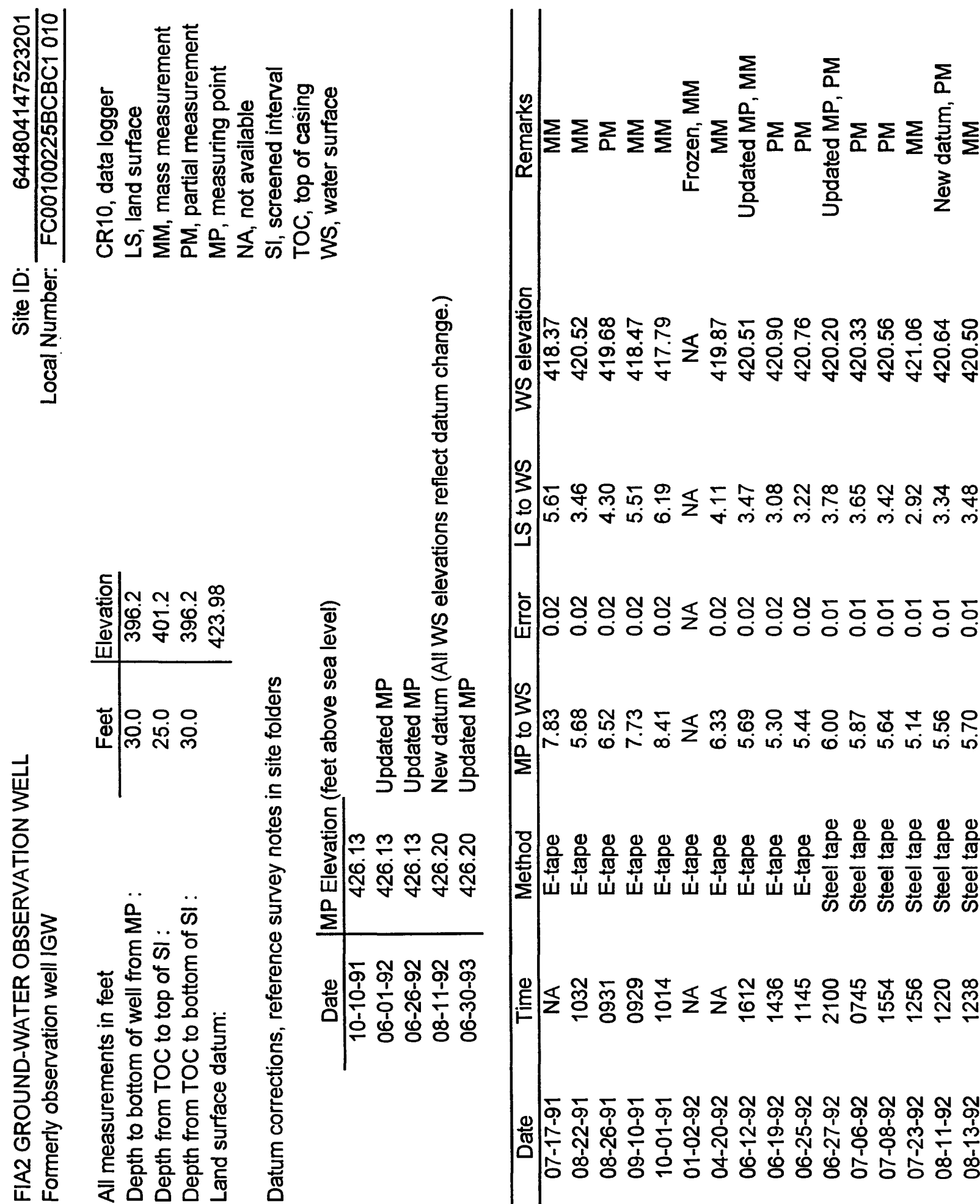

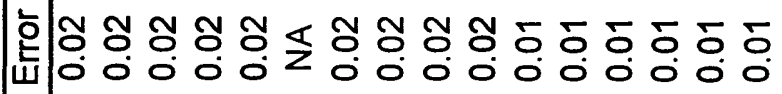

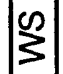

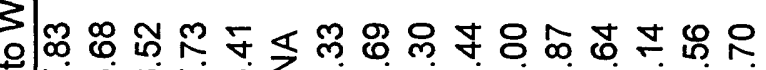

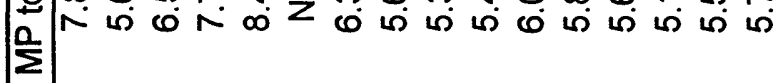

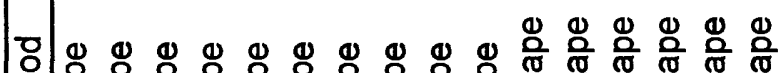
突雨

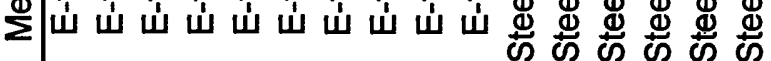

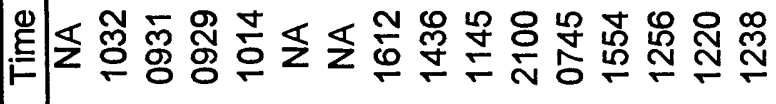

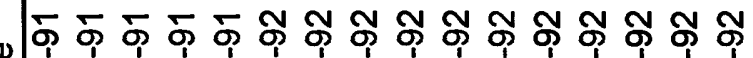

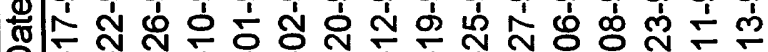

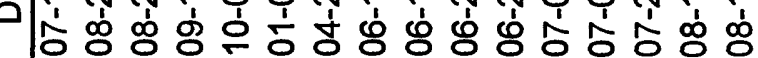




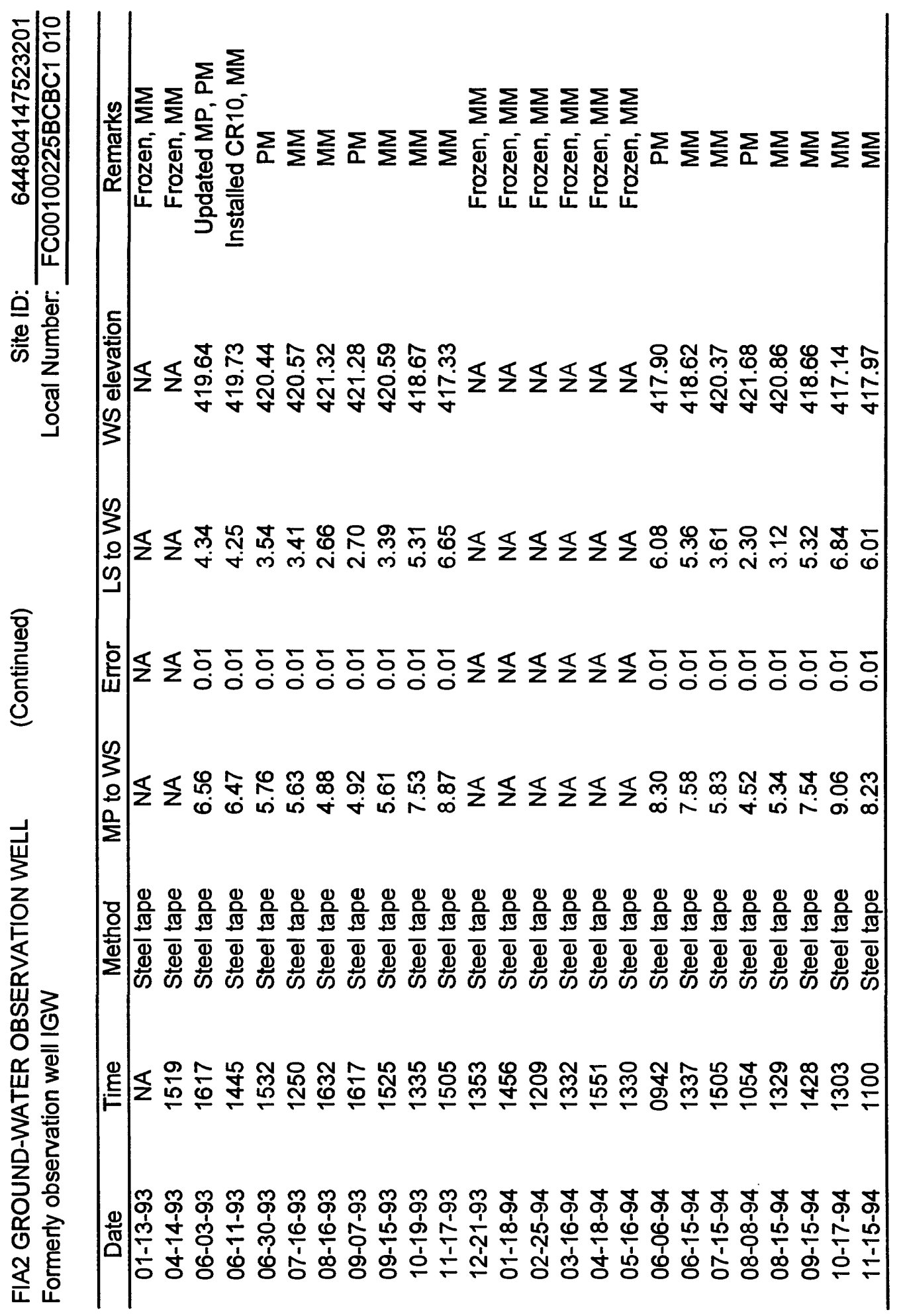


14 Ground-Water and Surface-Water Elevations in the Fairbanks International Airport Area, 1990-94 


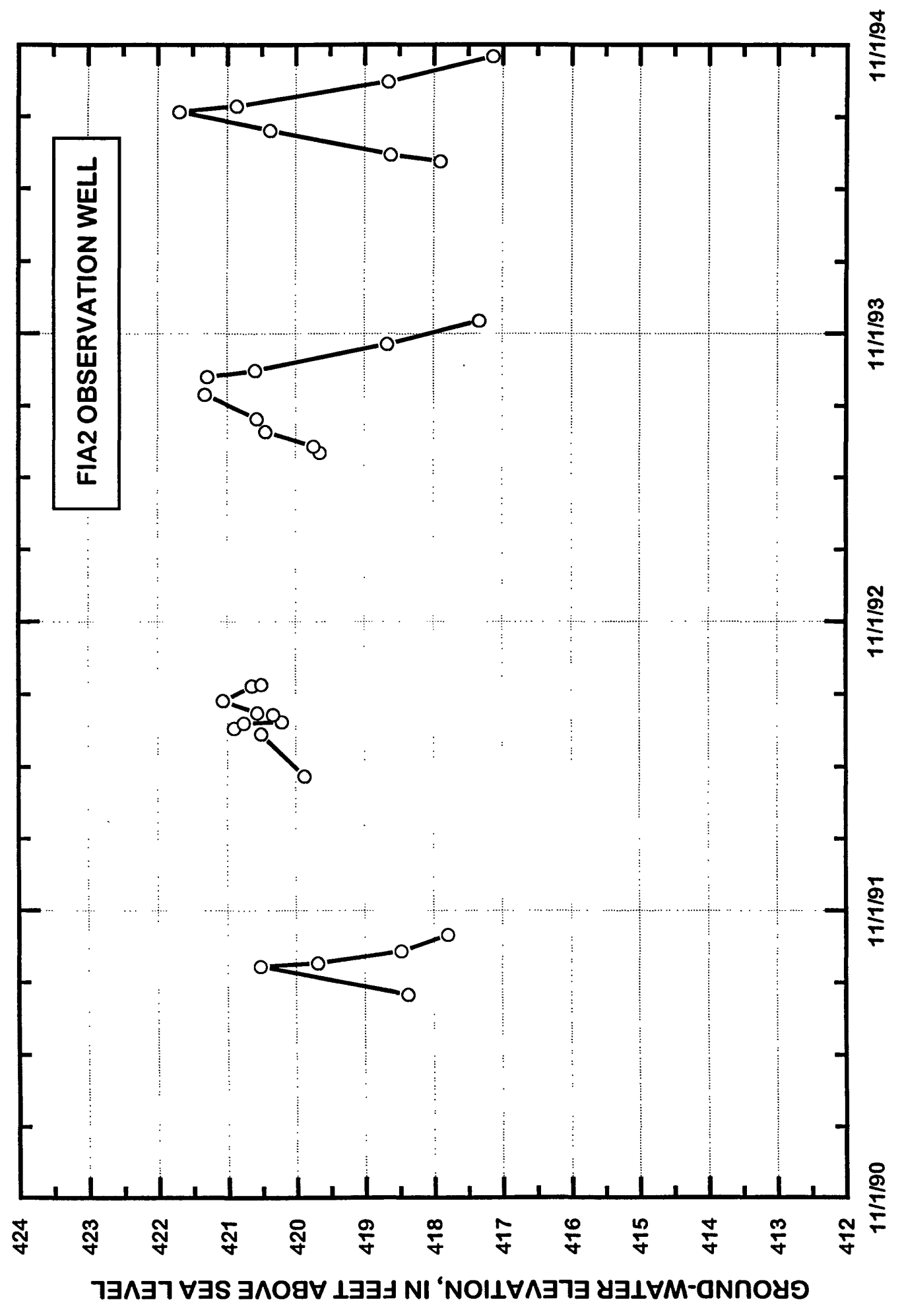



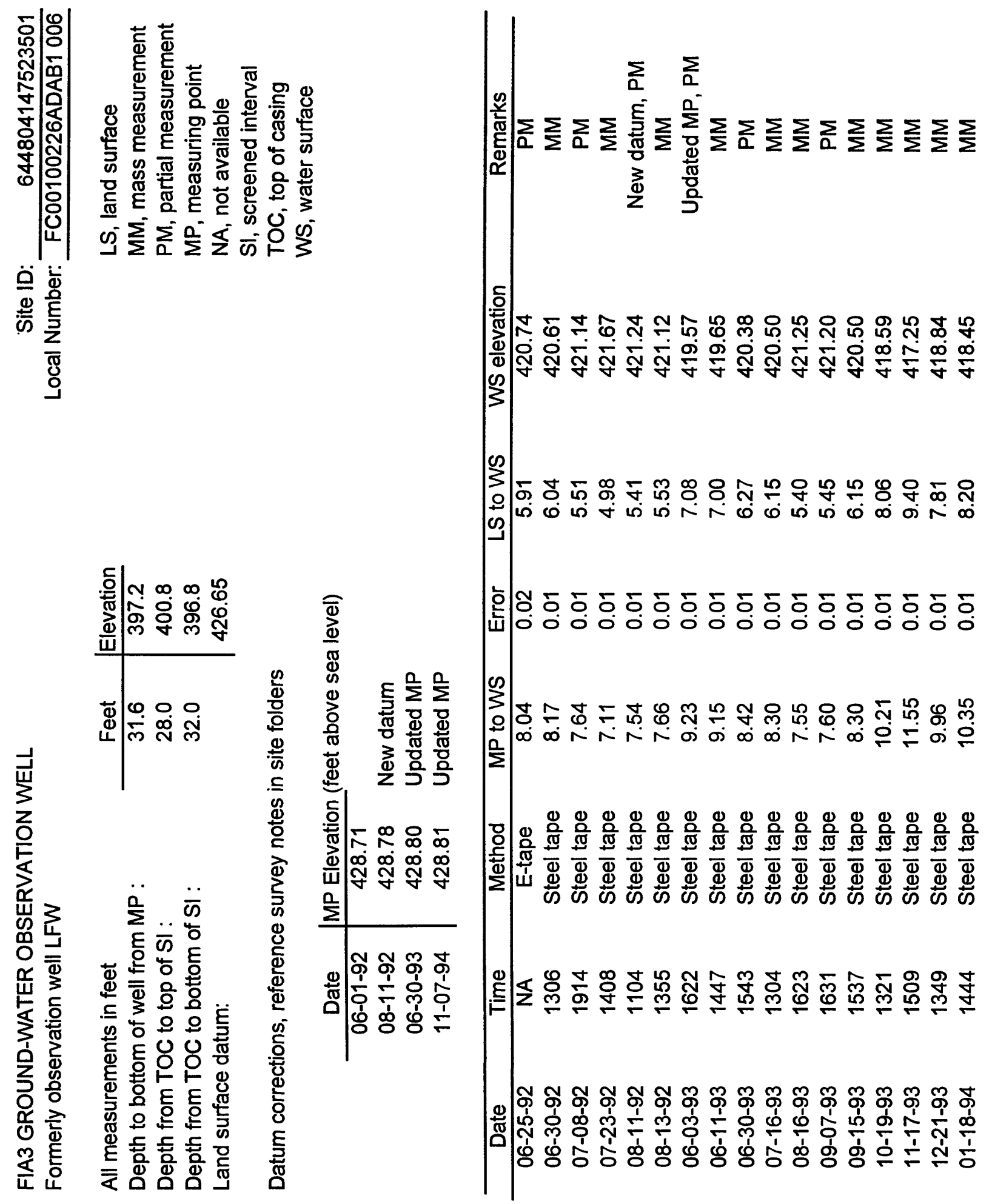


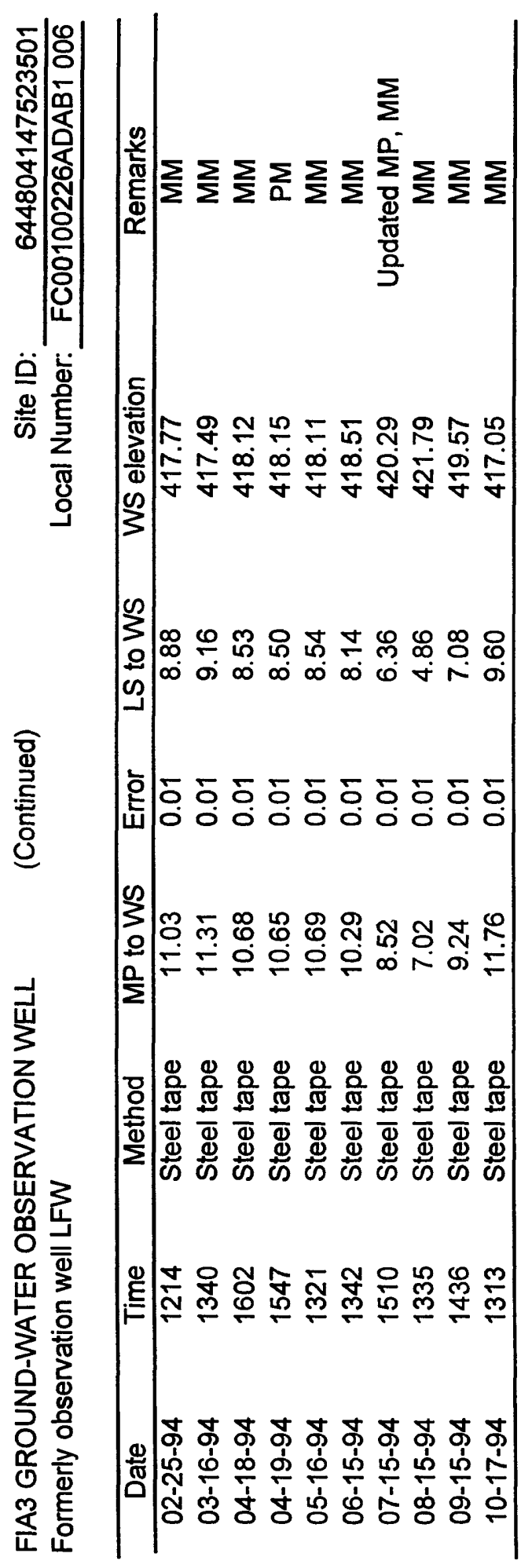


18 Ground-Water and Surface-Water Elevations in the Fairbanks International Airport Area, 1990-94 


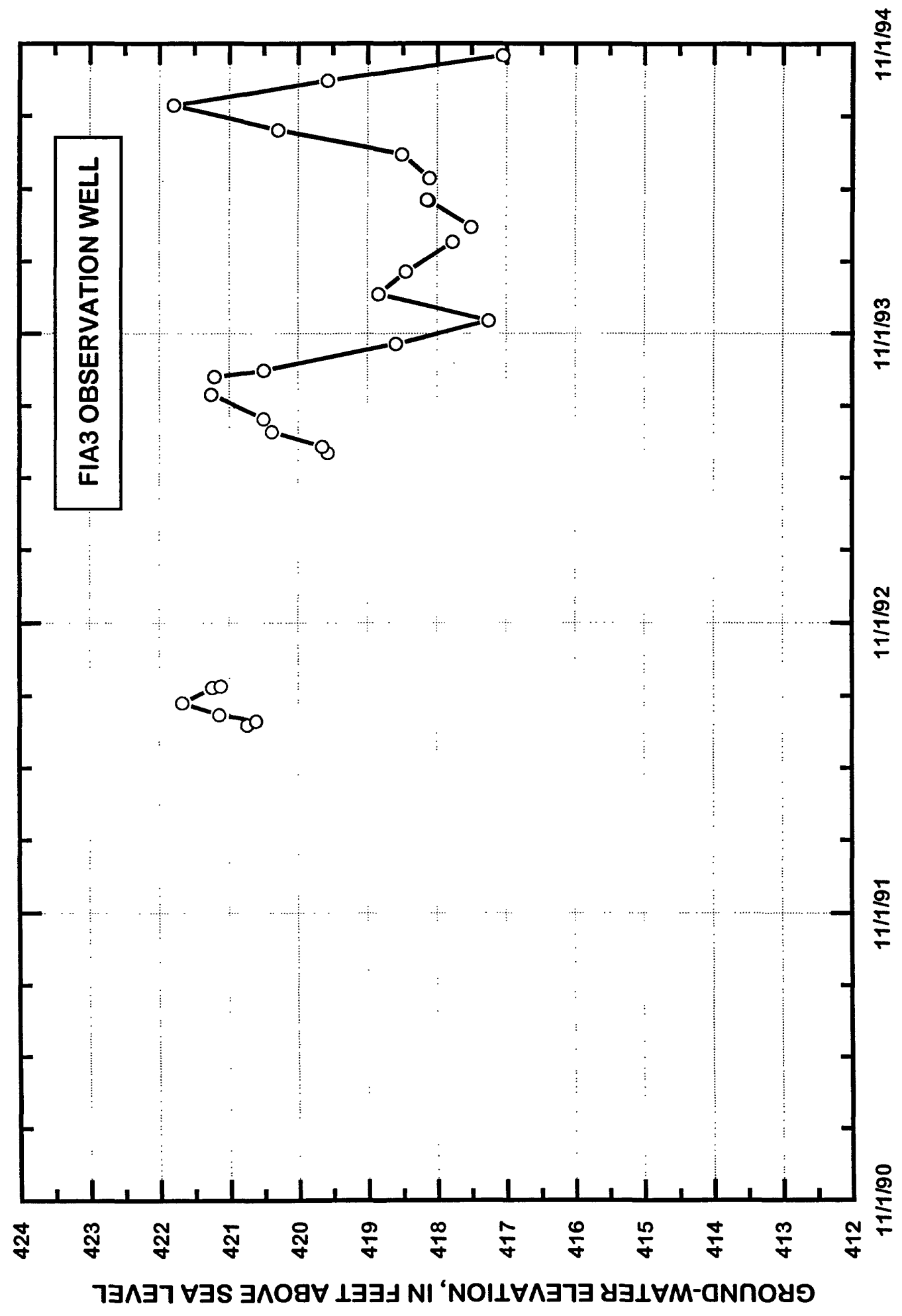

WATER-ELEVATION TABLES AND HYDROGRAPHS 19 

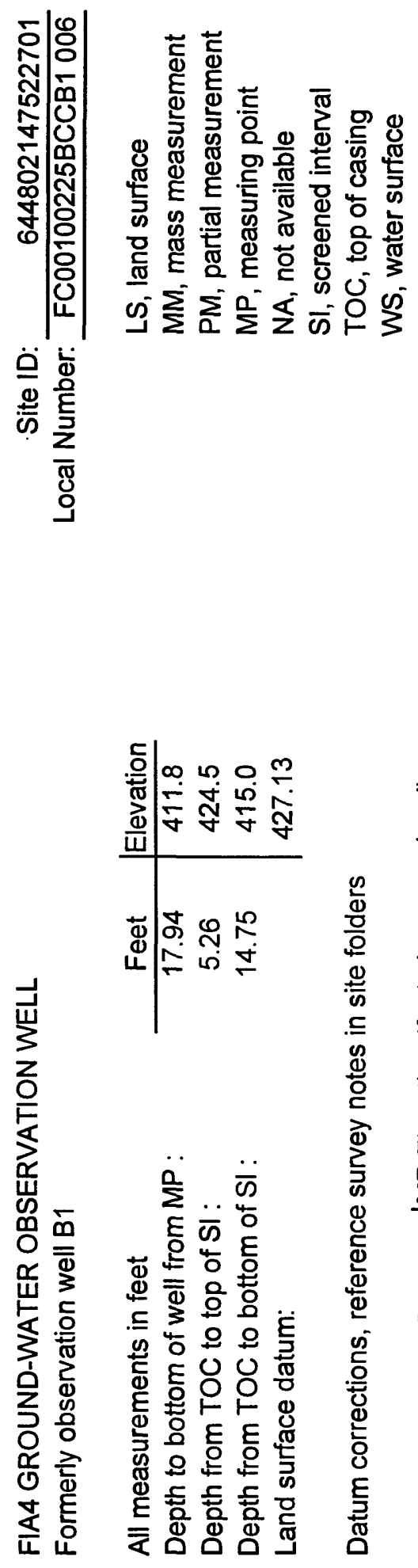

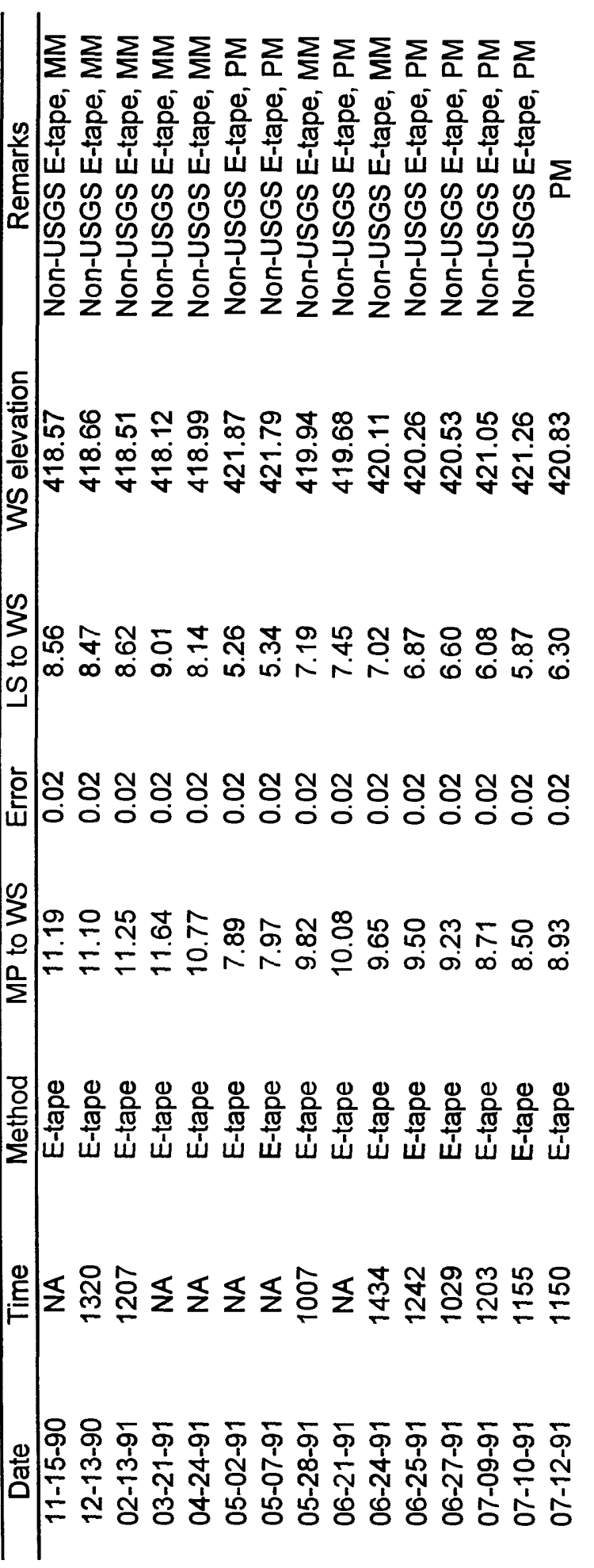




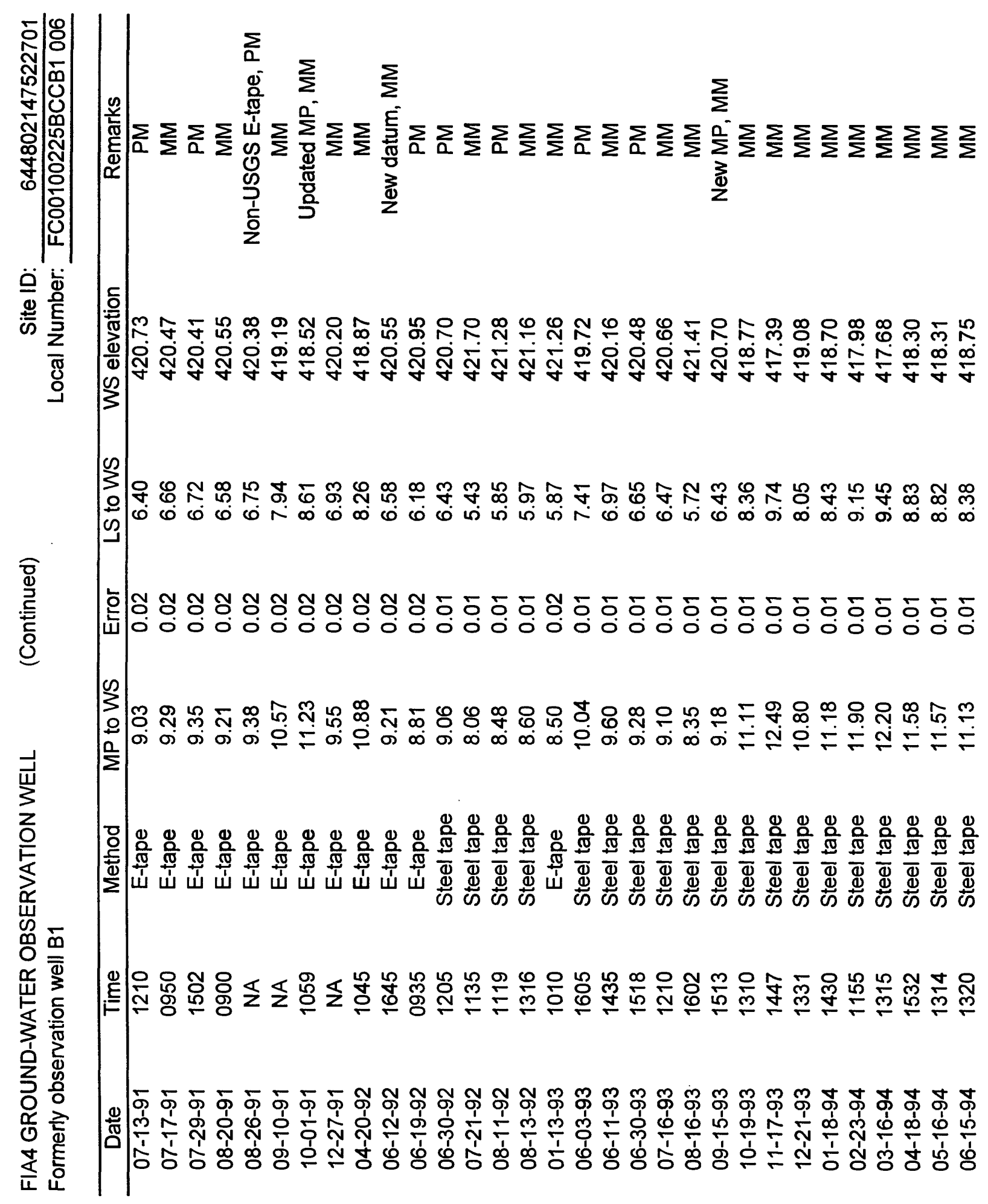




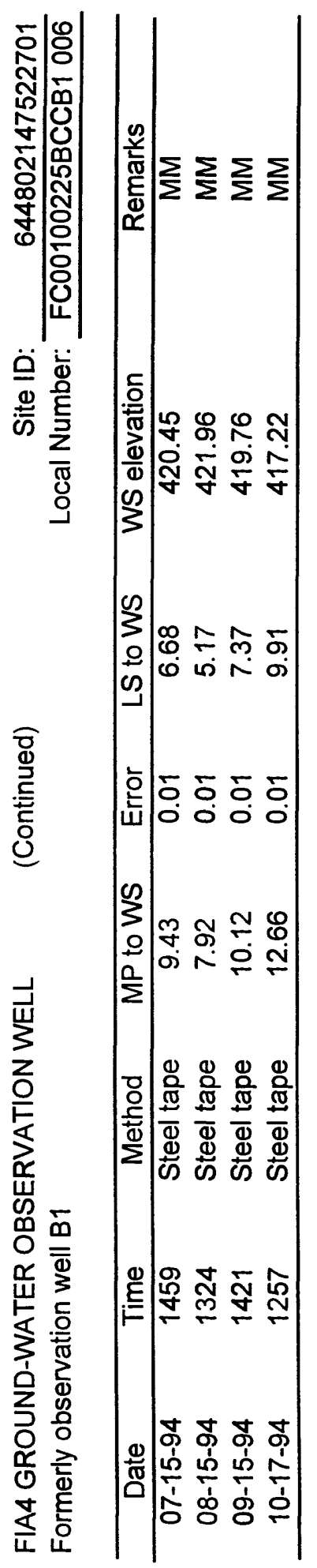




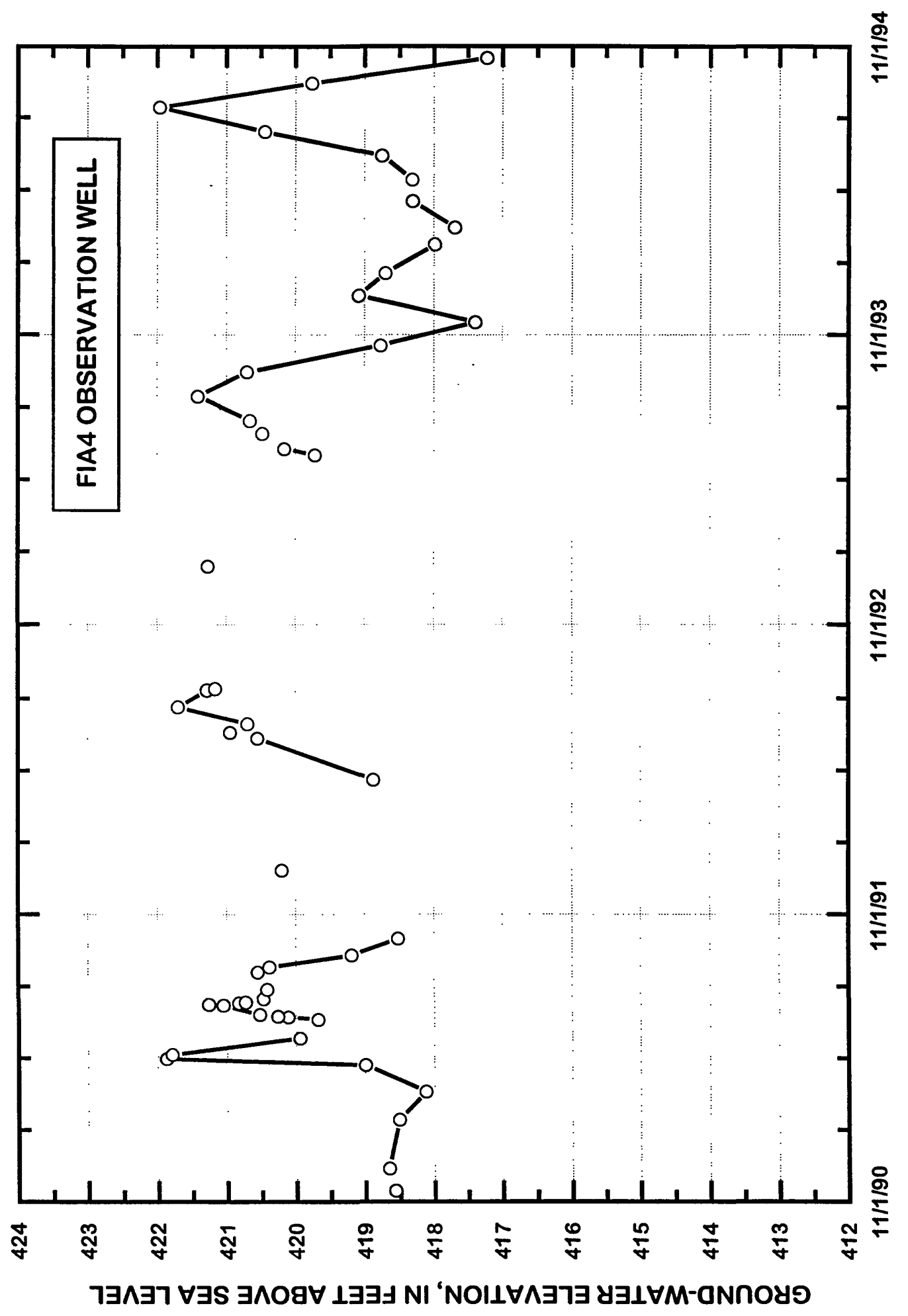




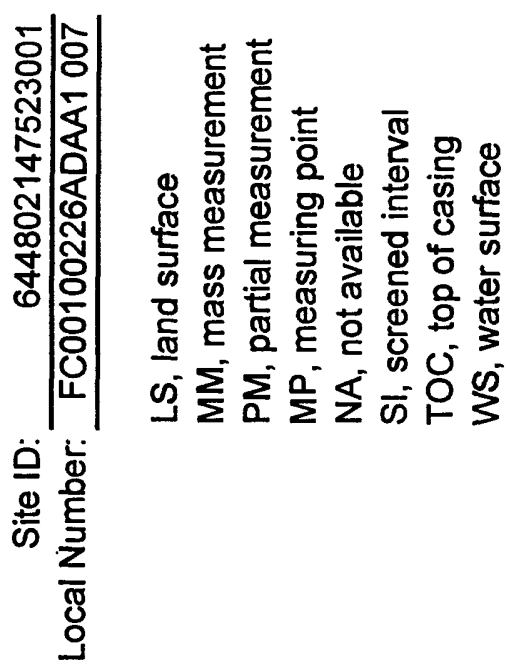

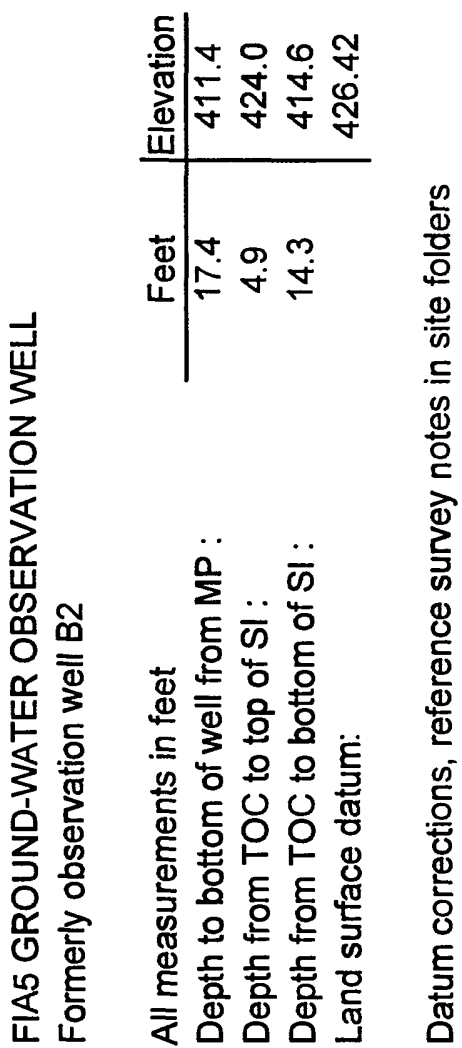

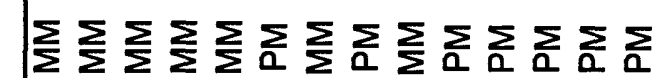

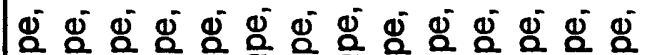

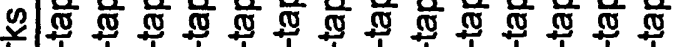
它

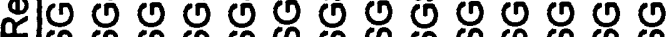
๘ ळ

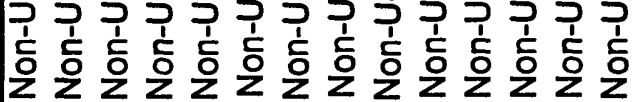

மீ

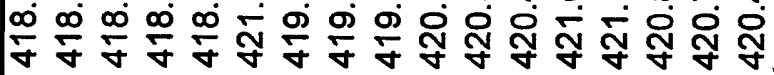

里

œ

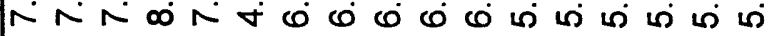

1

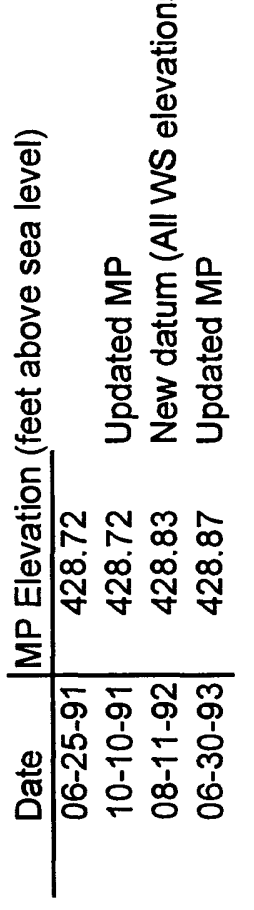

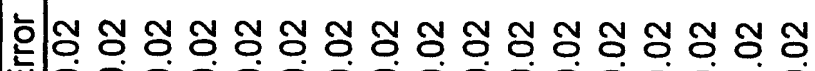

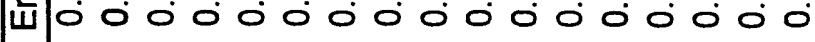

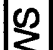

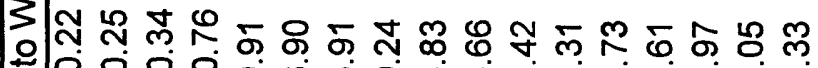
a 으응의 $\Sigma$

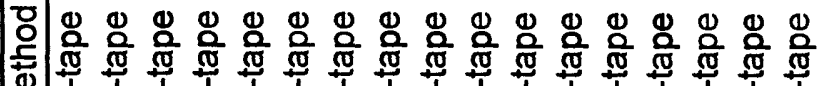

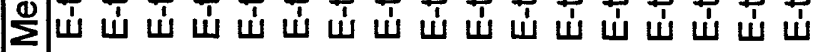

튼윤윤

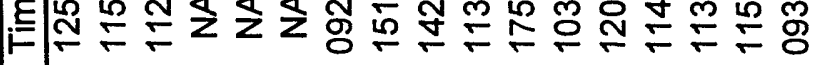

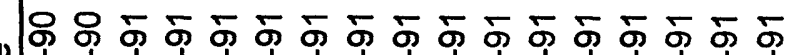
离 ดั 


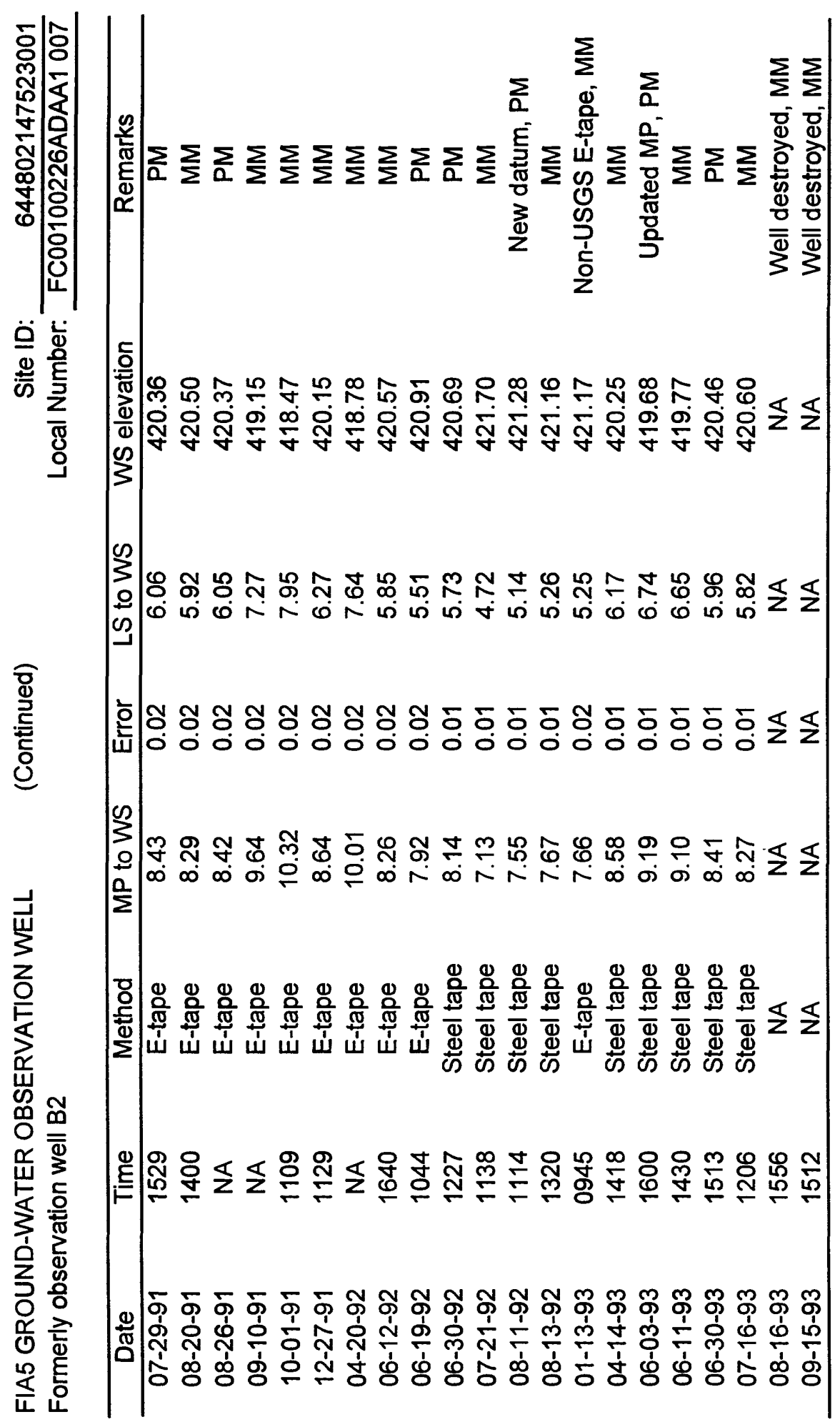


26 Ground-Water and Surface-Water Elevations in the Fairbanks International Airport Area, 1990-94 


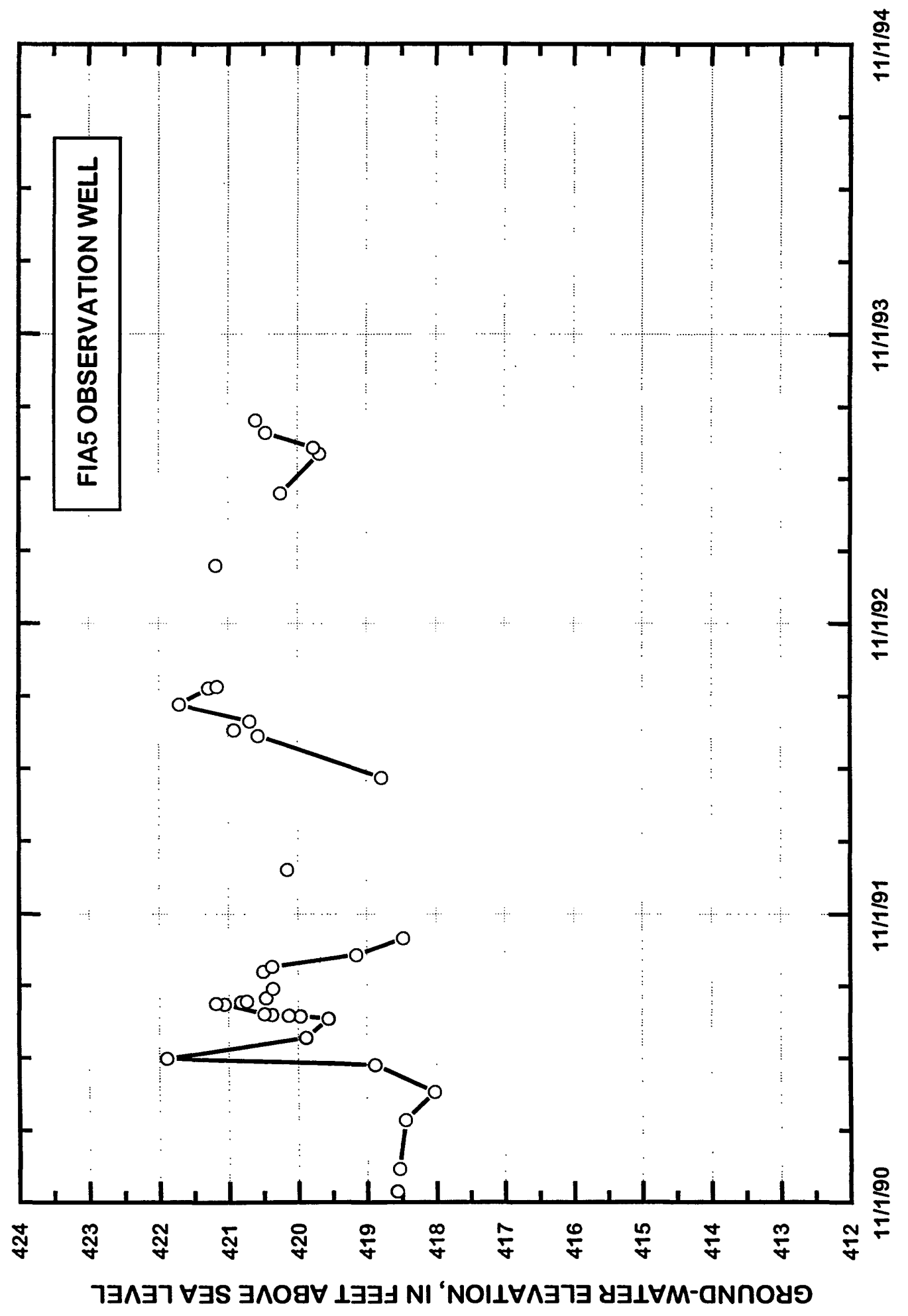



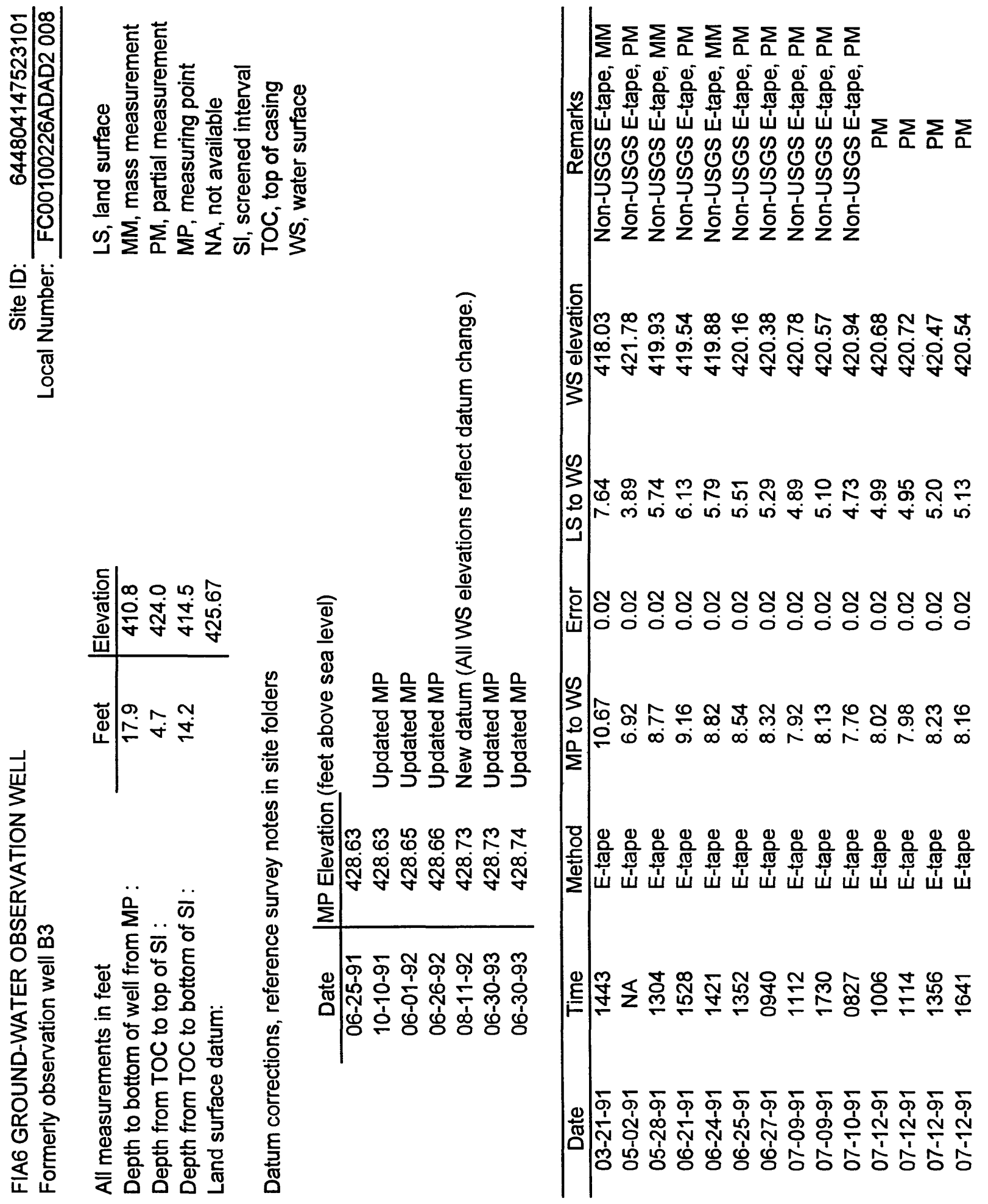

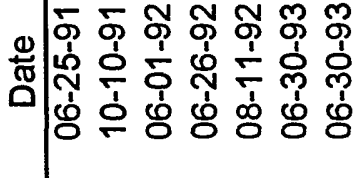

๘ூூ

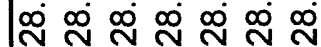

号

号员员空员员

유 융

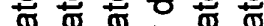

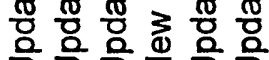

$\Phi$

윽음원음

\& $\forall$

它

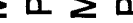

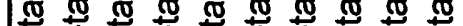

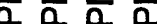

ळळ

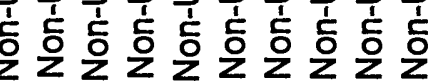

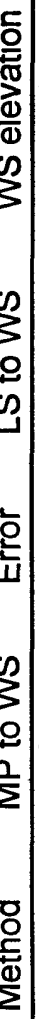

m 文 


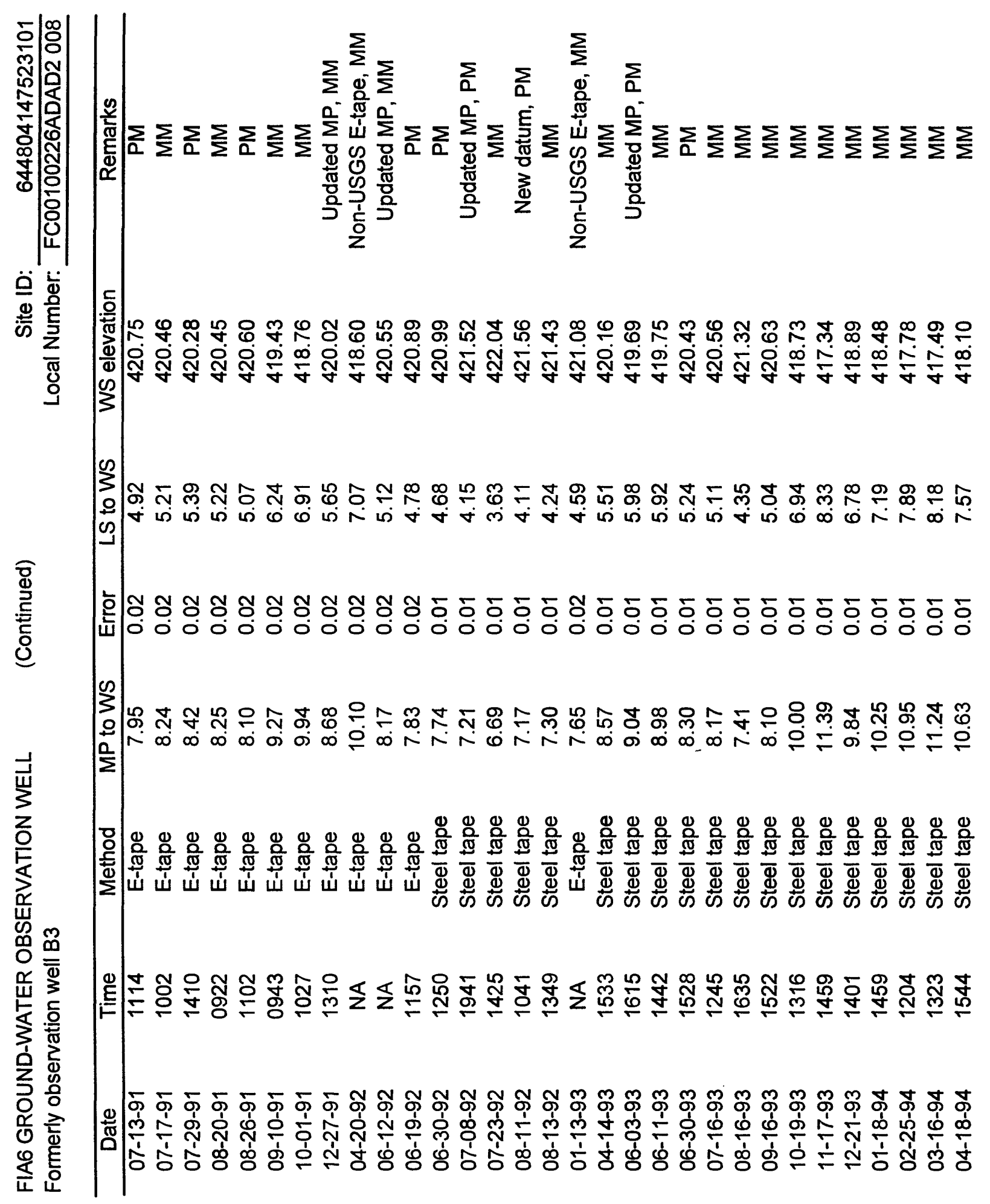




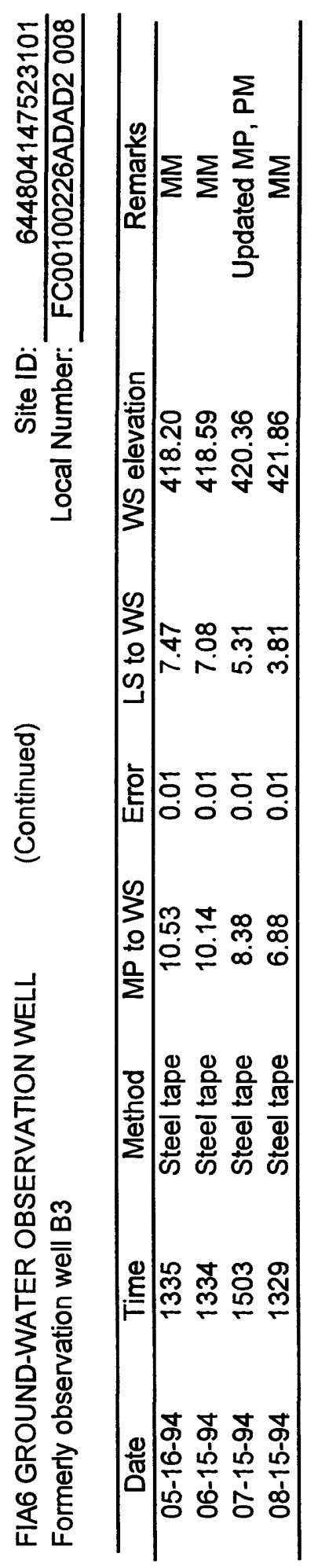




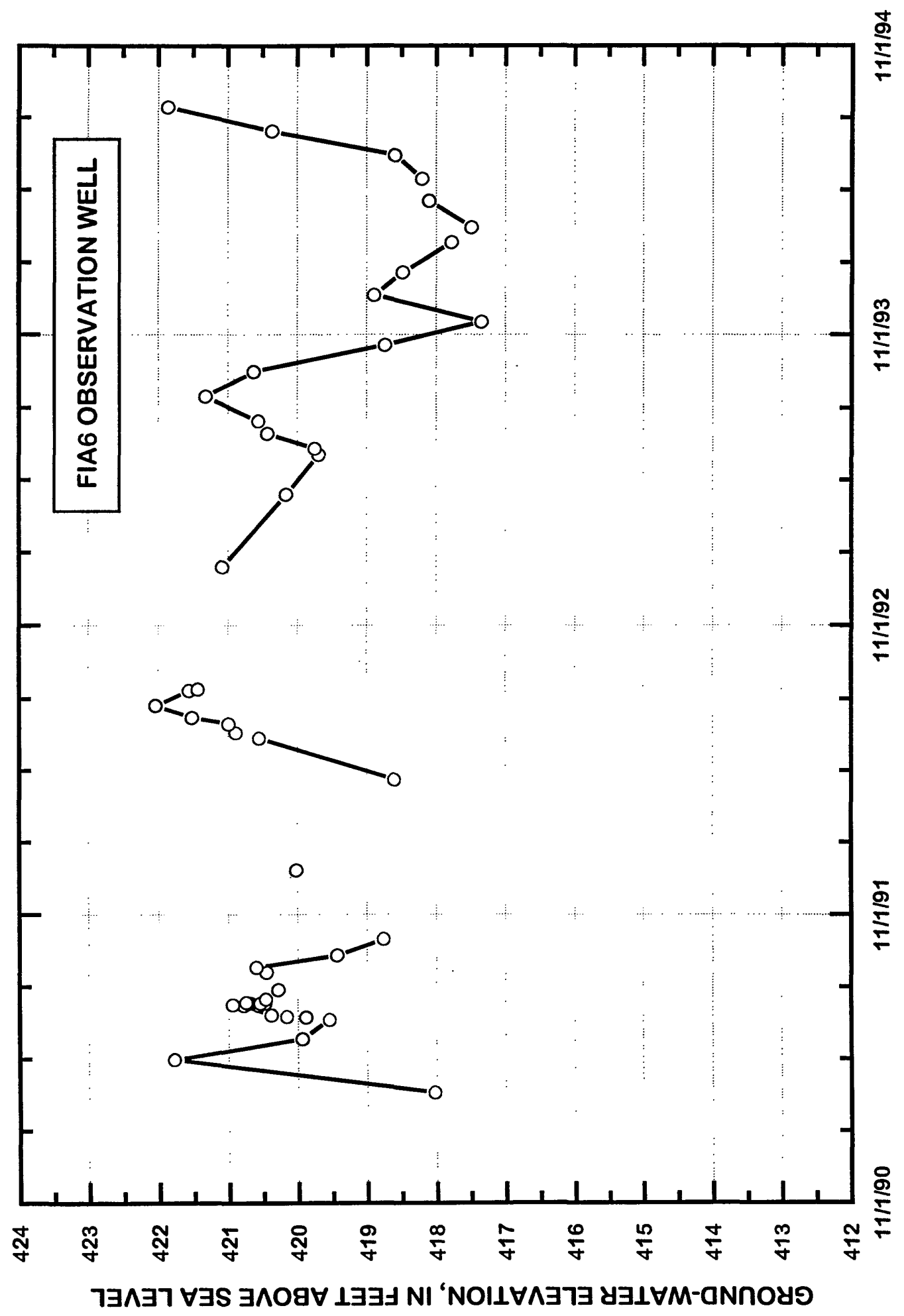




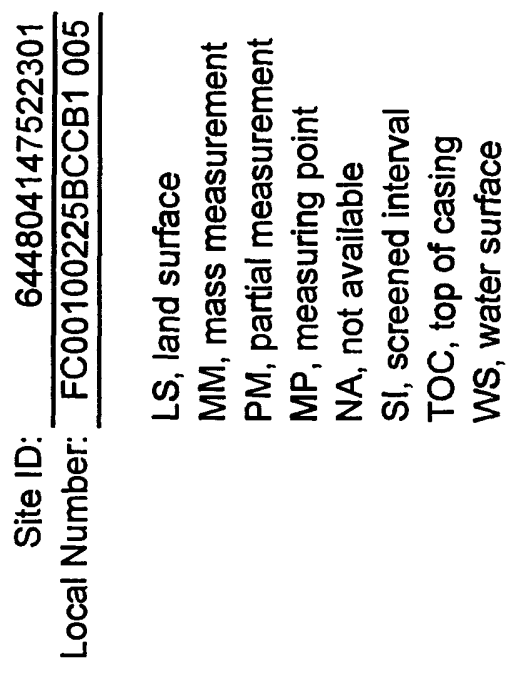

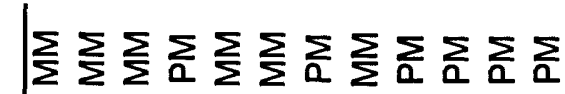

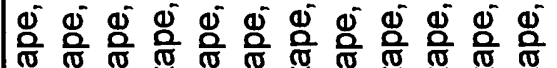
की ஜ

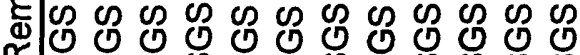

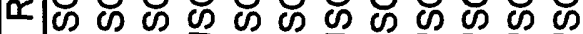

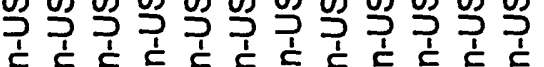

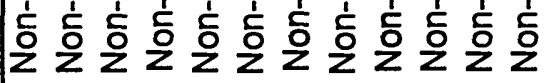

\section{등}

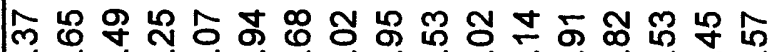

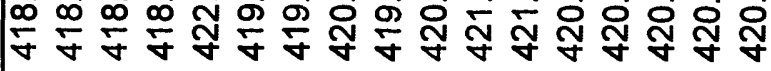

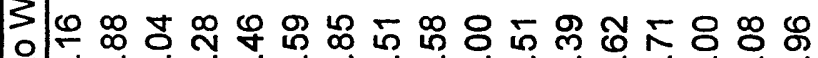
个 6 个

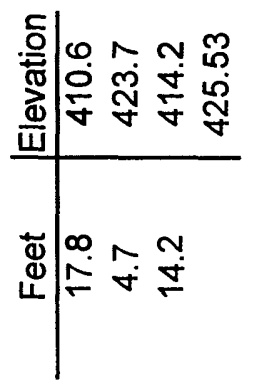

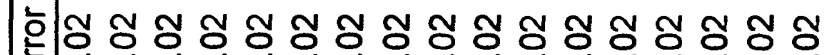

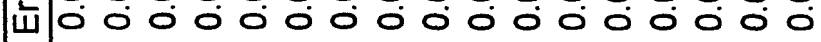

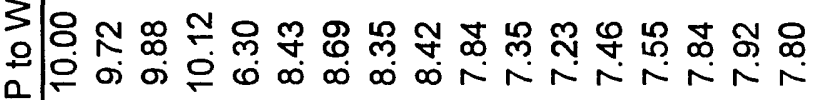
需 要

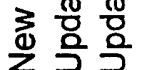
号

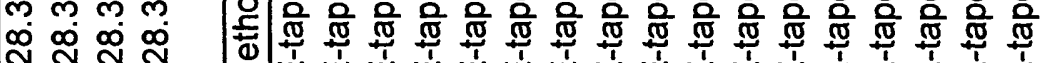
พั $\mathcal{Y}$ พ ய่ ธั ชิ ณั ชั బิ둥

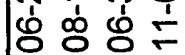

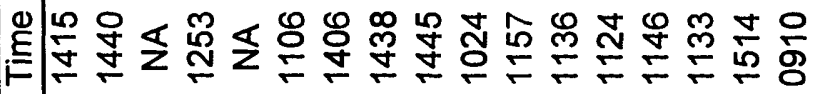

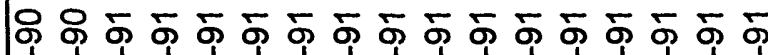

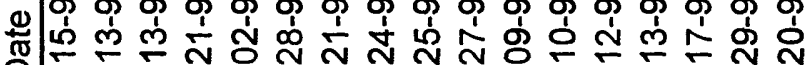

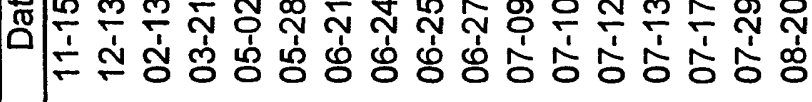




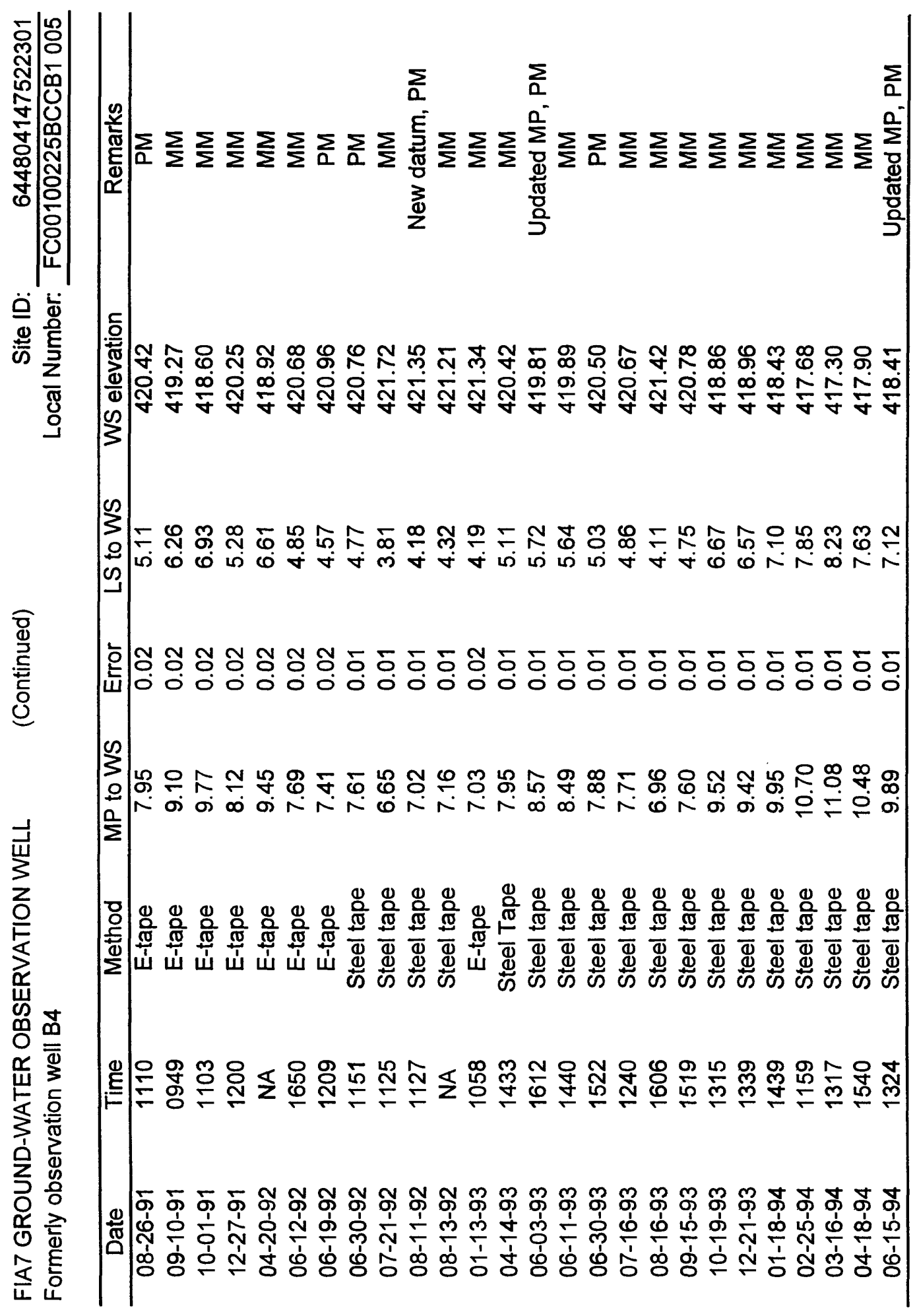


34 Ground-Water and Surface-Water Eievations In the Fairbanks internationai Alrport Area, 1990-94 


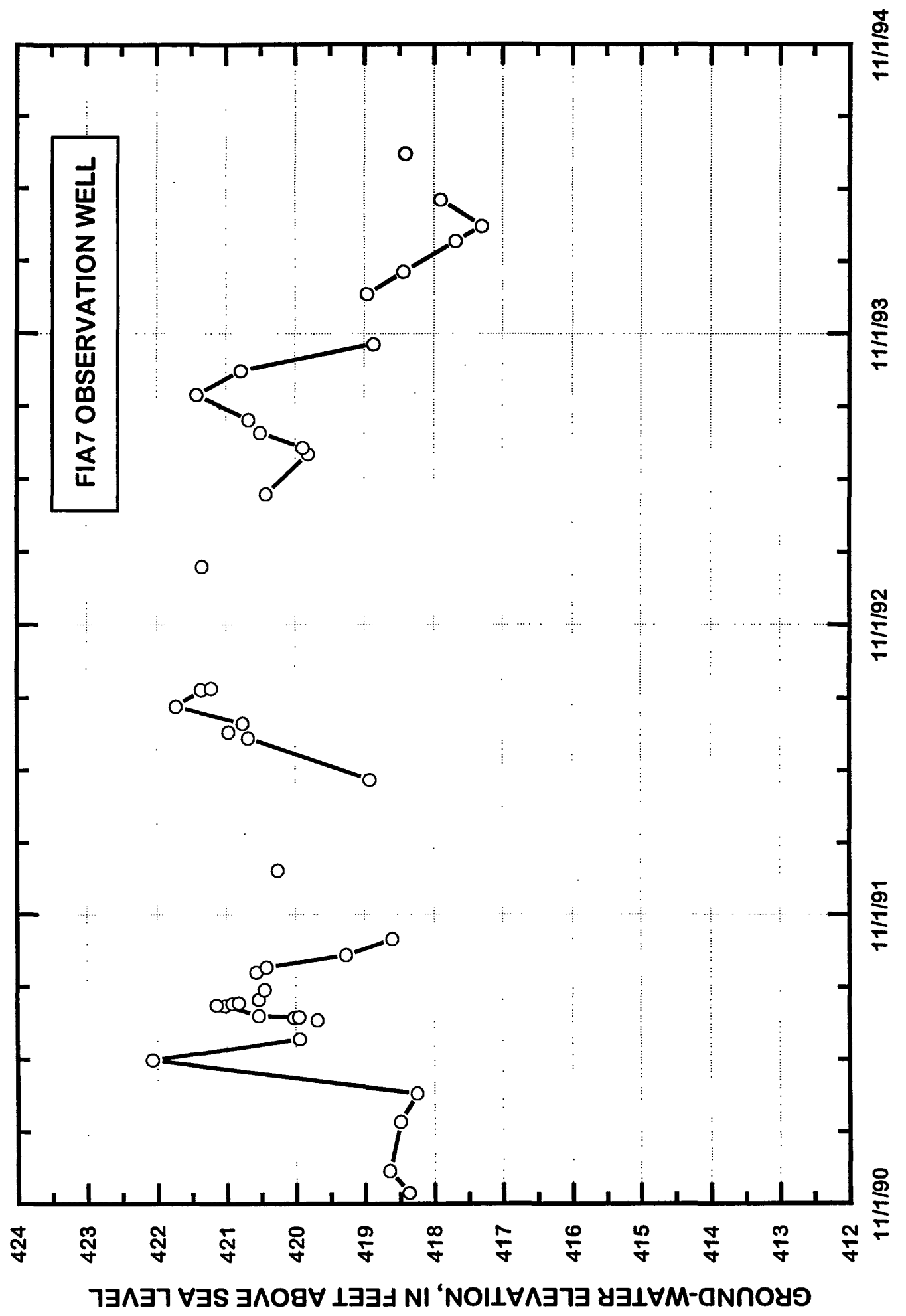

WATER-ELEVATION TABLES AND HYDROGRAPHS 35 

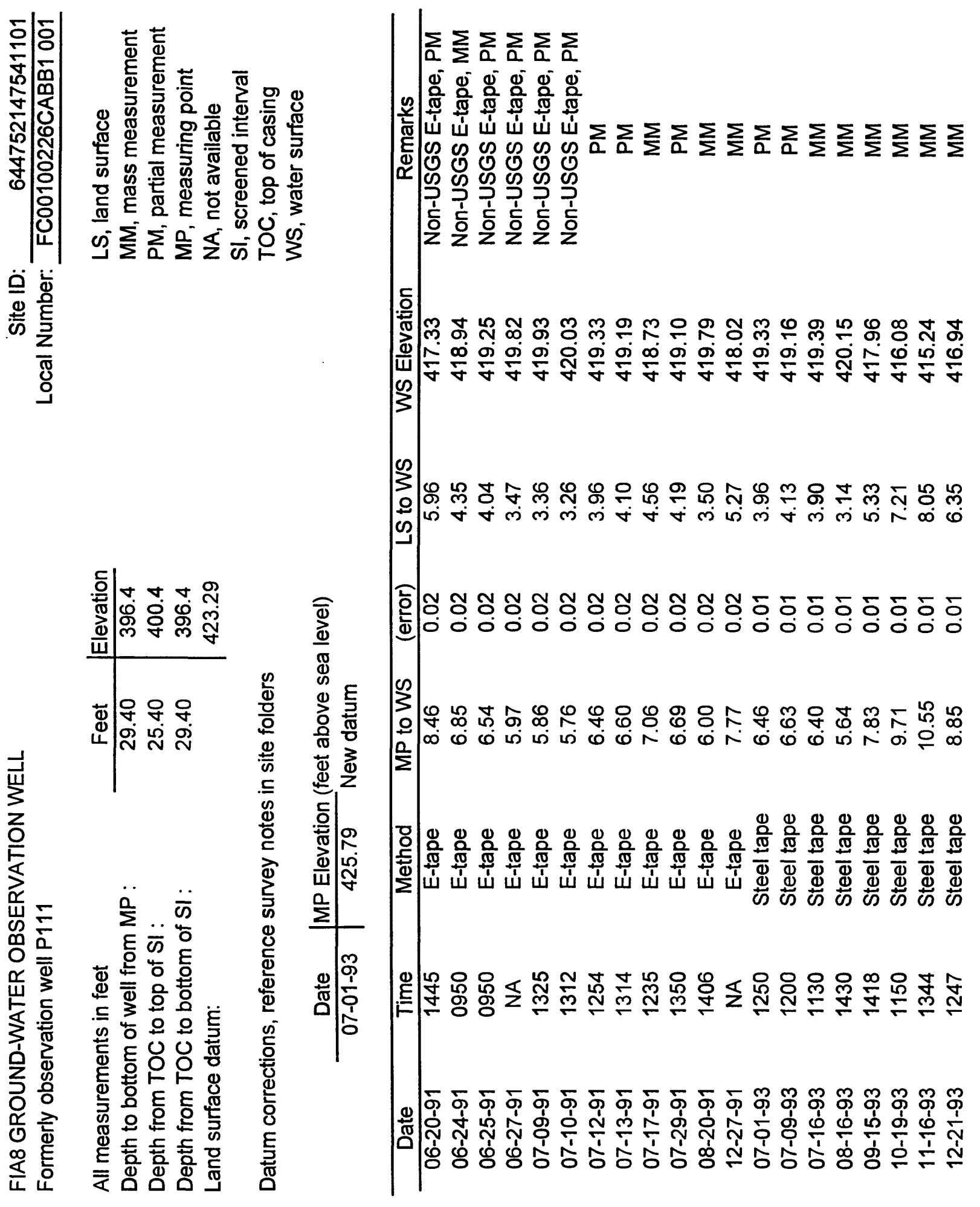


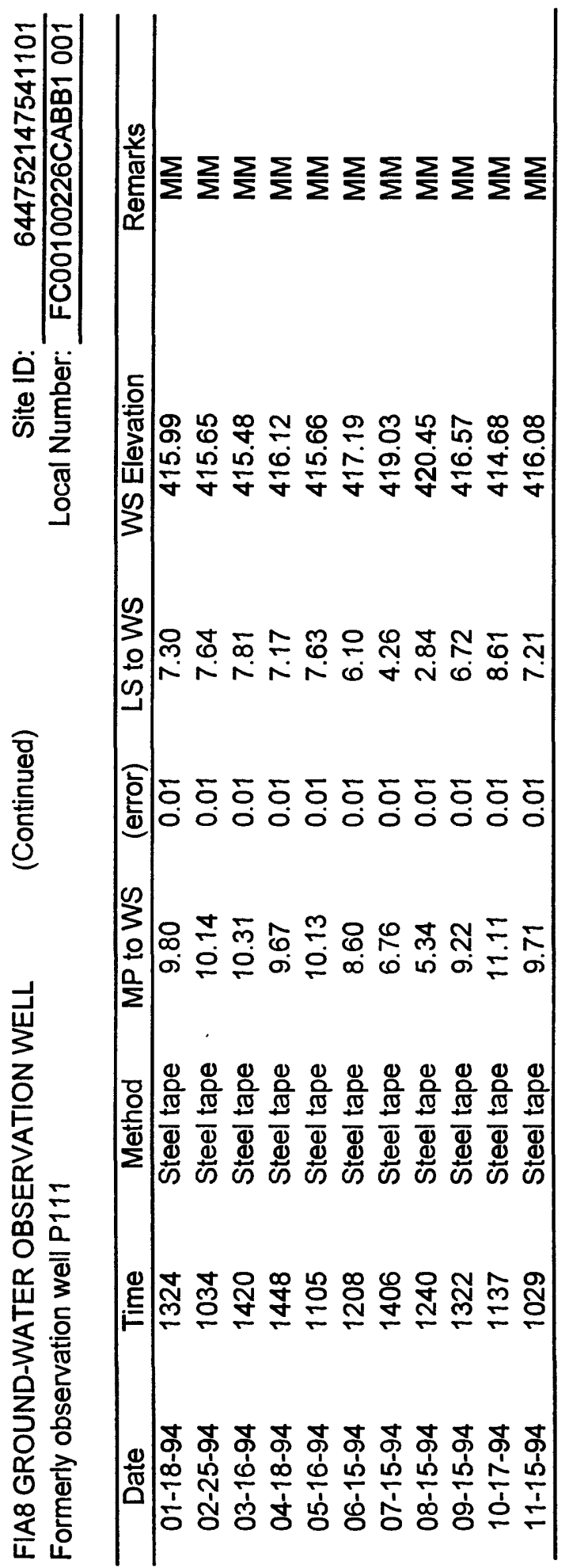




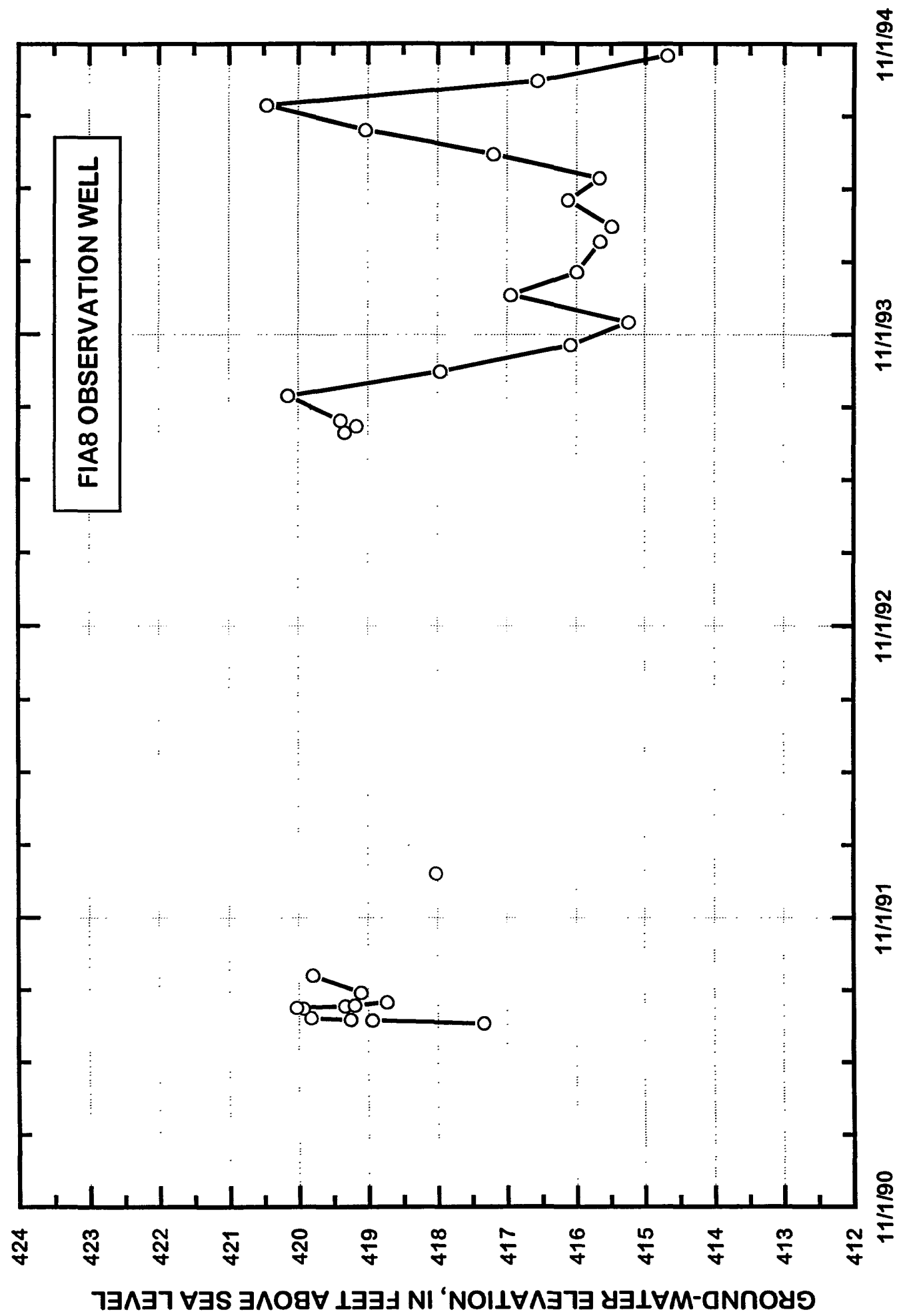



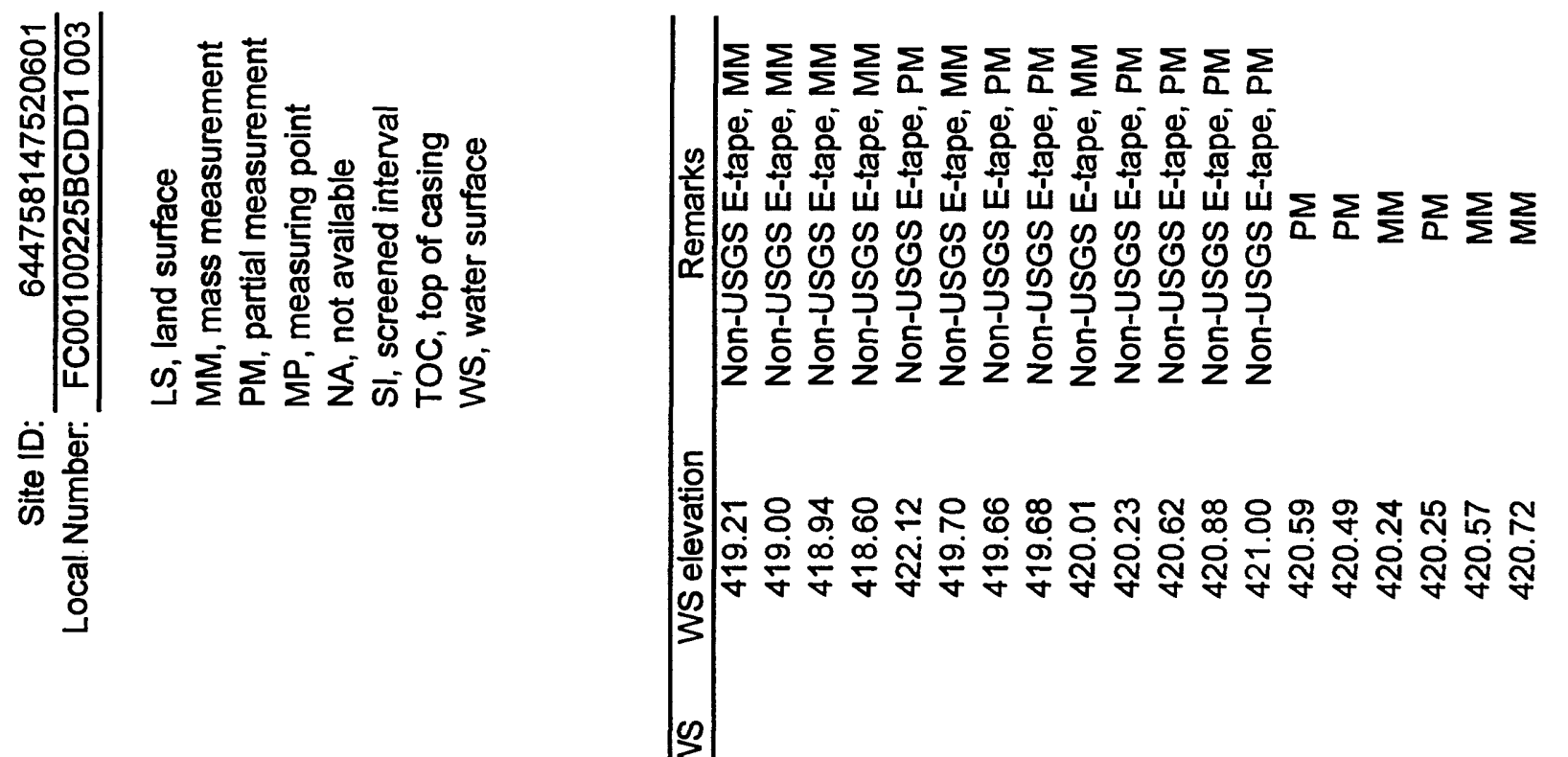

3

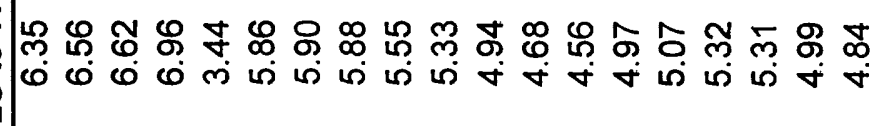

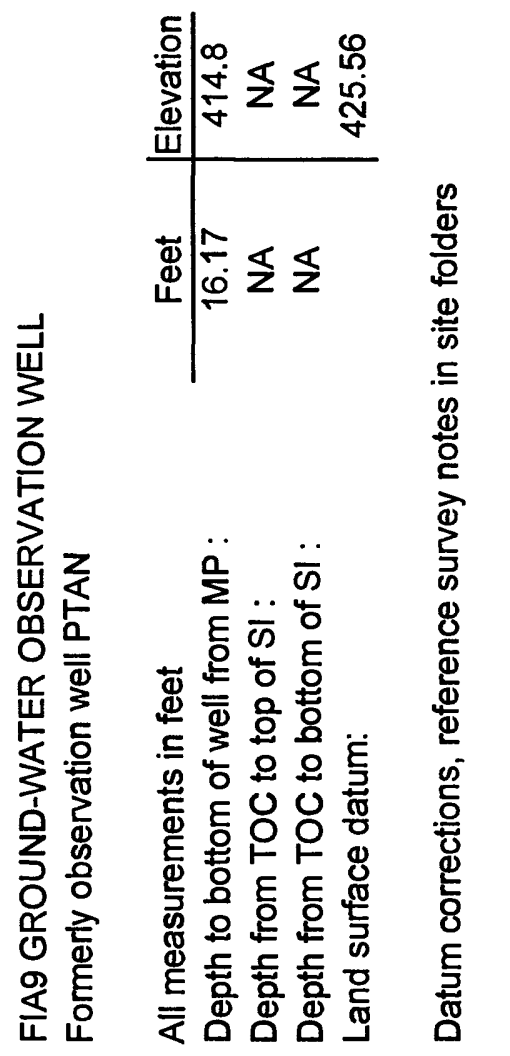

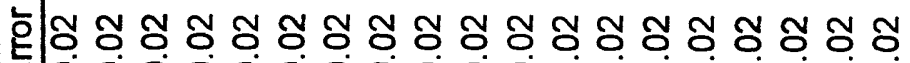

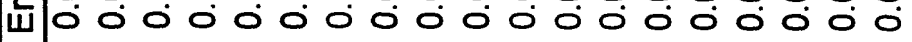

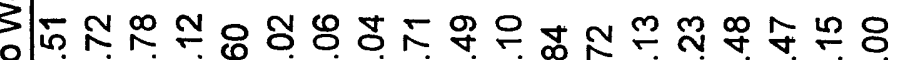

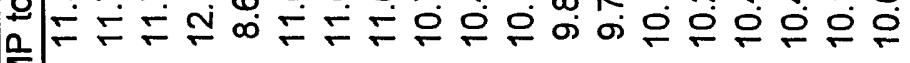

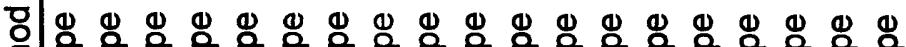

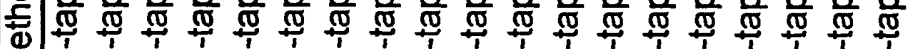
这ய

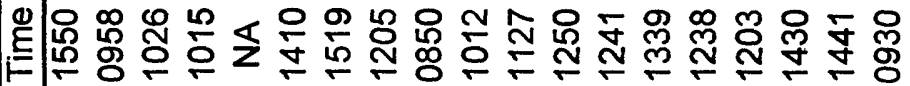

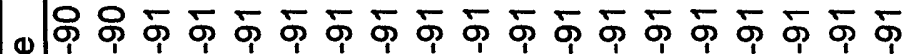
蔥

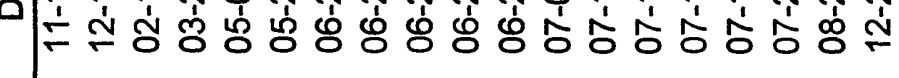




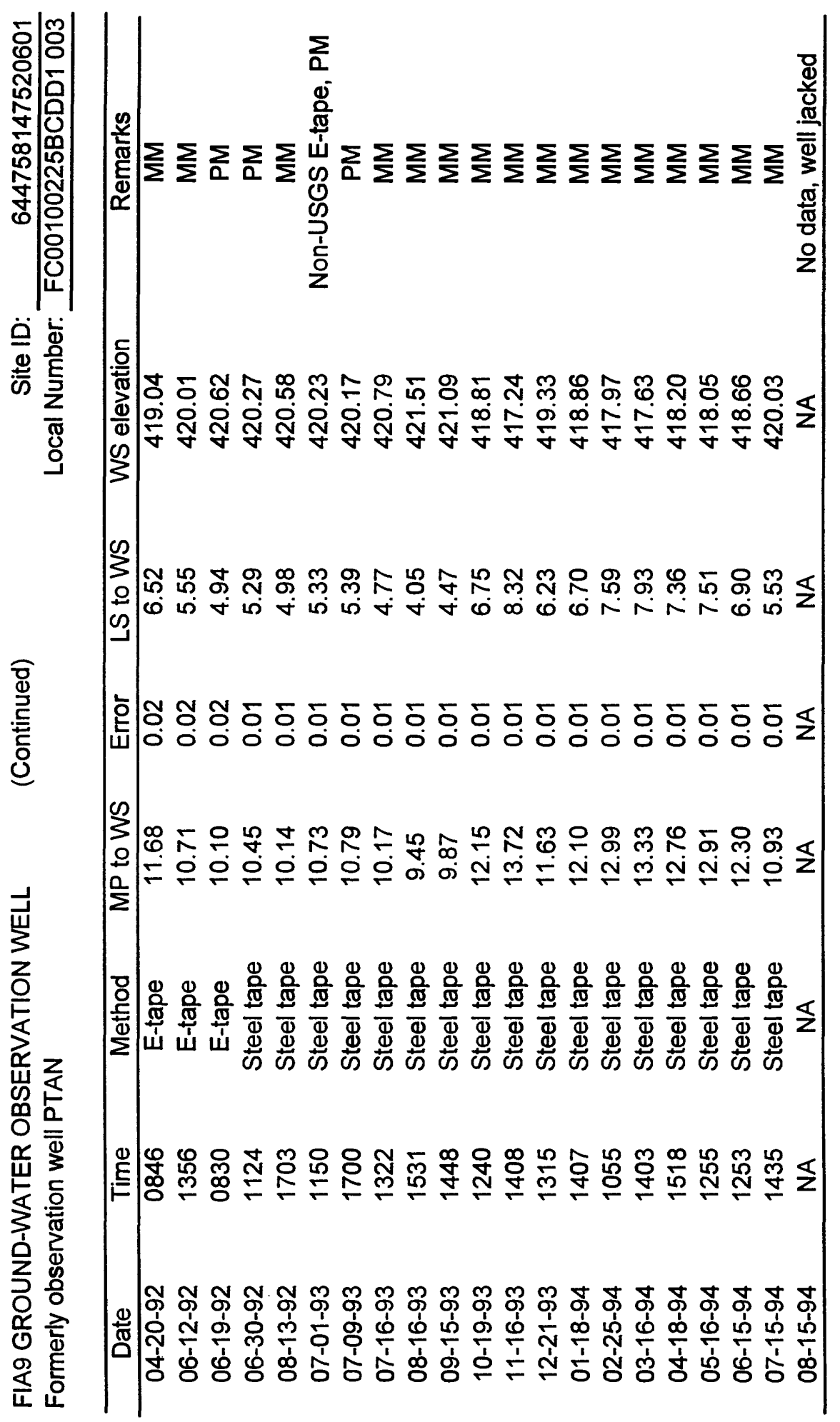




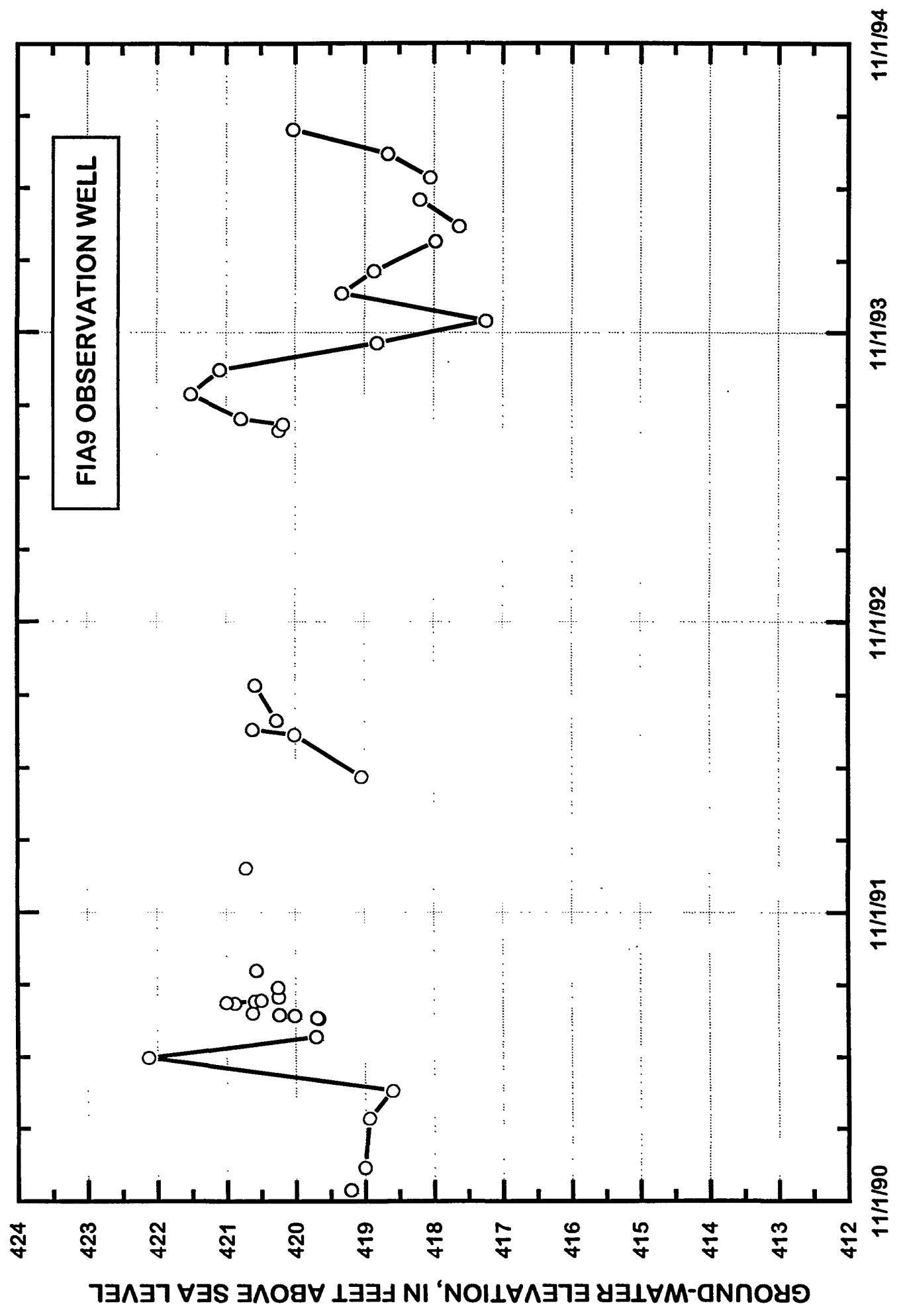




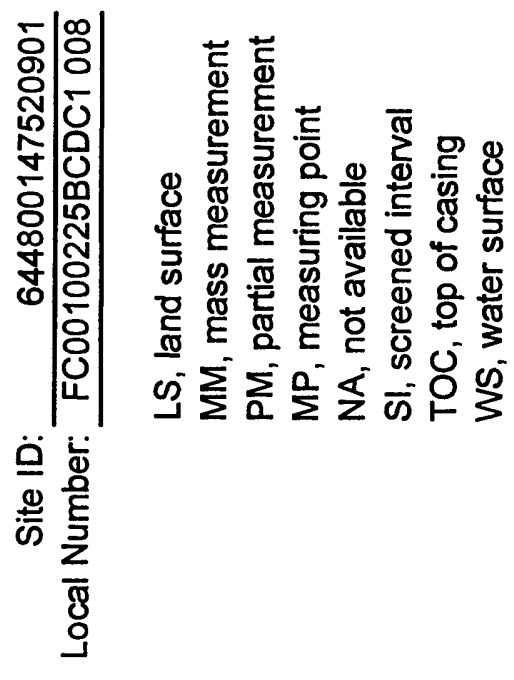

$\sum_{\sum} \sum_{0} \sum_{0} \sum_{0} \sum_{0}$

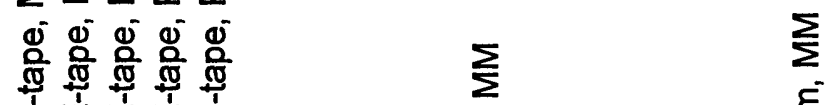

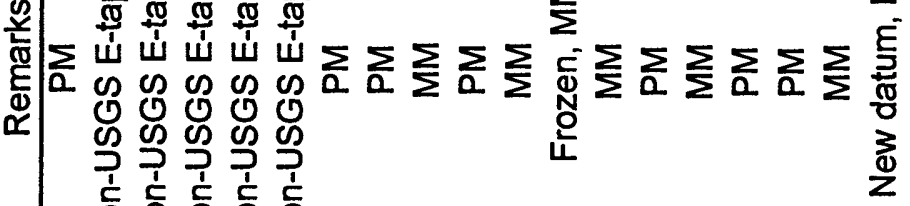

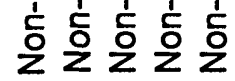

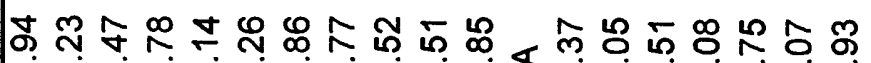

守 守

宽

क

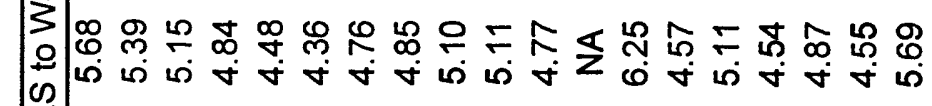

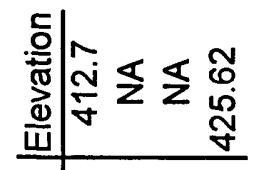

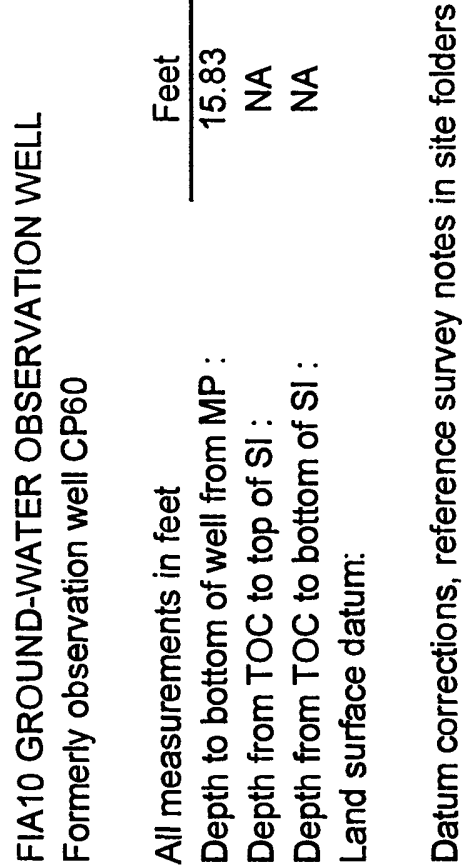

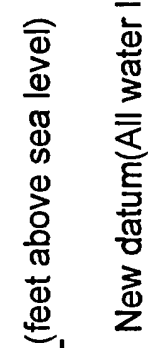

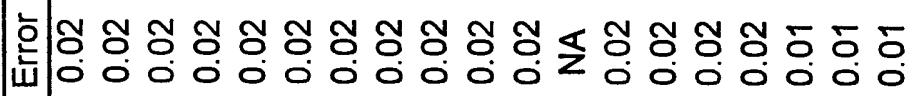

$\infty$
3
0
0
$\frac{1}{\Sigma}$

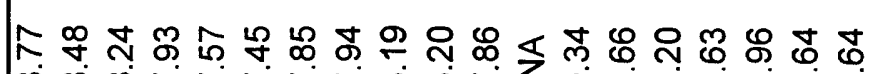

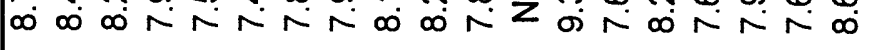

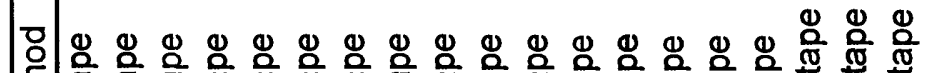

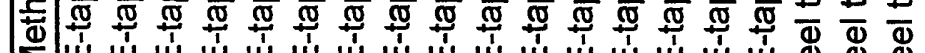

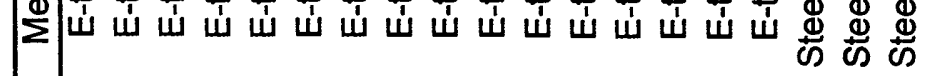

号

㞧

๑) \%

ํㅣㄴ

范

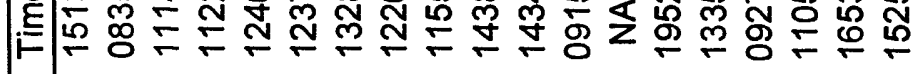

ڤ́⿺

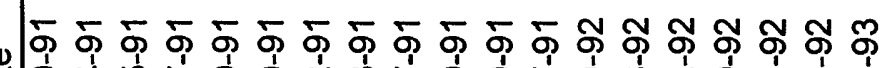

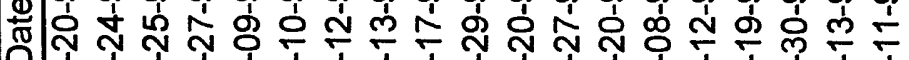

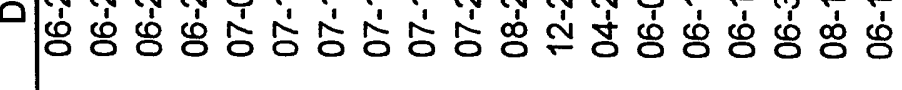




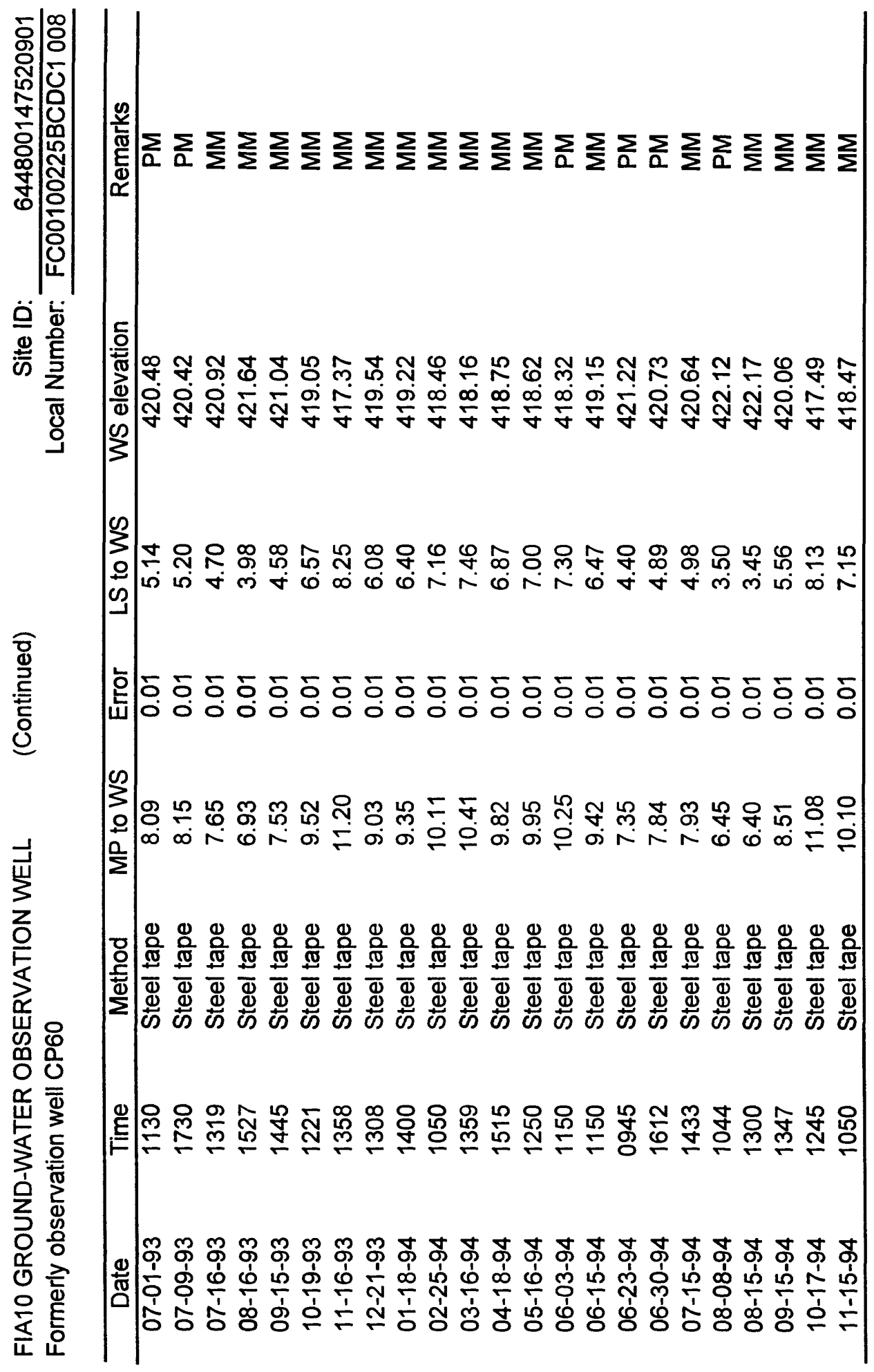




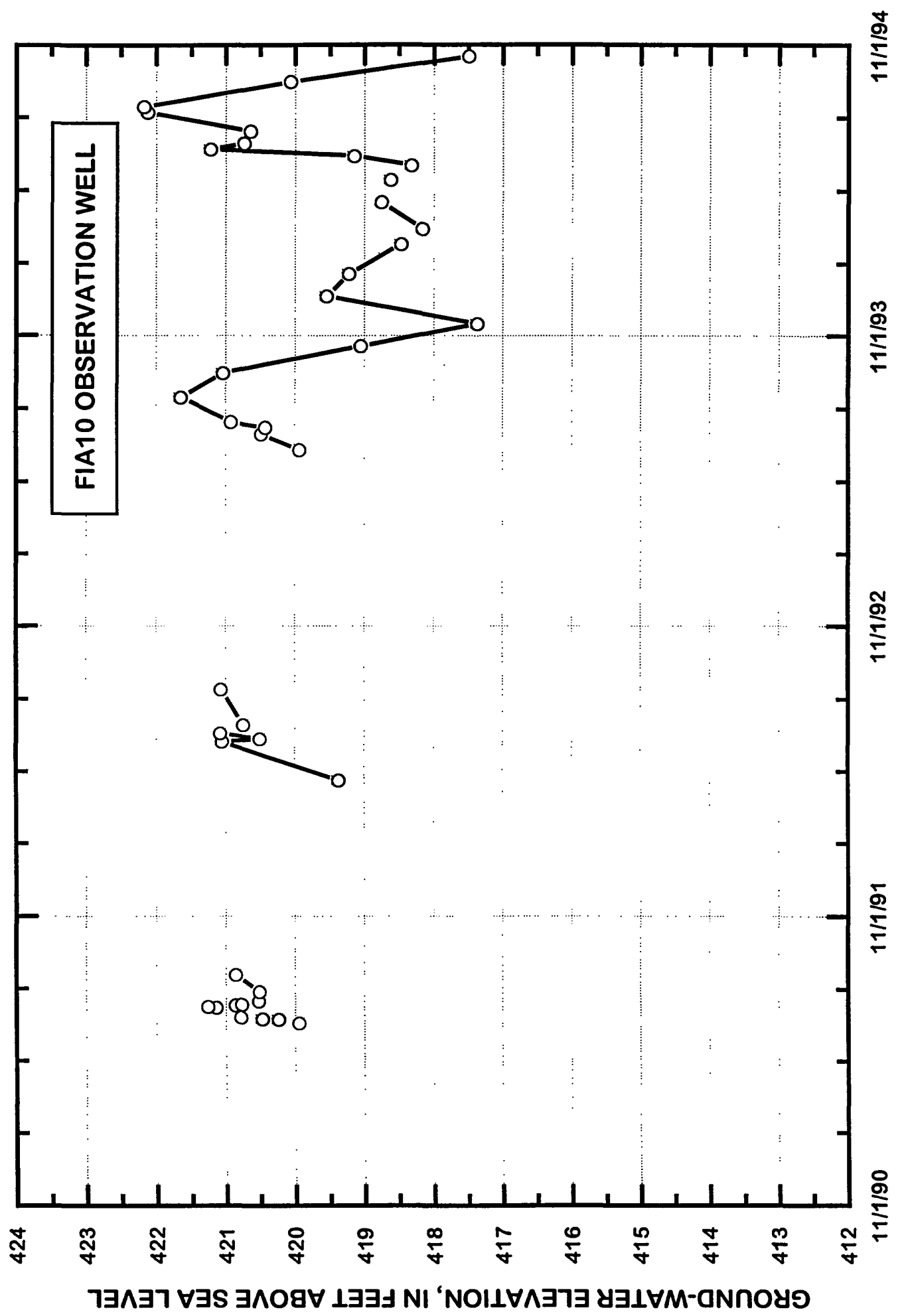




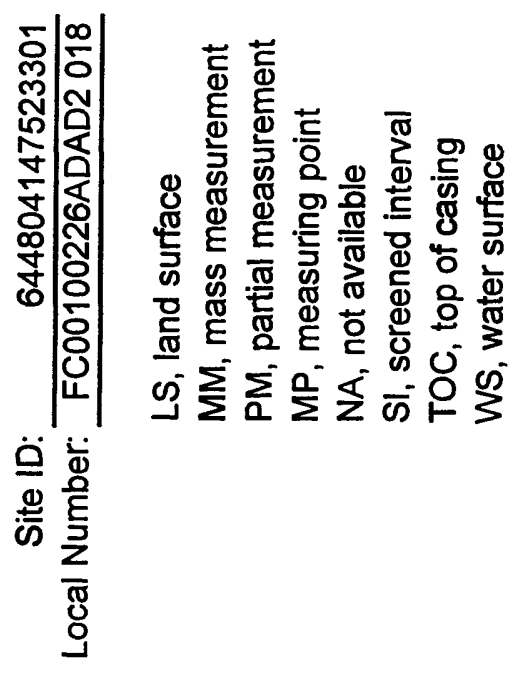

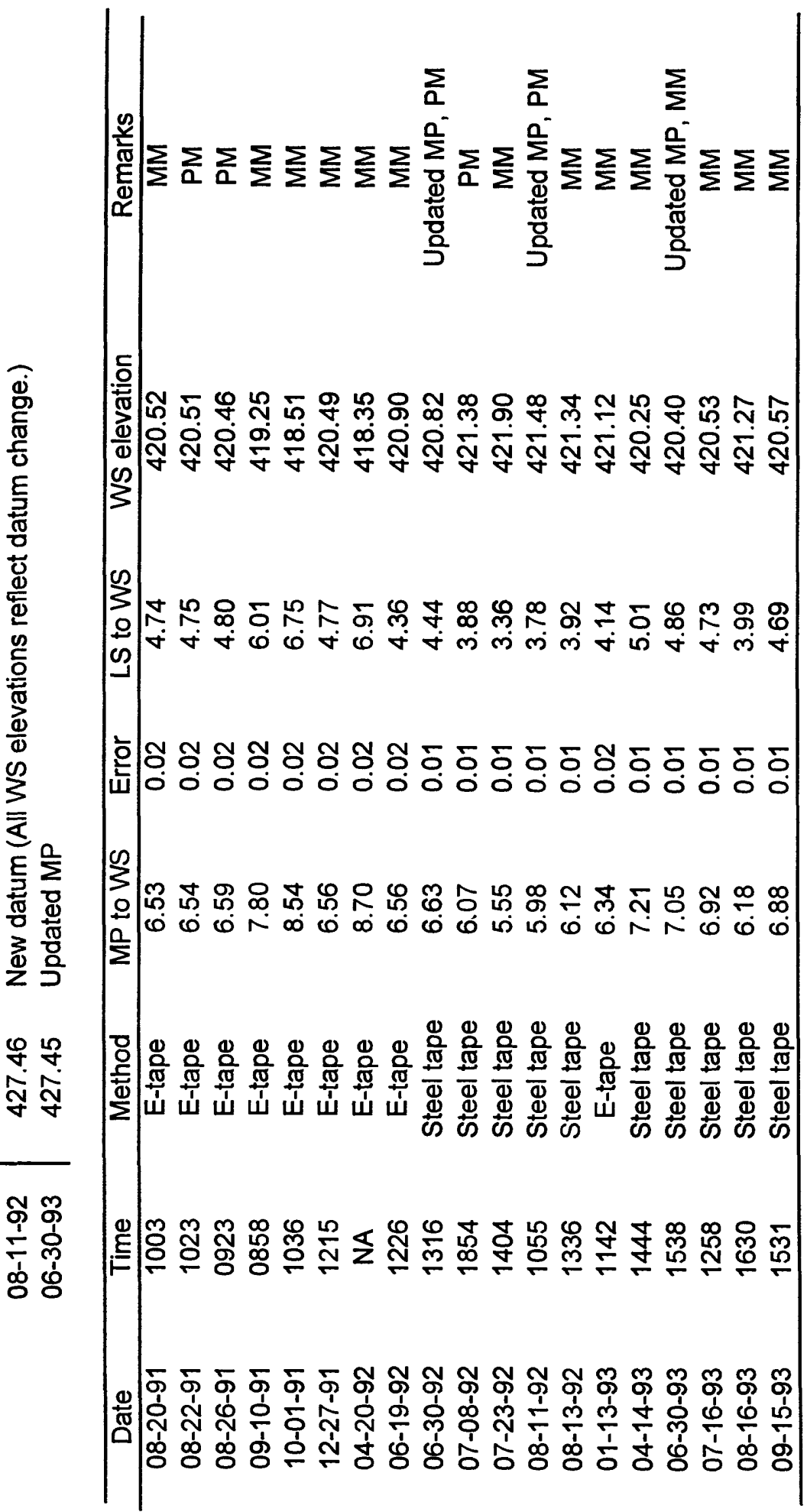

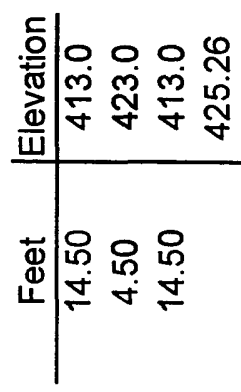

$\frac{0}{0}$
$\frac{0}{0}$
$\frac{0}{0}$
$\frac{9}{\omega}$
.5

z

을

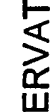

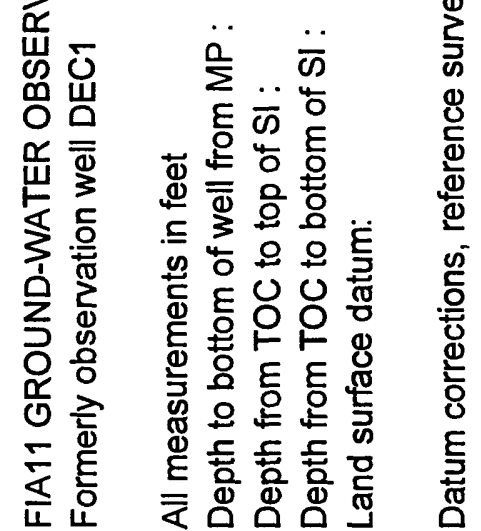

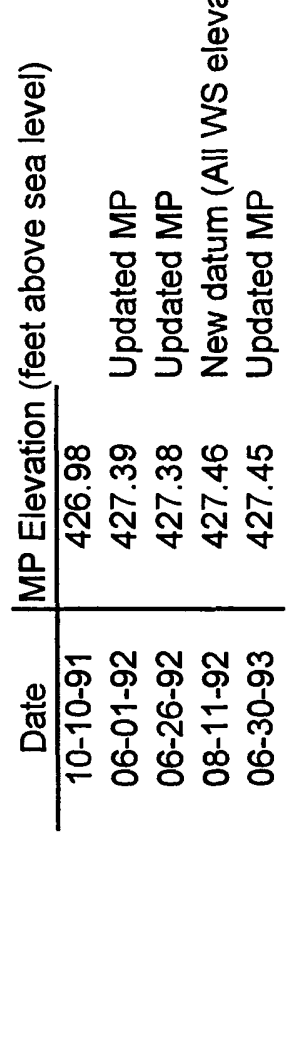




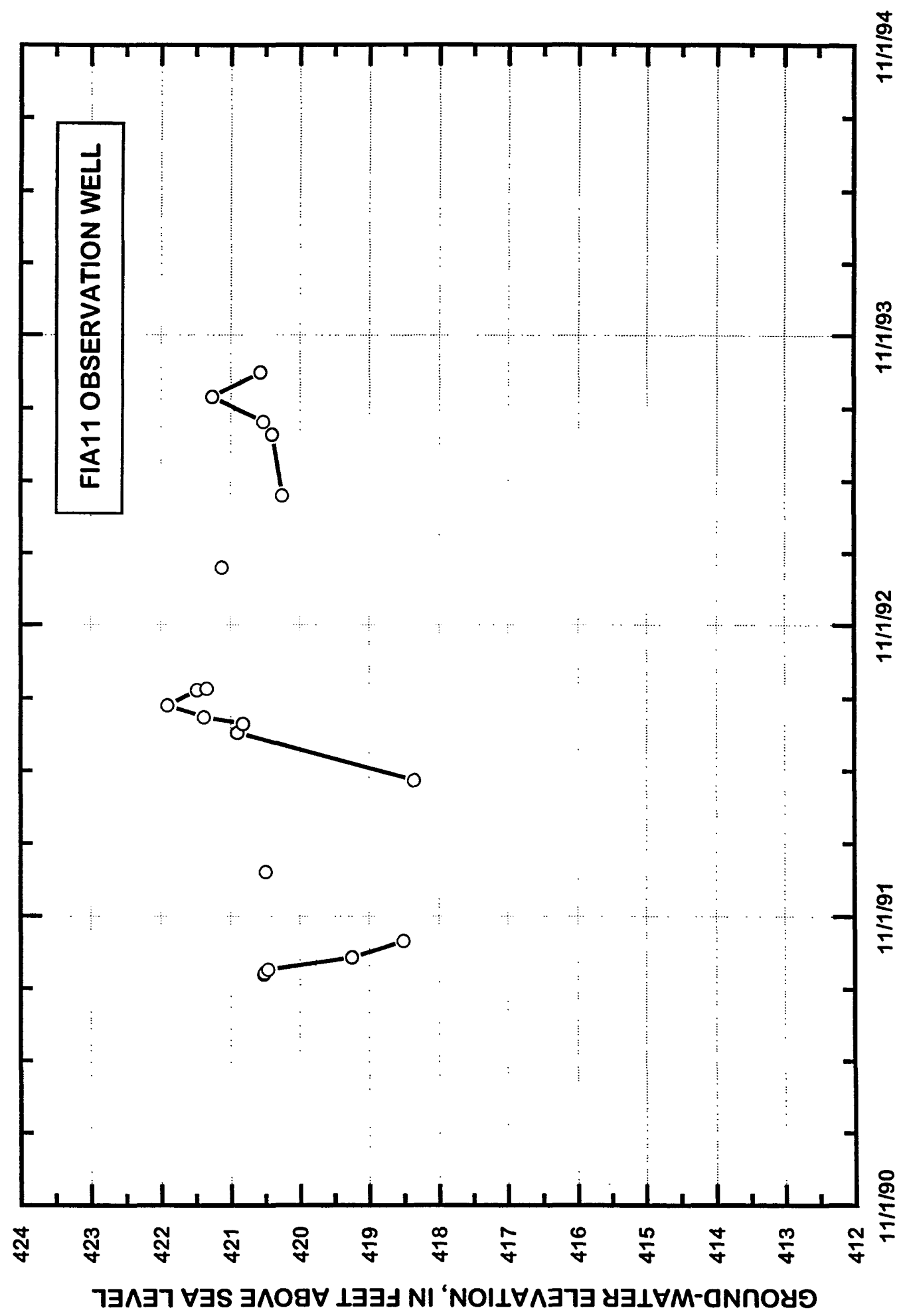



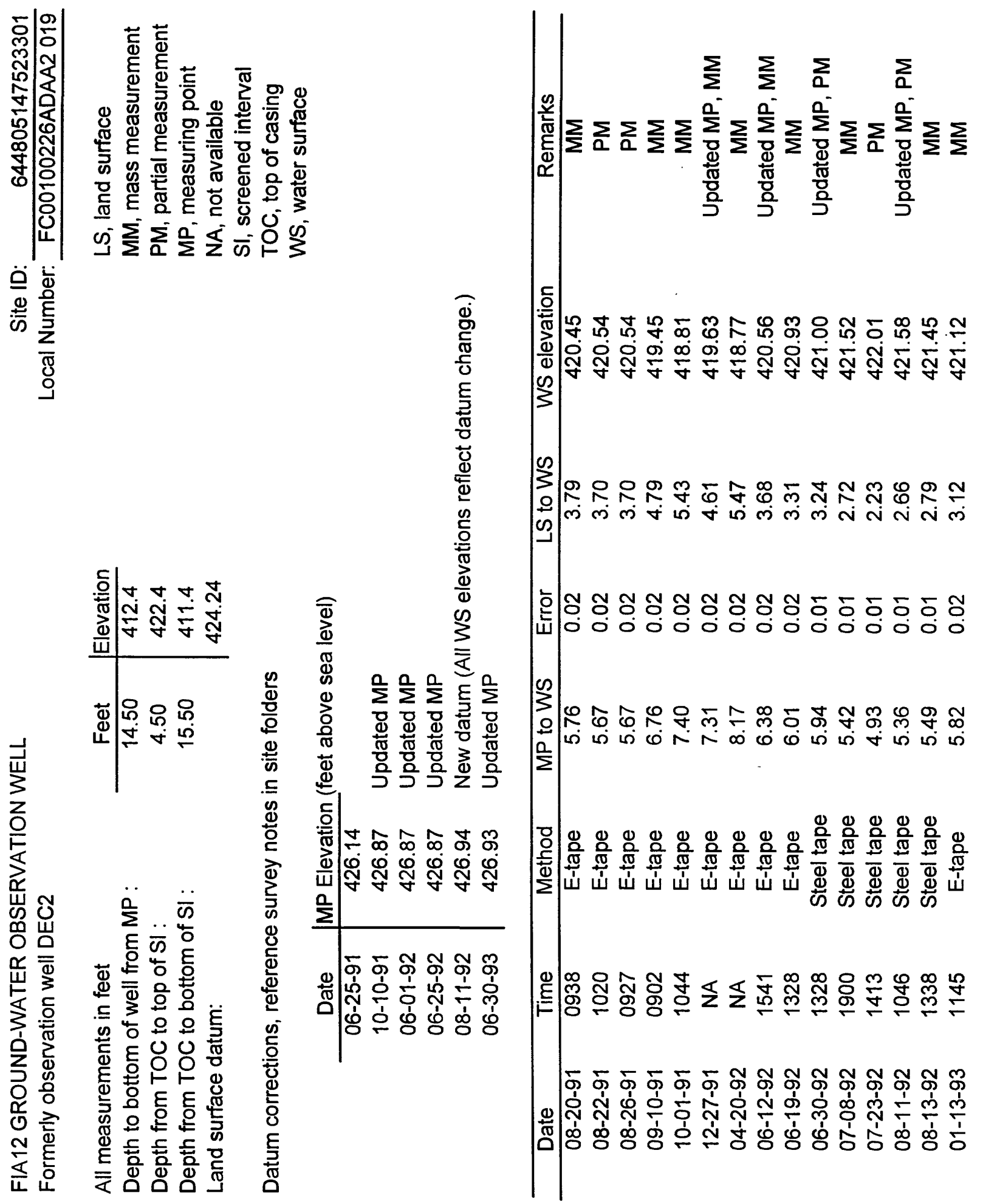


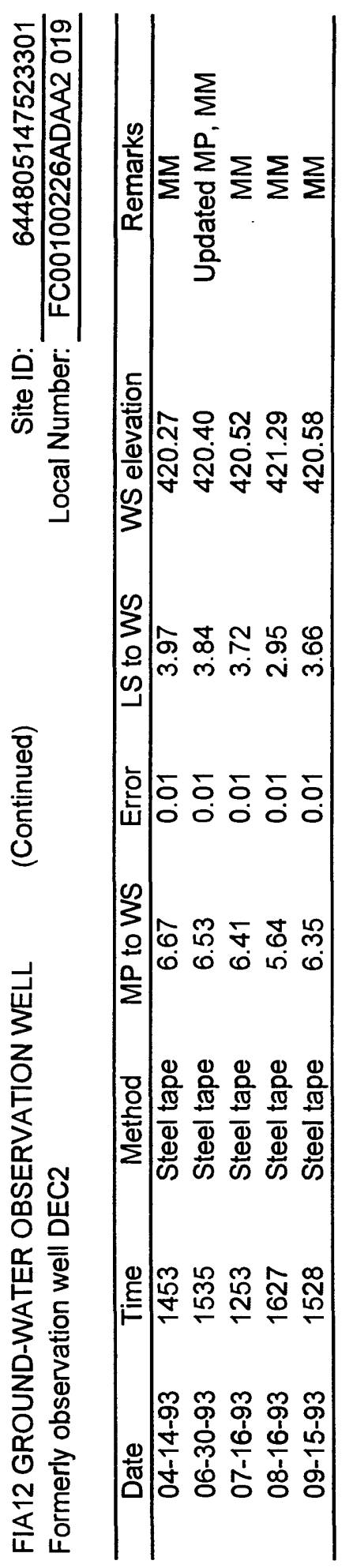




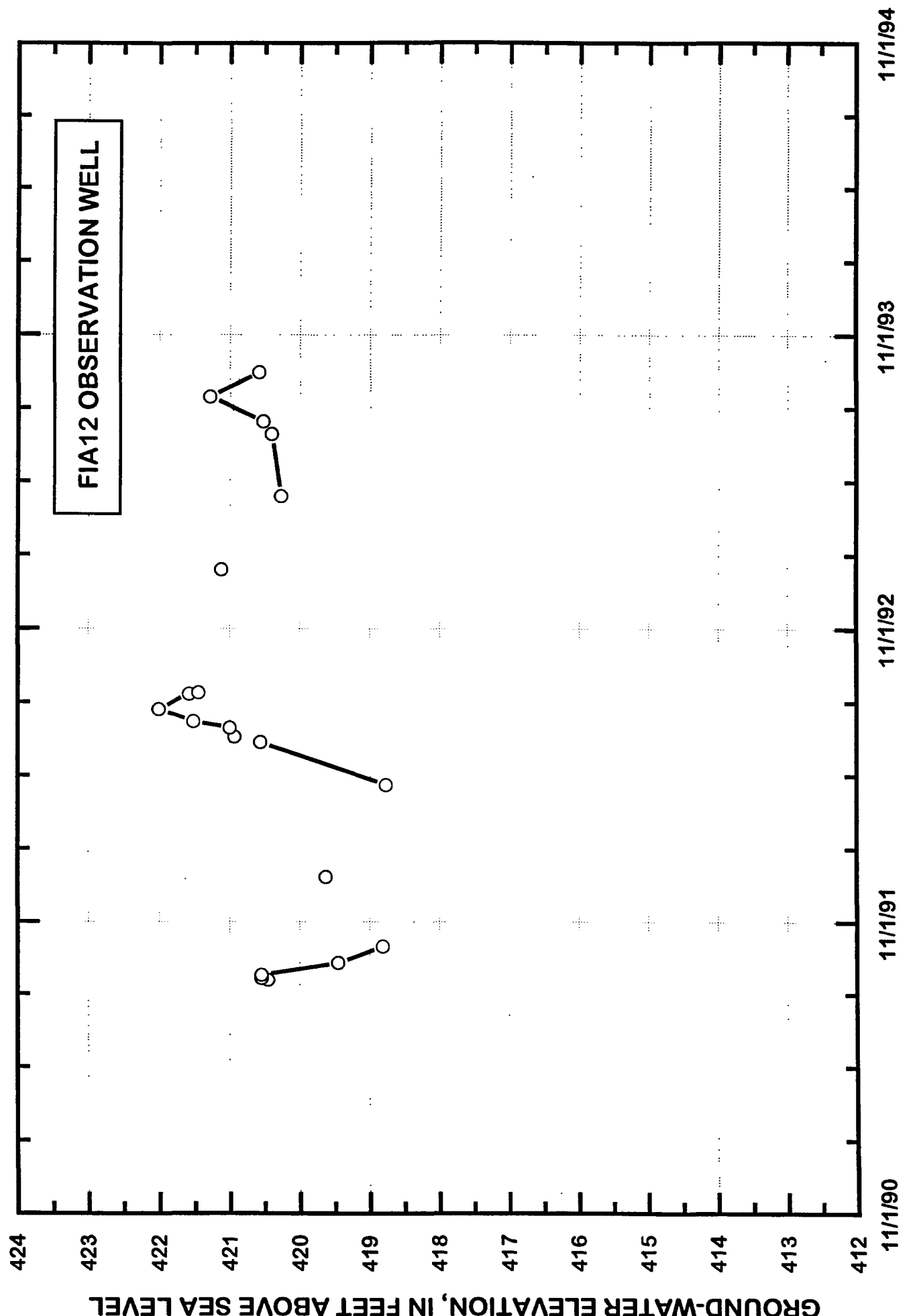



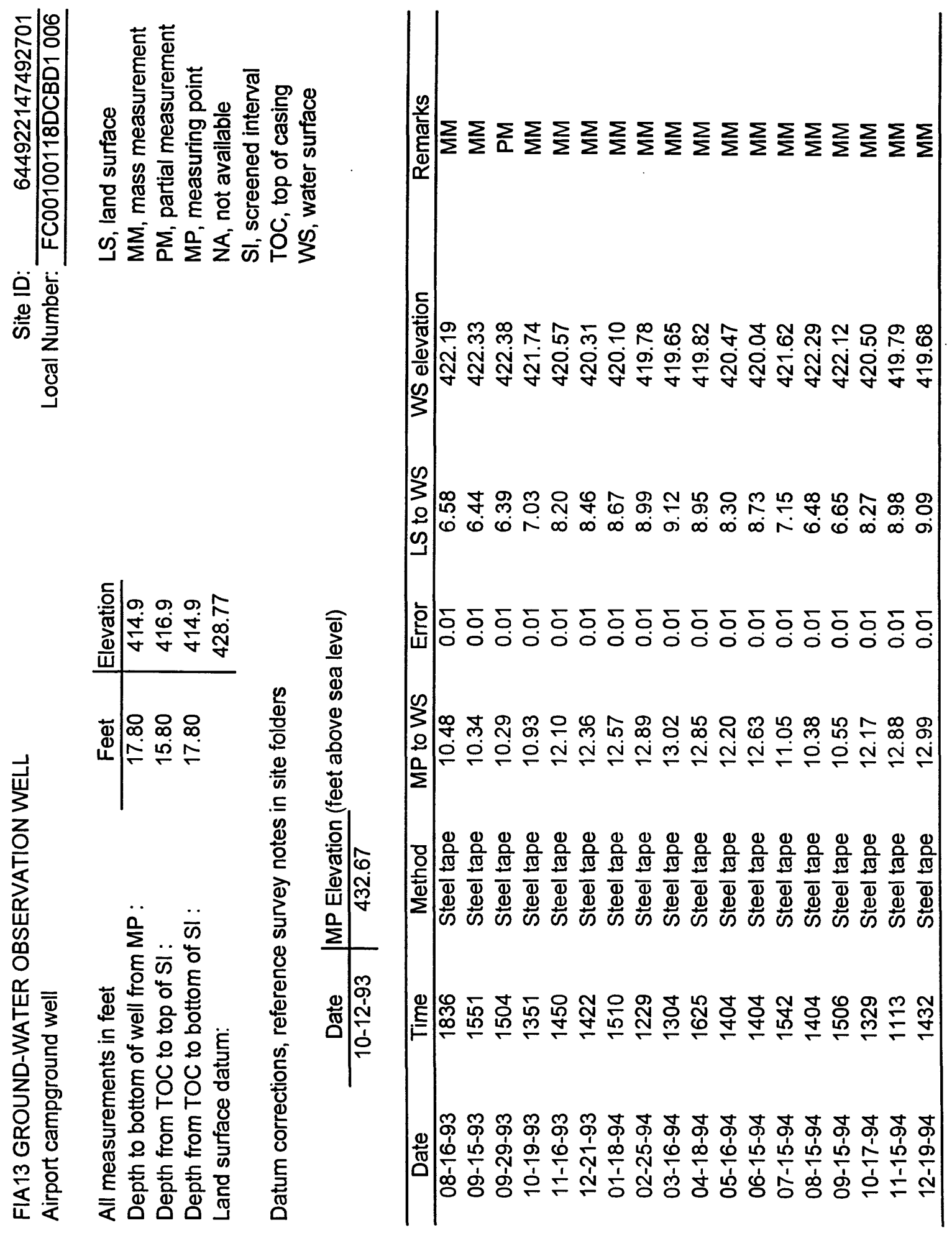


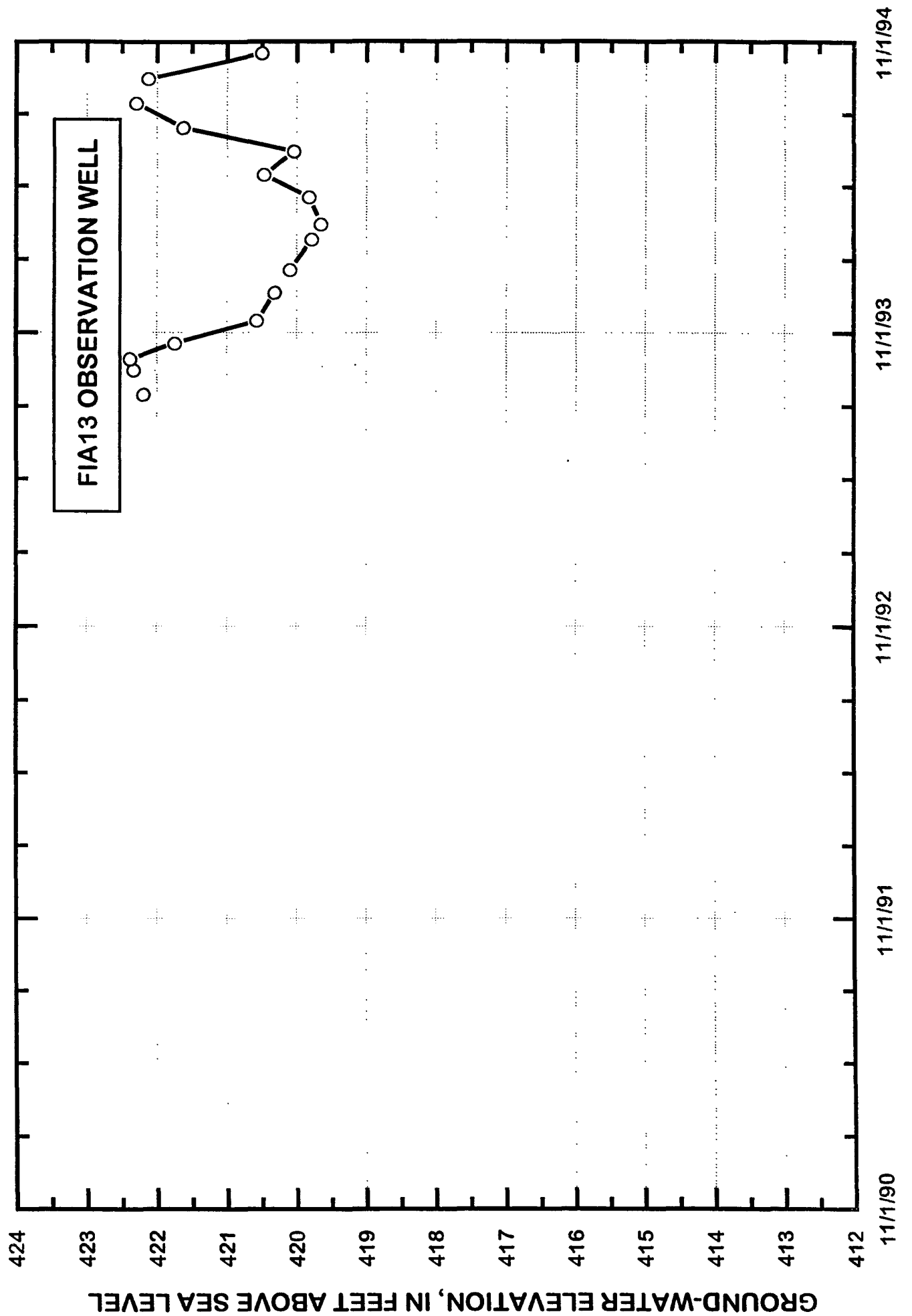




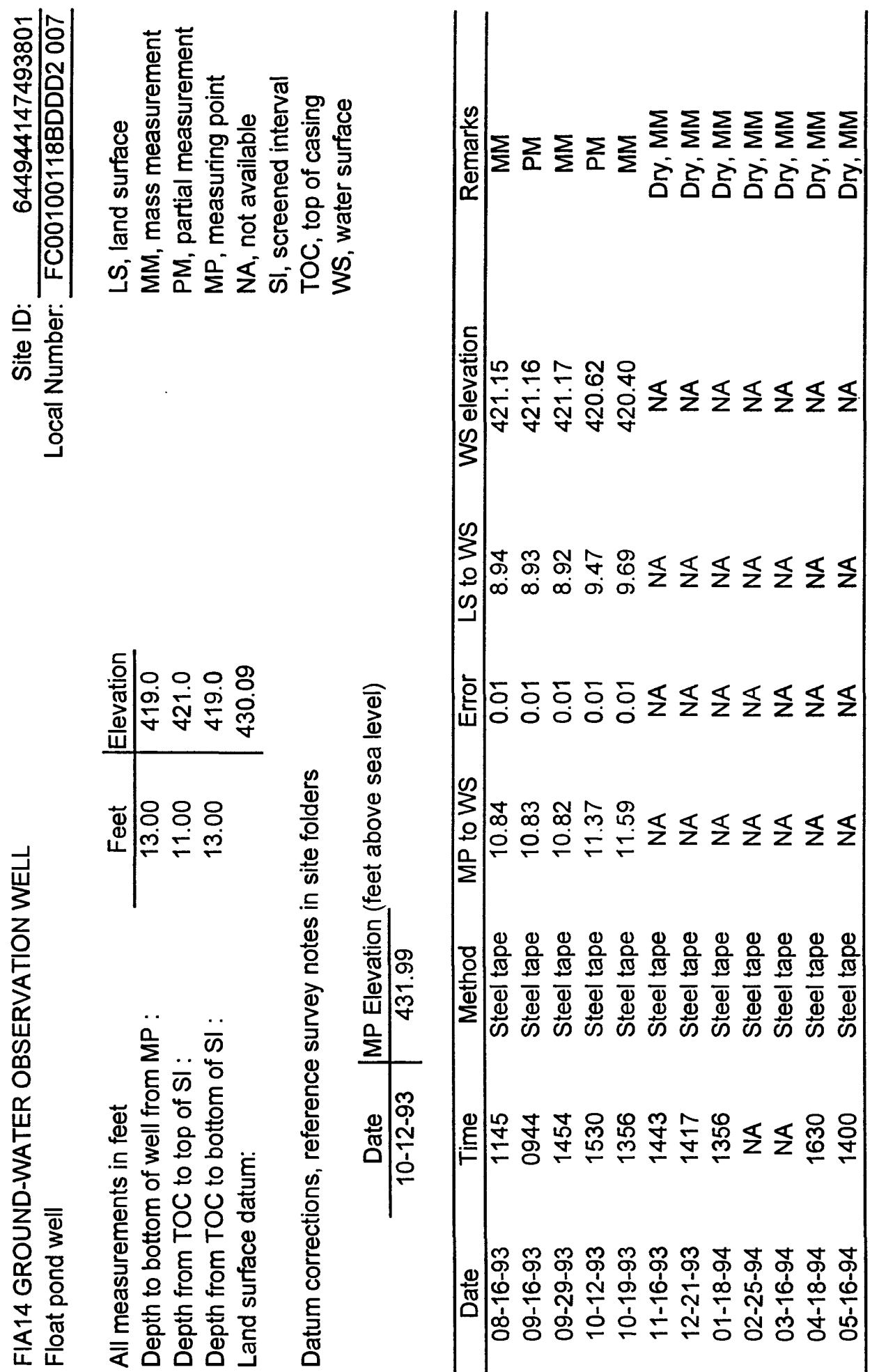




$$
17
$$



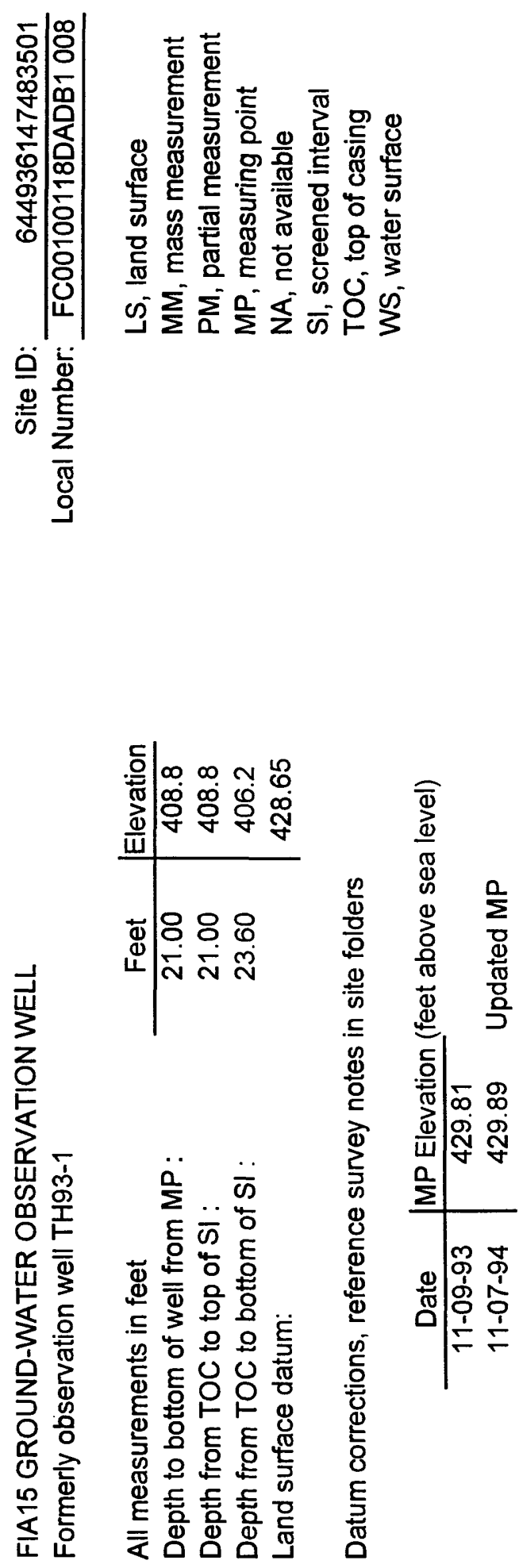

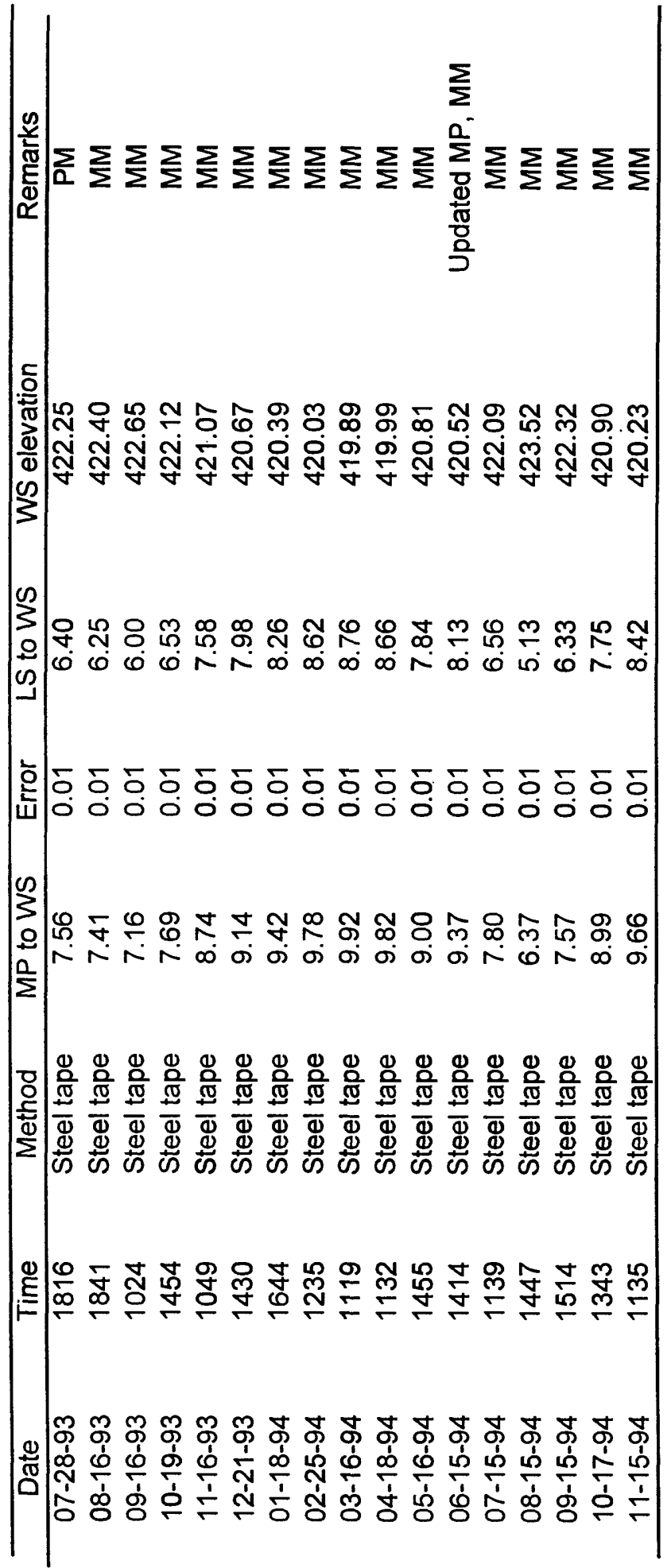




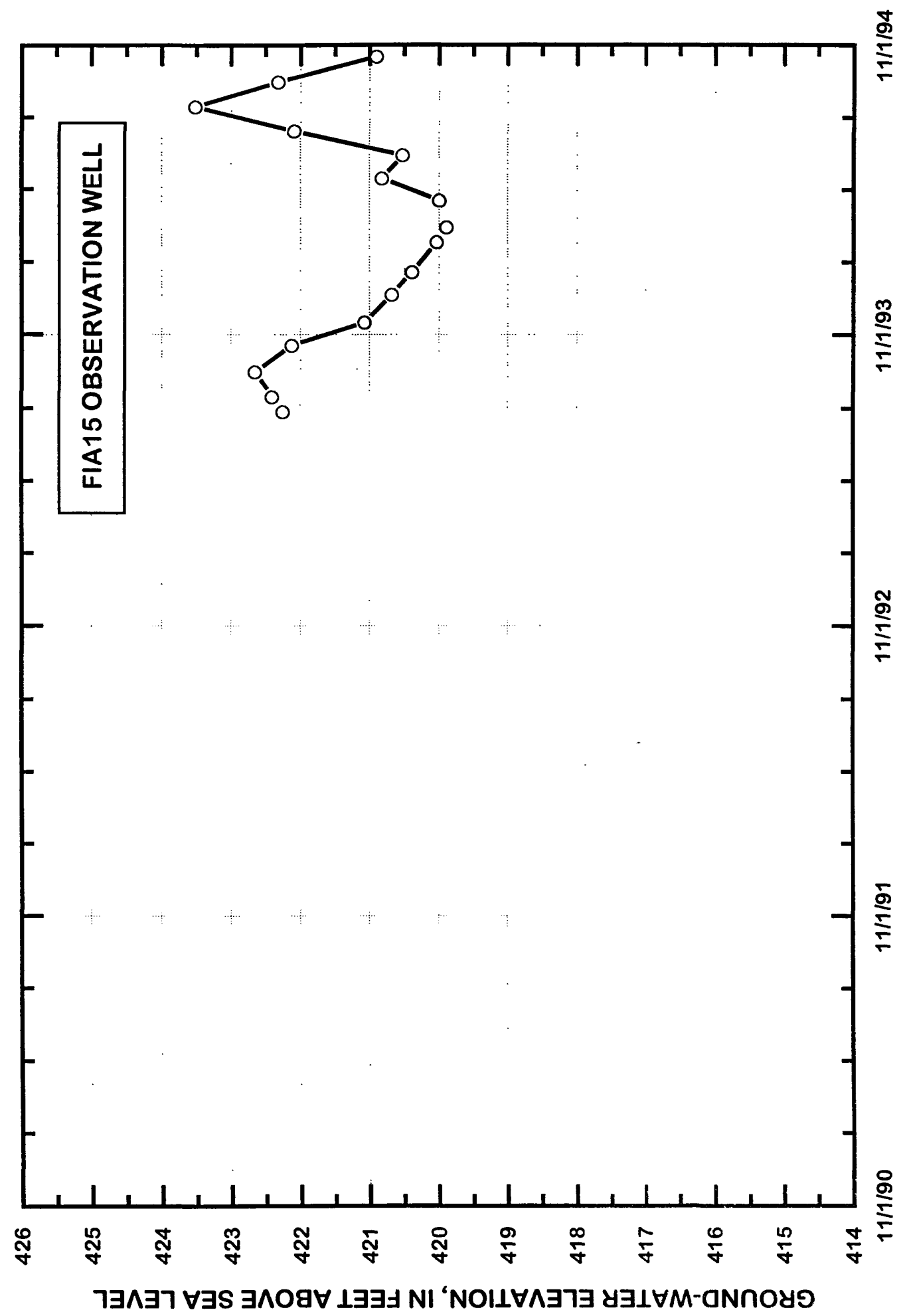




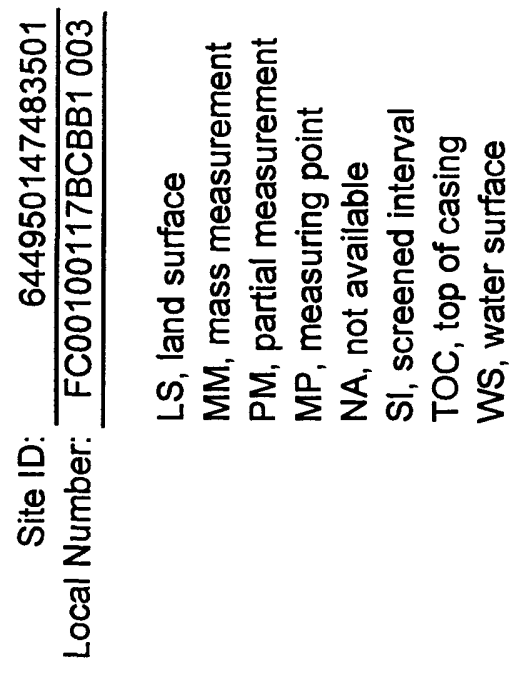

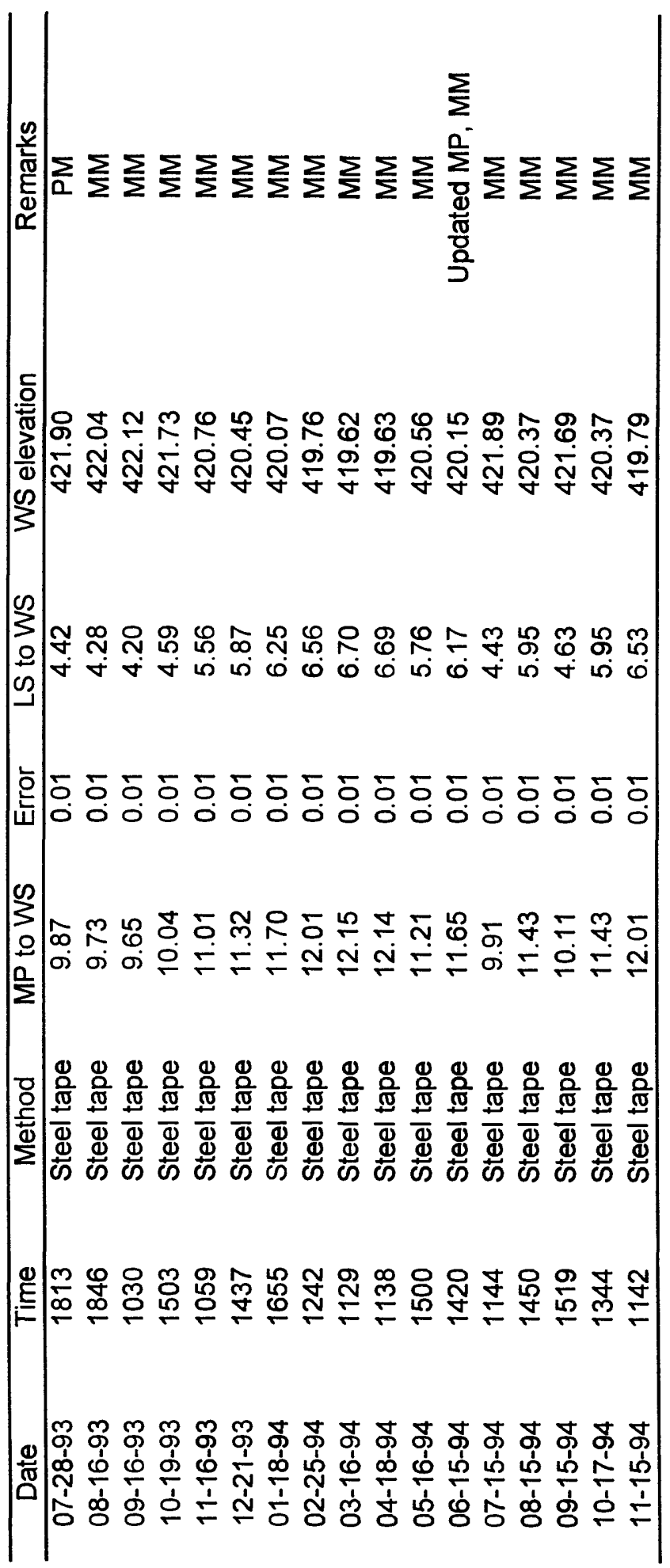




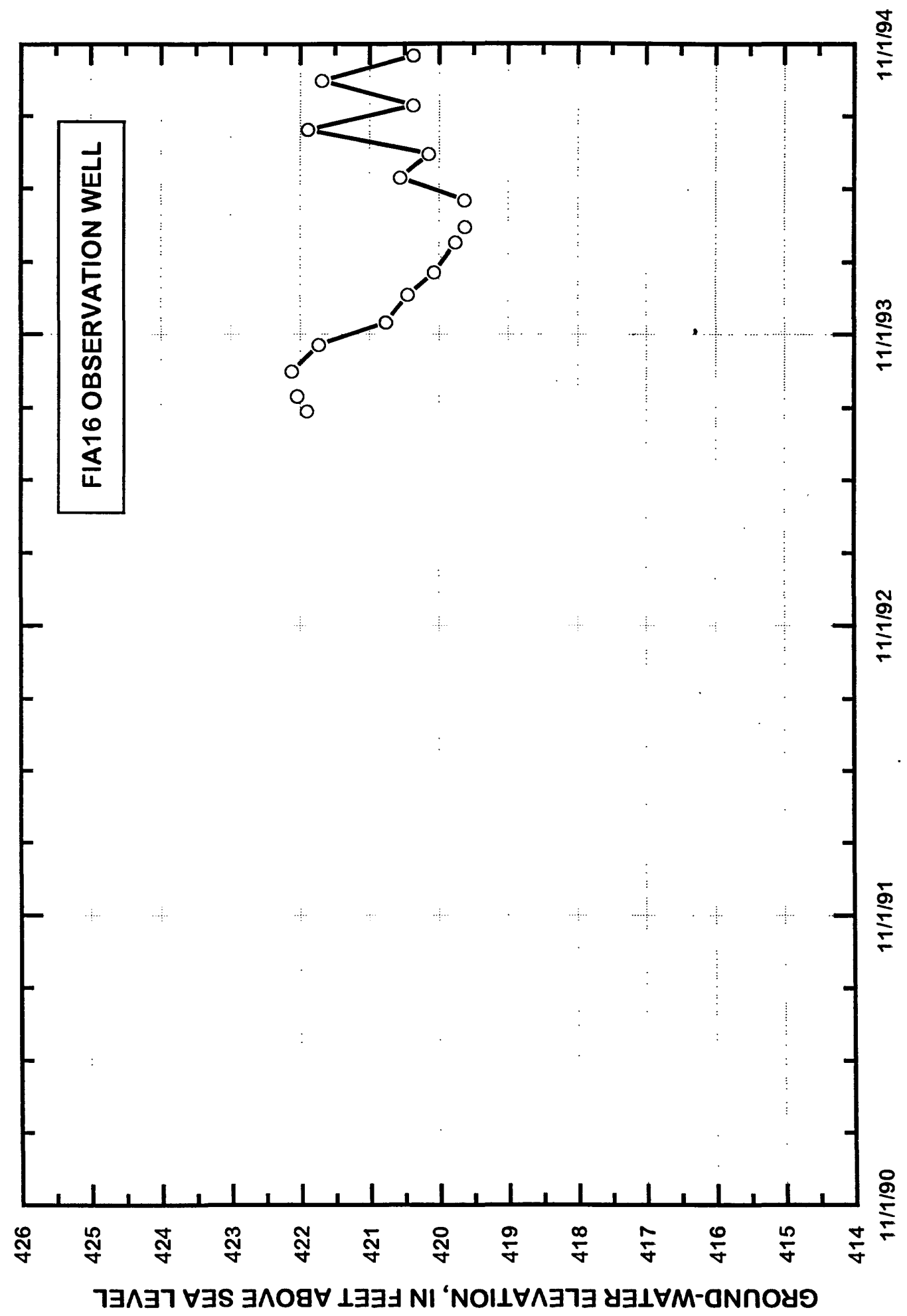



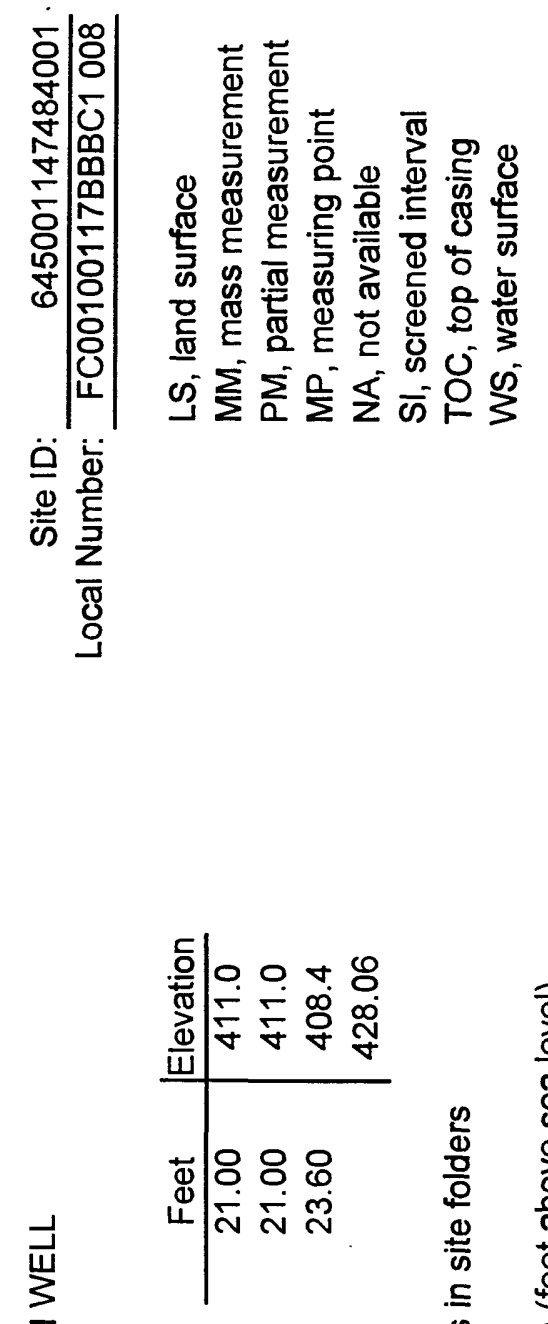

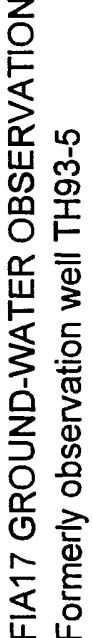

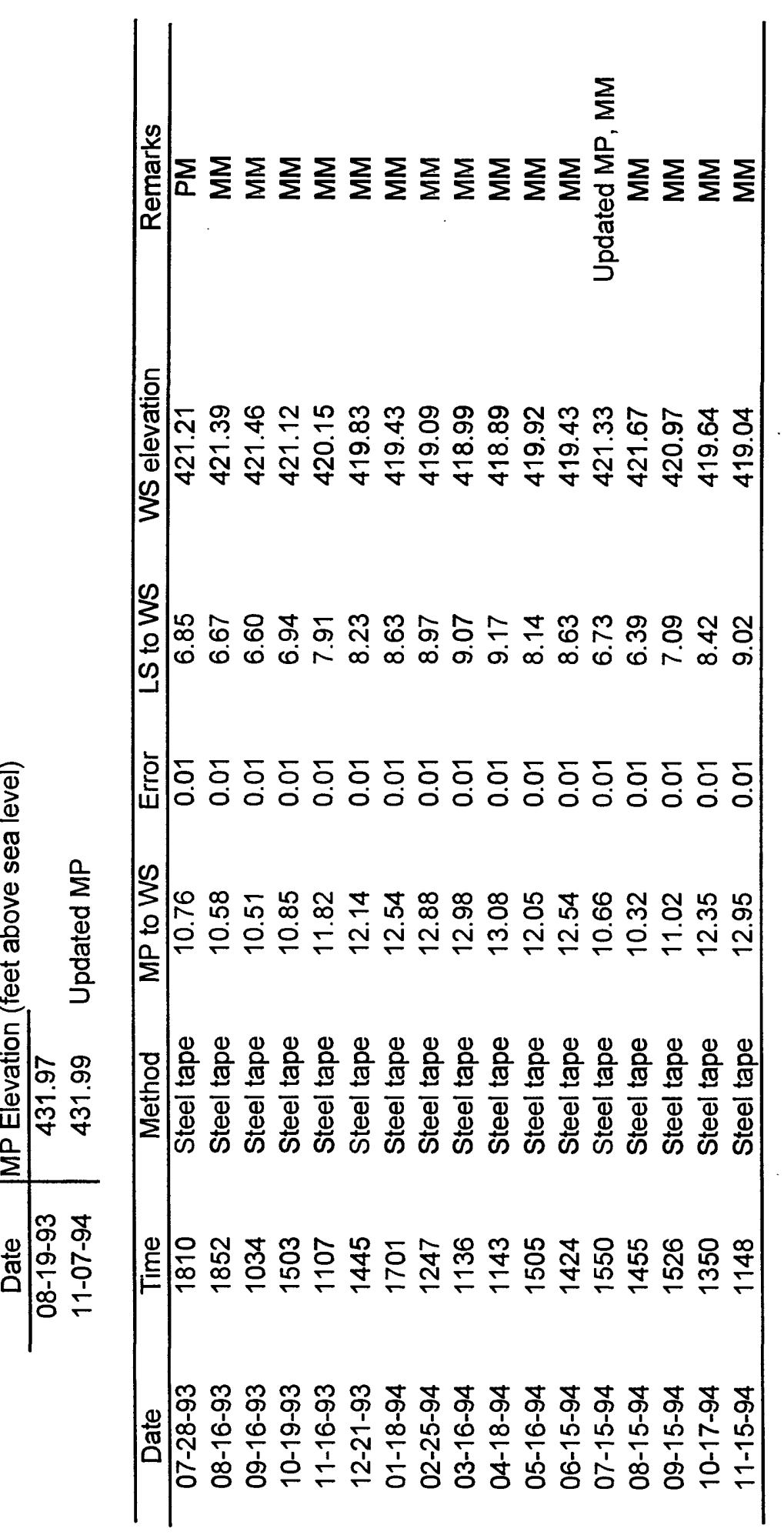




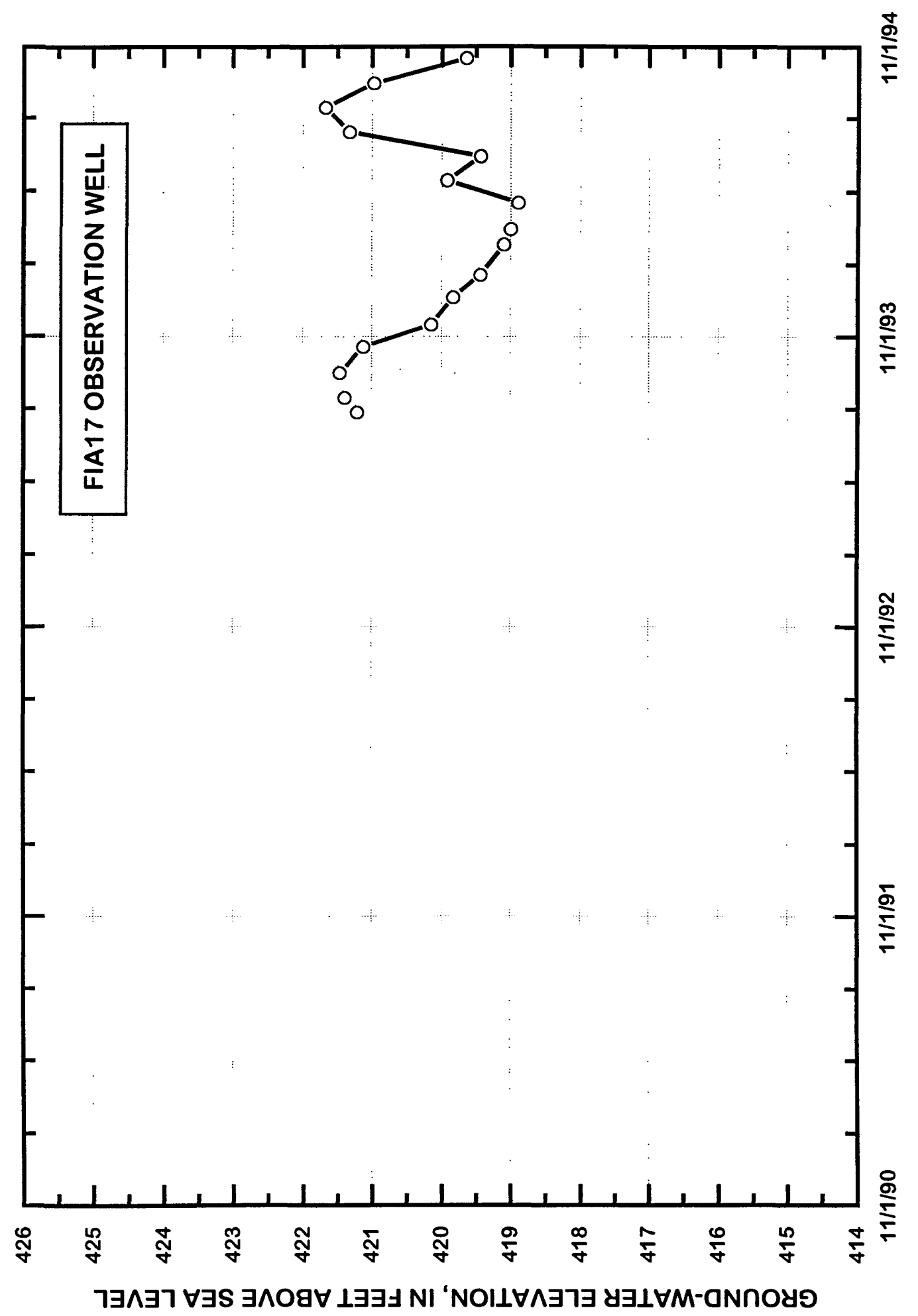



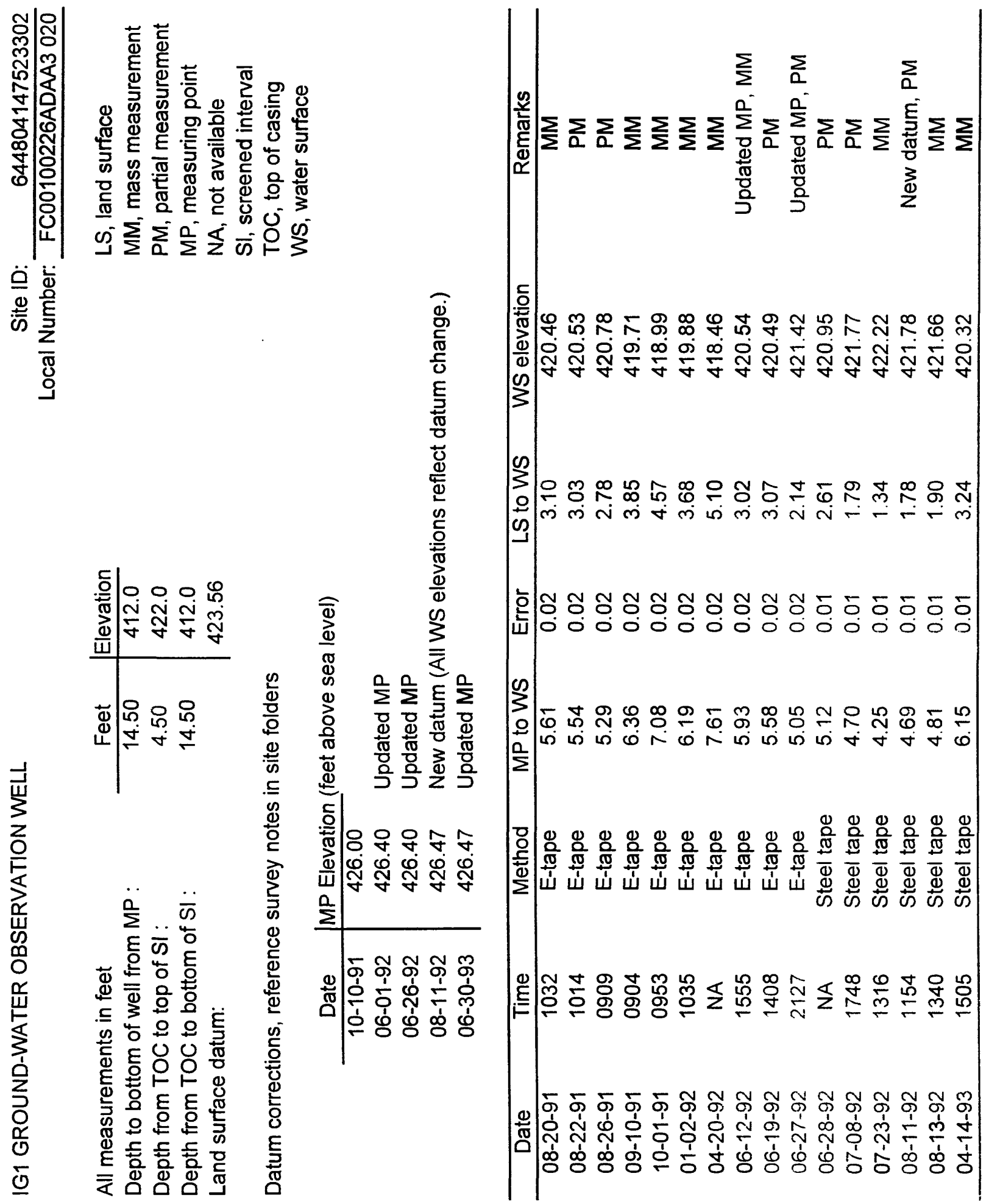


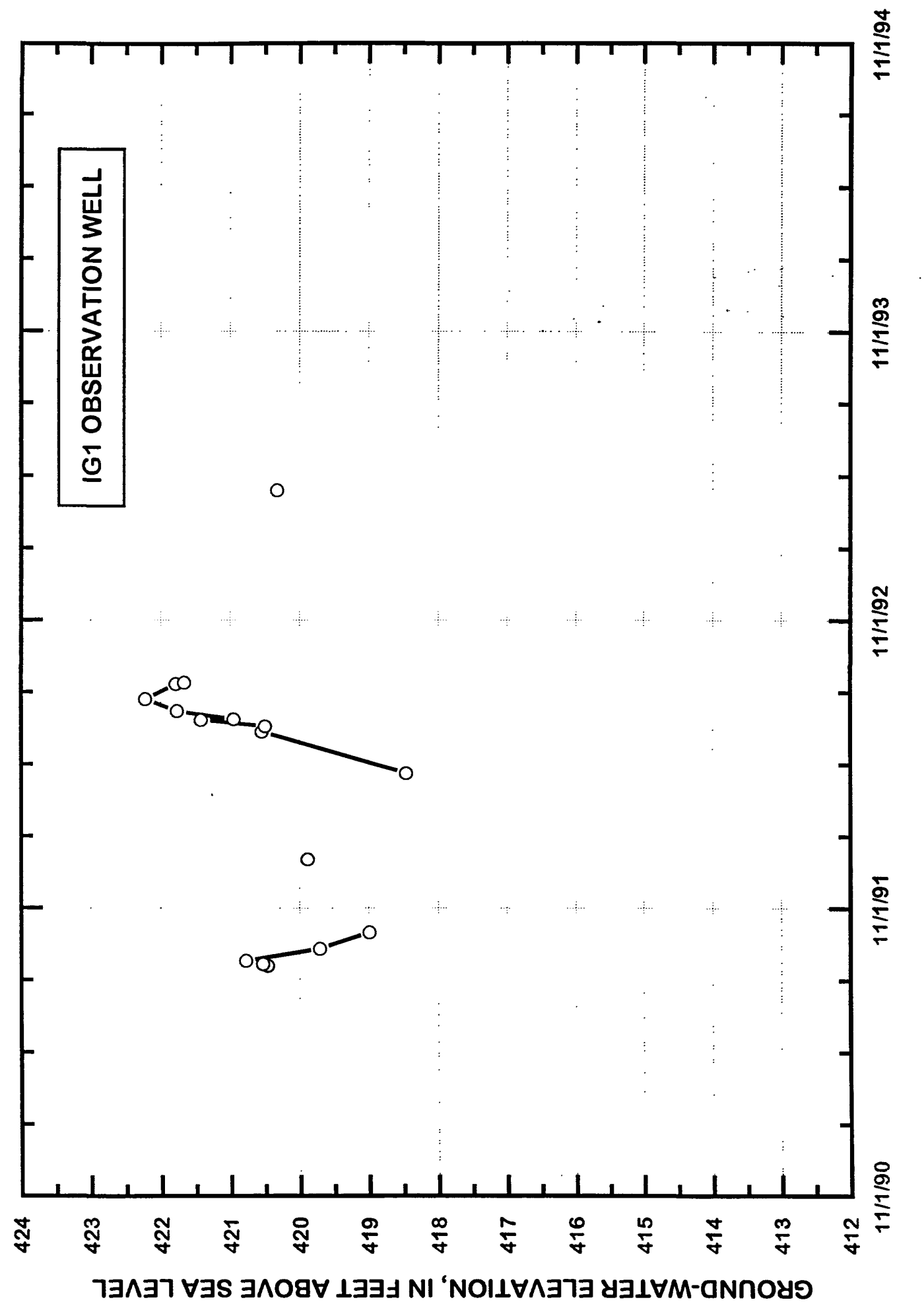



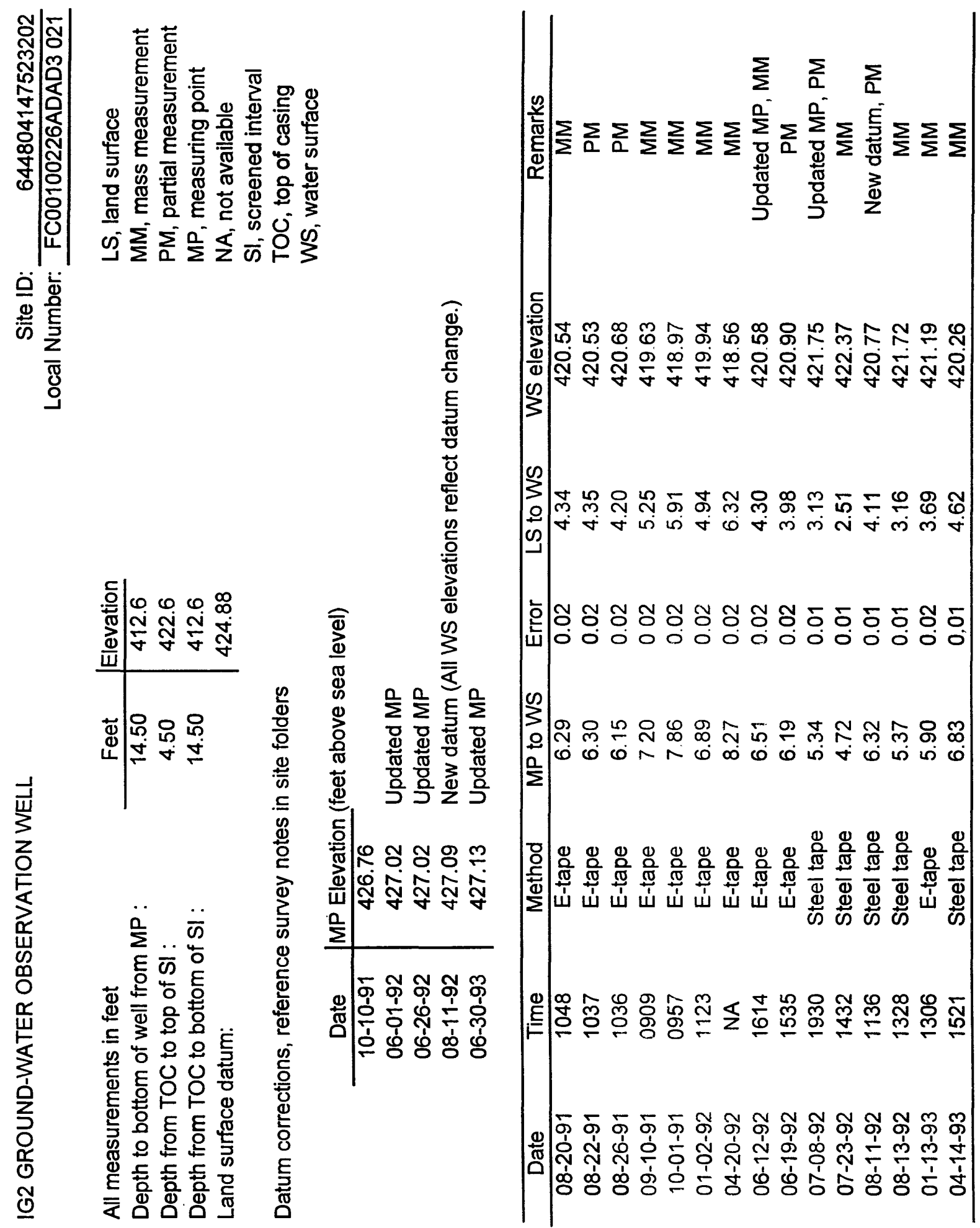


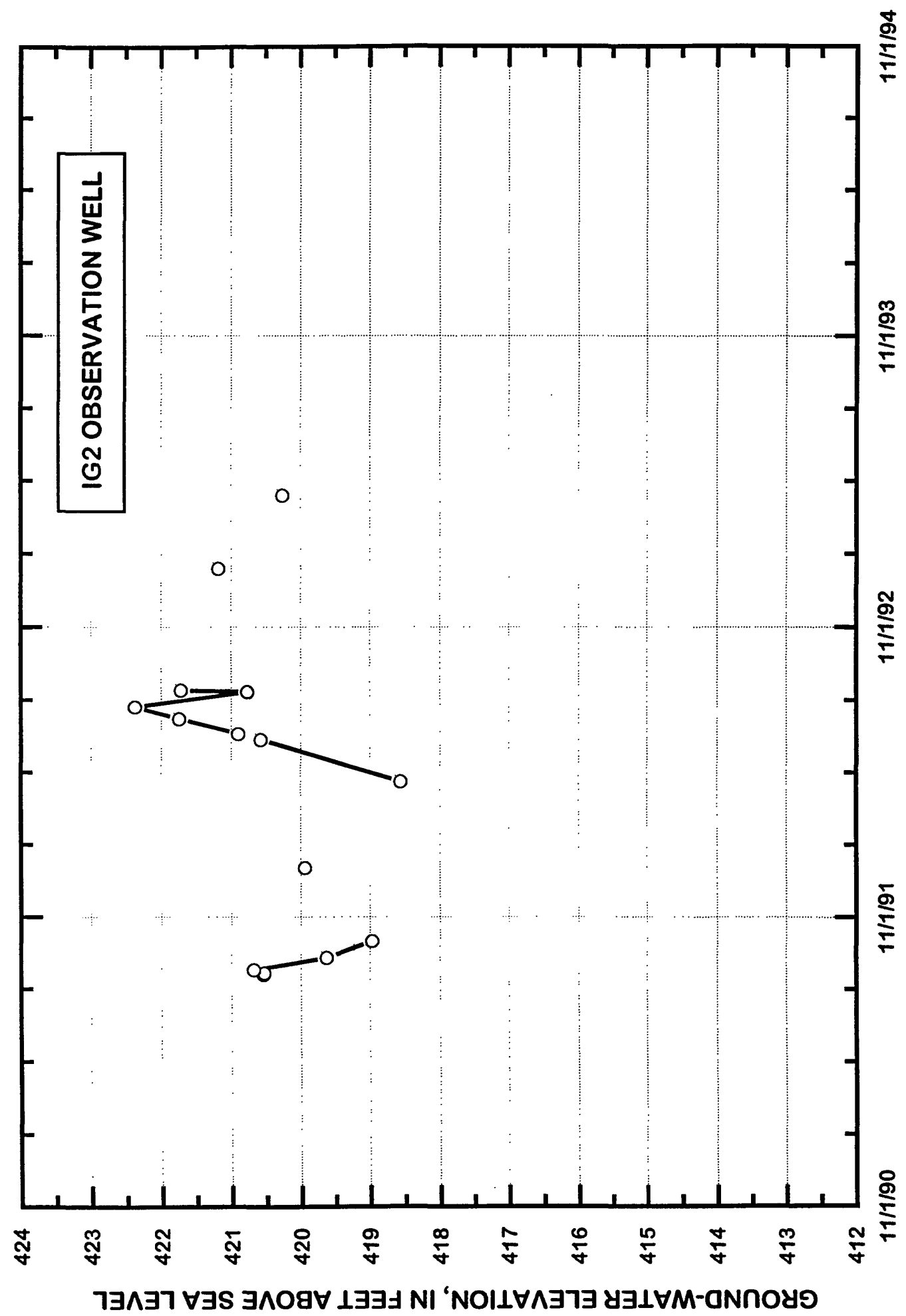

WATER-ELEVATION TABLES AND HYDROGRAPHS 67 

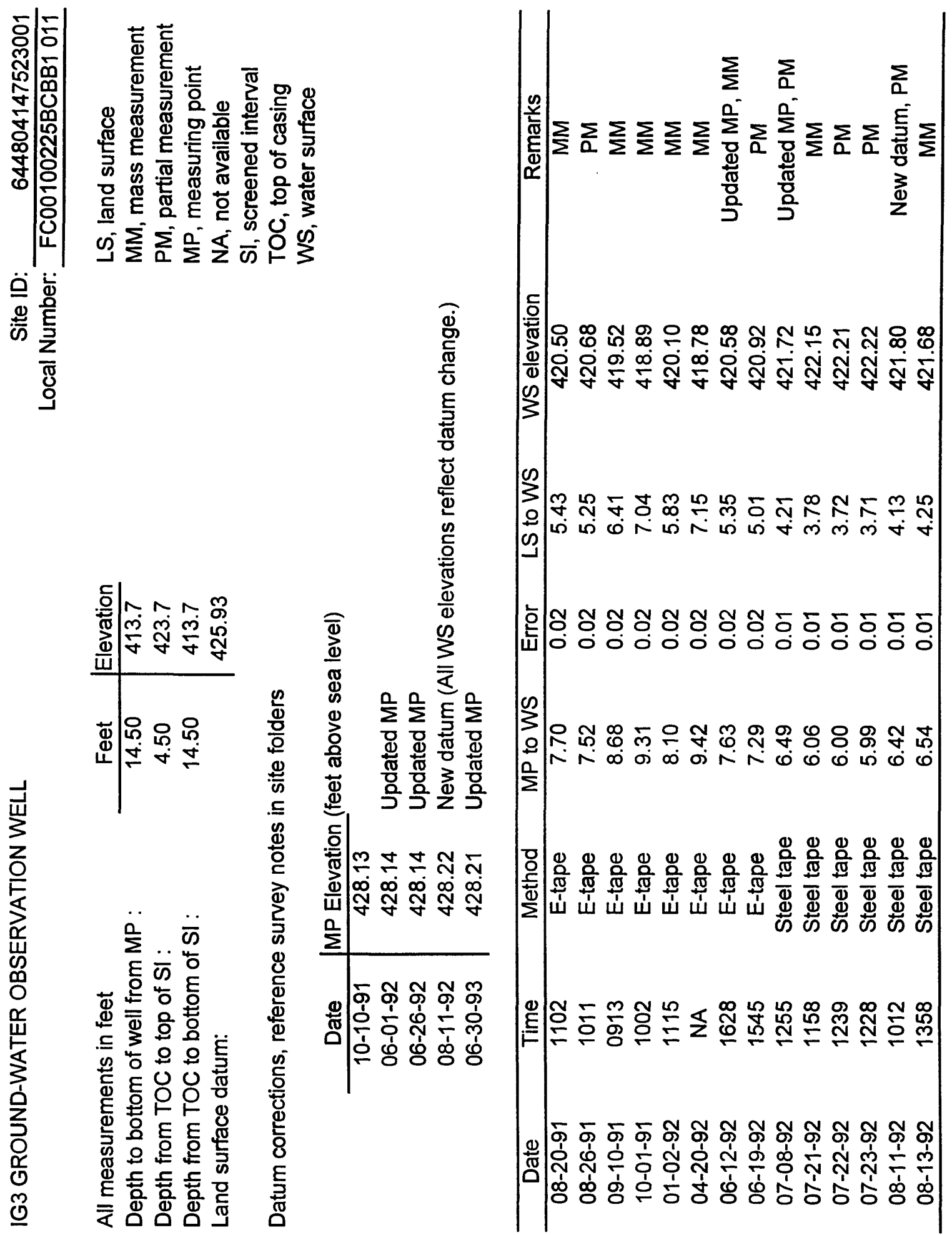

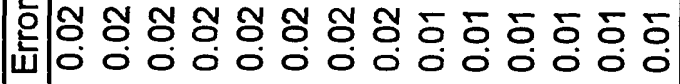

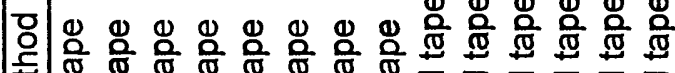

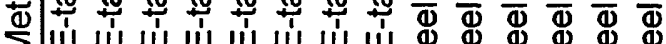

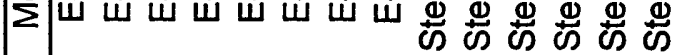

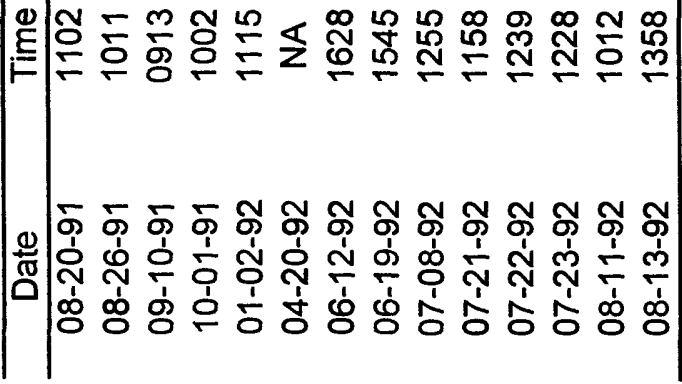




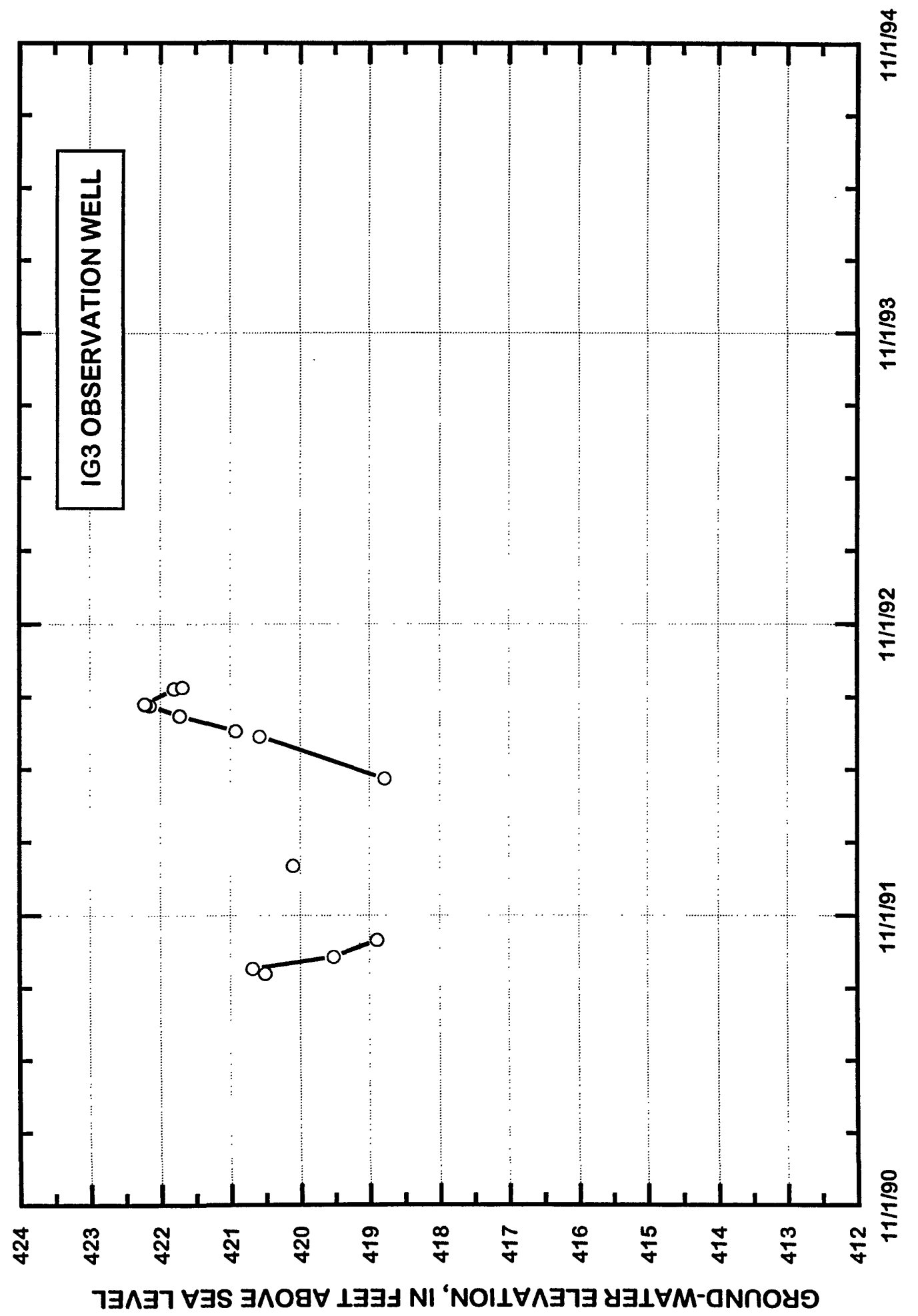



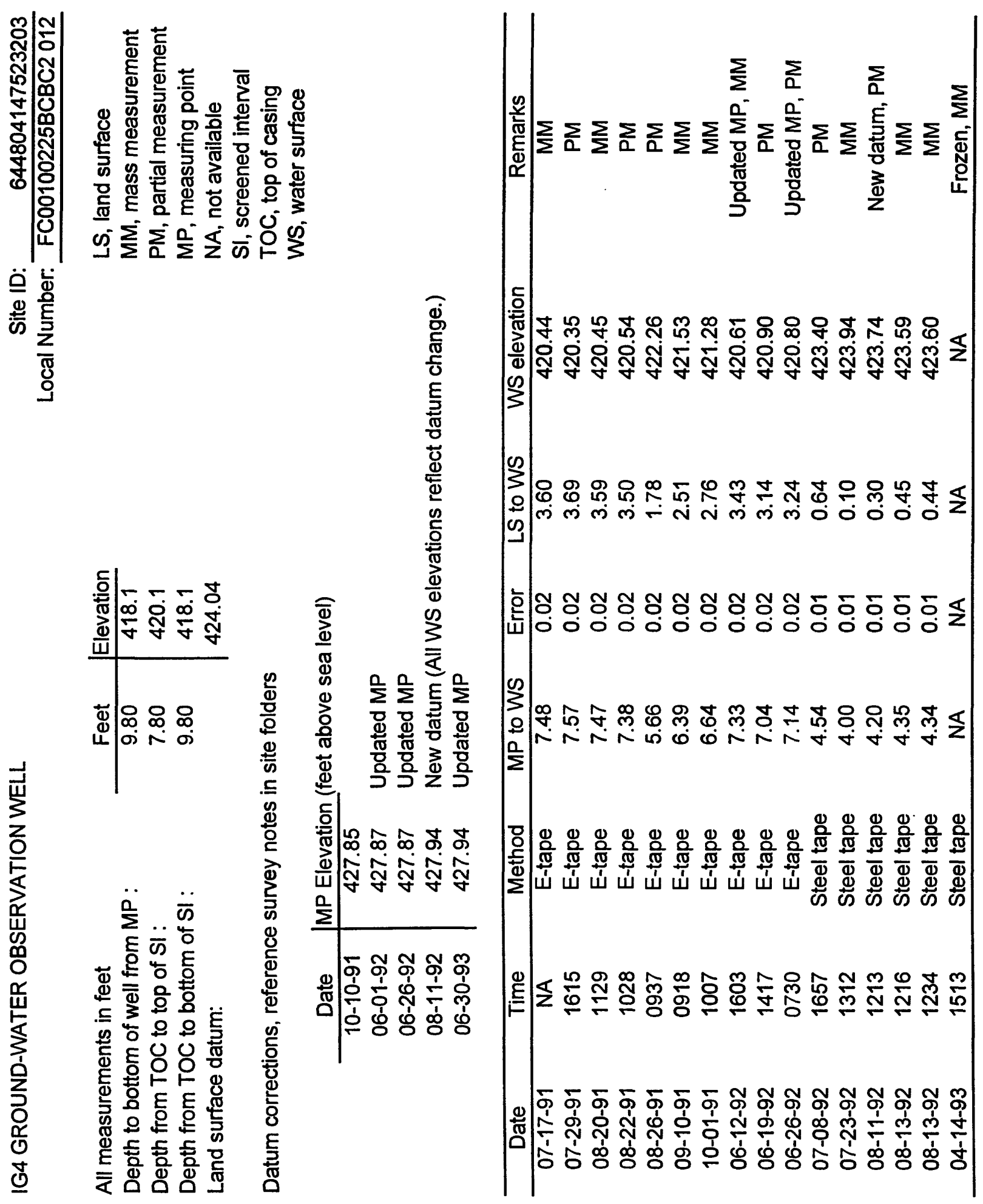


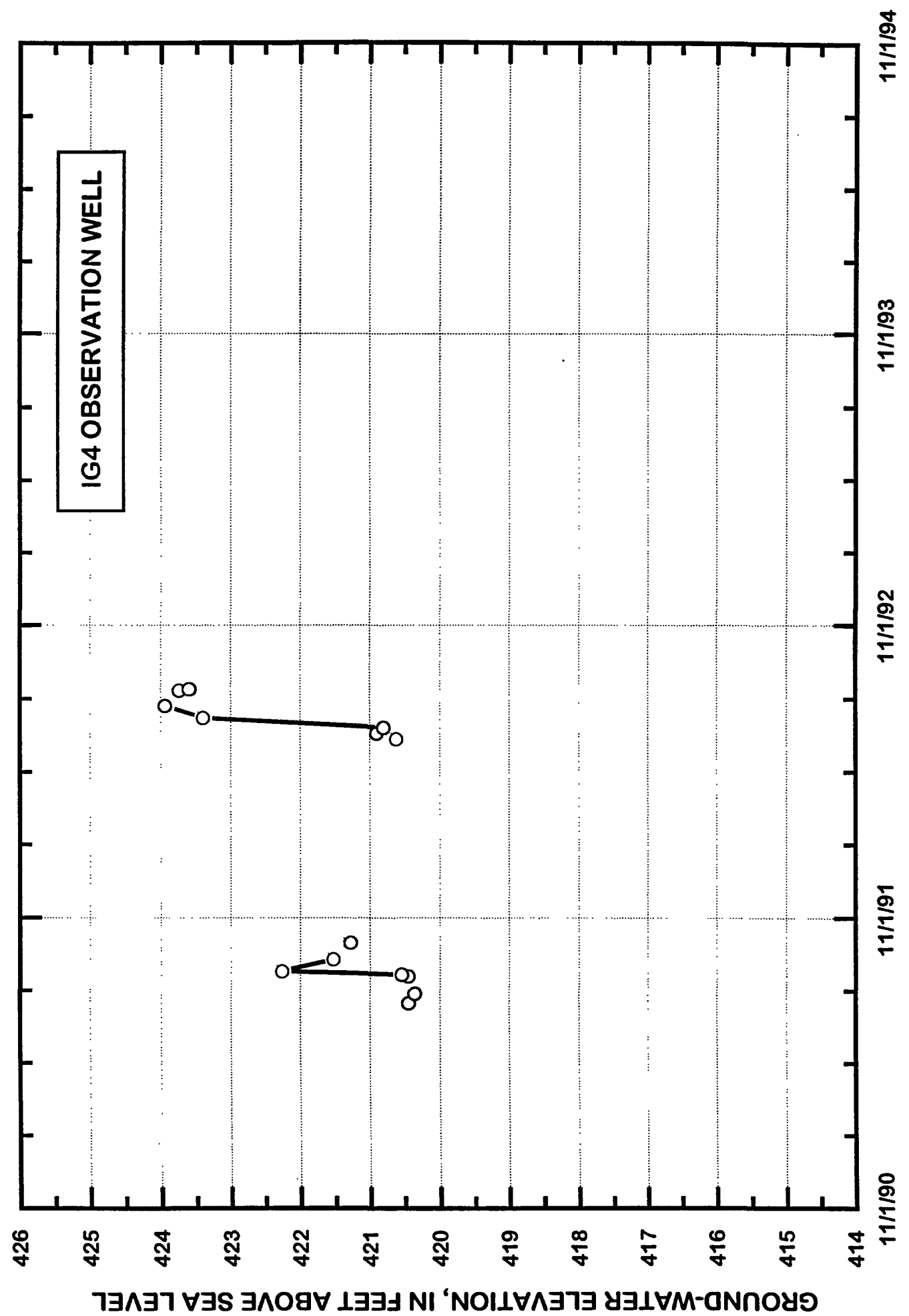

WATER-ELEVATION TABLES AND HYDROGRAPHS 71 

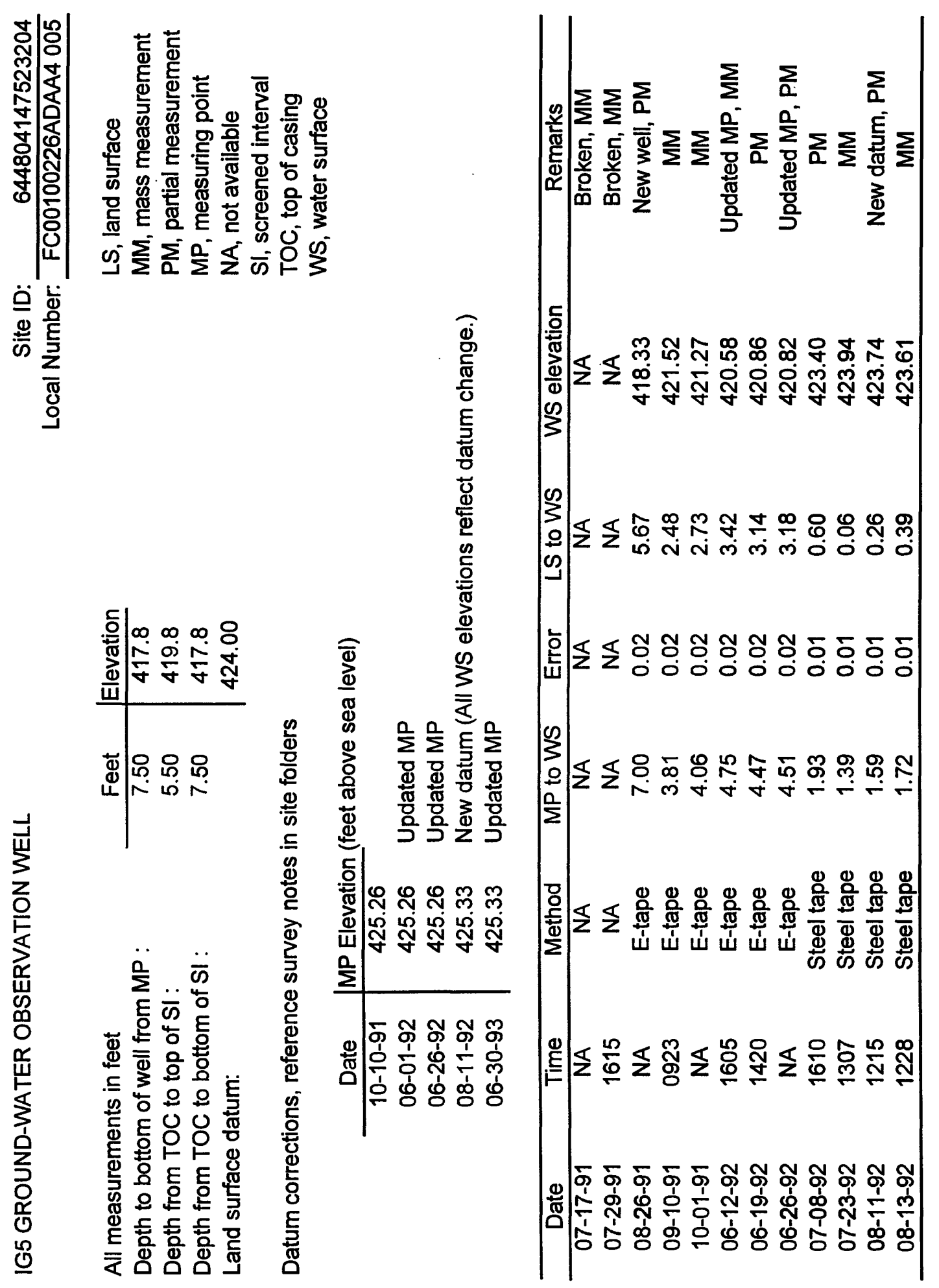


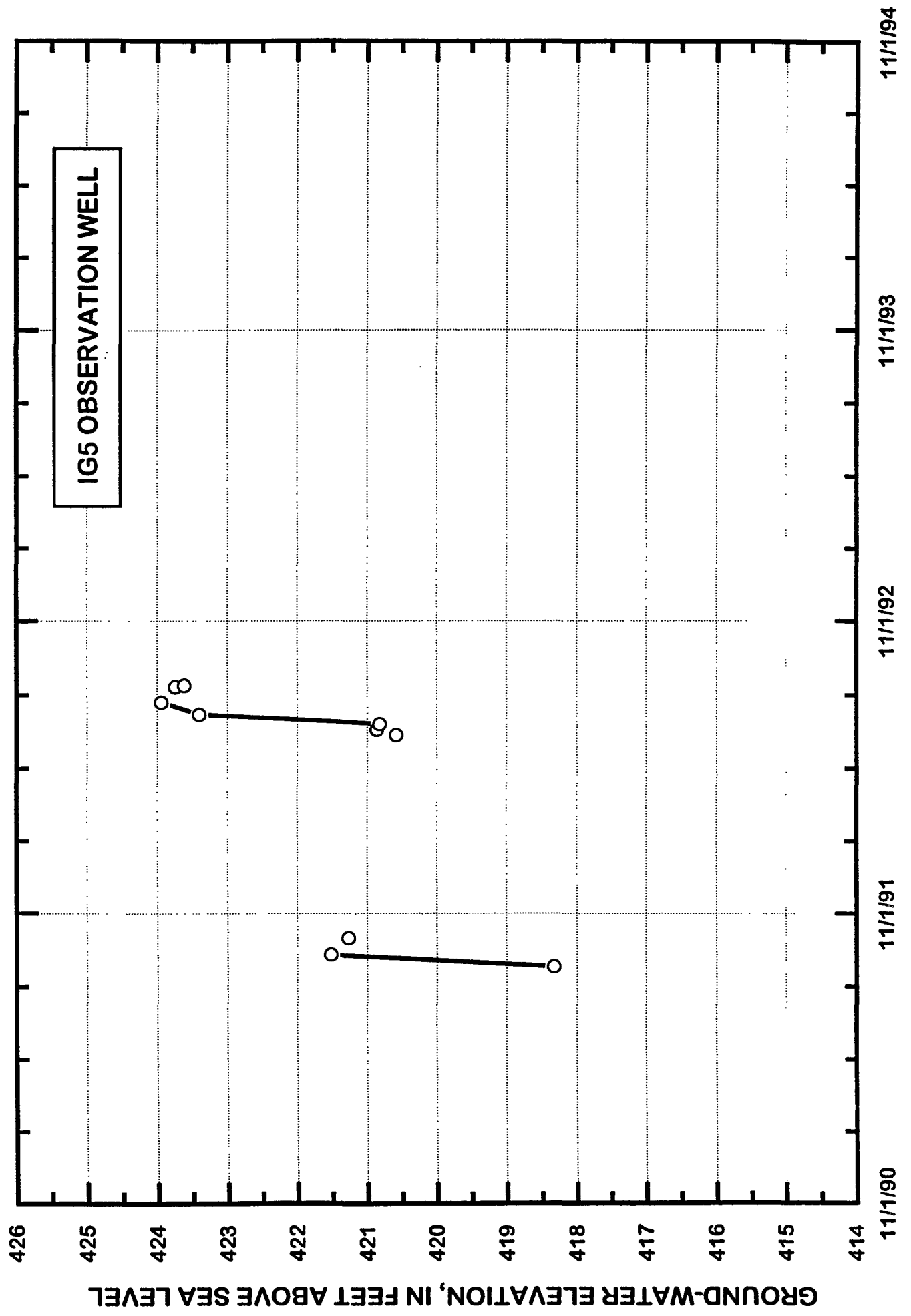



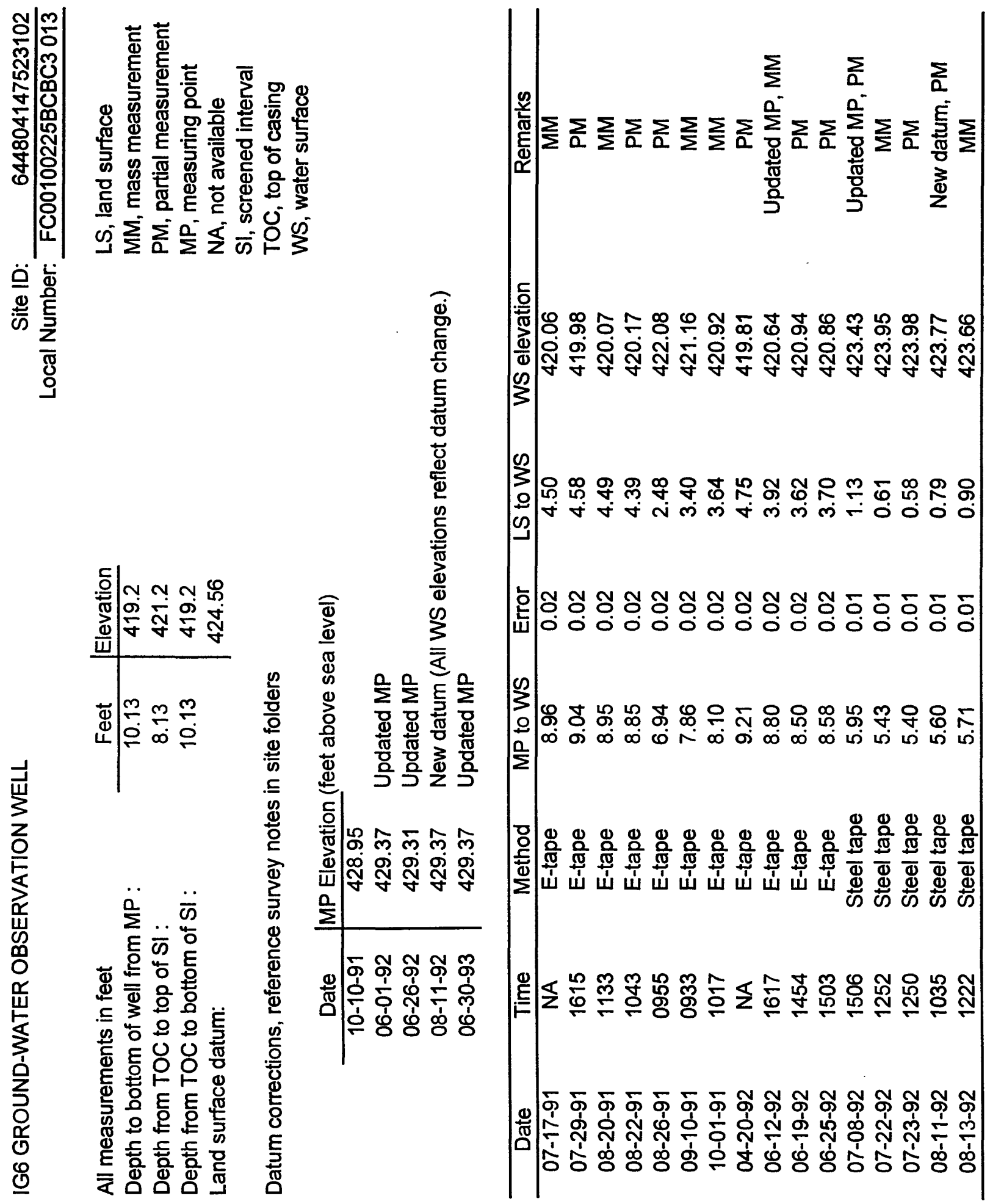


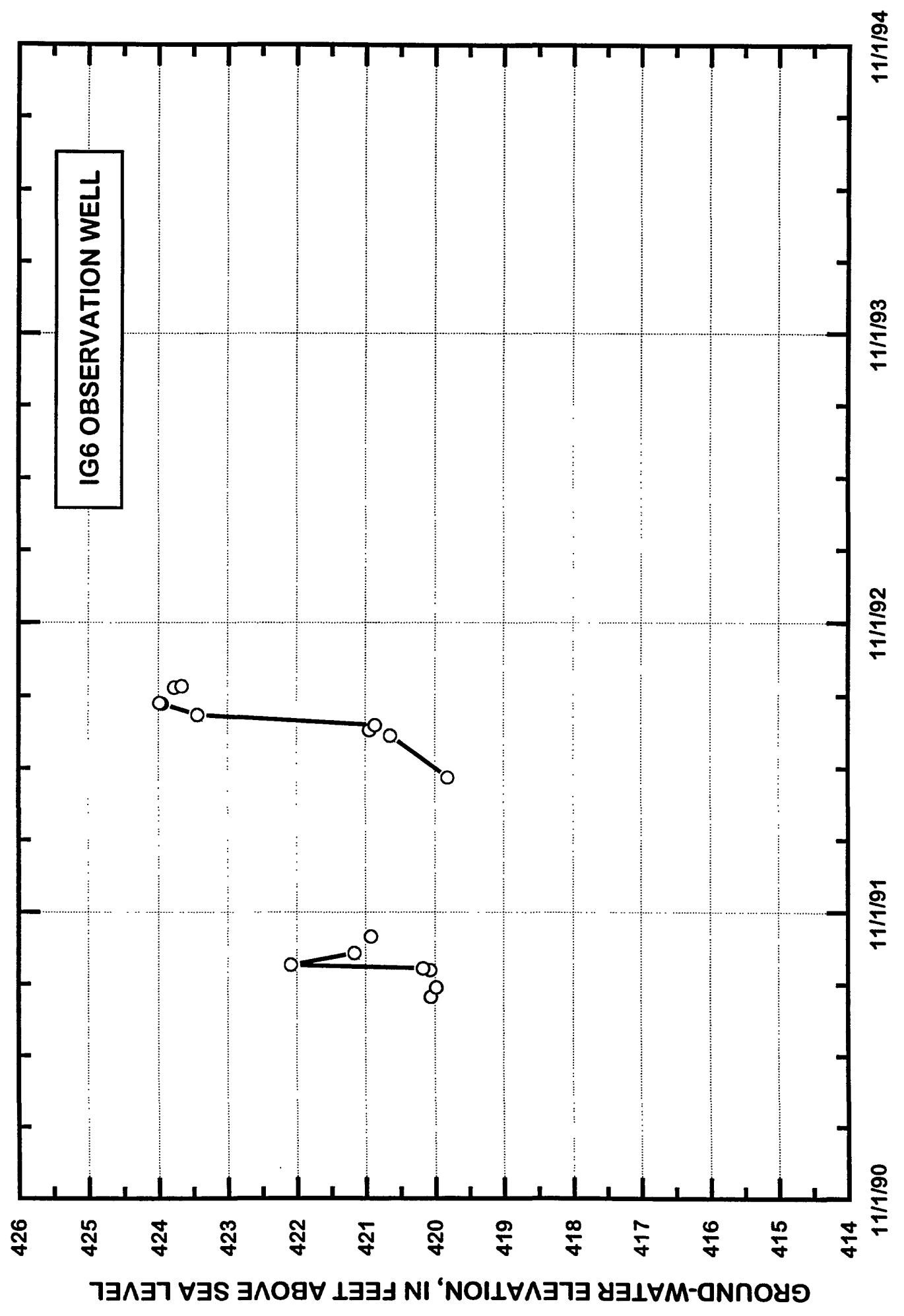




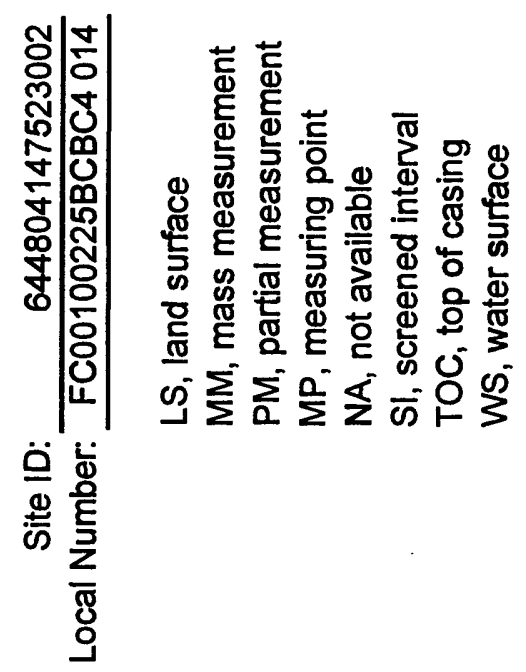

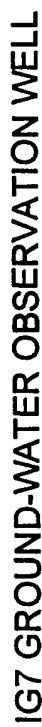

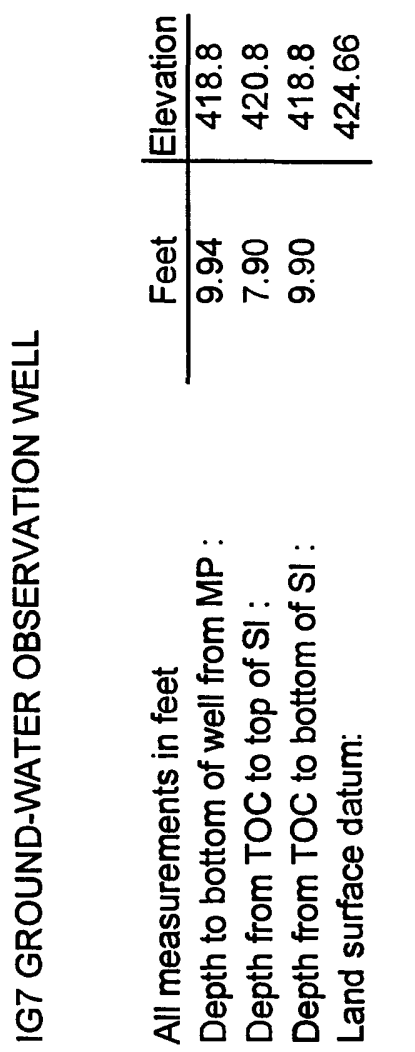

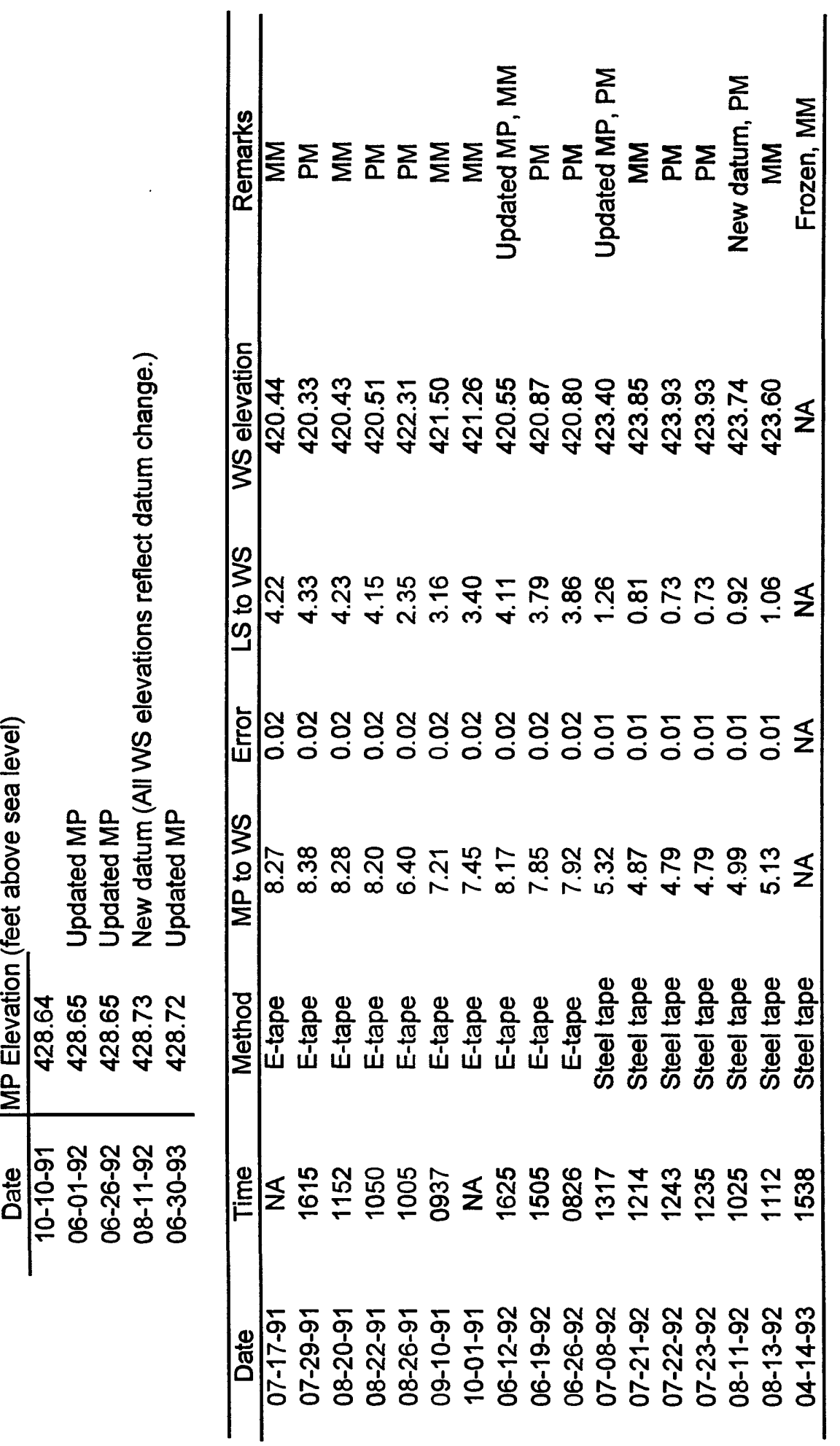




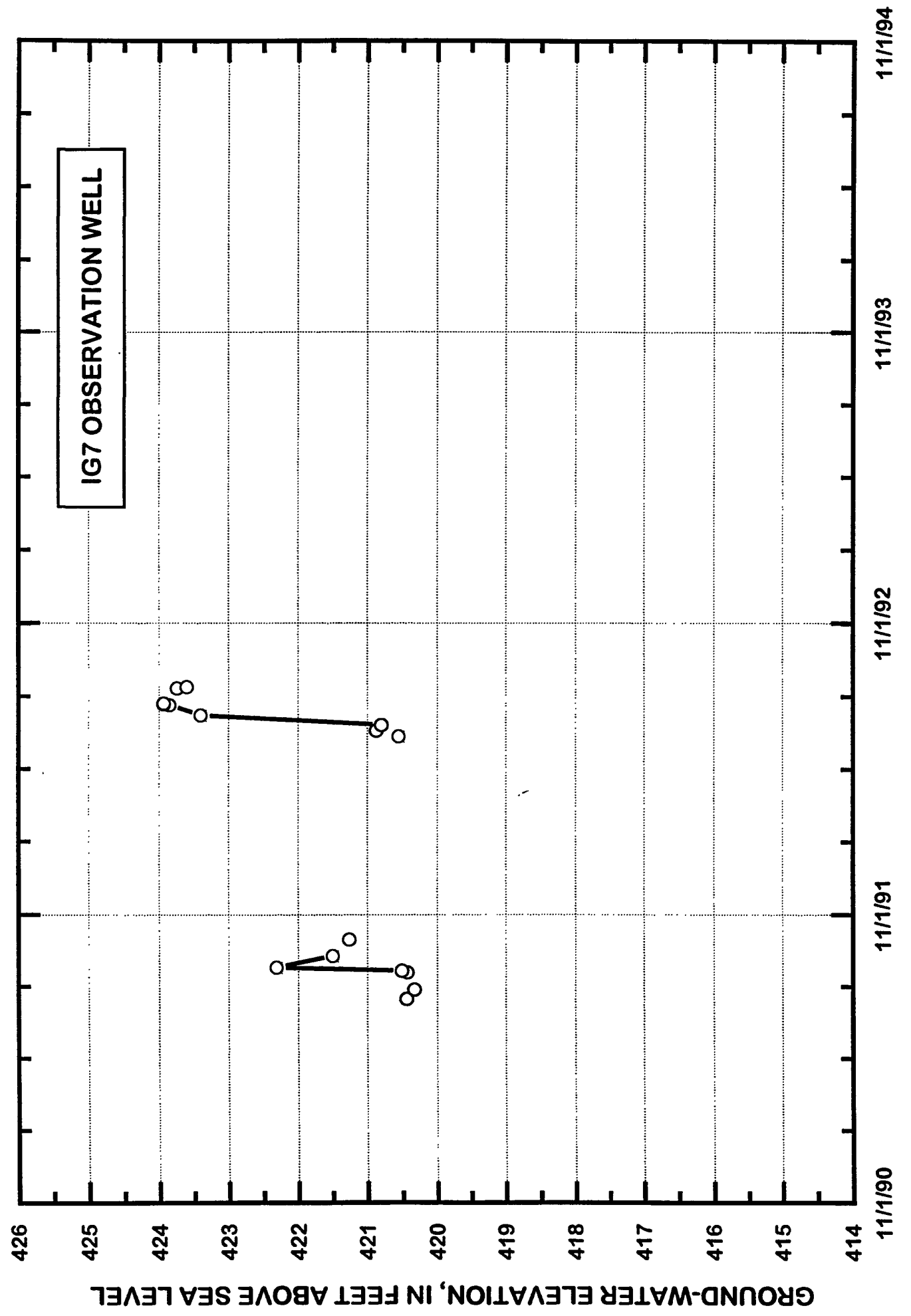



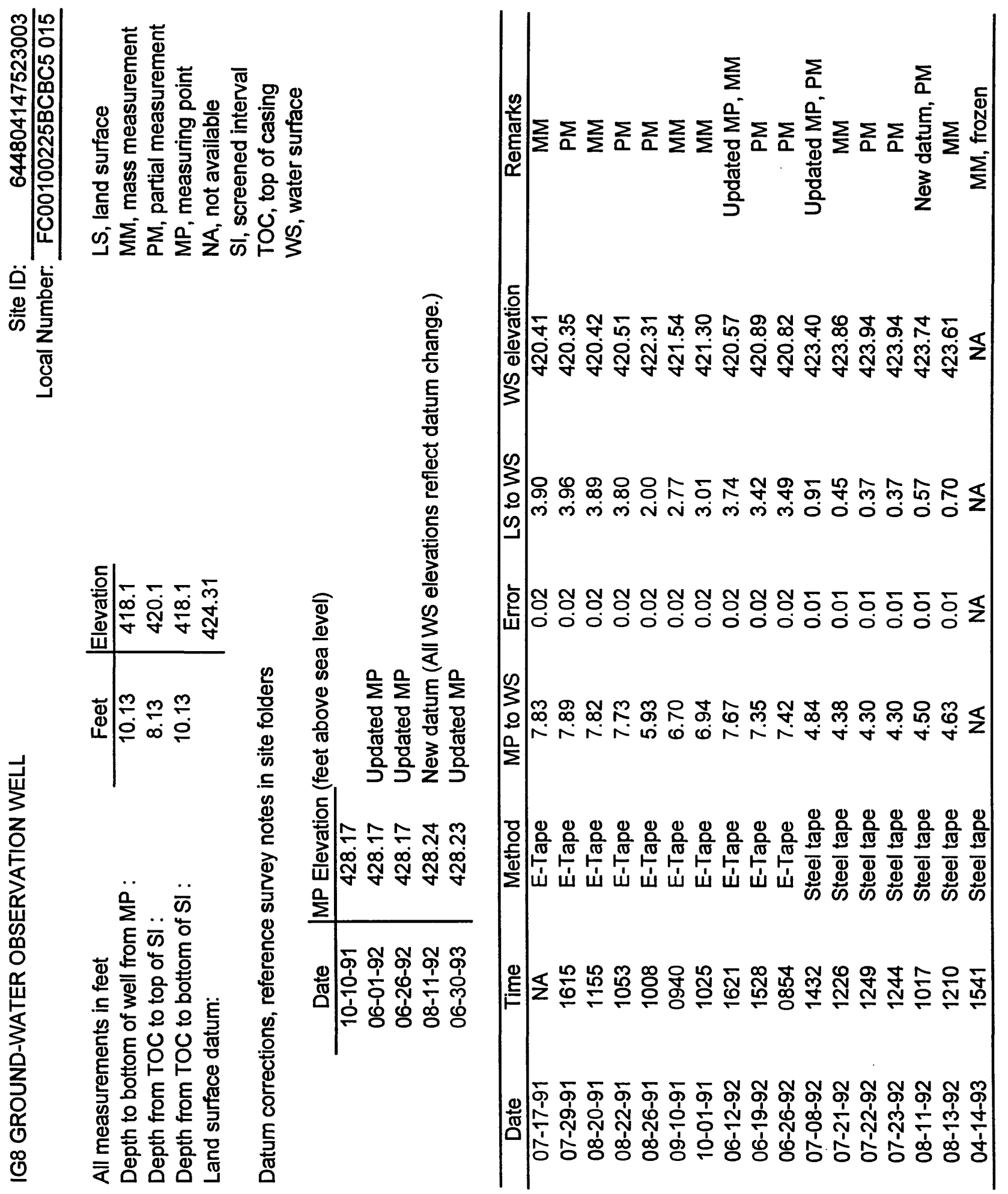


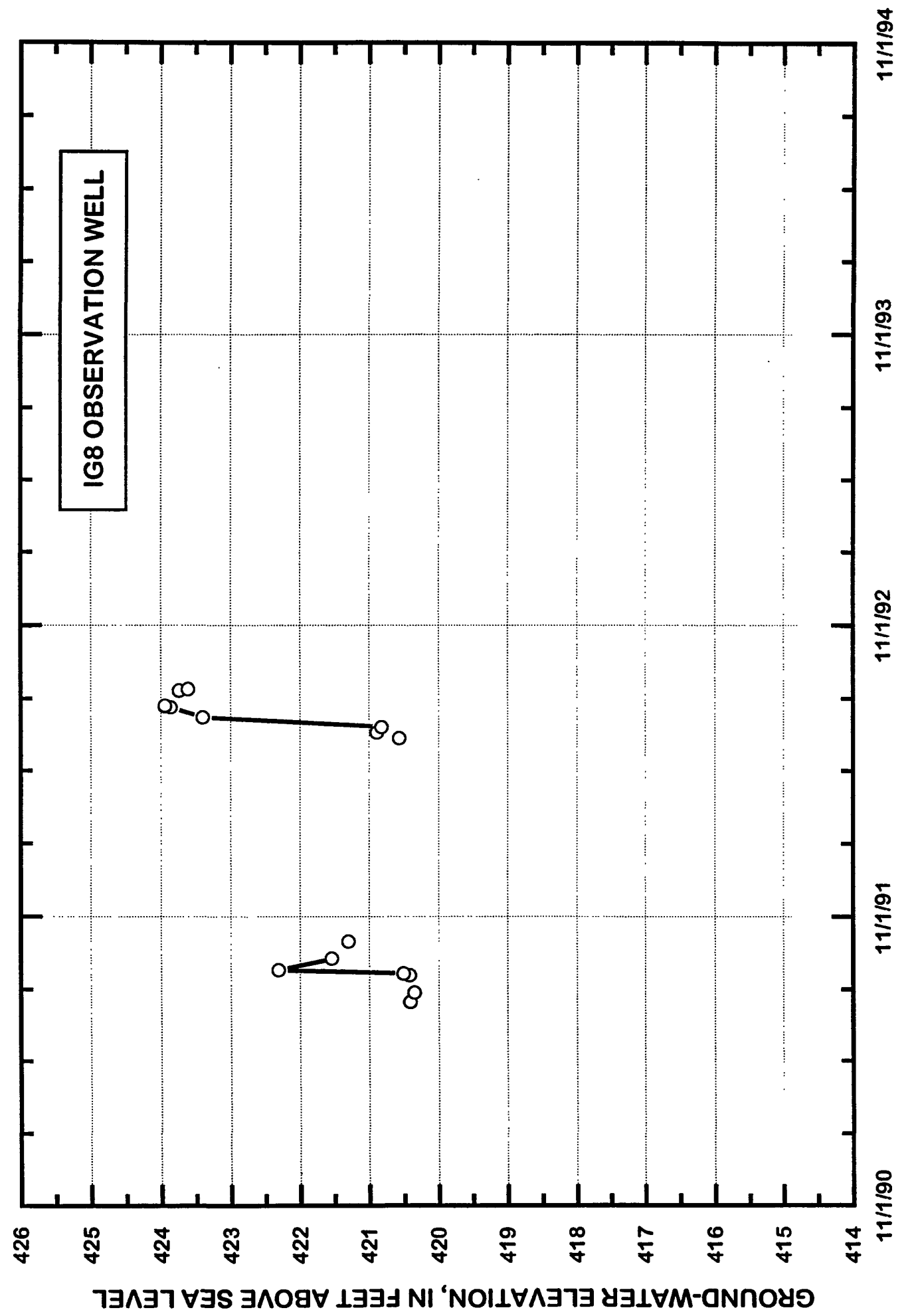



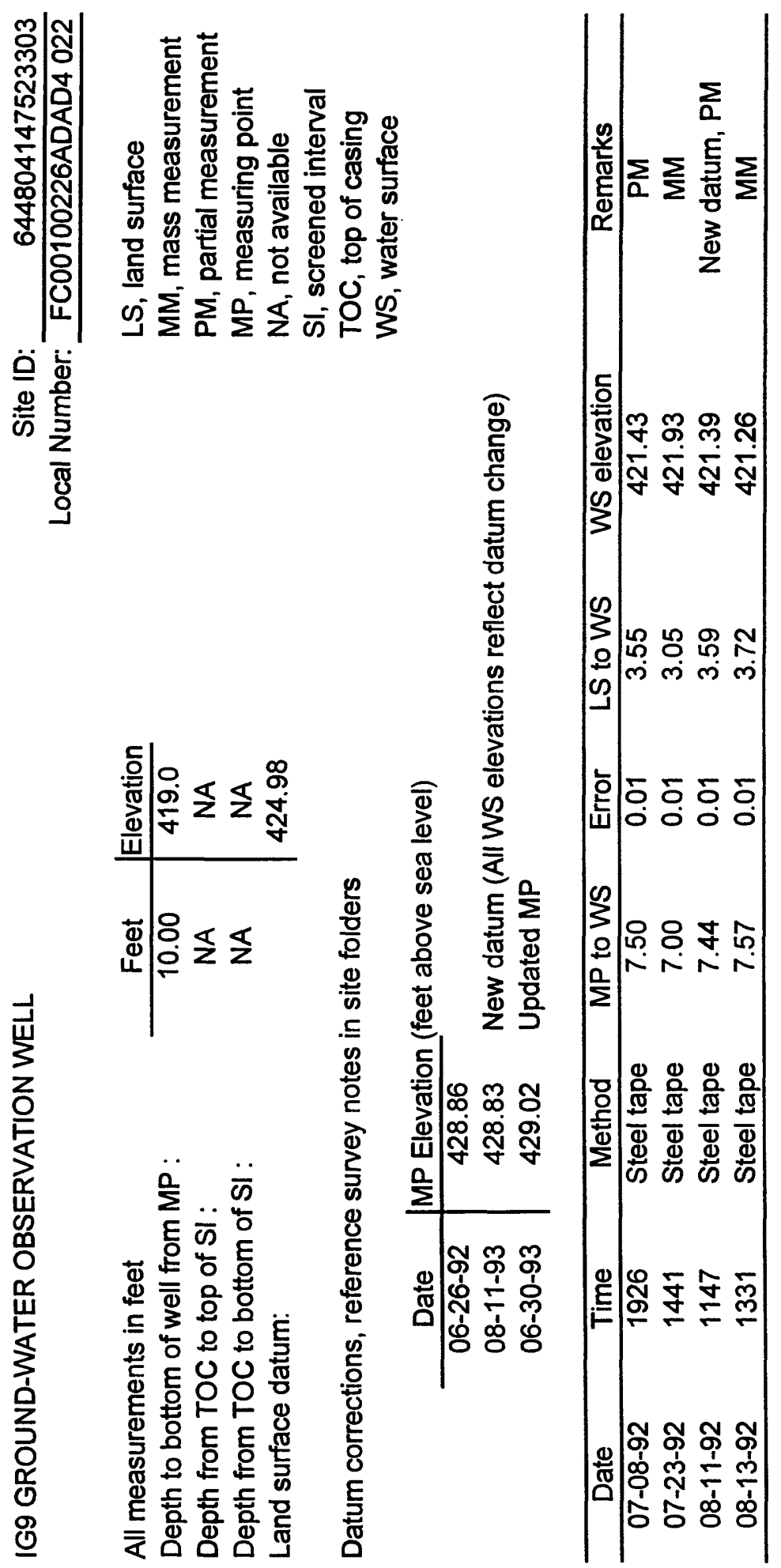


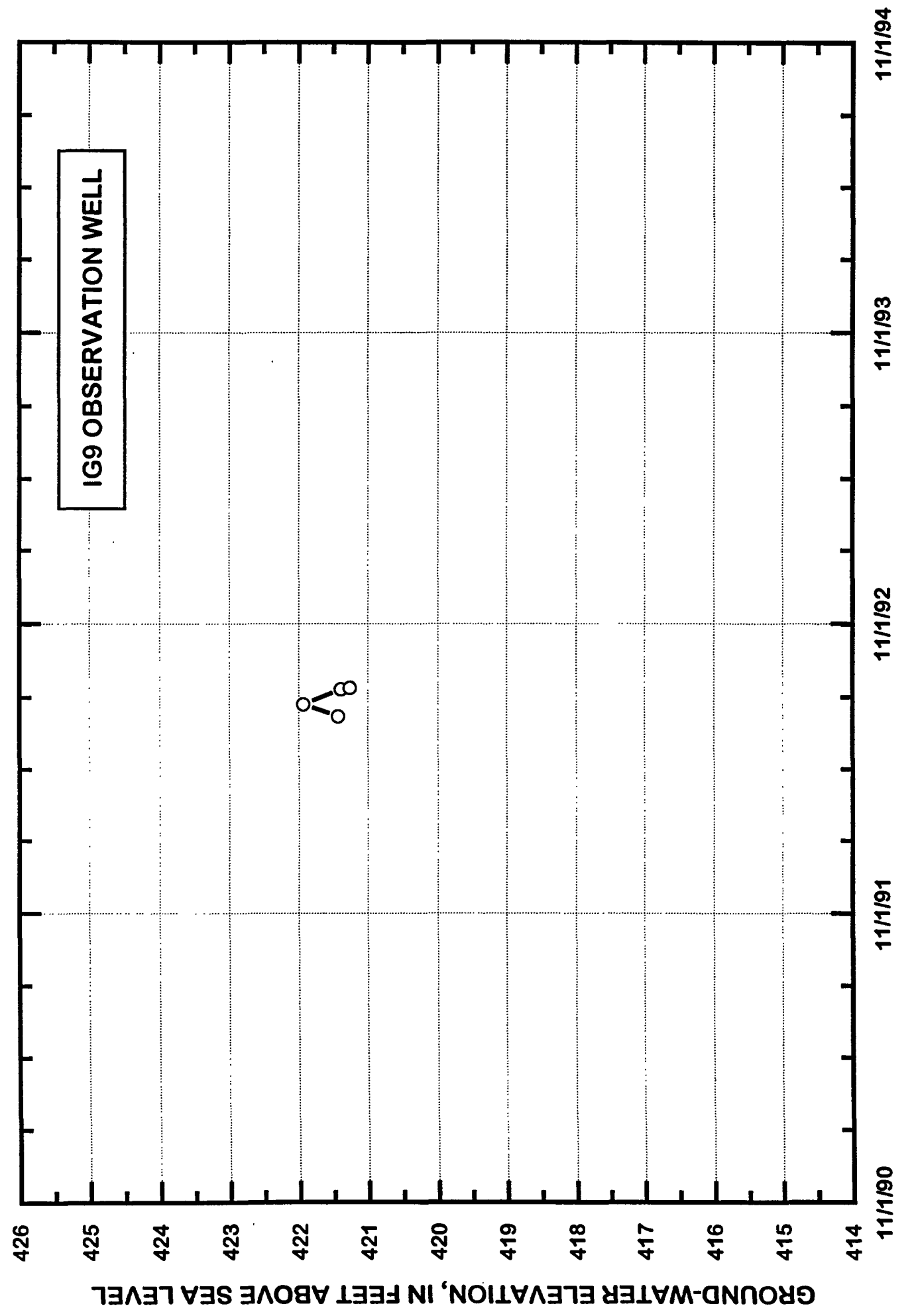



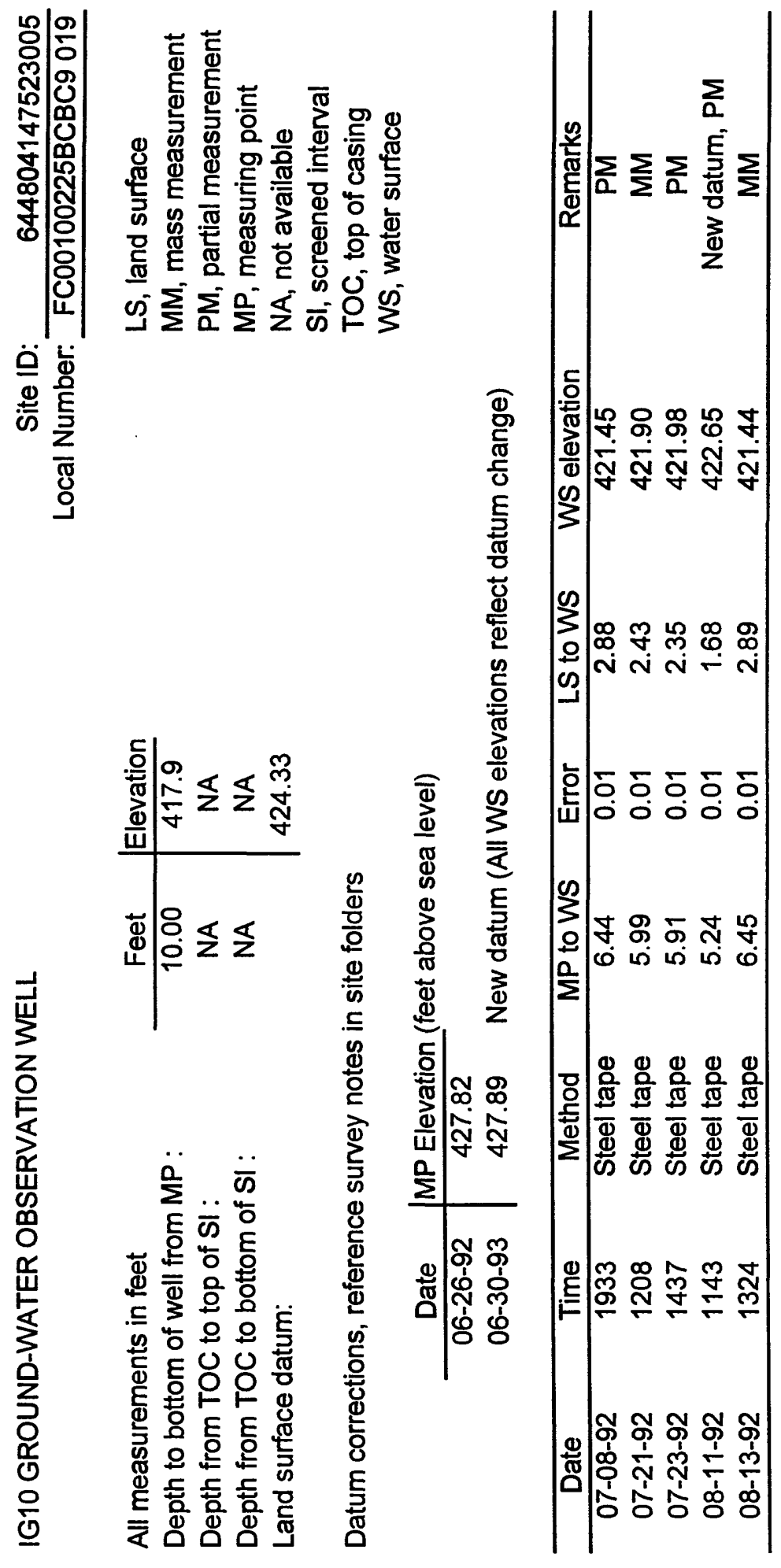


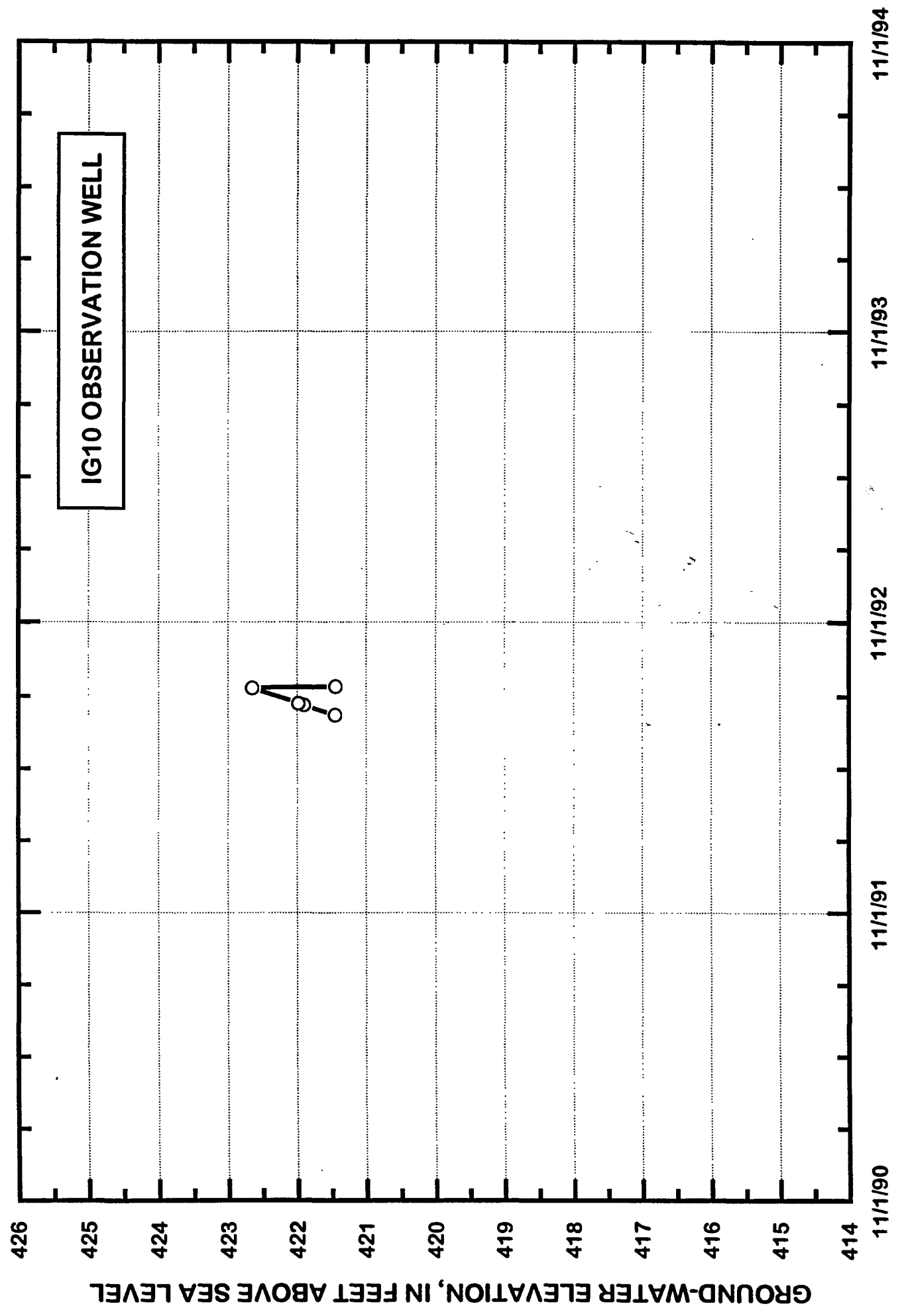



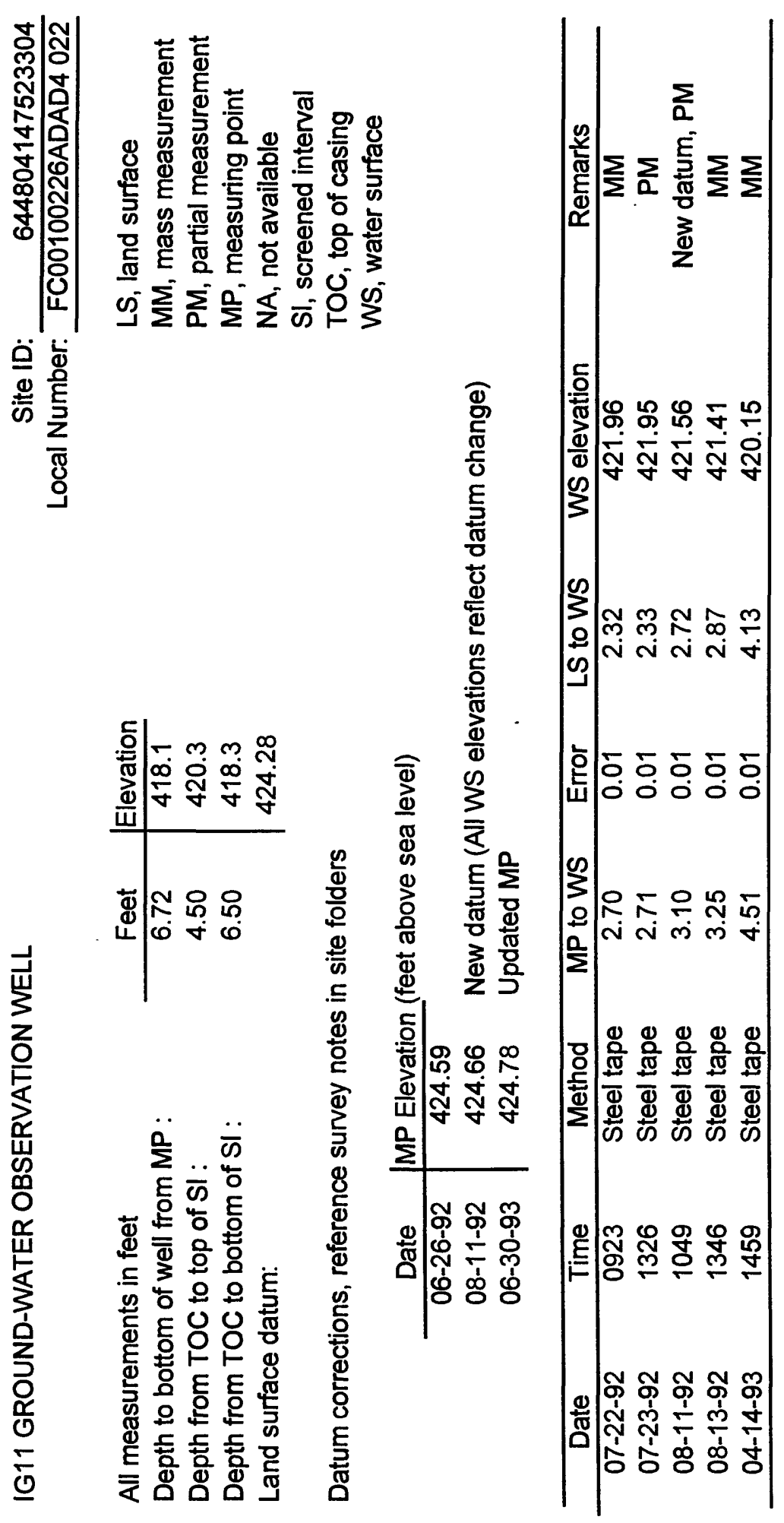


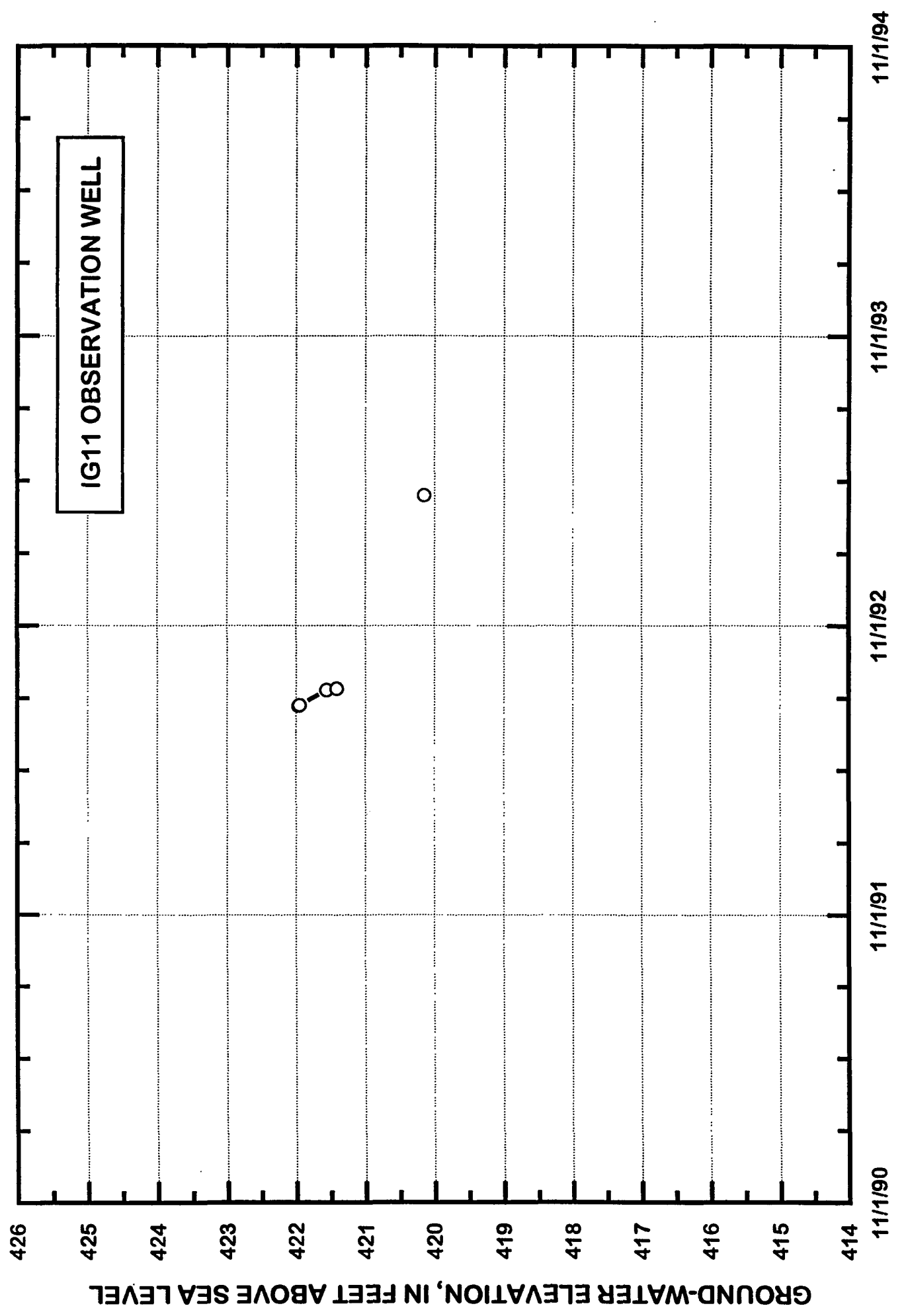



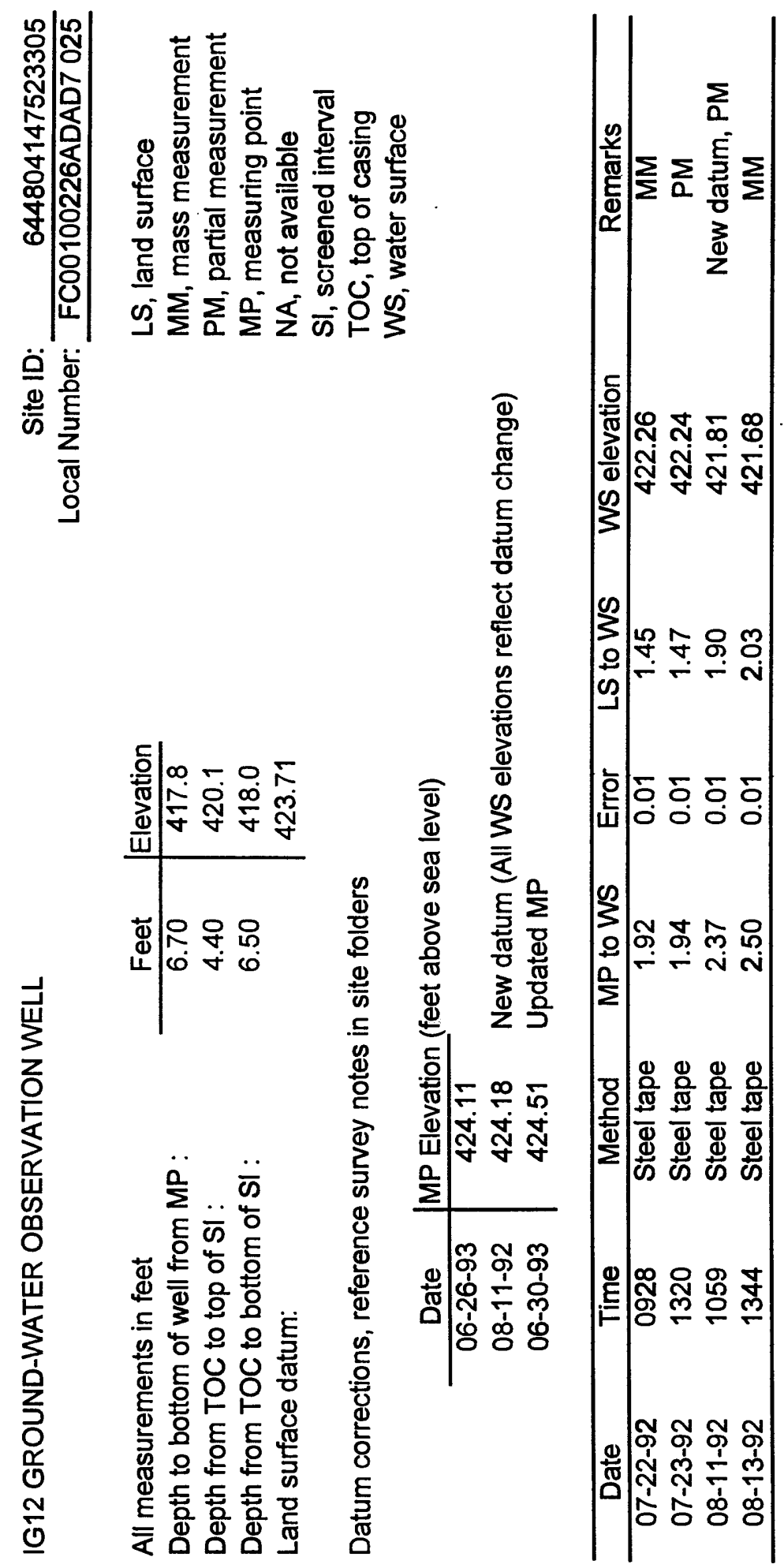


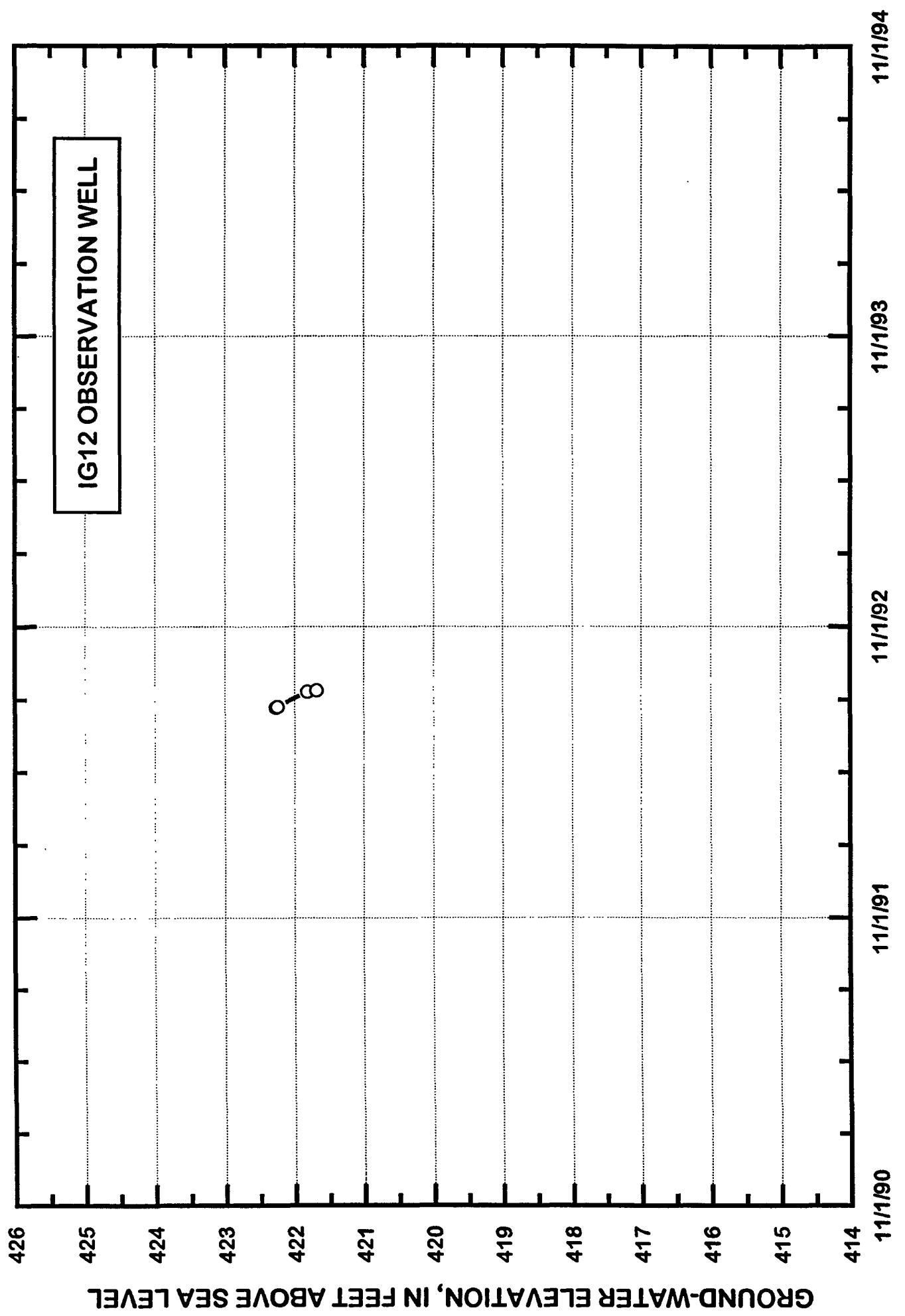



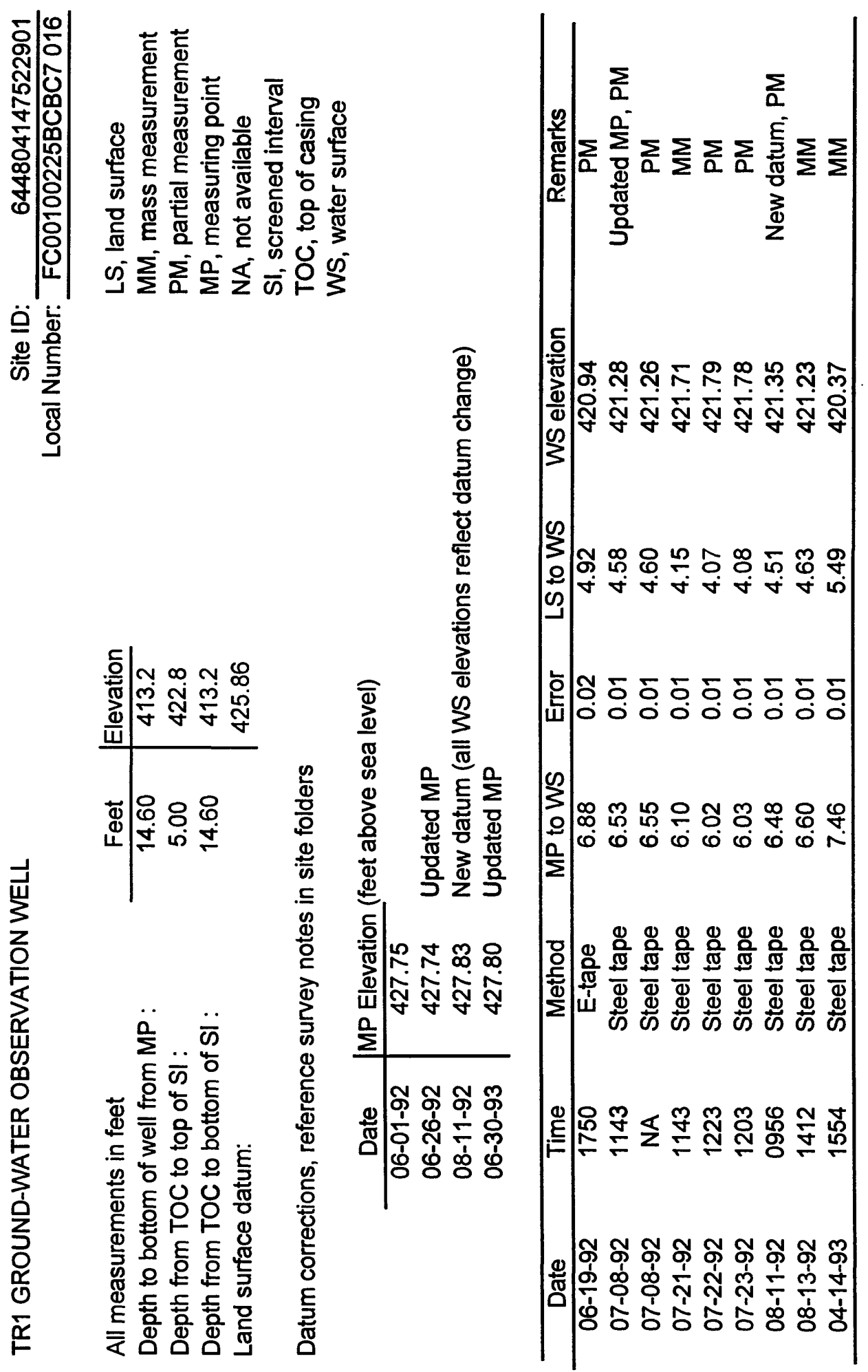


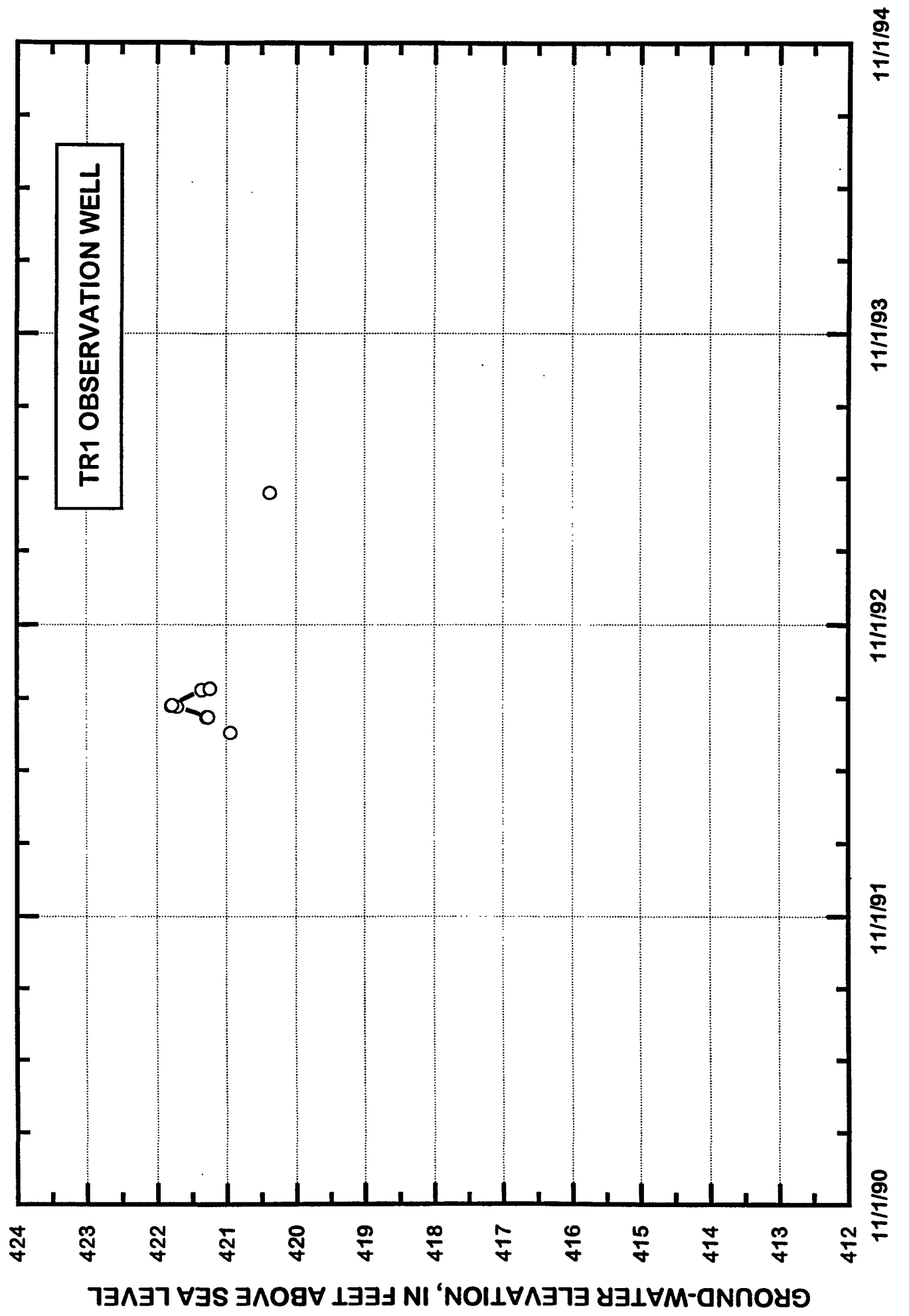



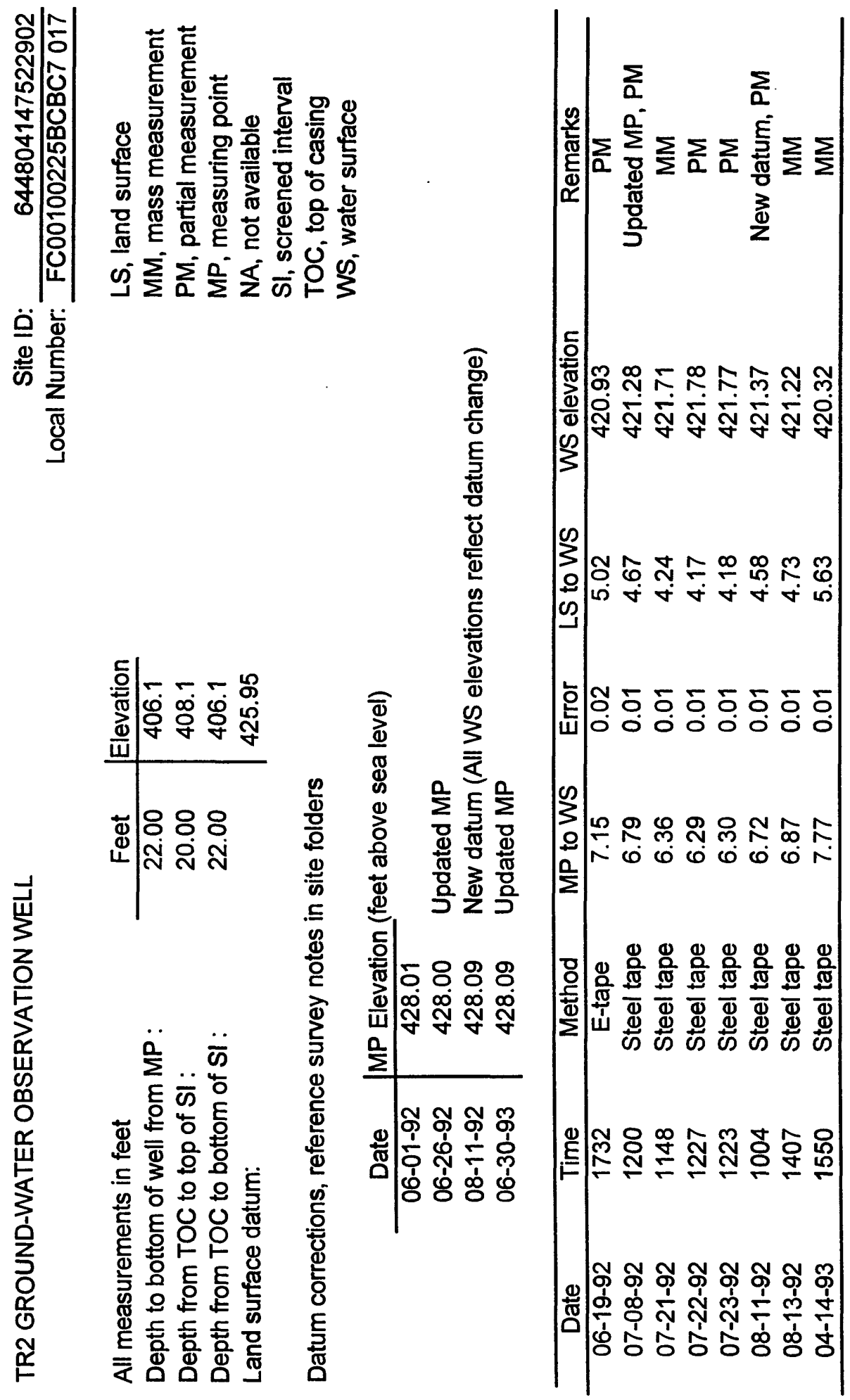


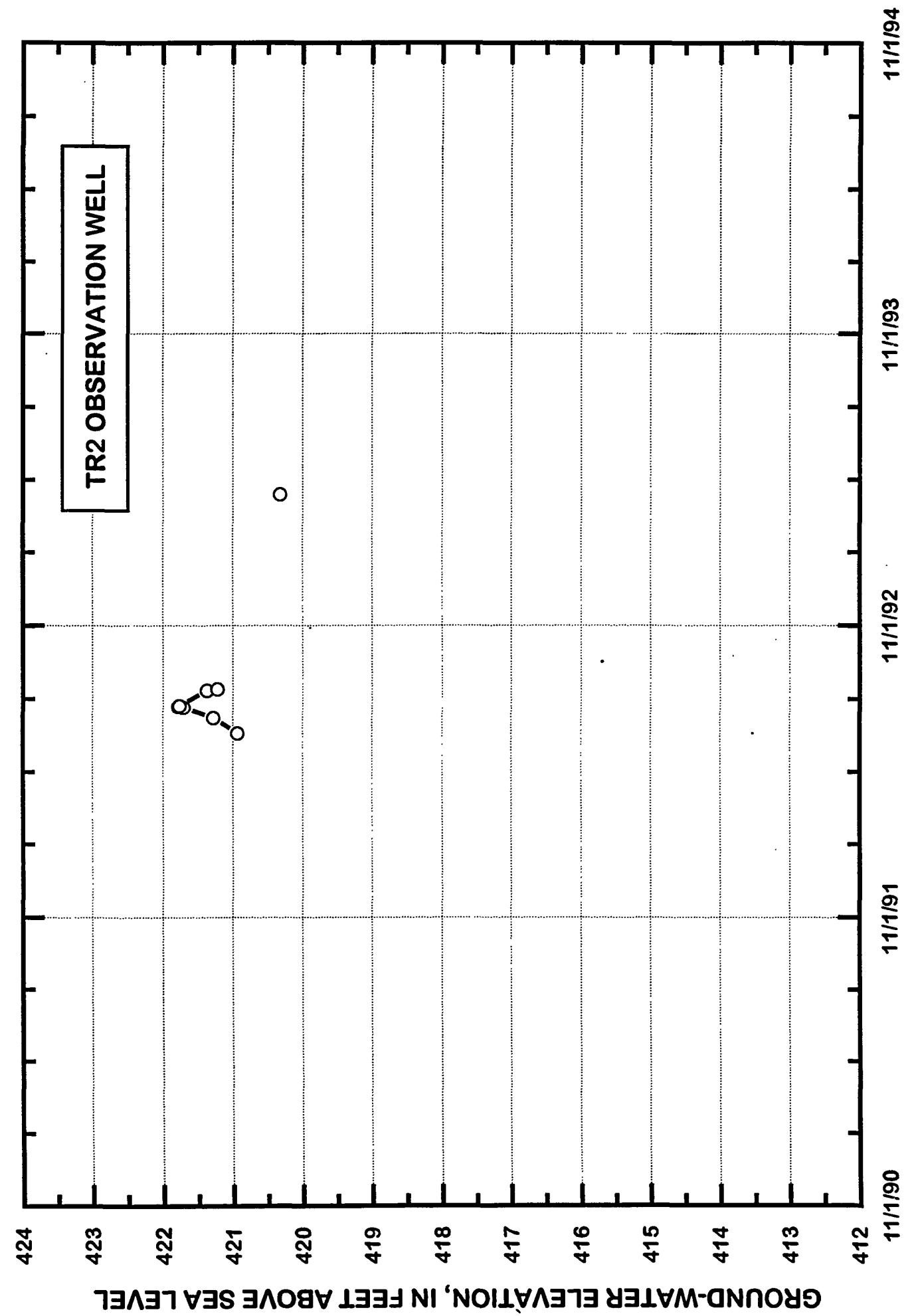



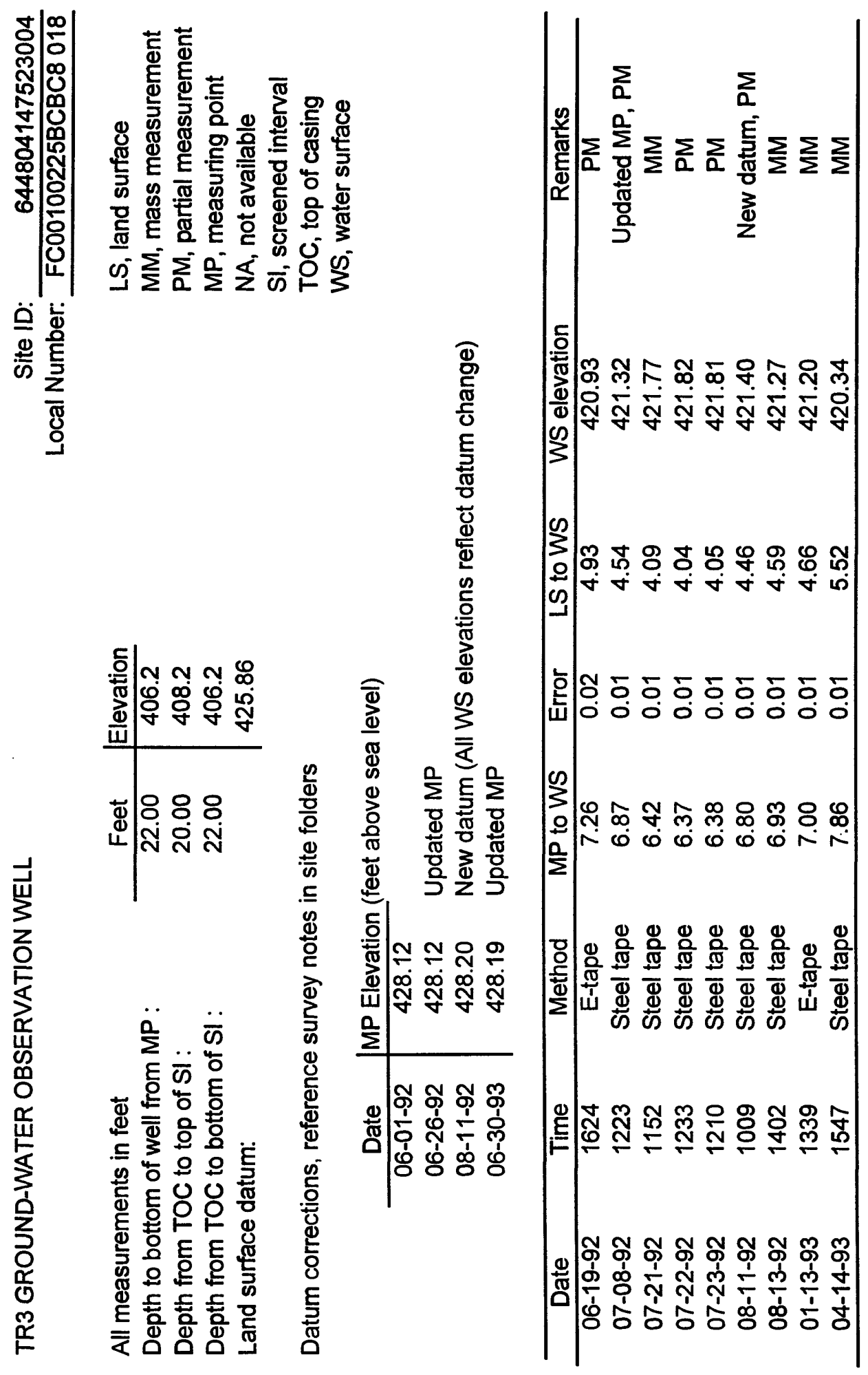


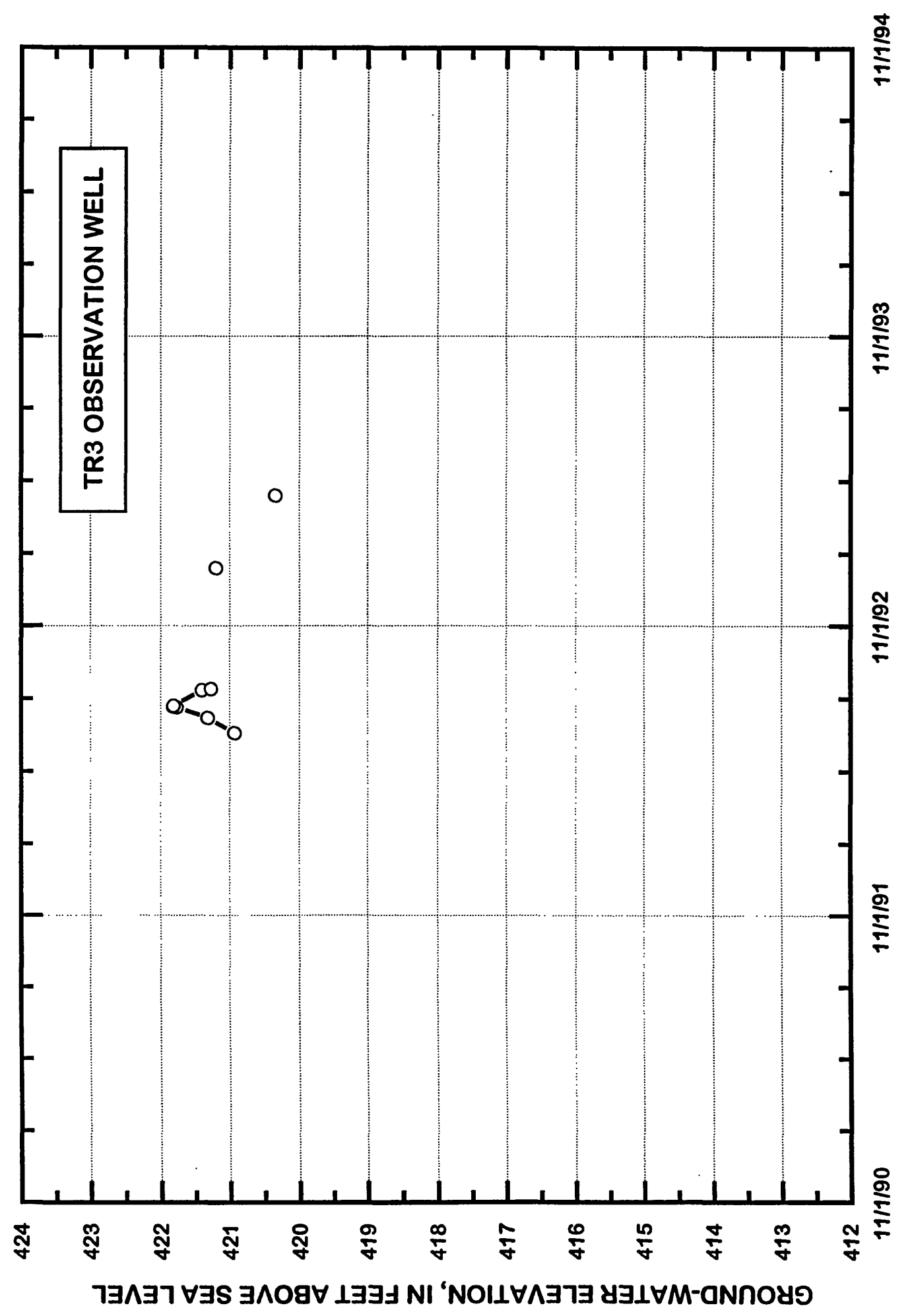




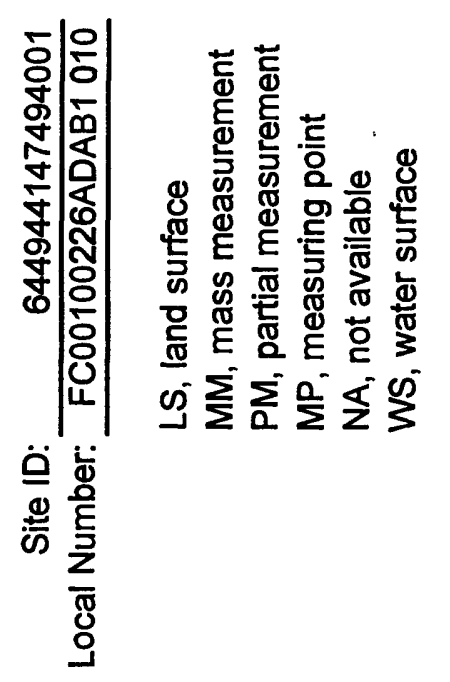

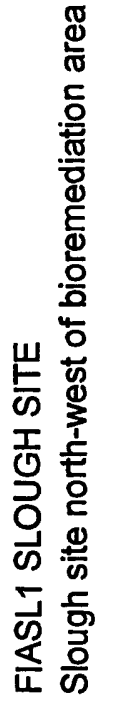
$\underset{\forall}{\infty}$

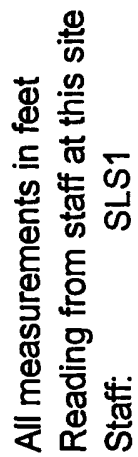

$\mid$

ฟัษ 守 守 守

$\infty$

| co †

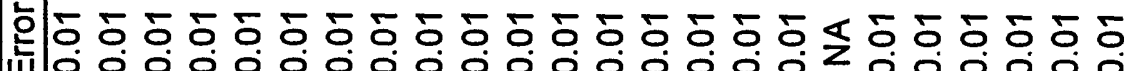

잉 \& 8 웅 号

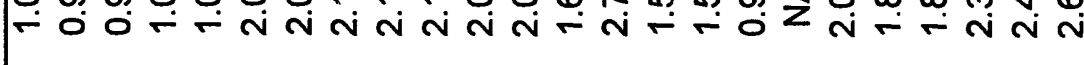

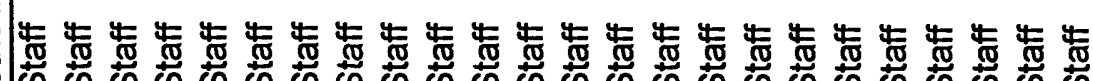

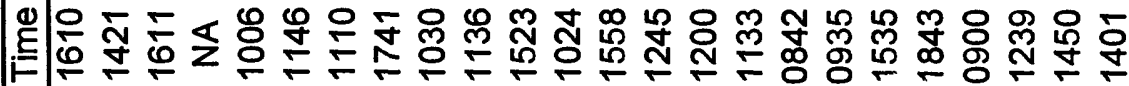

চ

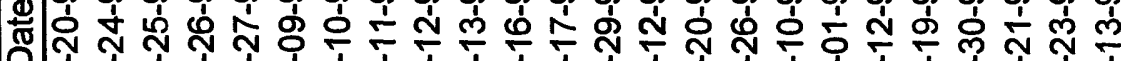

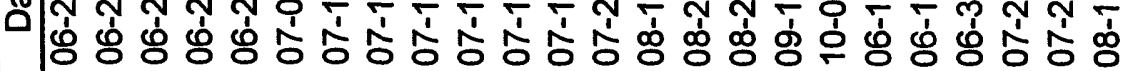




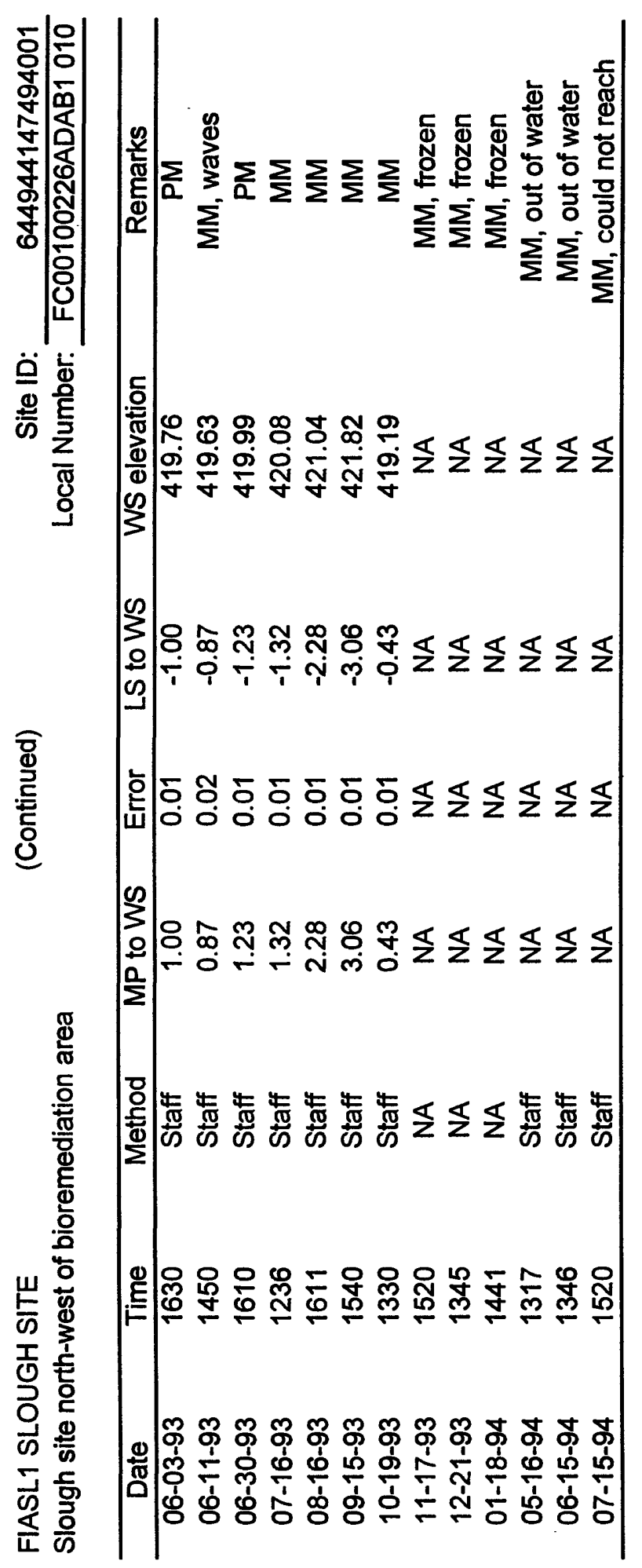


96 Ground-Water and Surface-Water Elevations in the Fairbanks International Airport Area, 1990-94 


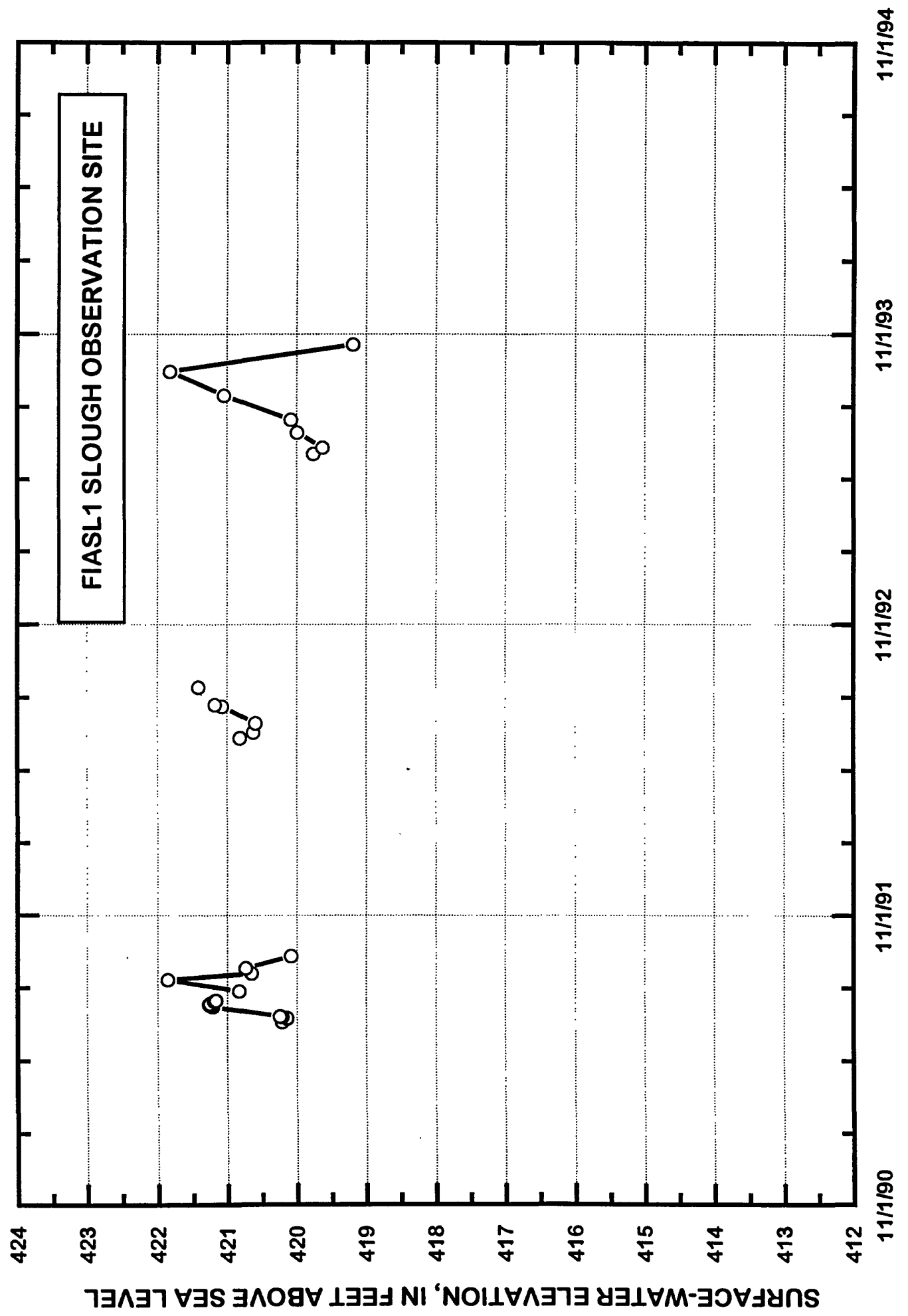




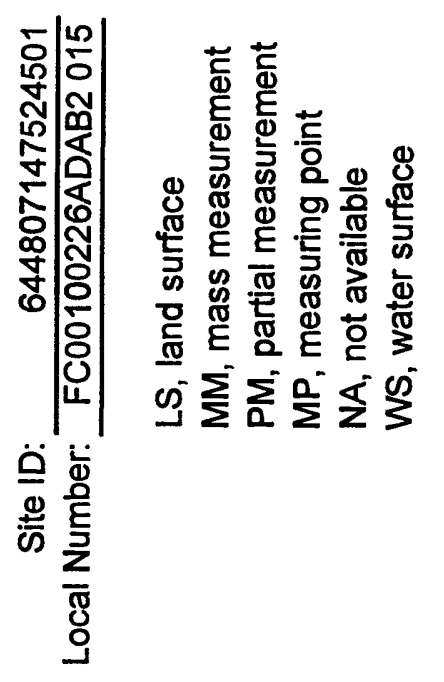

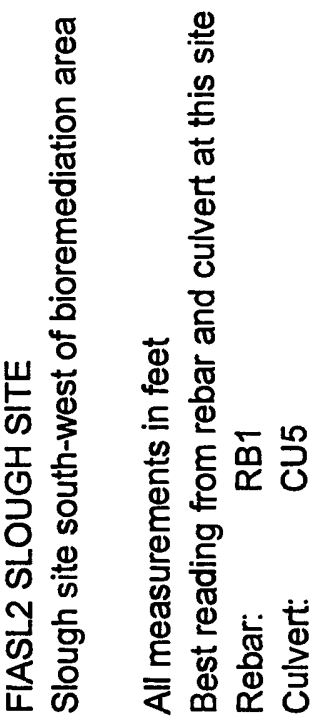

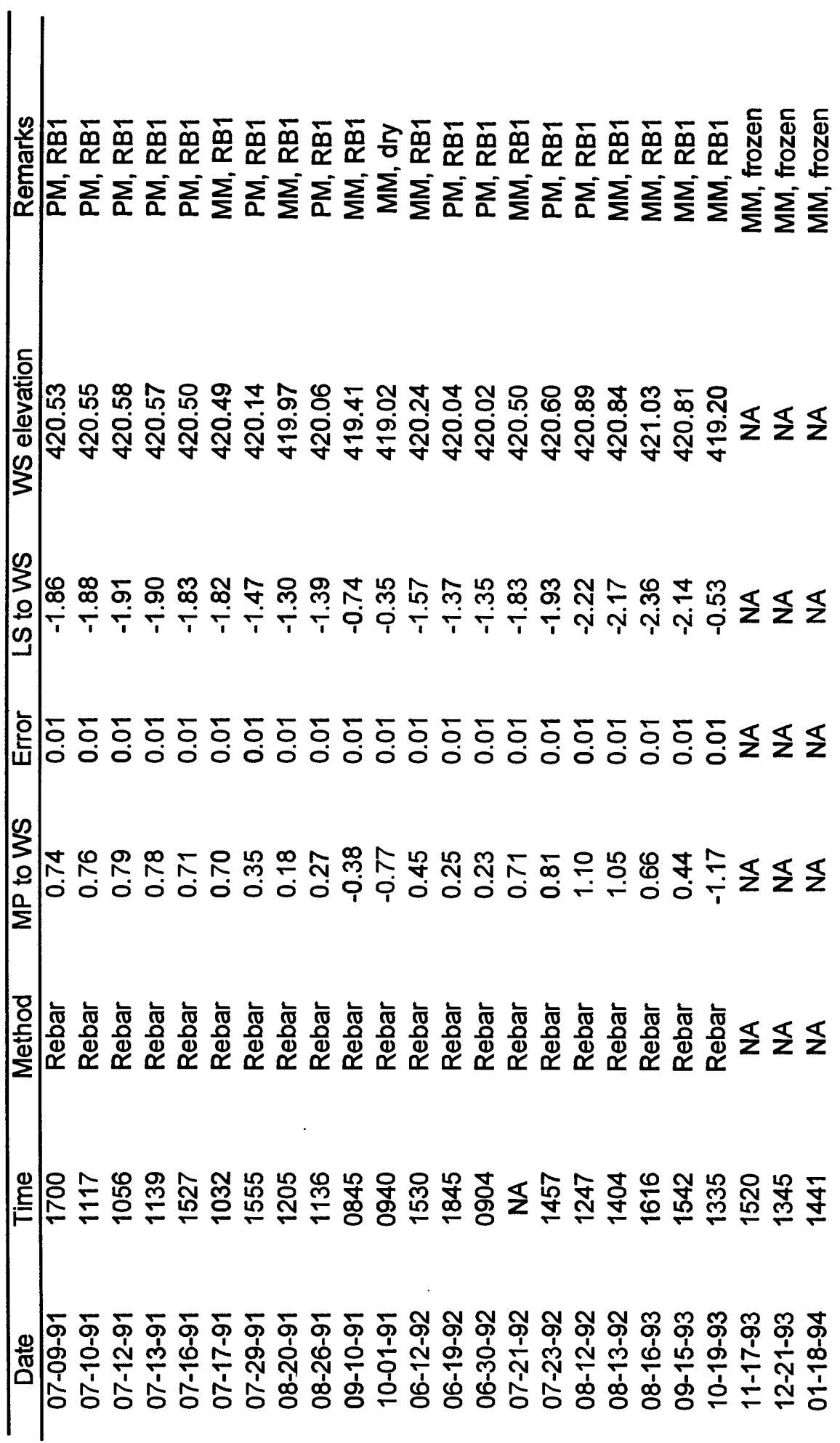




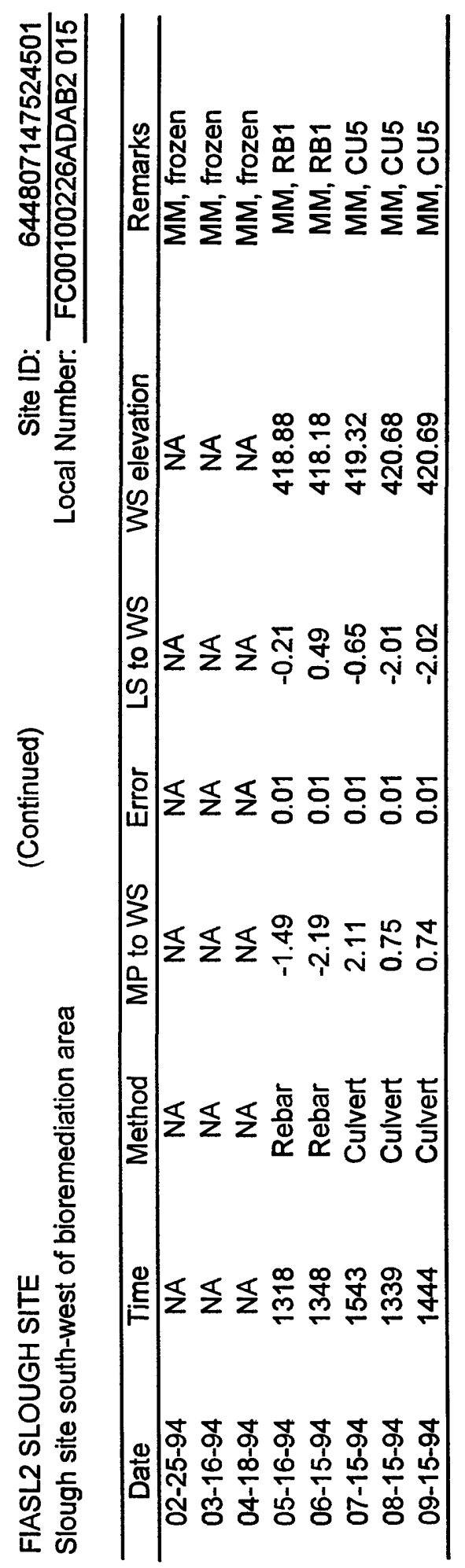


100 Ground-Water and Surface-Water Elevations in the Fairbanks International Airport Area, 1990-94 


$$
\mathbb{E}
$$



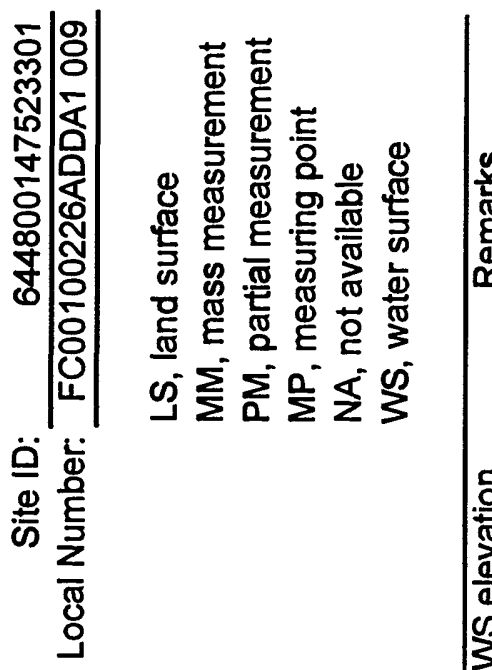

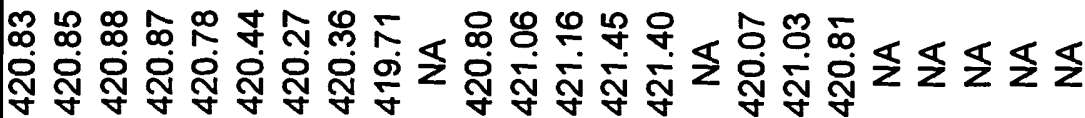

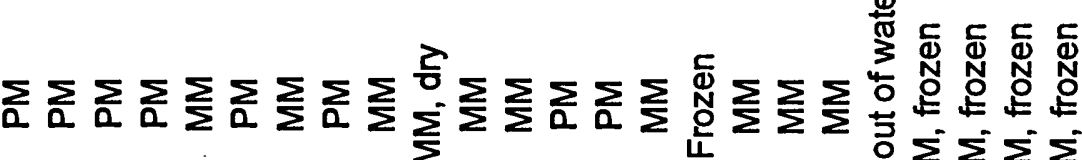
$\sum_{\sum}^{\infty} \sum_{\sum}^{\infty} \sum^{-}$

$\sum_{\substack{0 \\ 3}}$ क

究

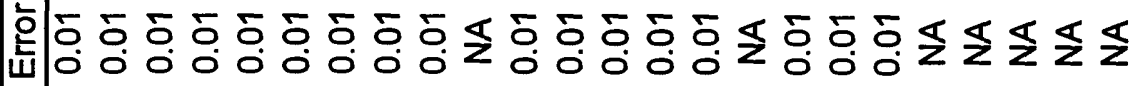

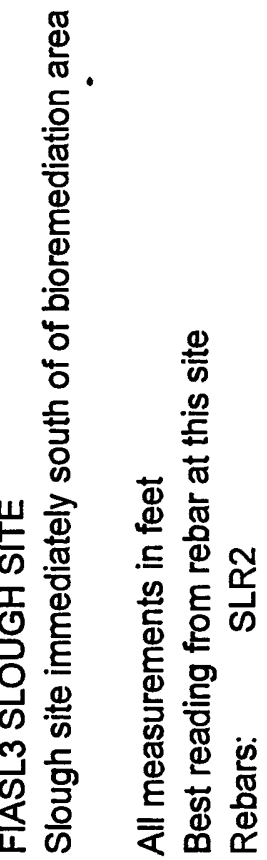

年

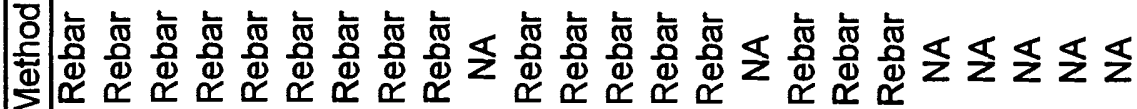

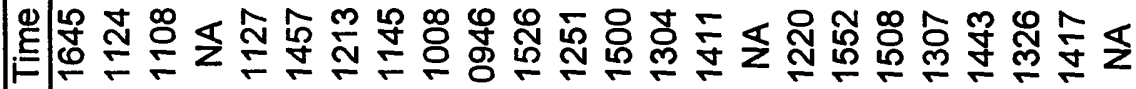

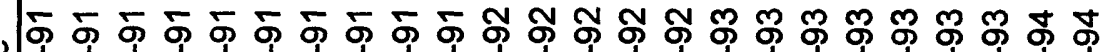

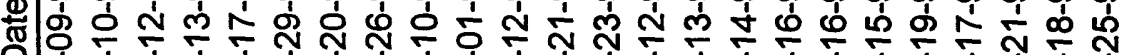
|동 


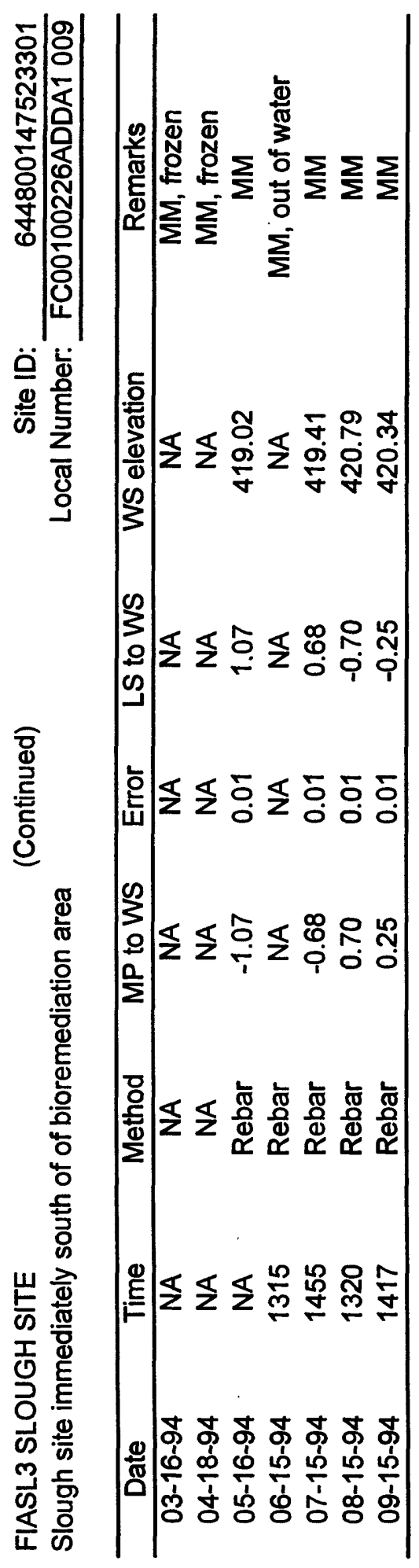




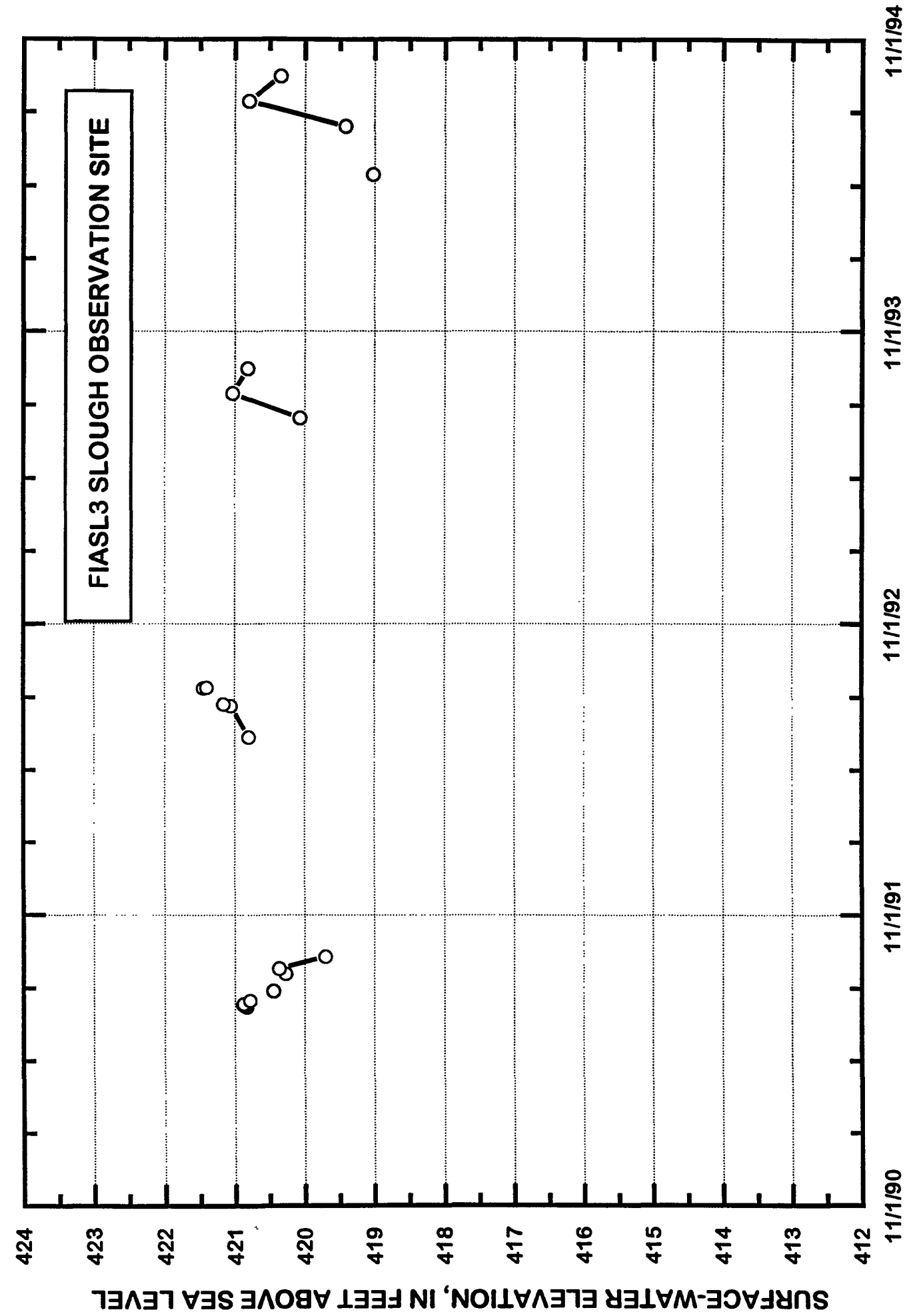




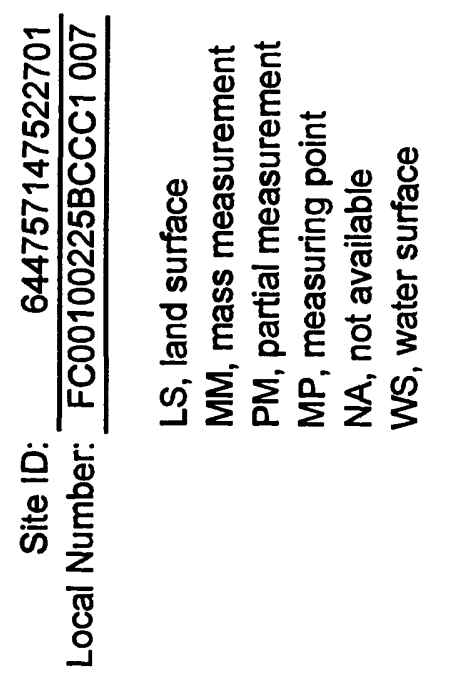

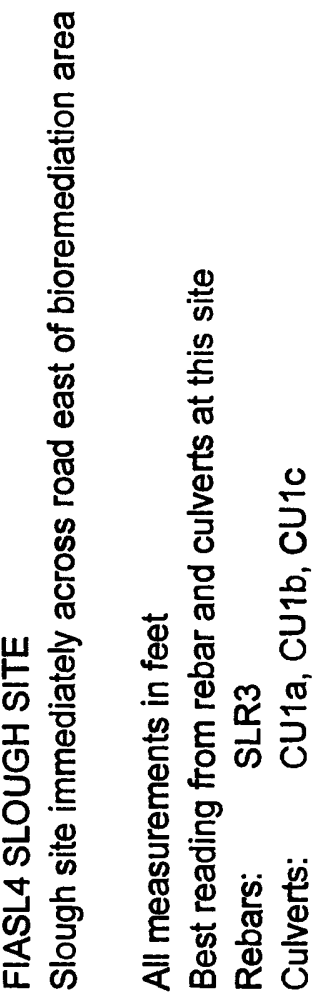

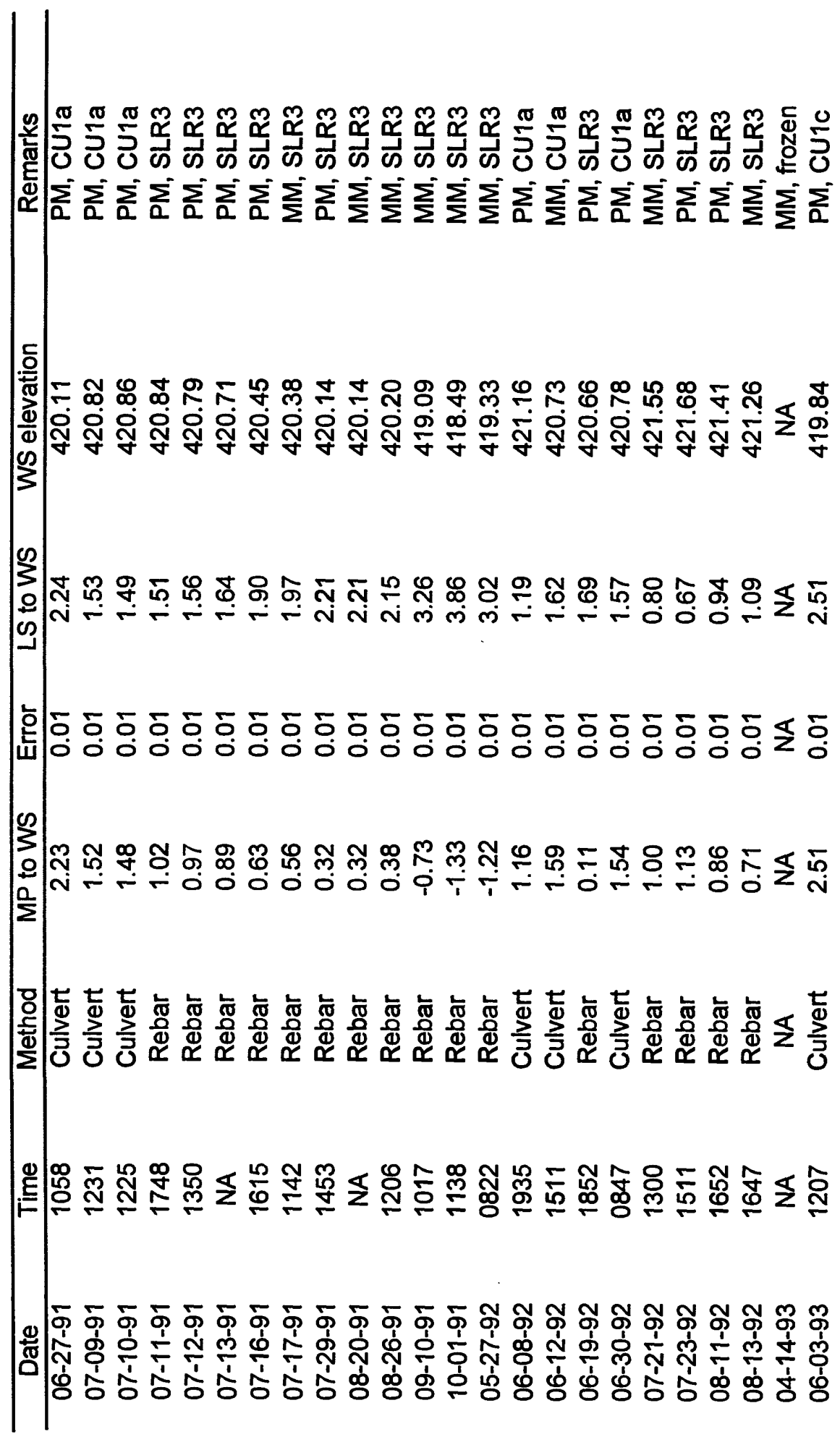




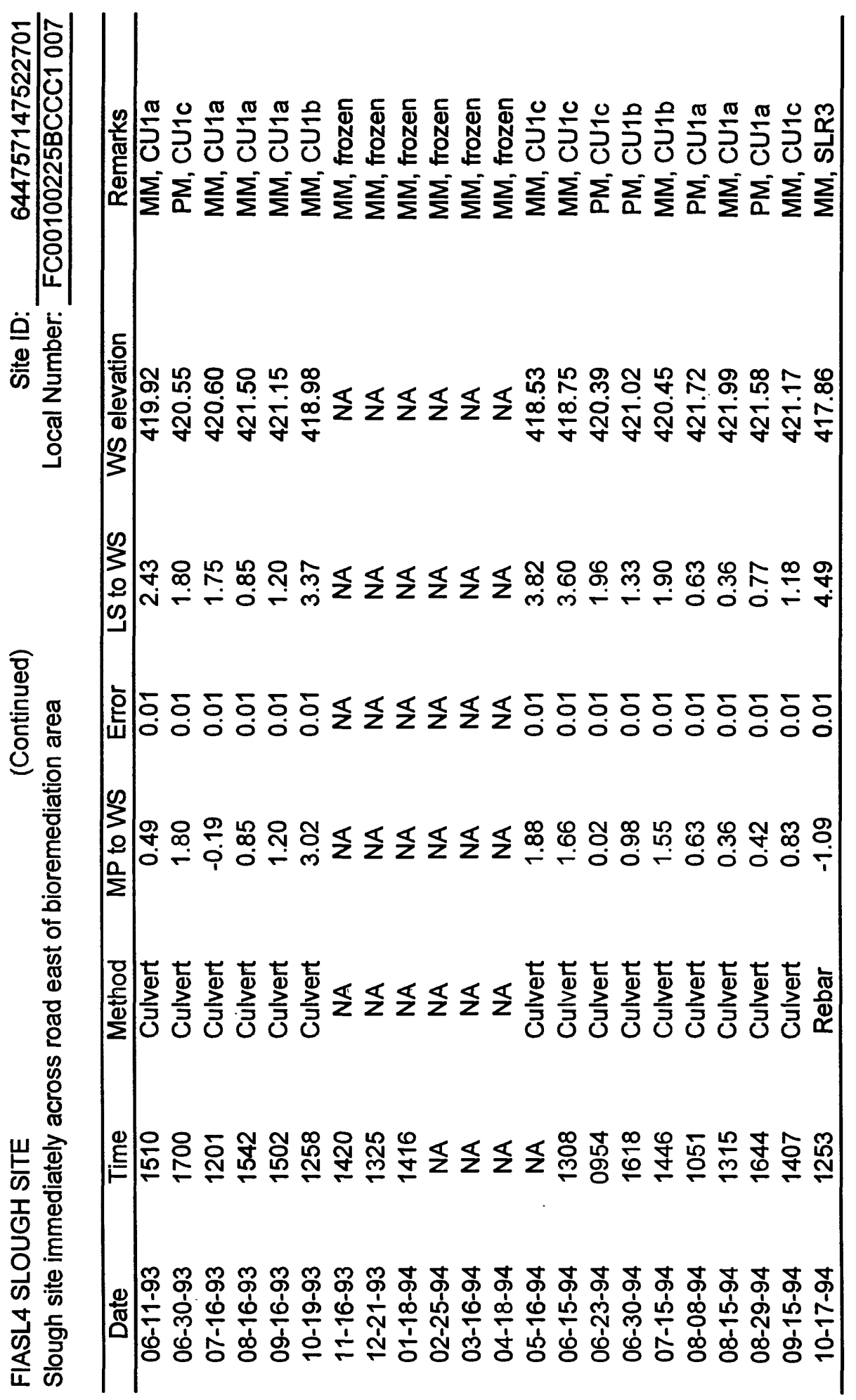




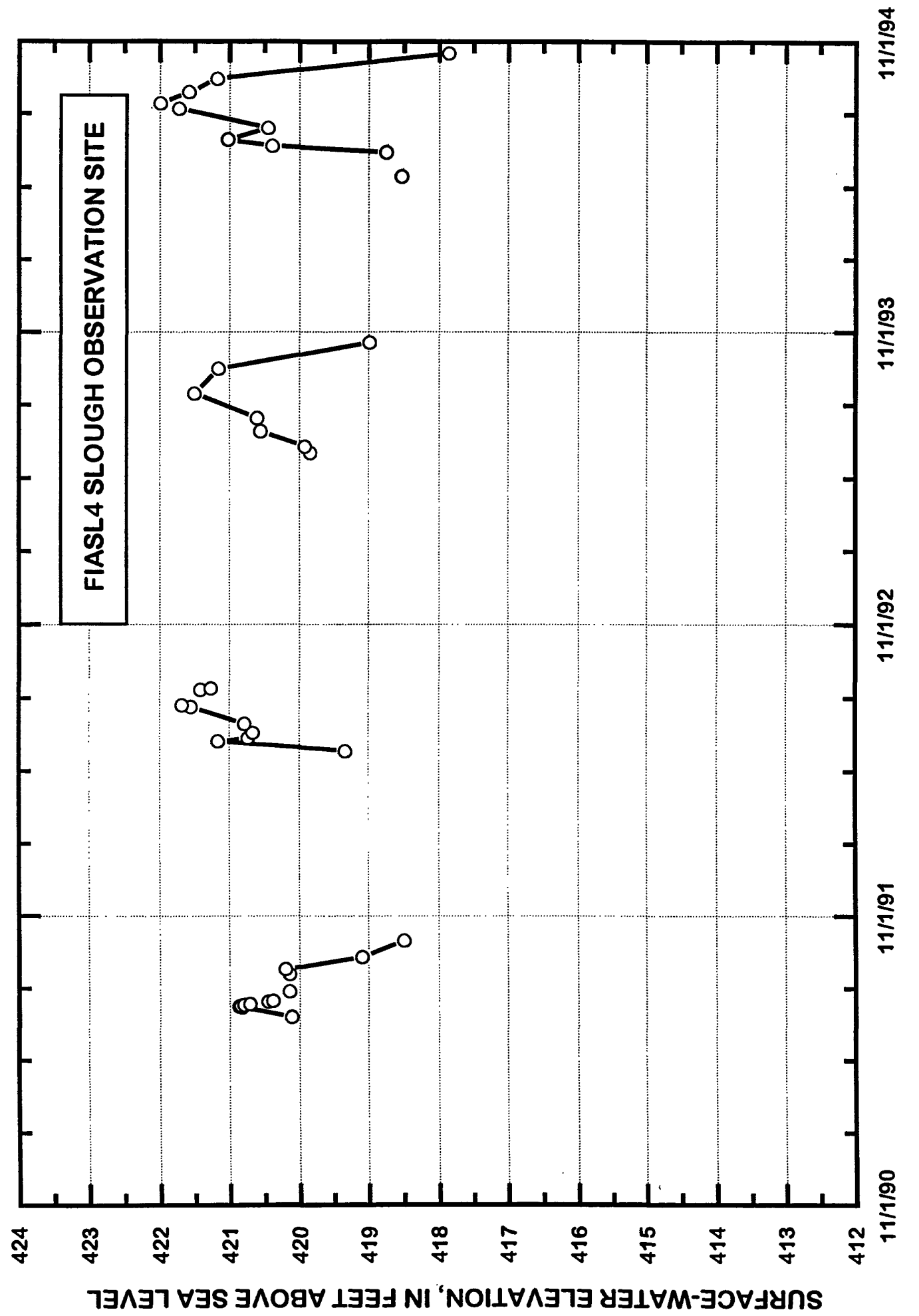




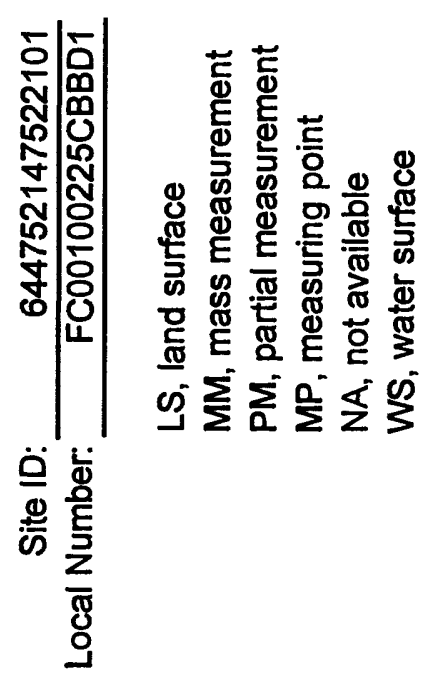

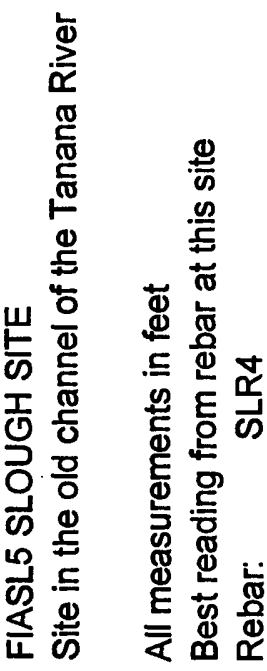

$\hat{\infty}$
$\infty$
$\stackrel{+}{+}$

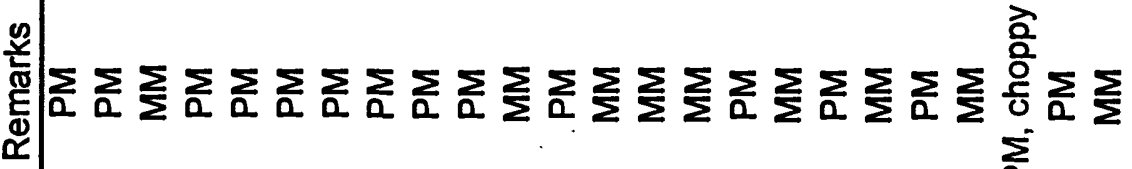

历̄

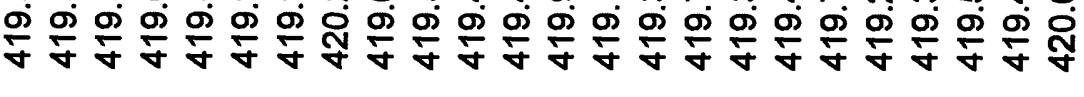

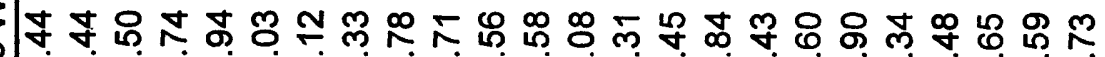
c)

逍

年 莳 立

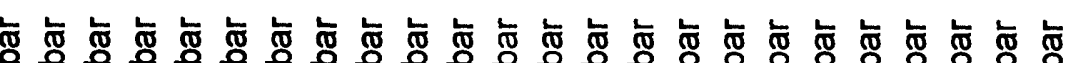

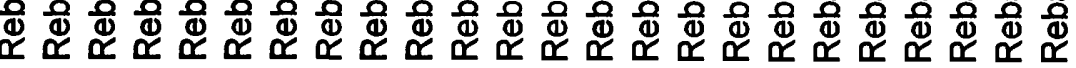

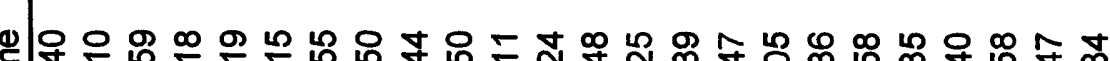

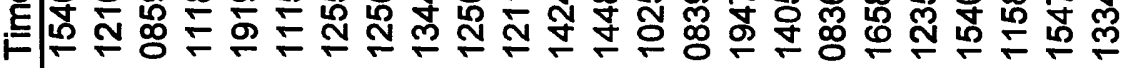

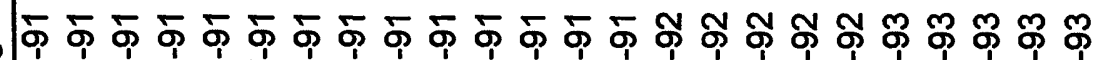

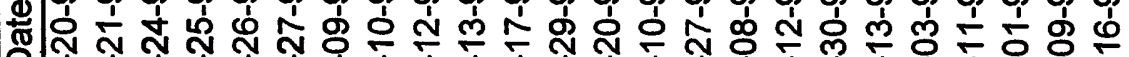

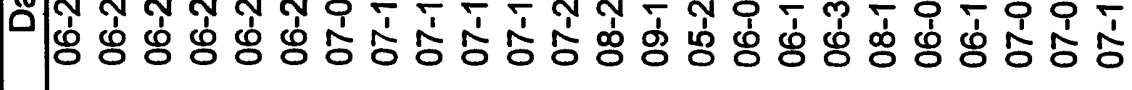




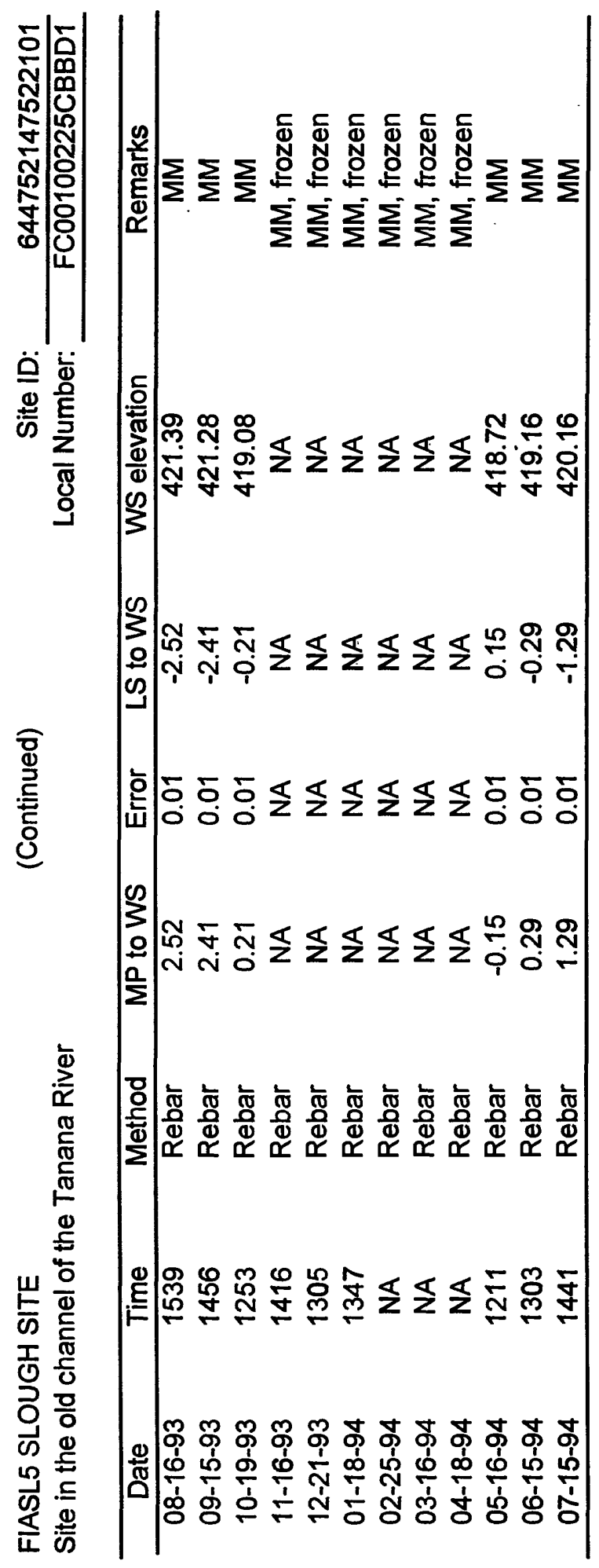




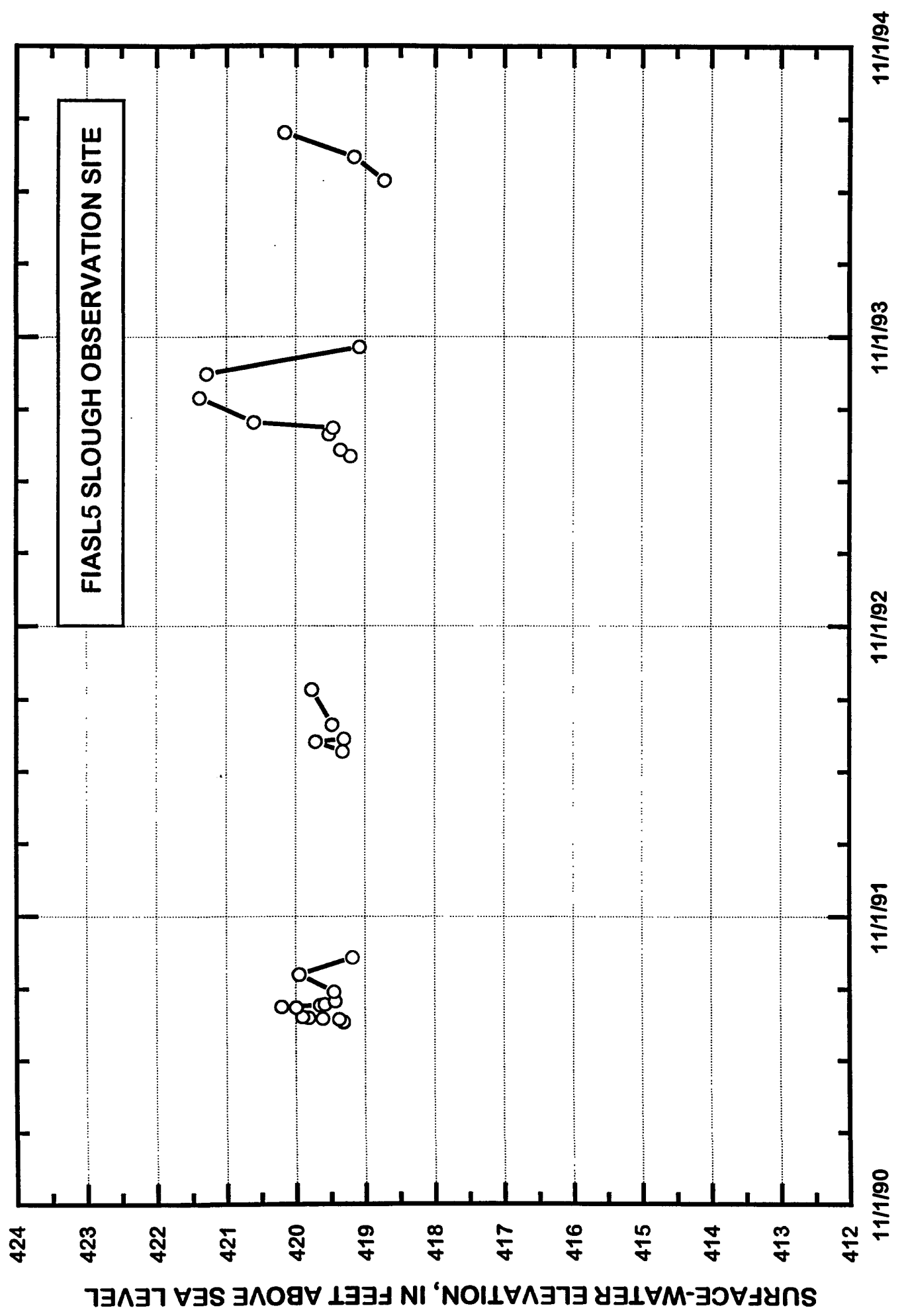




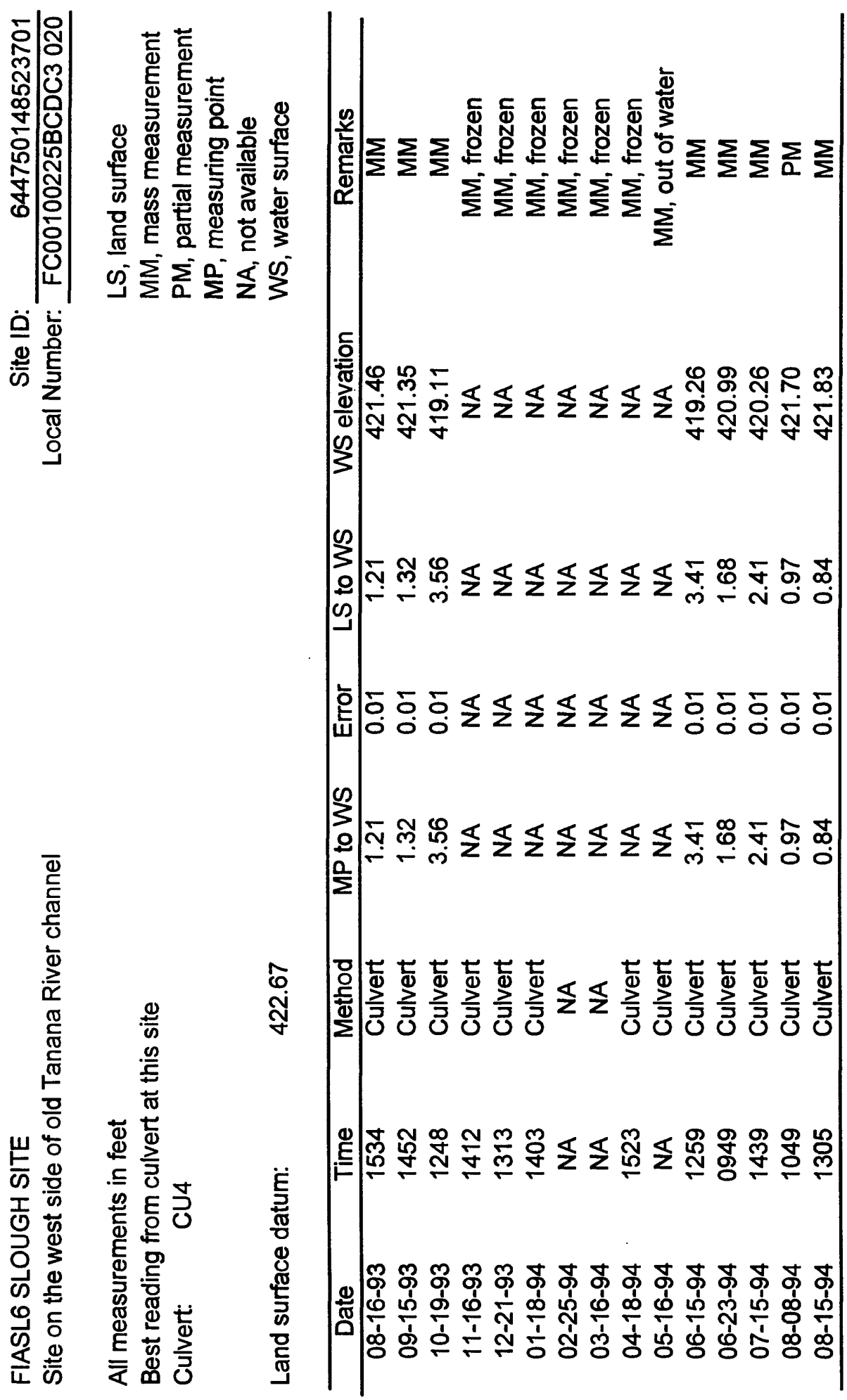




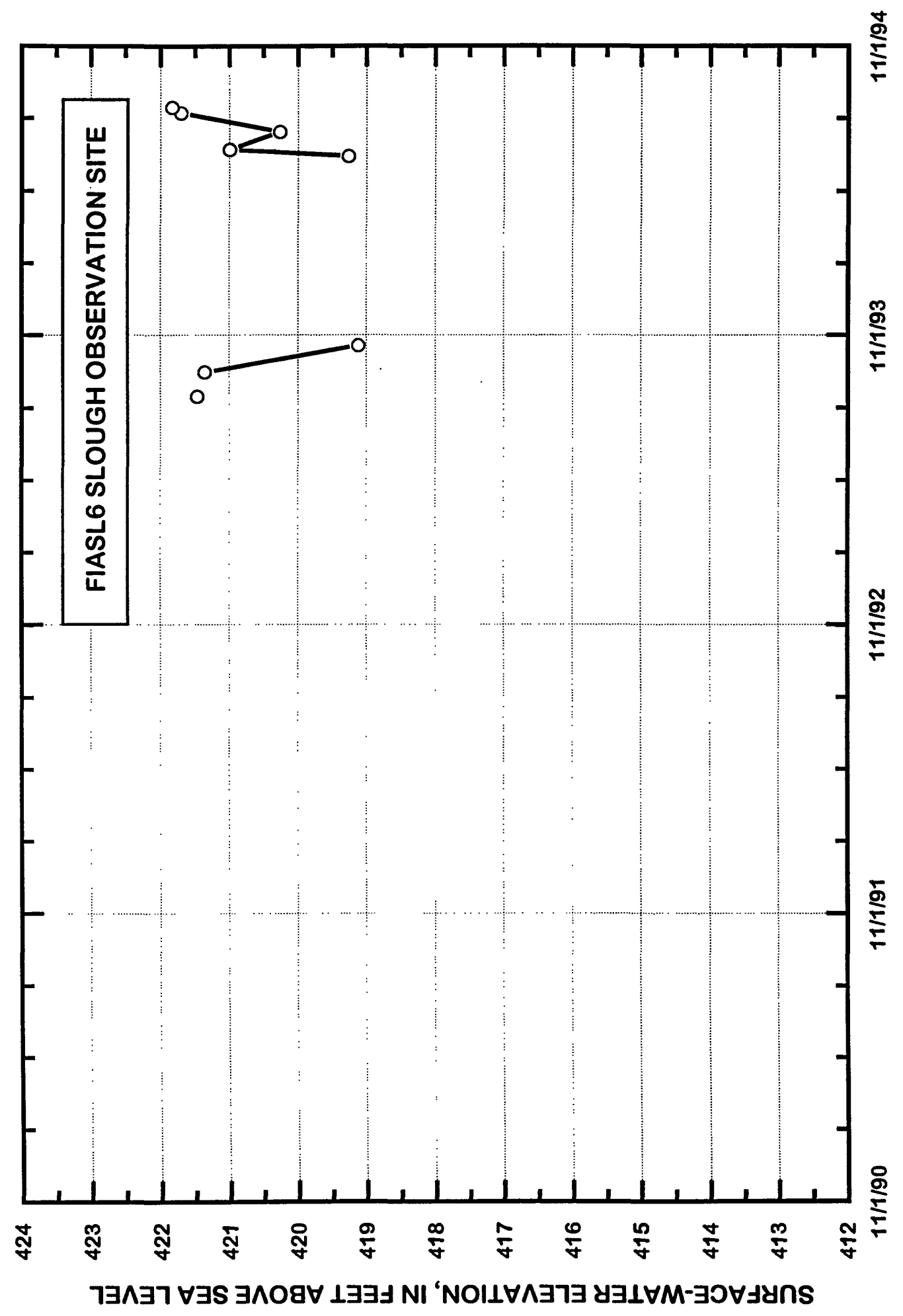




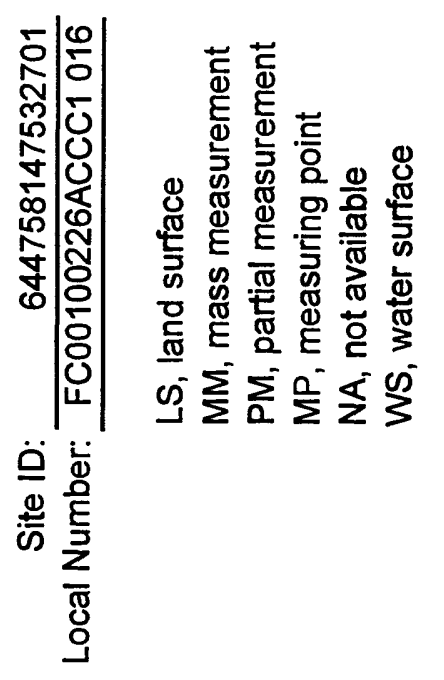

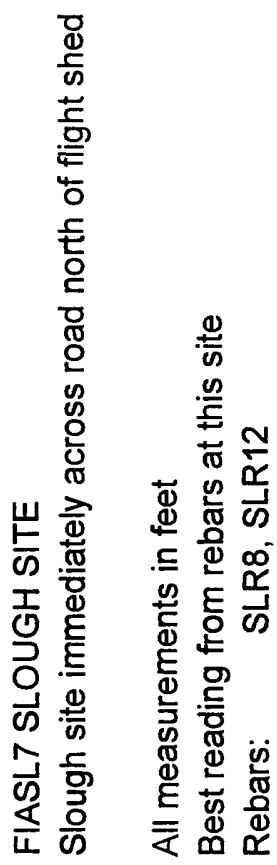

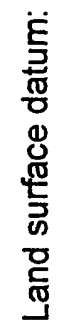

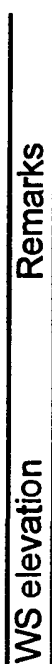

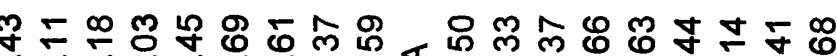

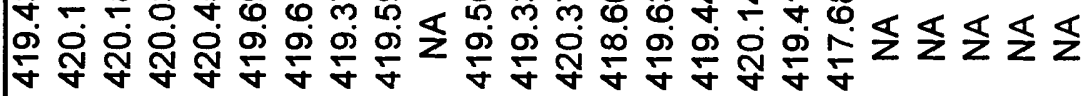
舟

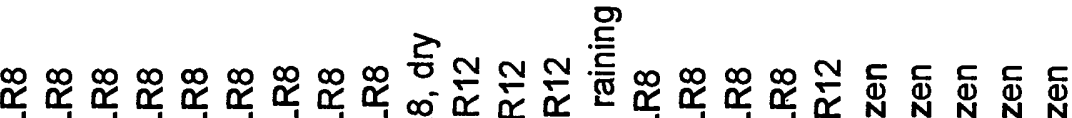
の あ

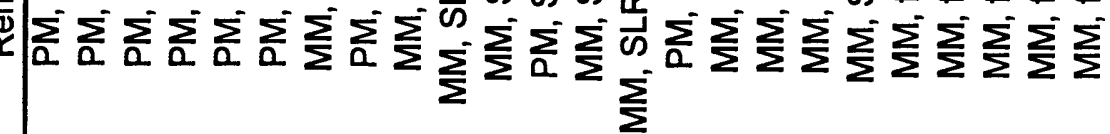
(1)

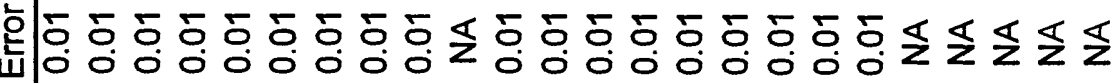

$\stackrel{3}{3}$

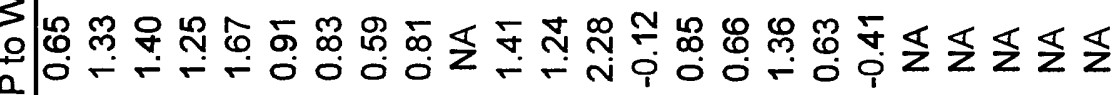
$\Sigma$

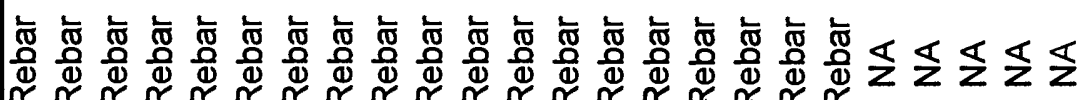

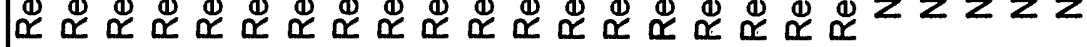

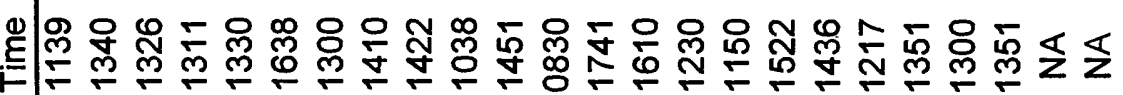

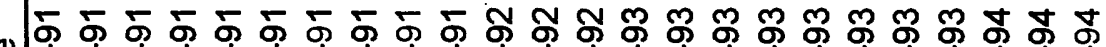

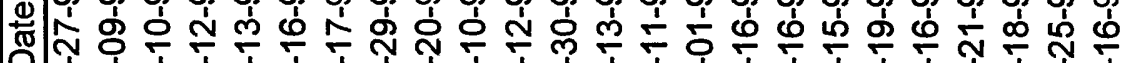

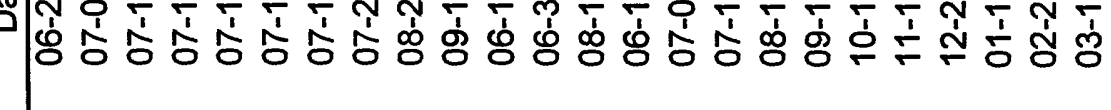




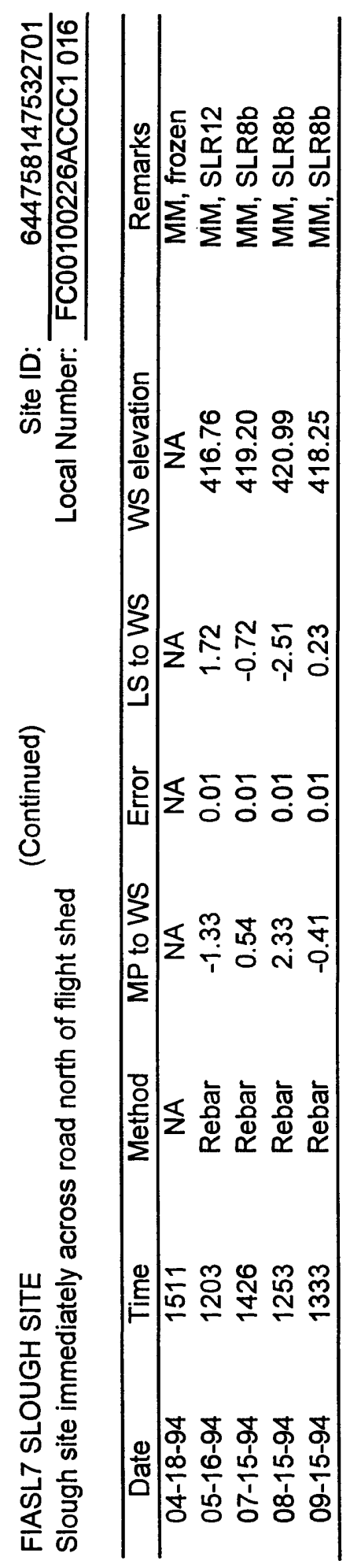


118 Ground-Water and Surface-Water Elevations in the Fairbanks International Airport Area, 1990-94 


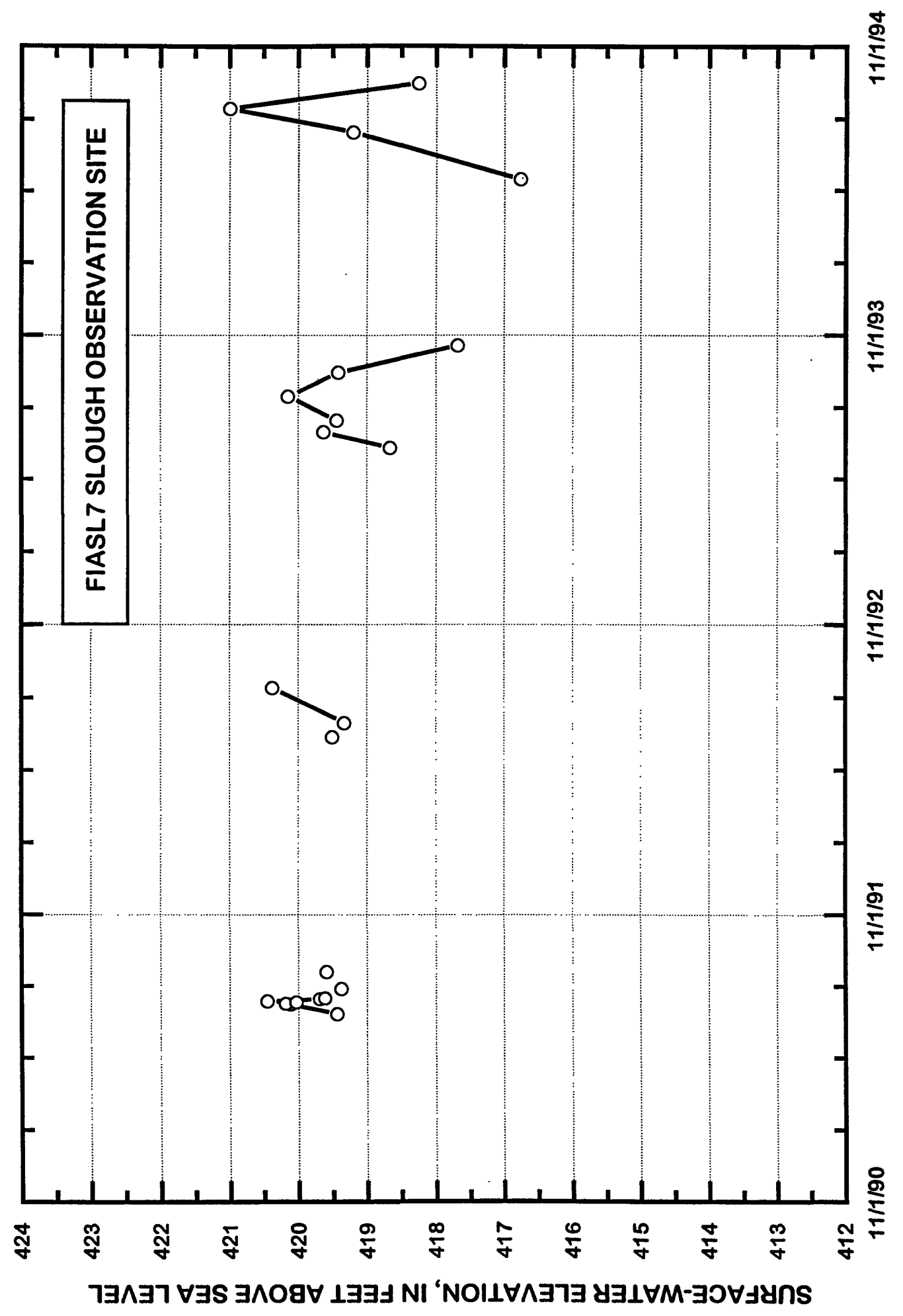




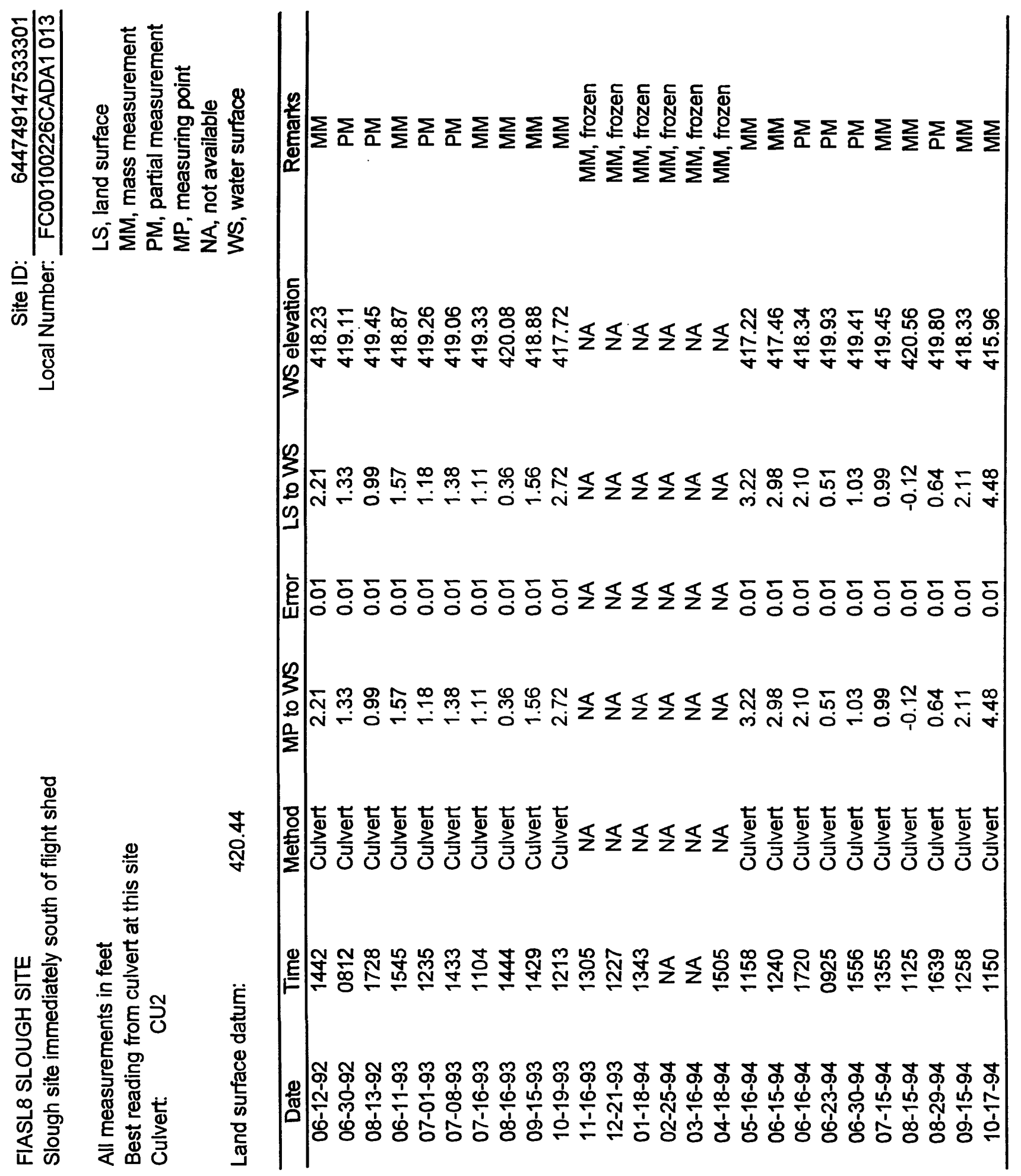




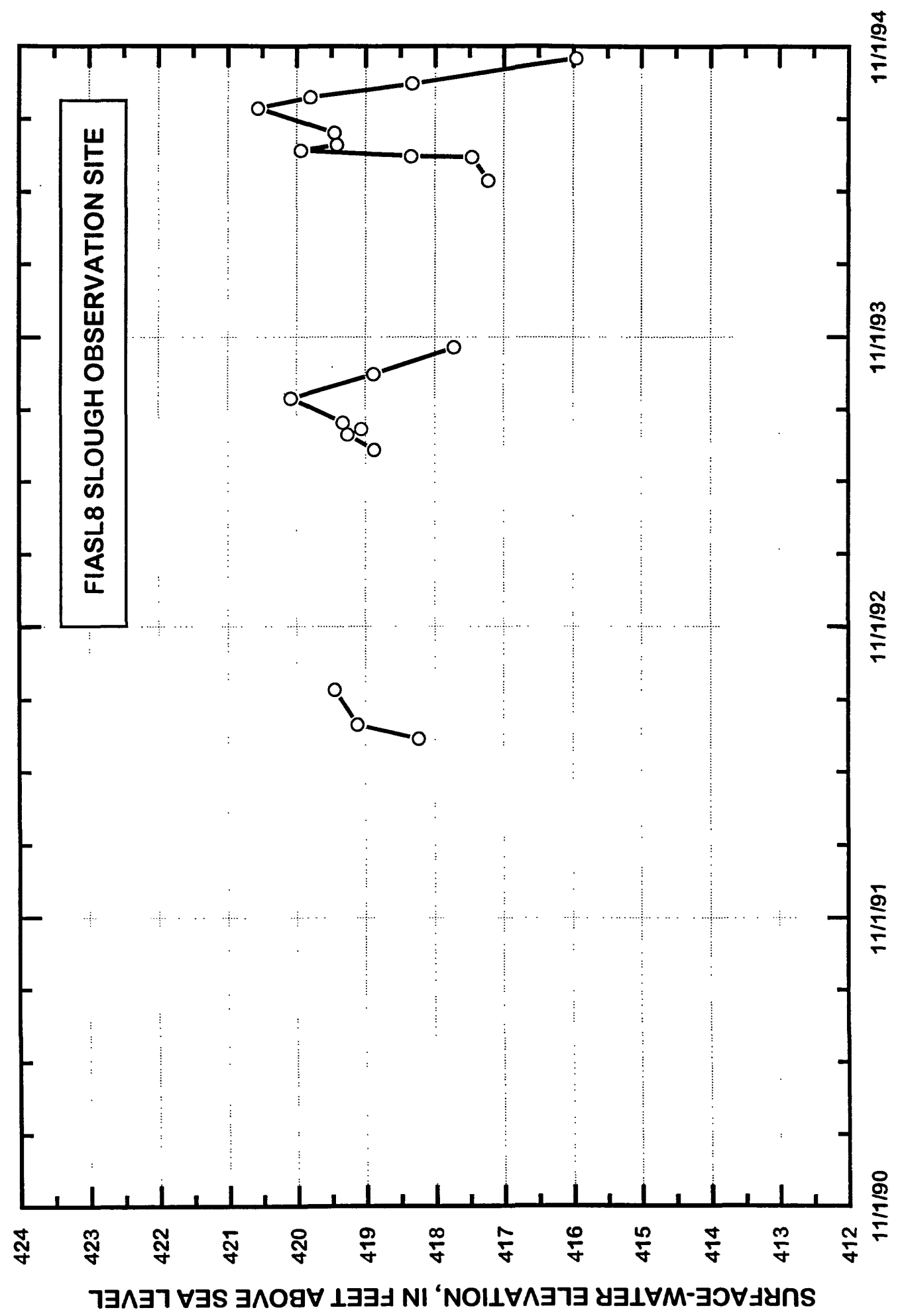




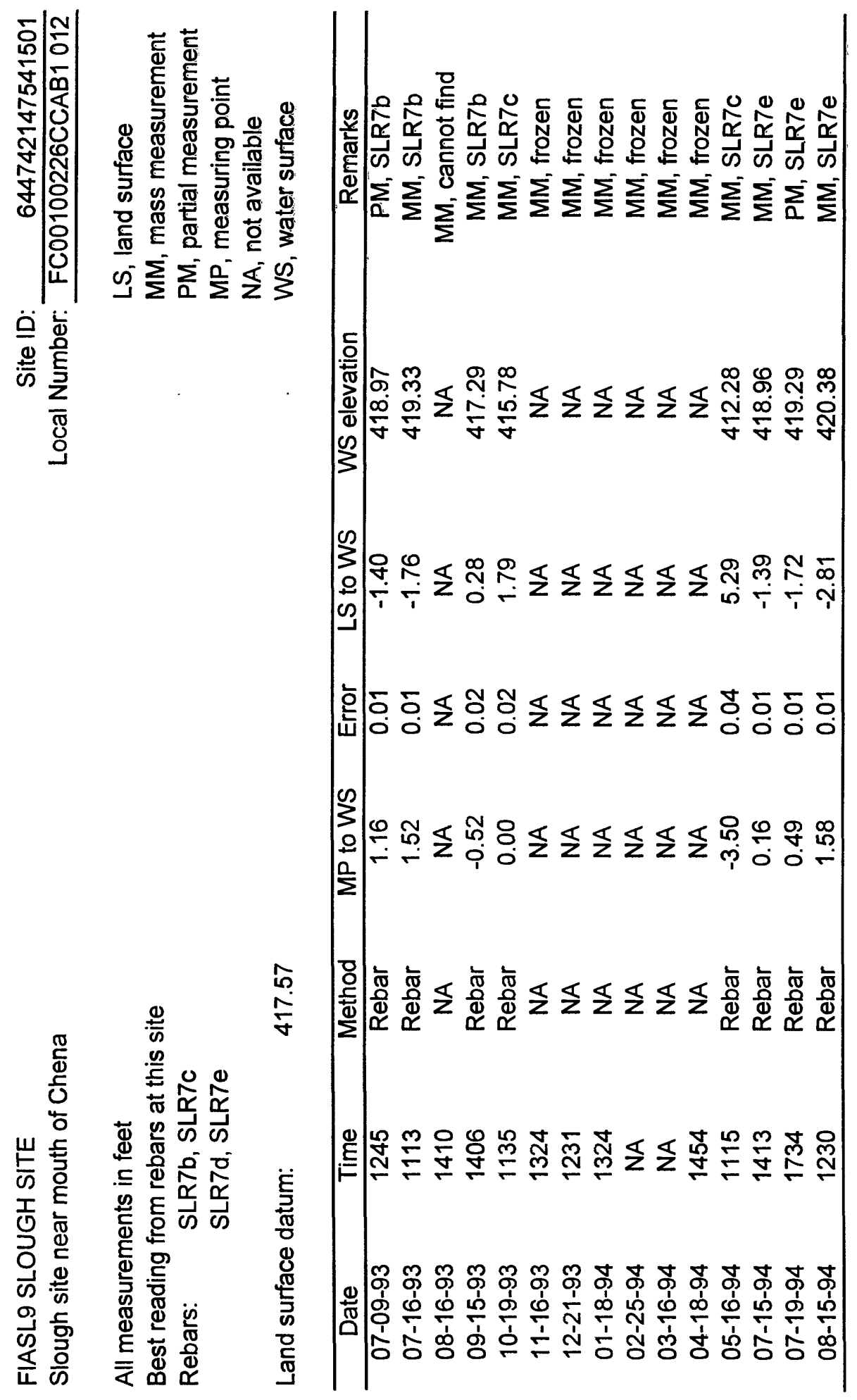




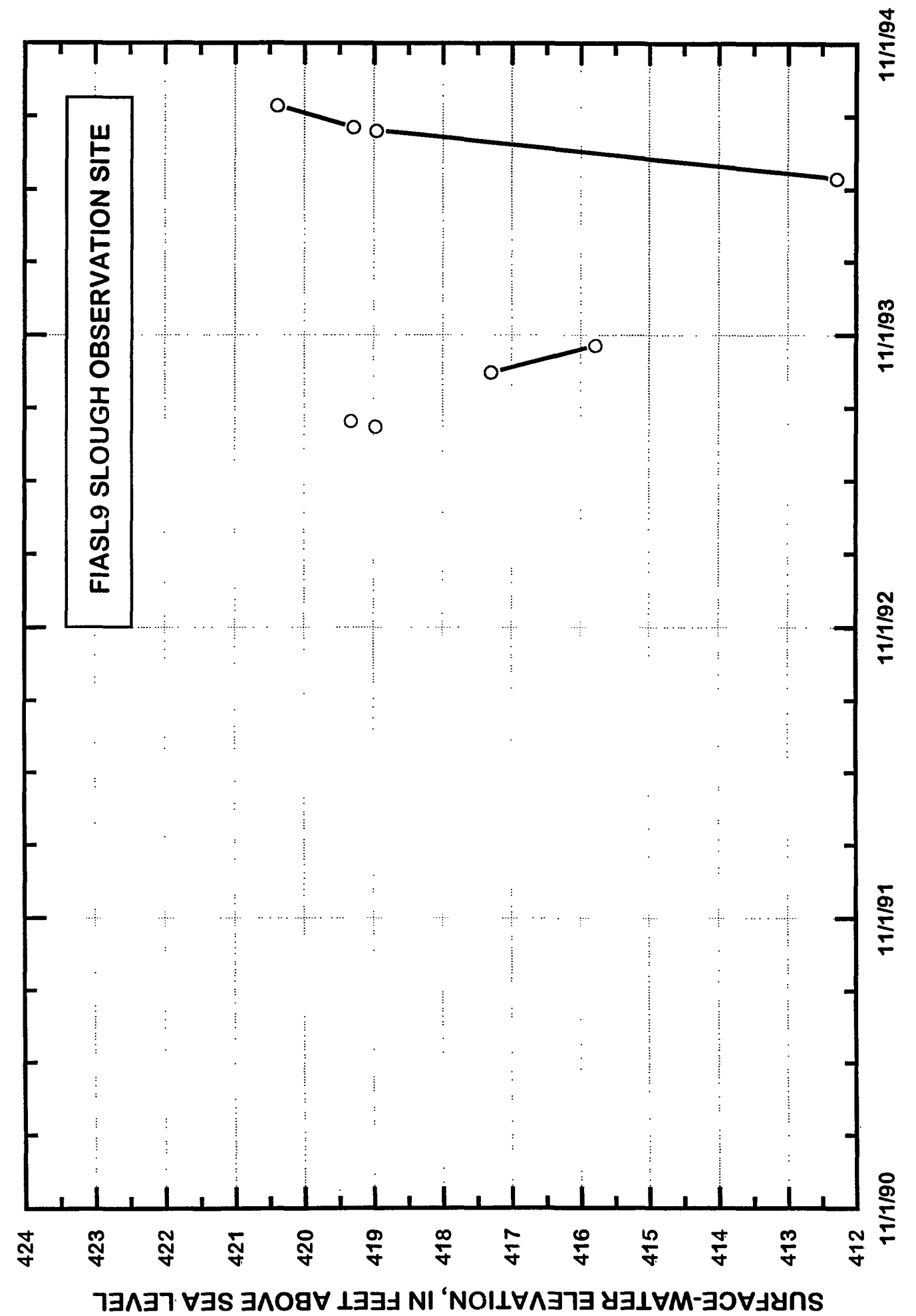




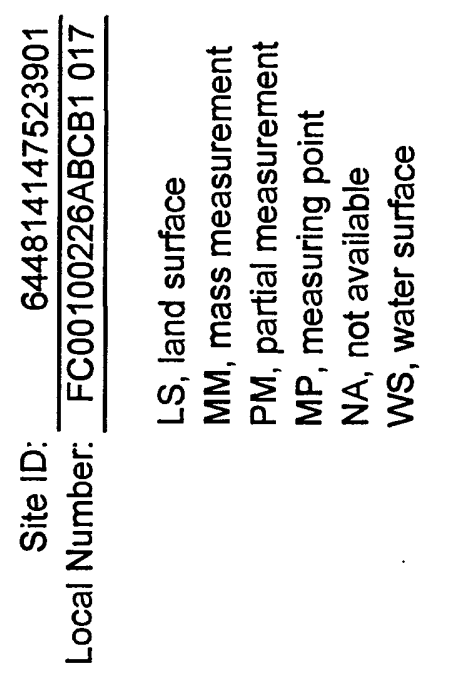

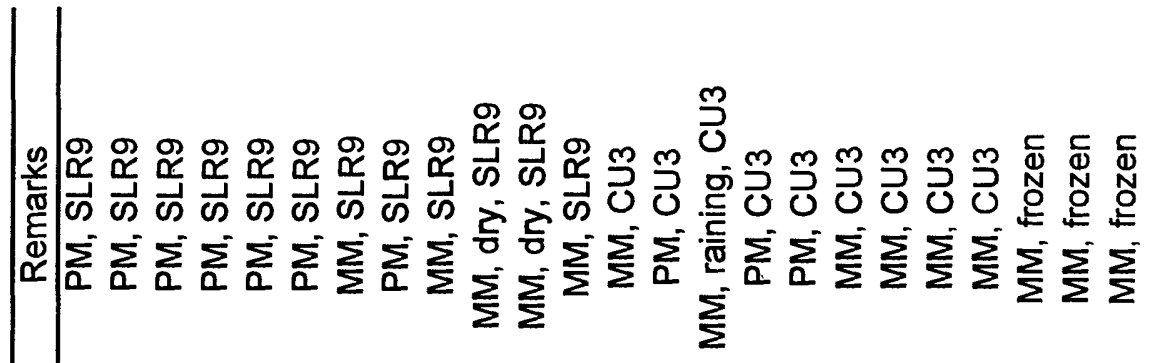

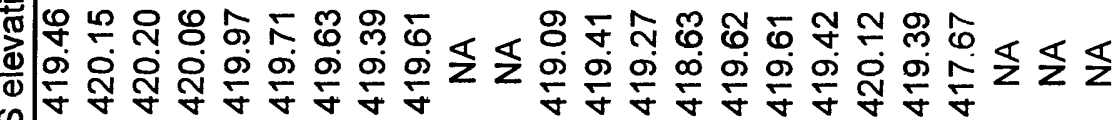

$\stackrel{\infty}{3}$

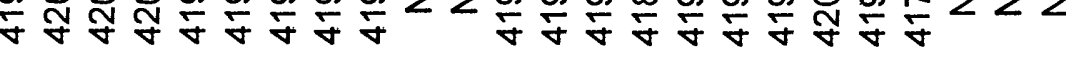

$\stackrel{3}{3}$

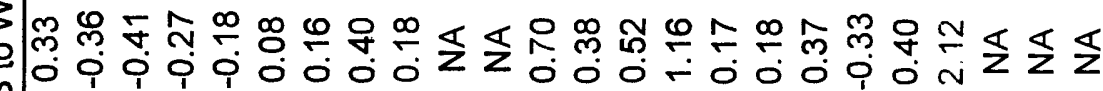

ज

产豞

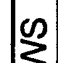

|

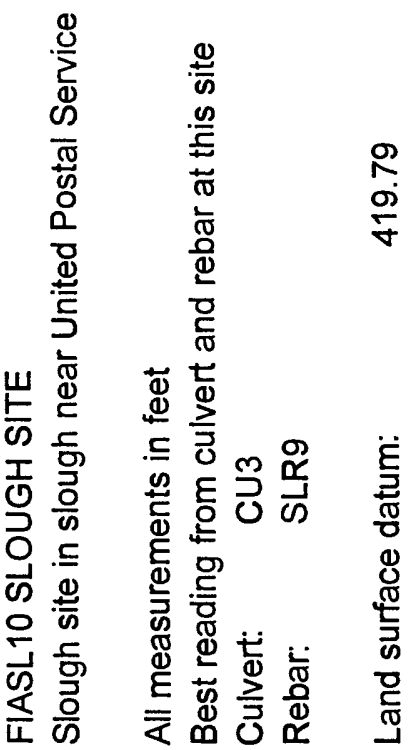

$\Sigma$

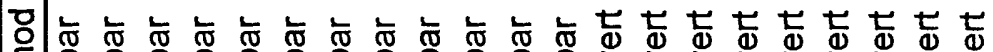

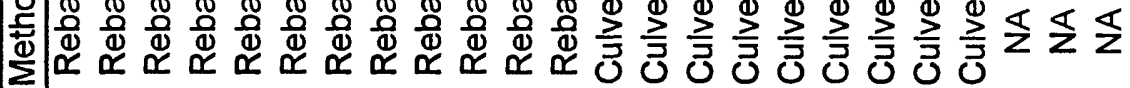

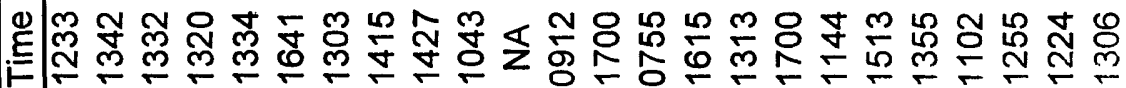

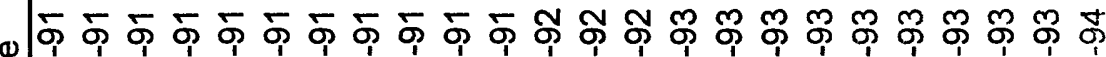

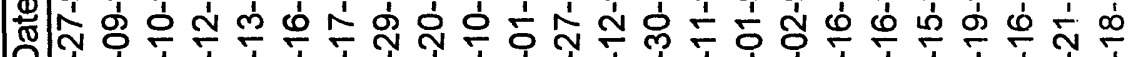

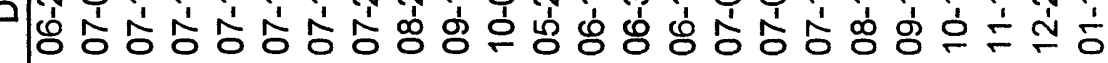




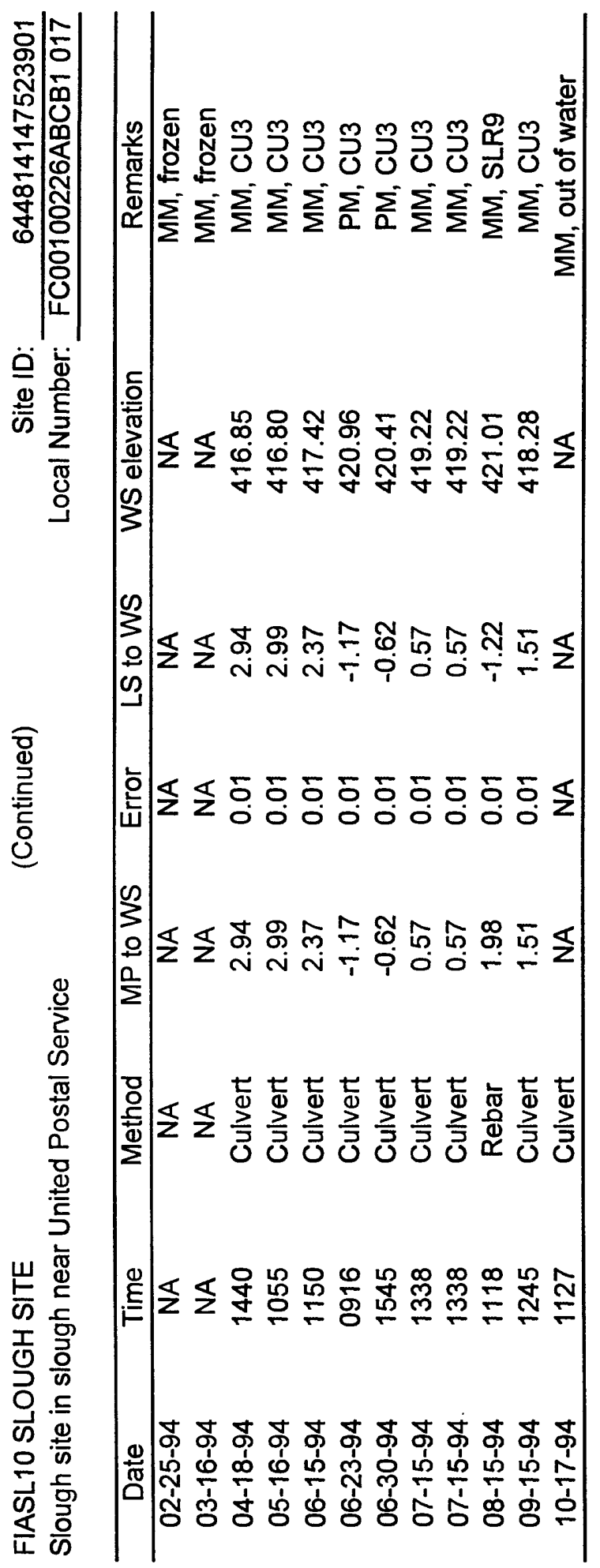




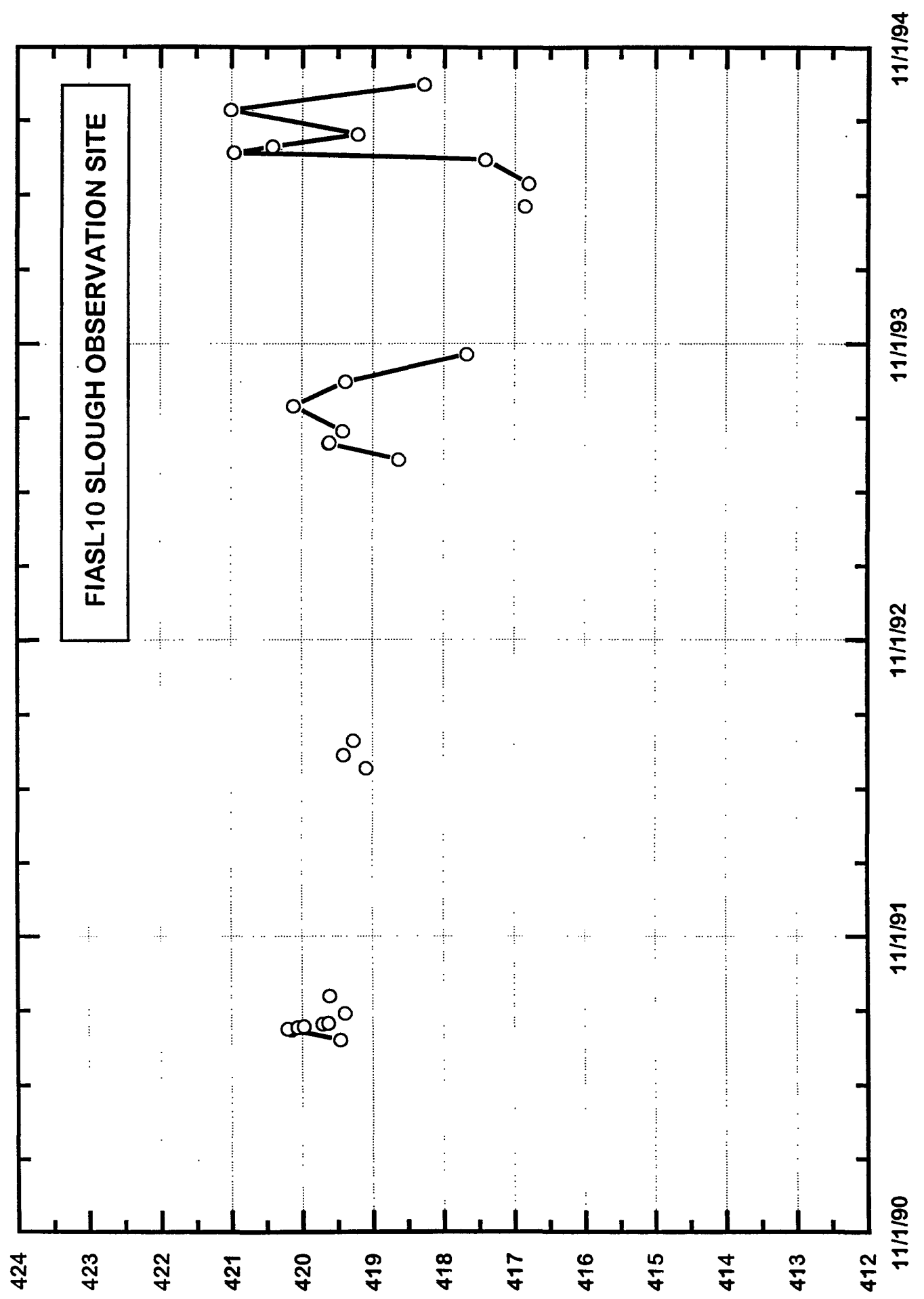

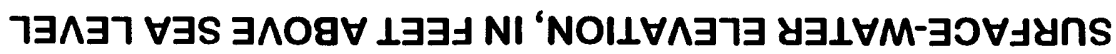




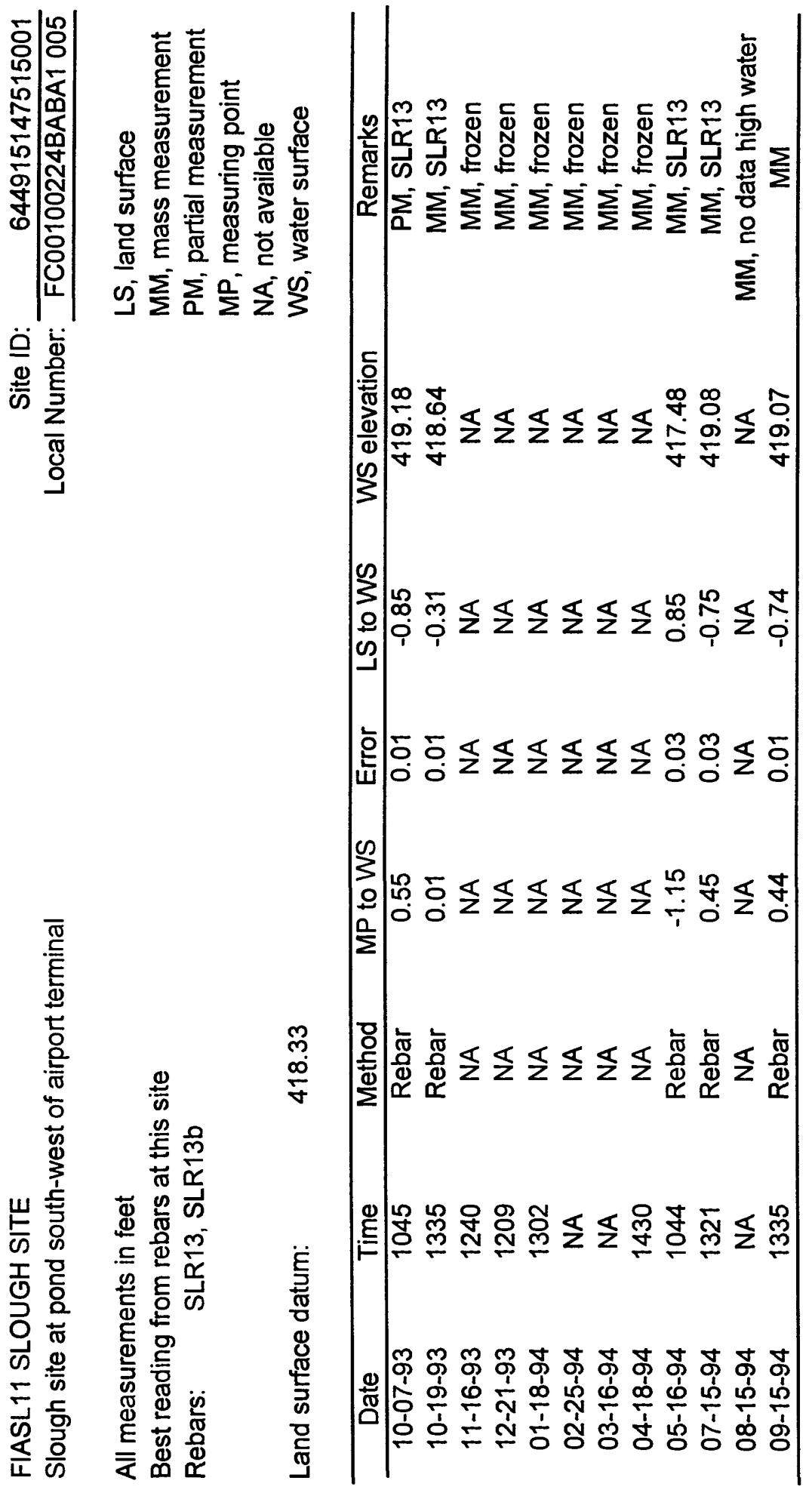




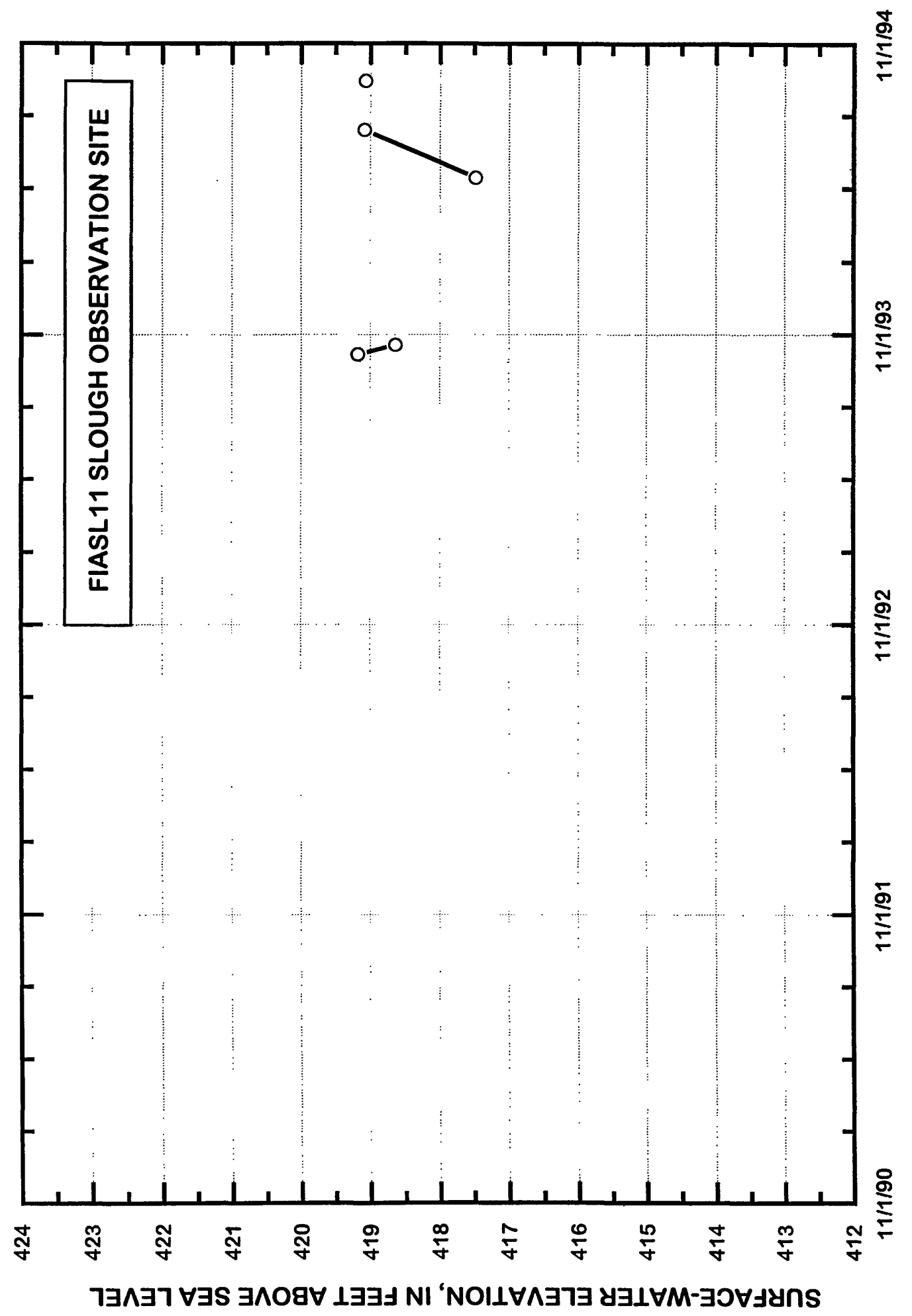




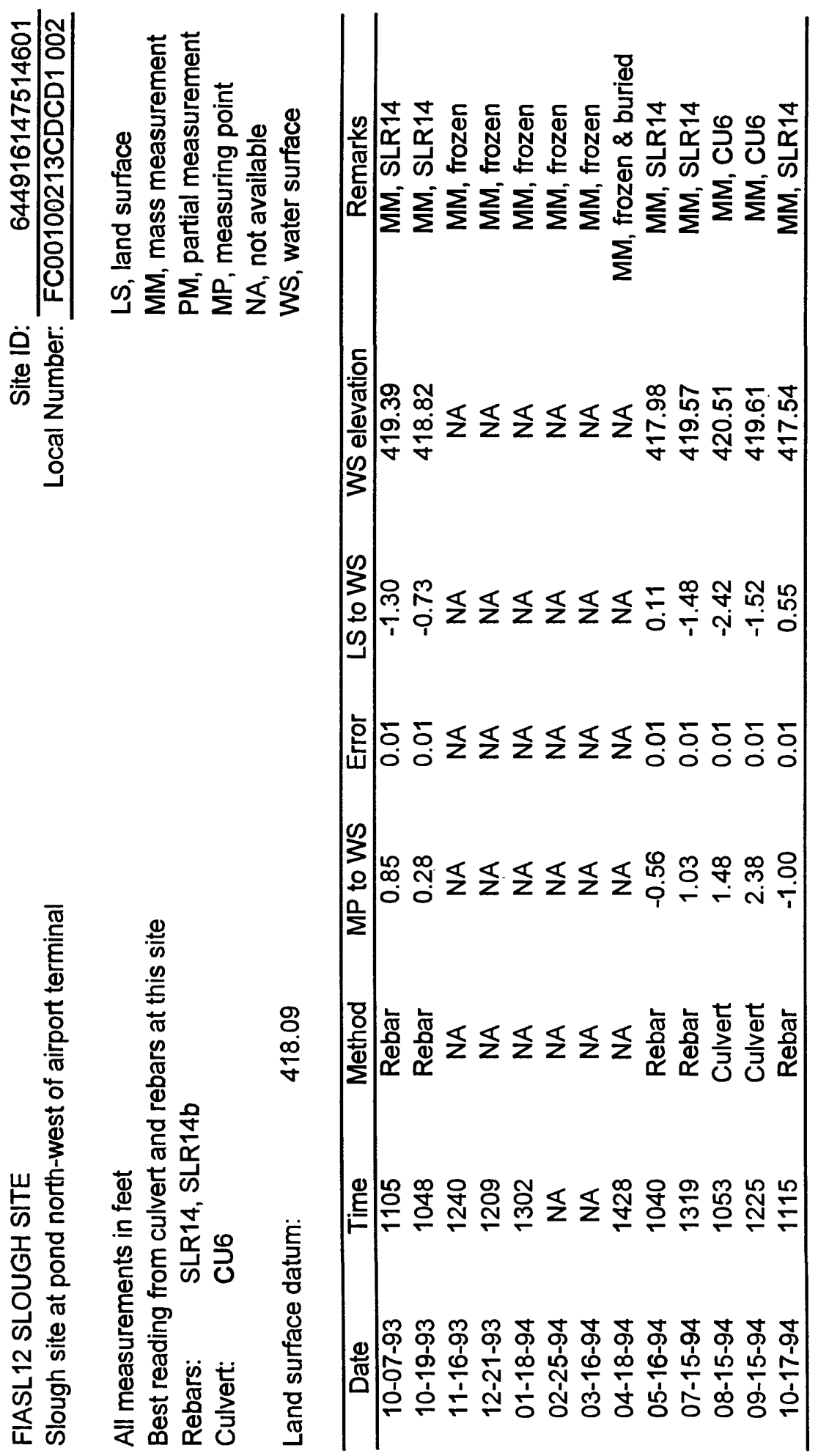




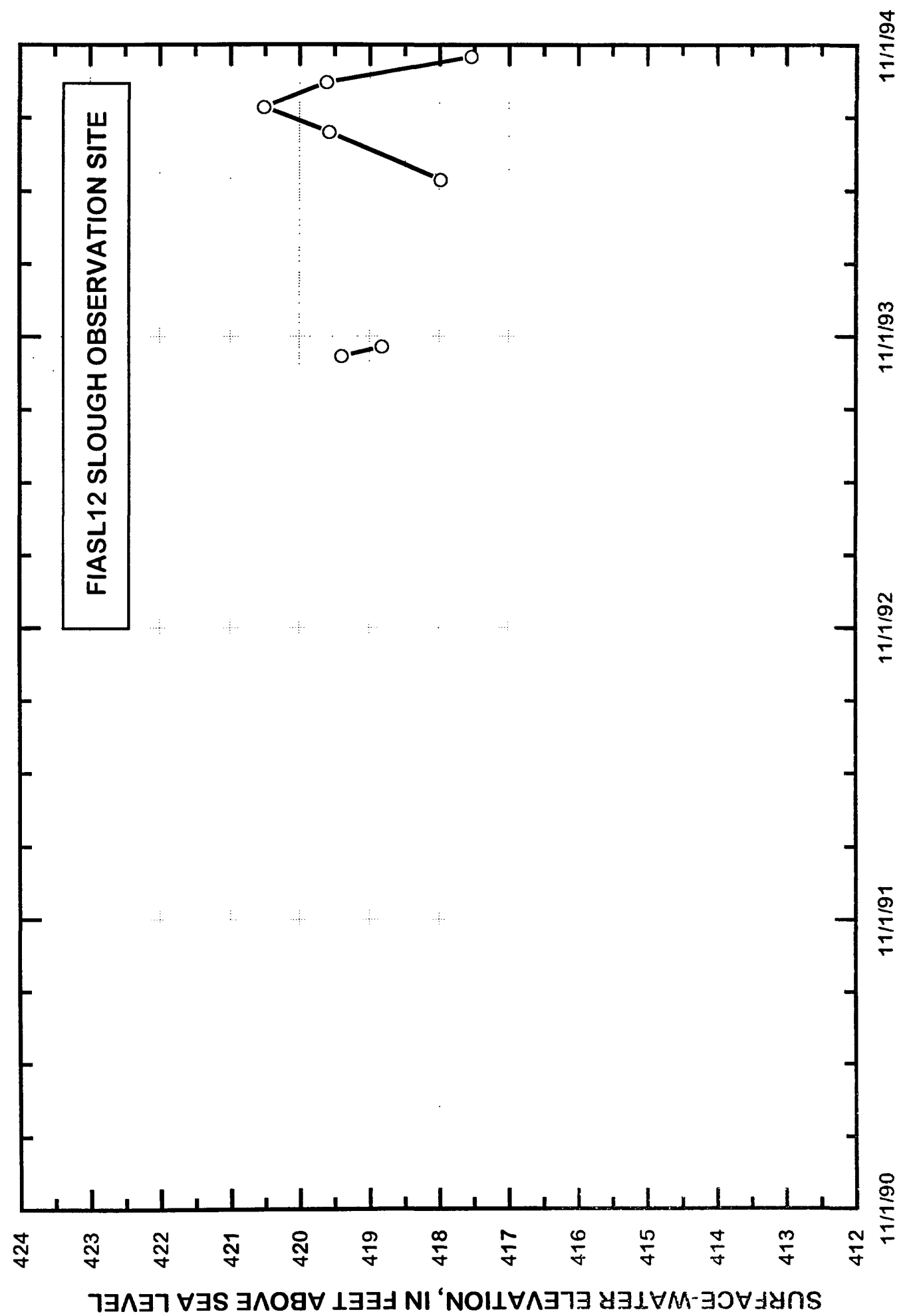




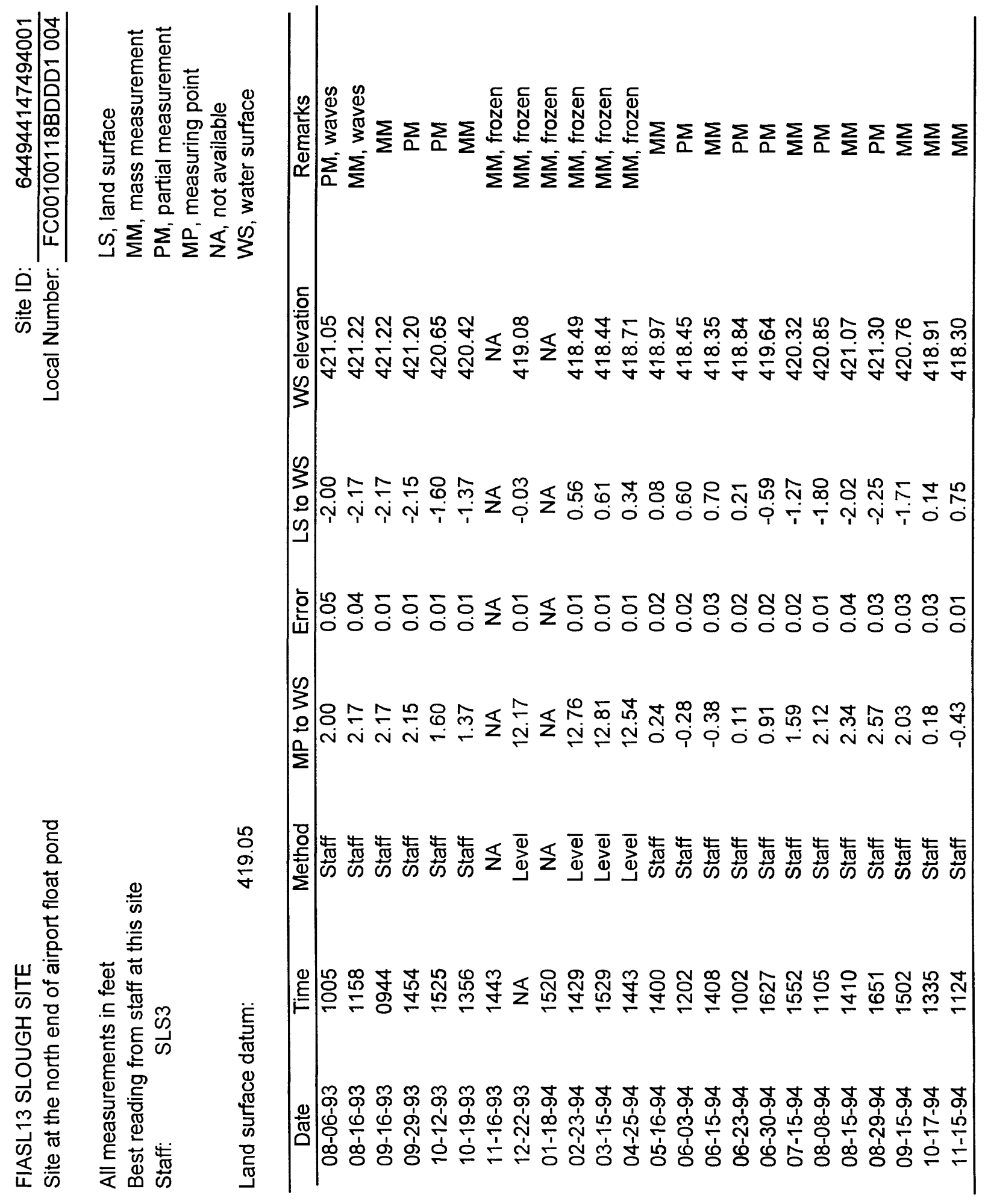




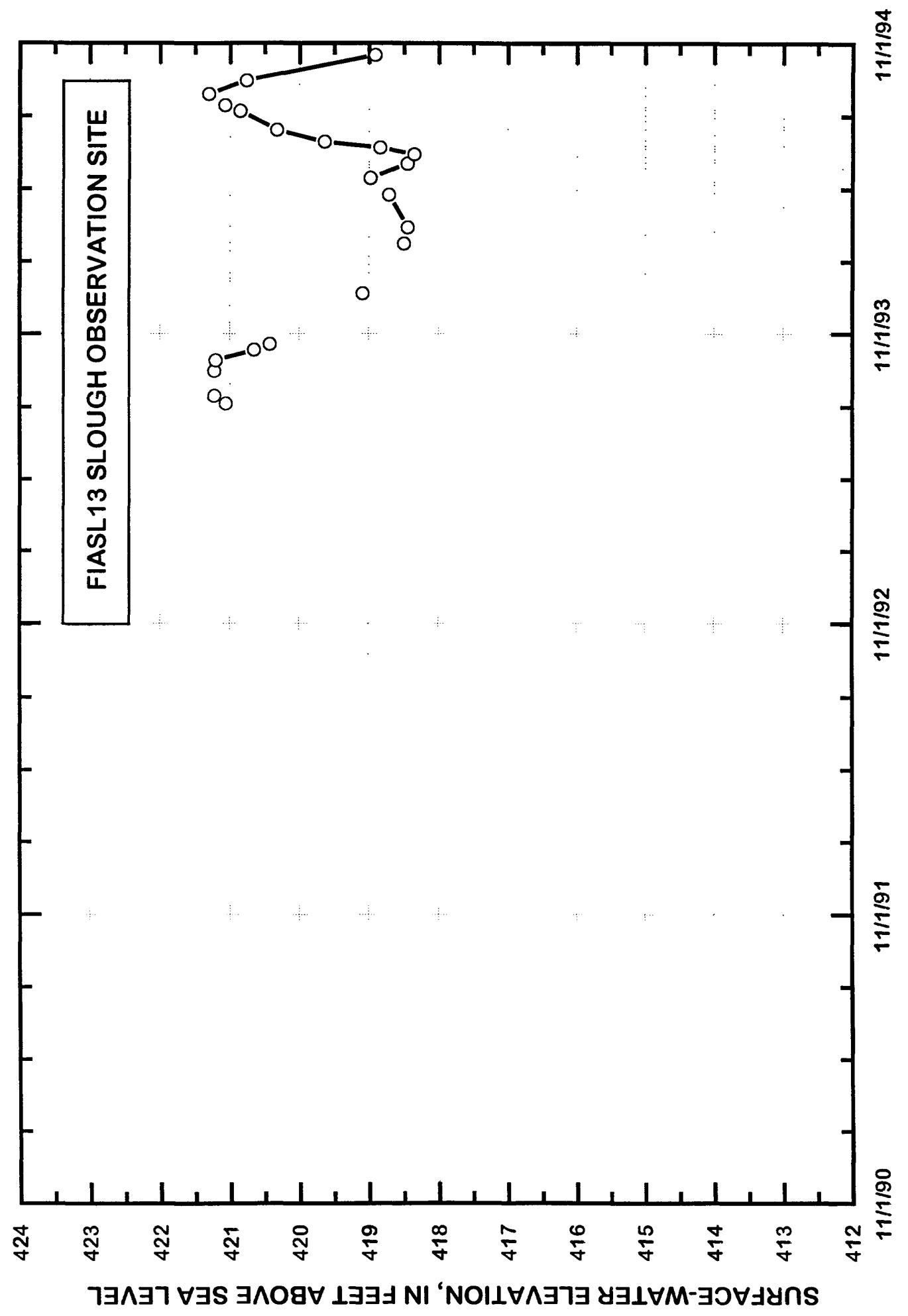



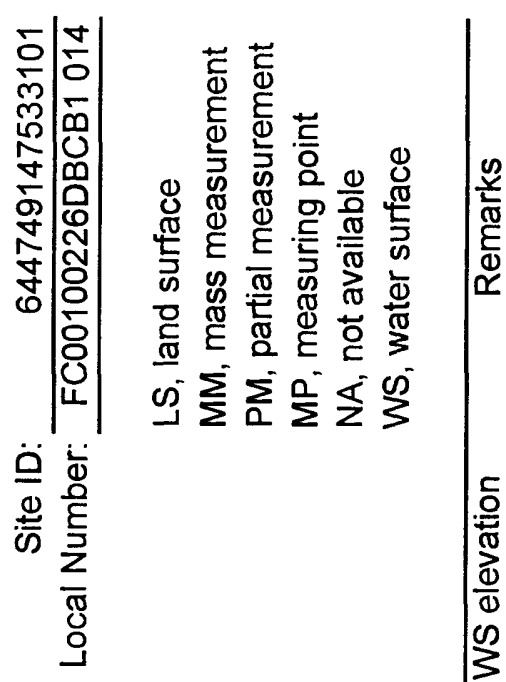

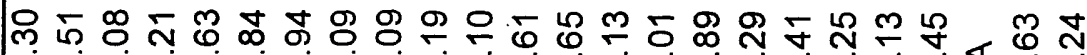

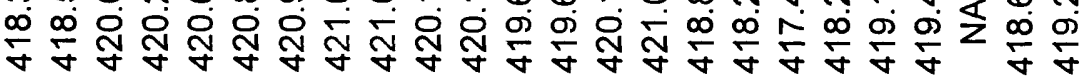

$\infty$

3 c

(3)

产政

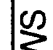

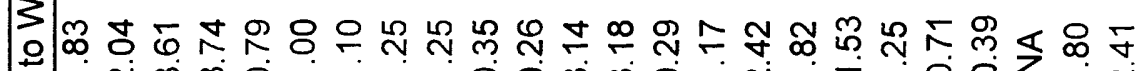
$\sum$

rim m

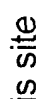

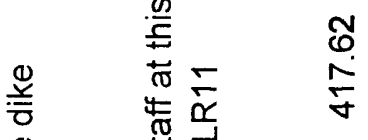

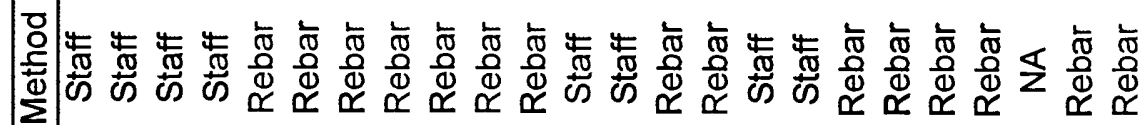

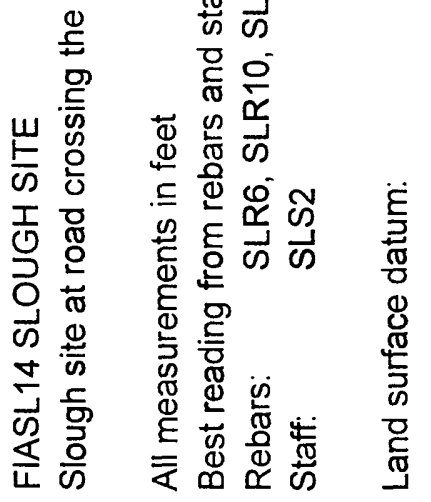

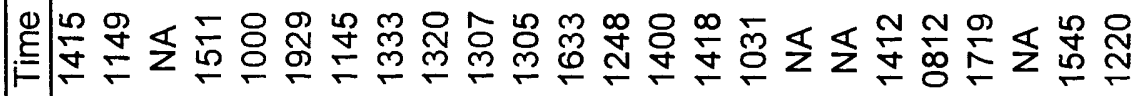

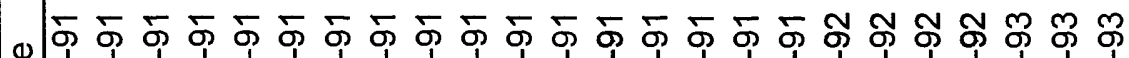

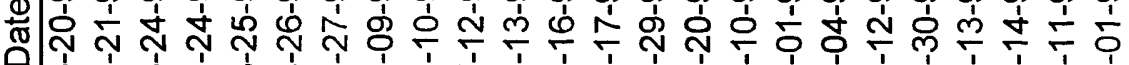
ஜ́ 


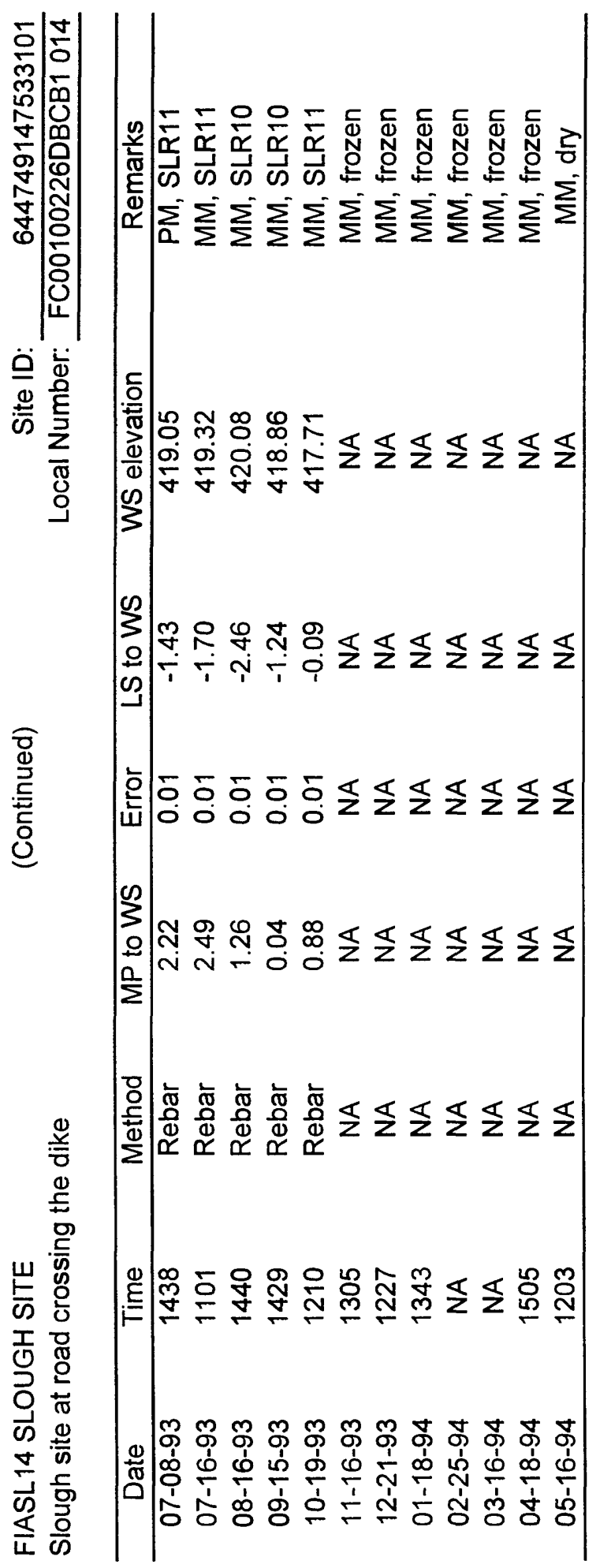




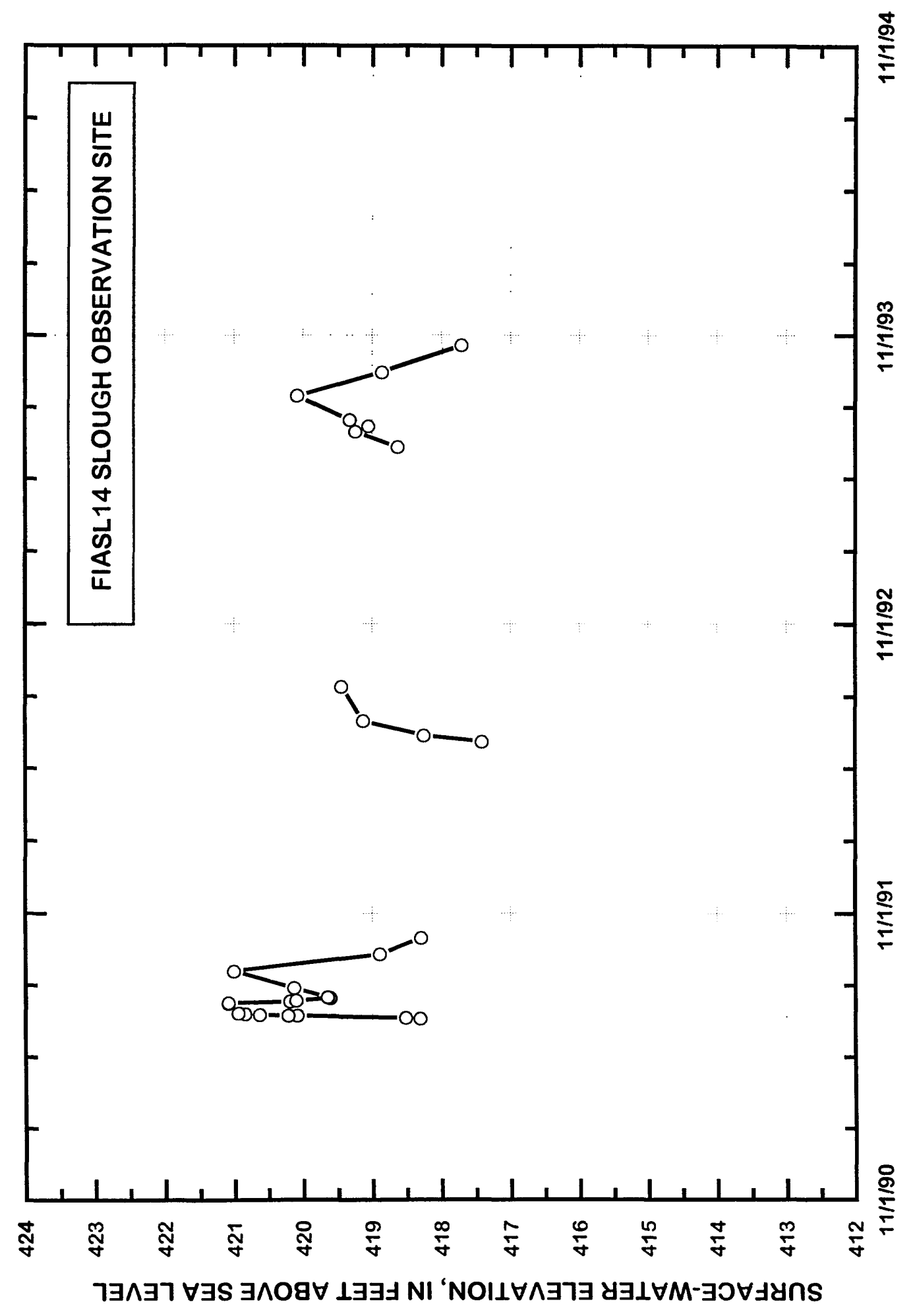



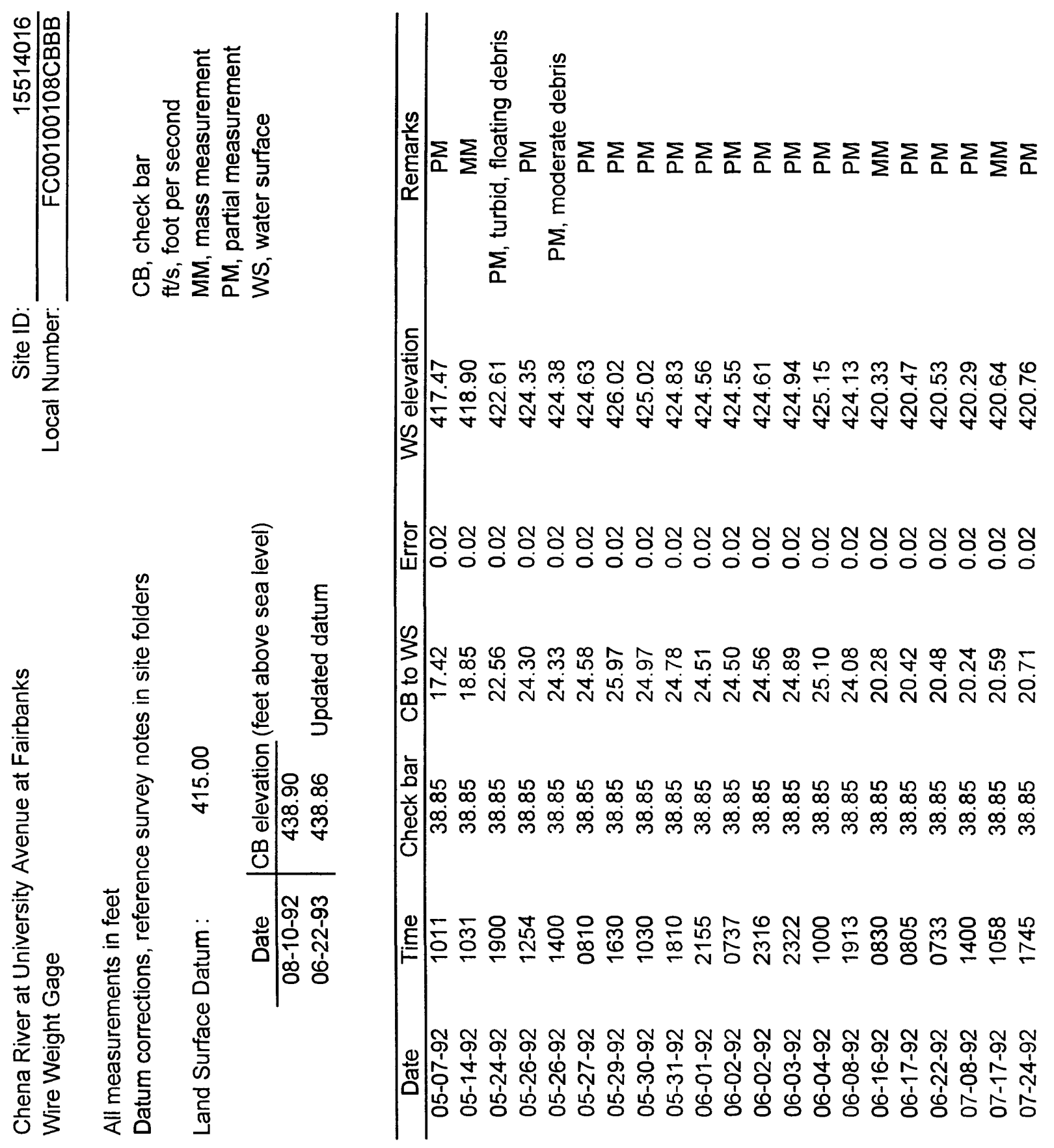


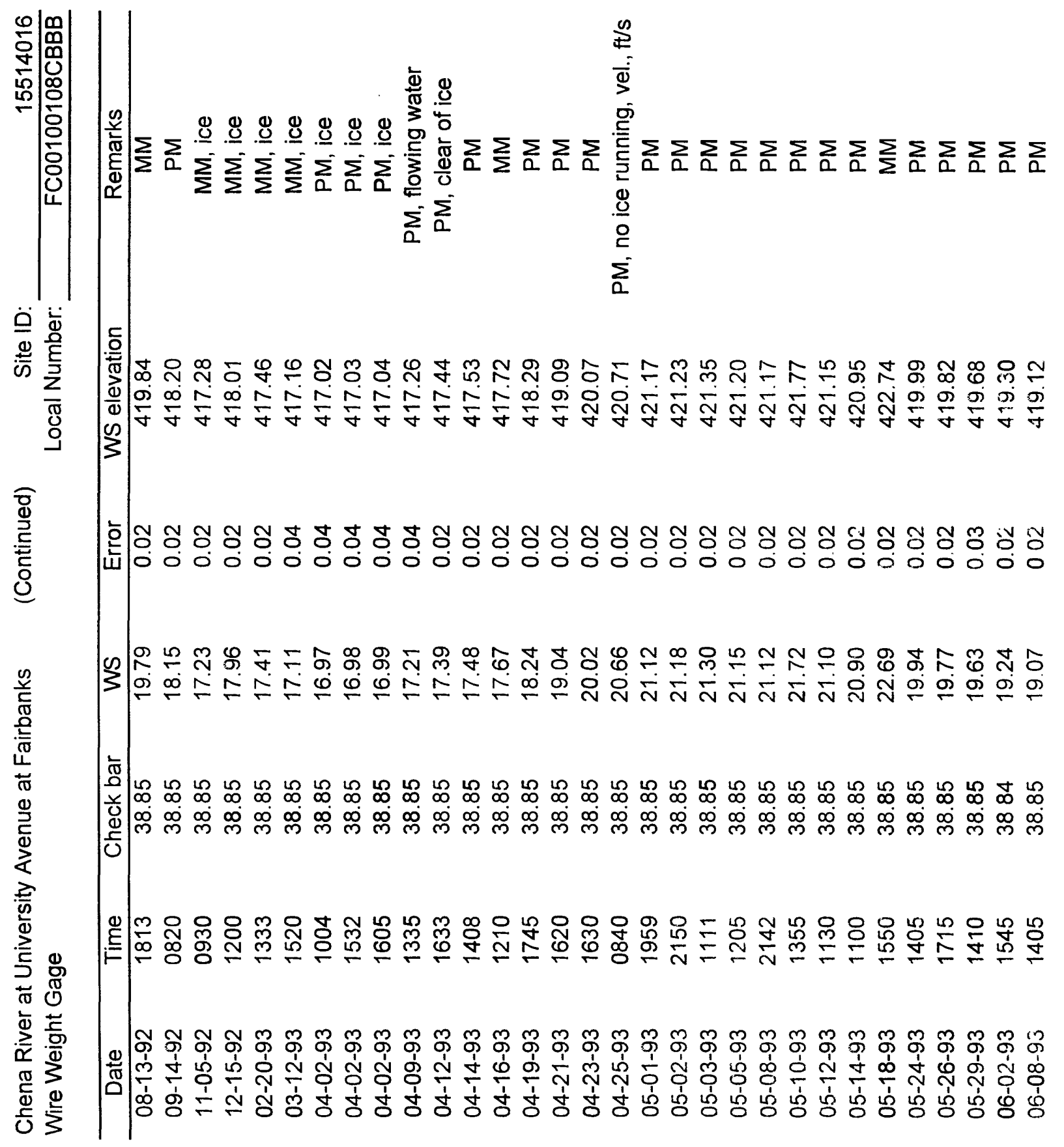




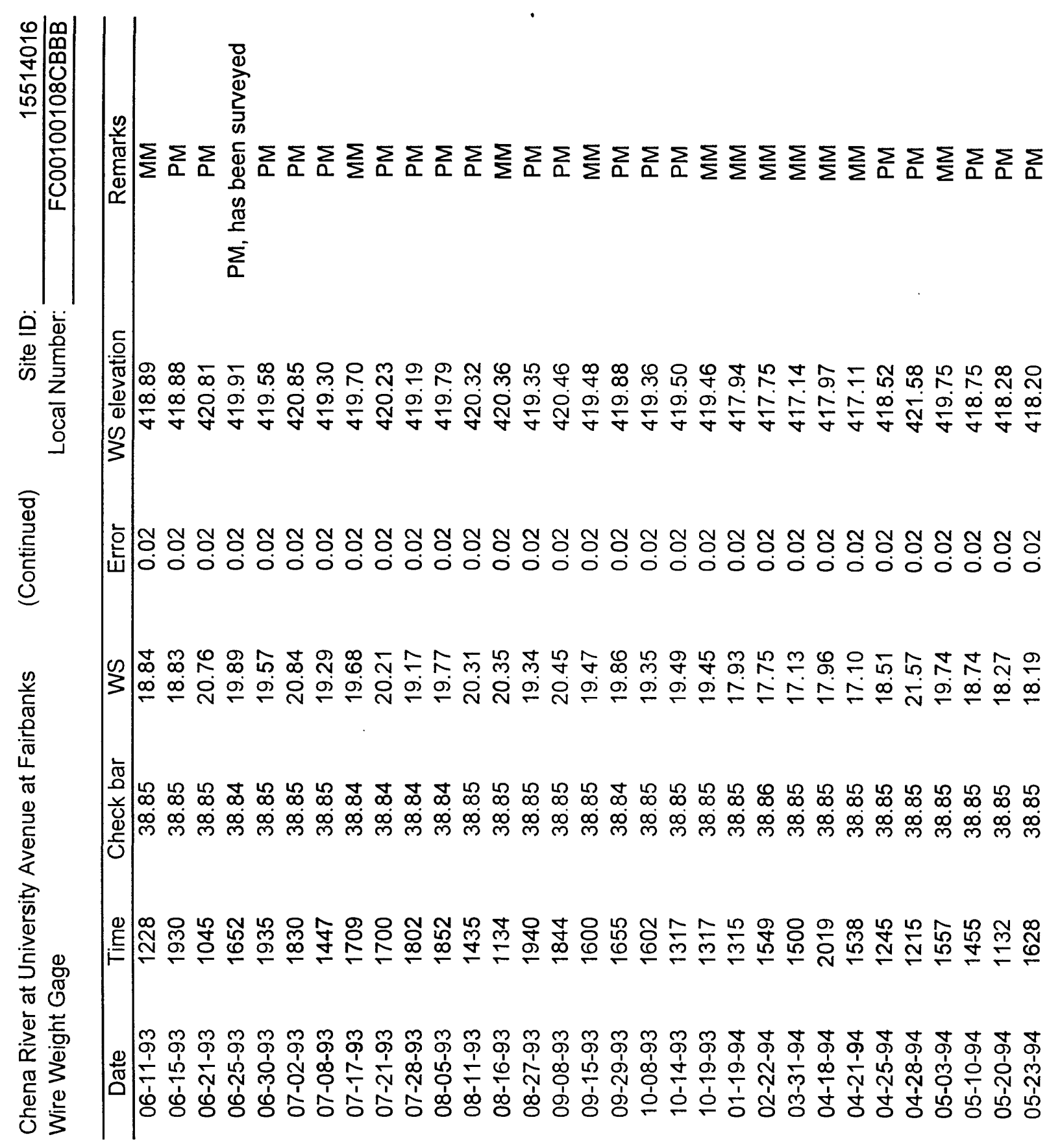




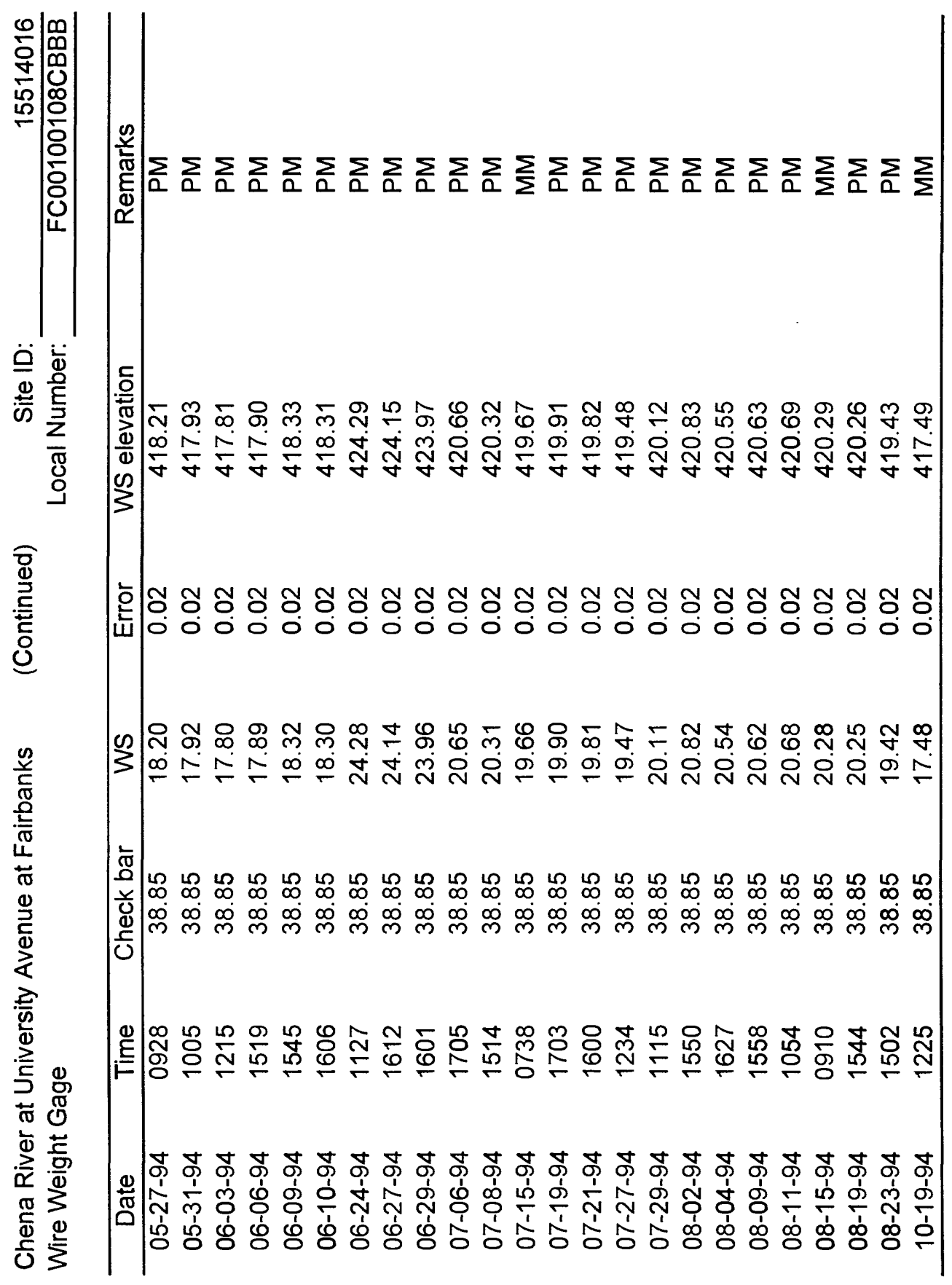




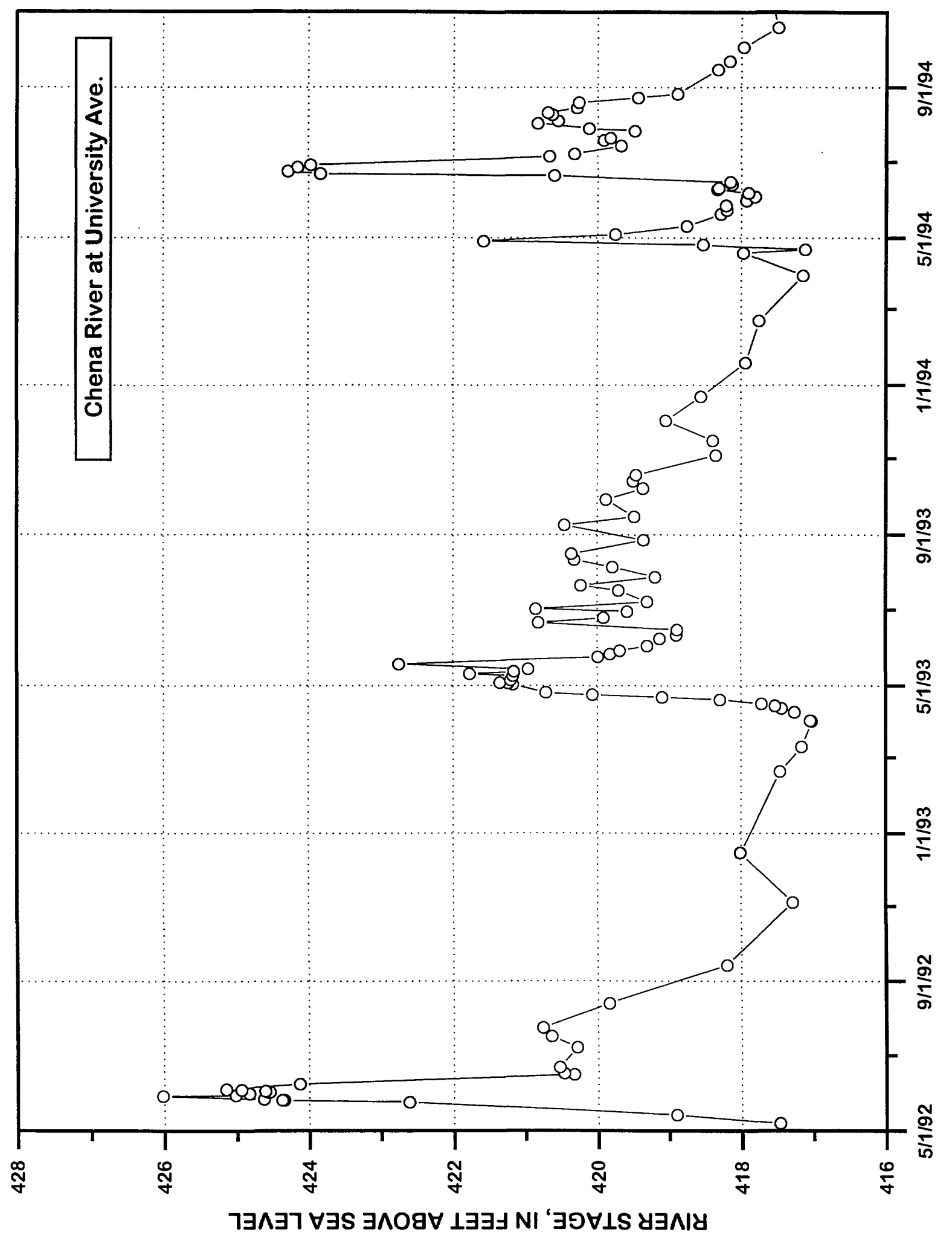



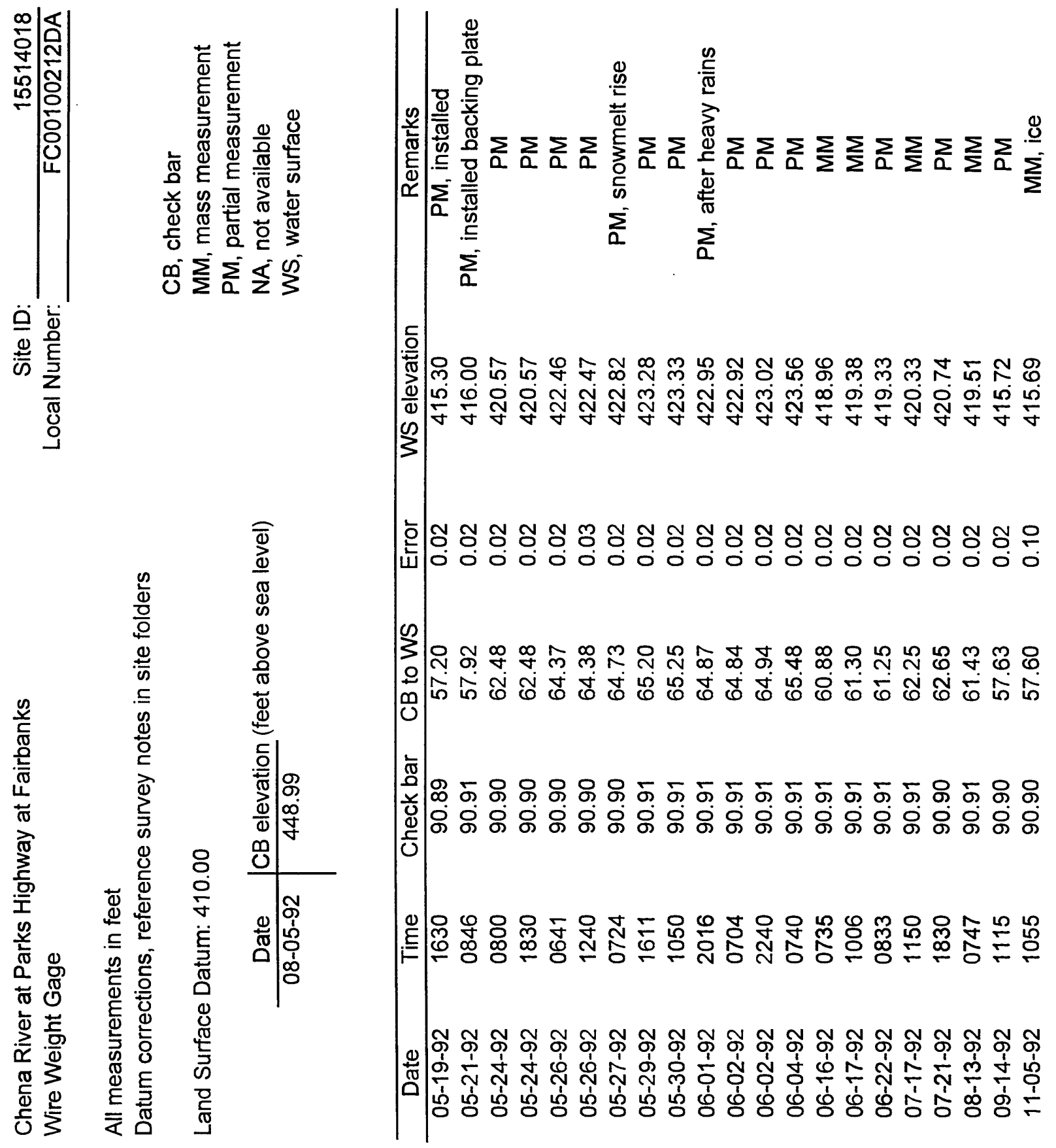


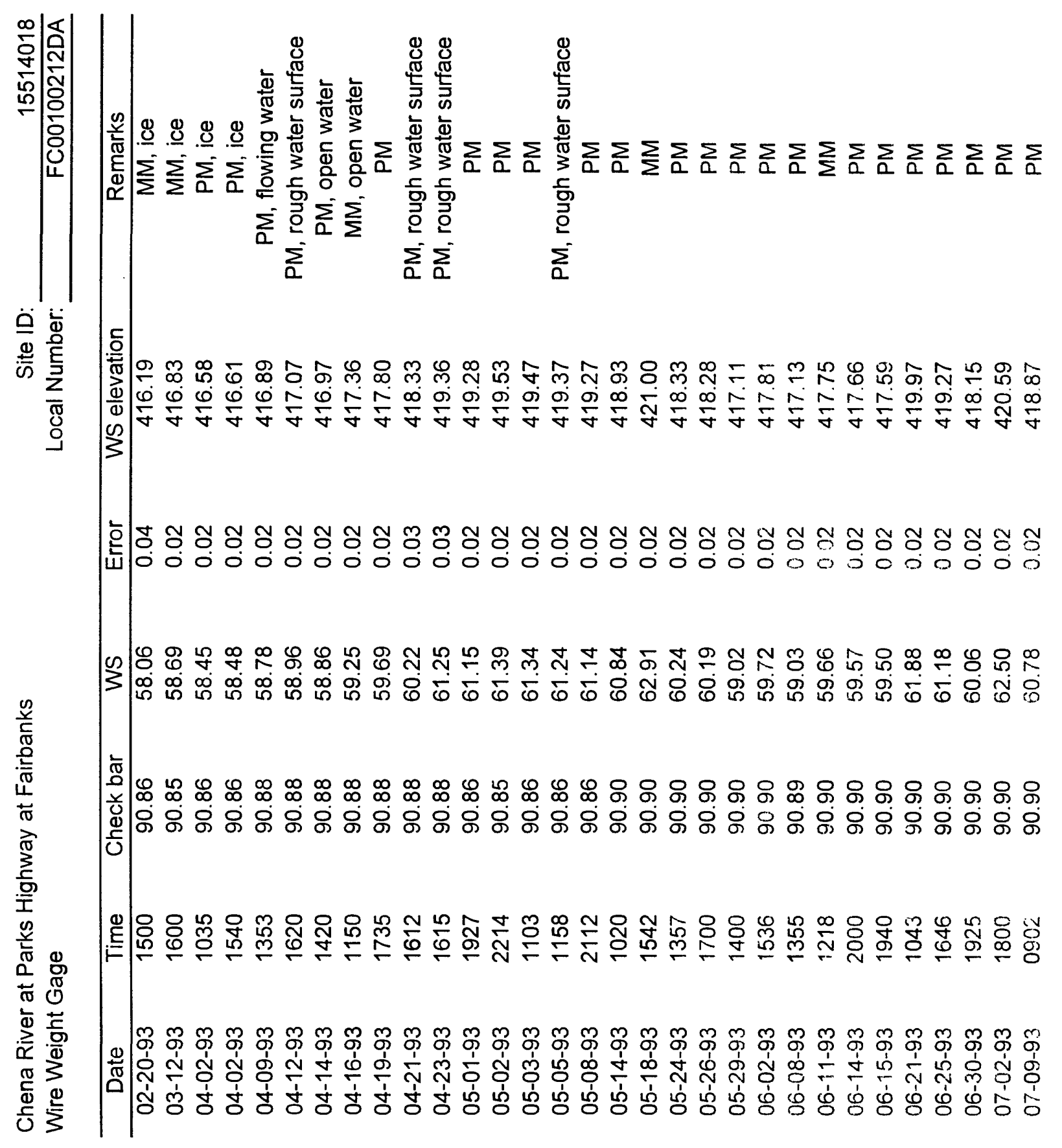




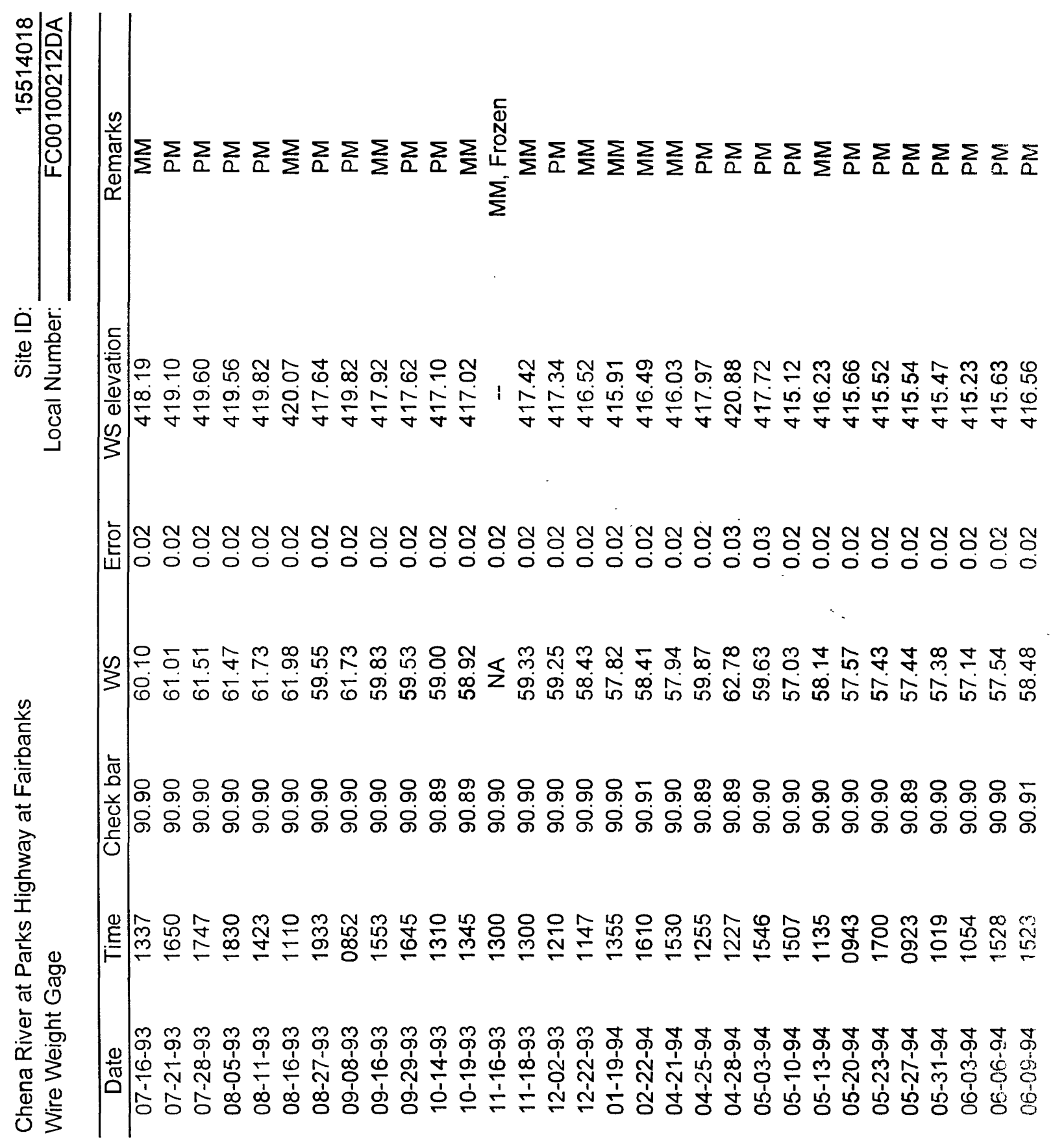




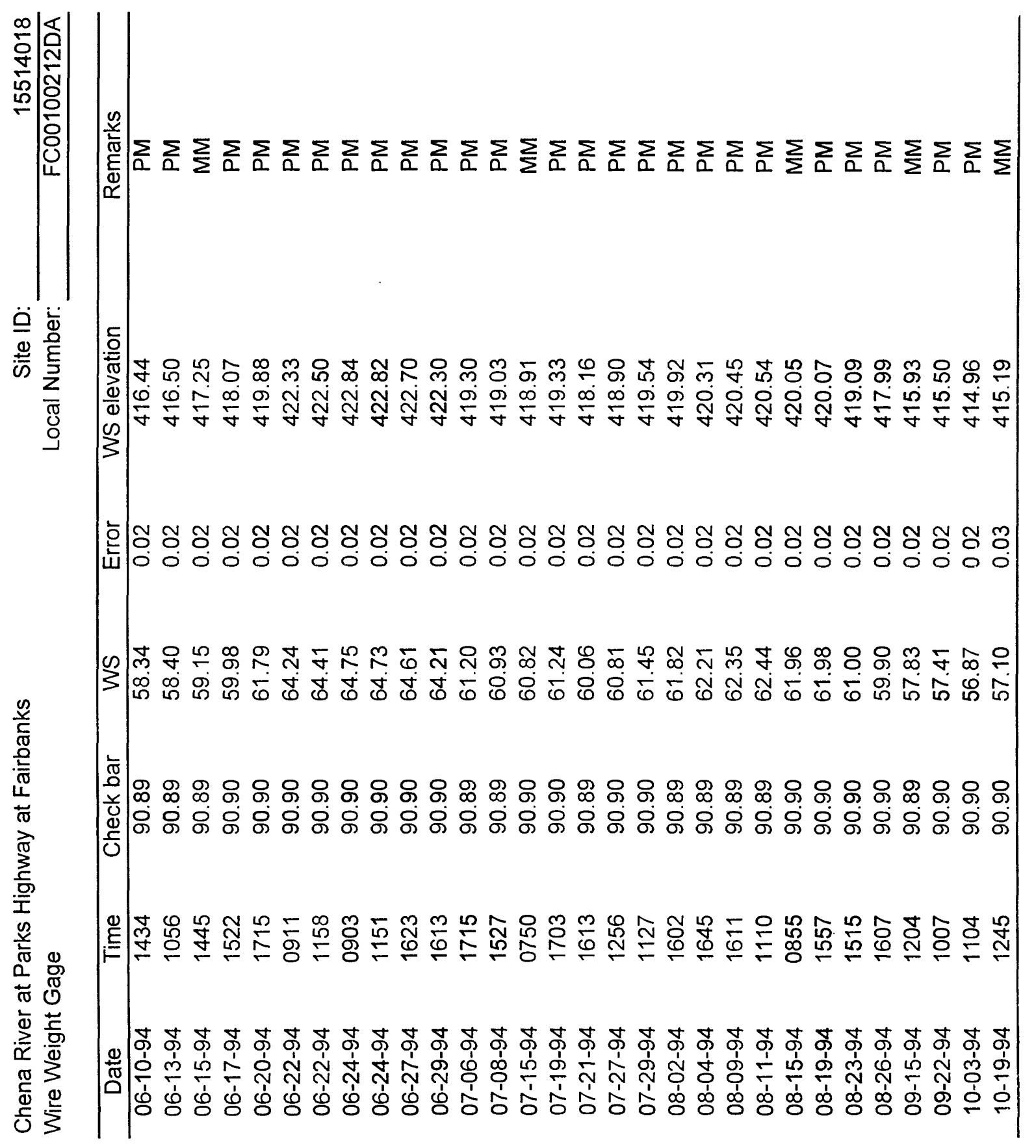




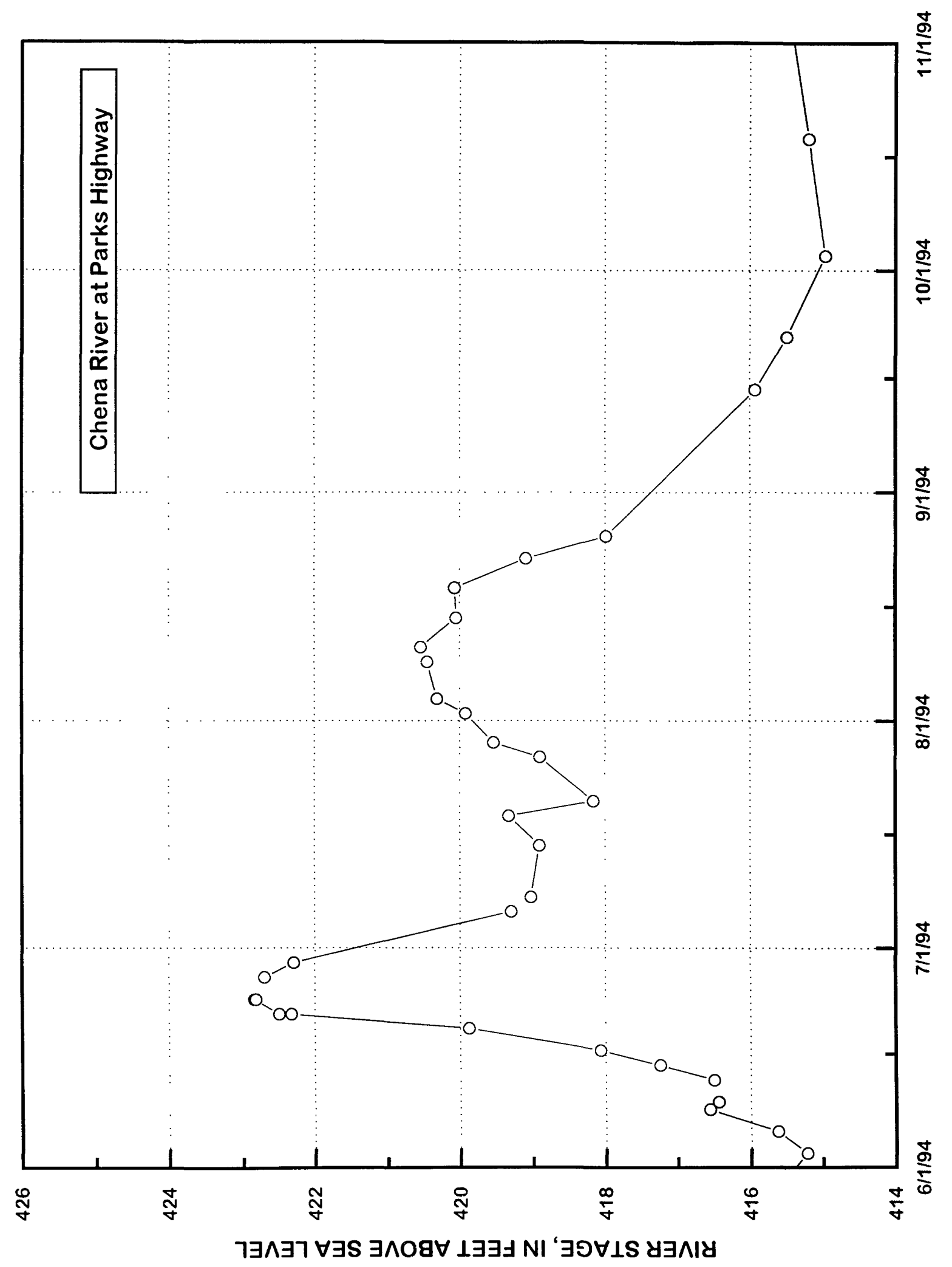



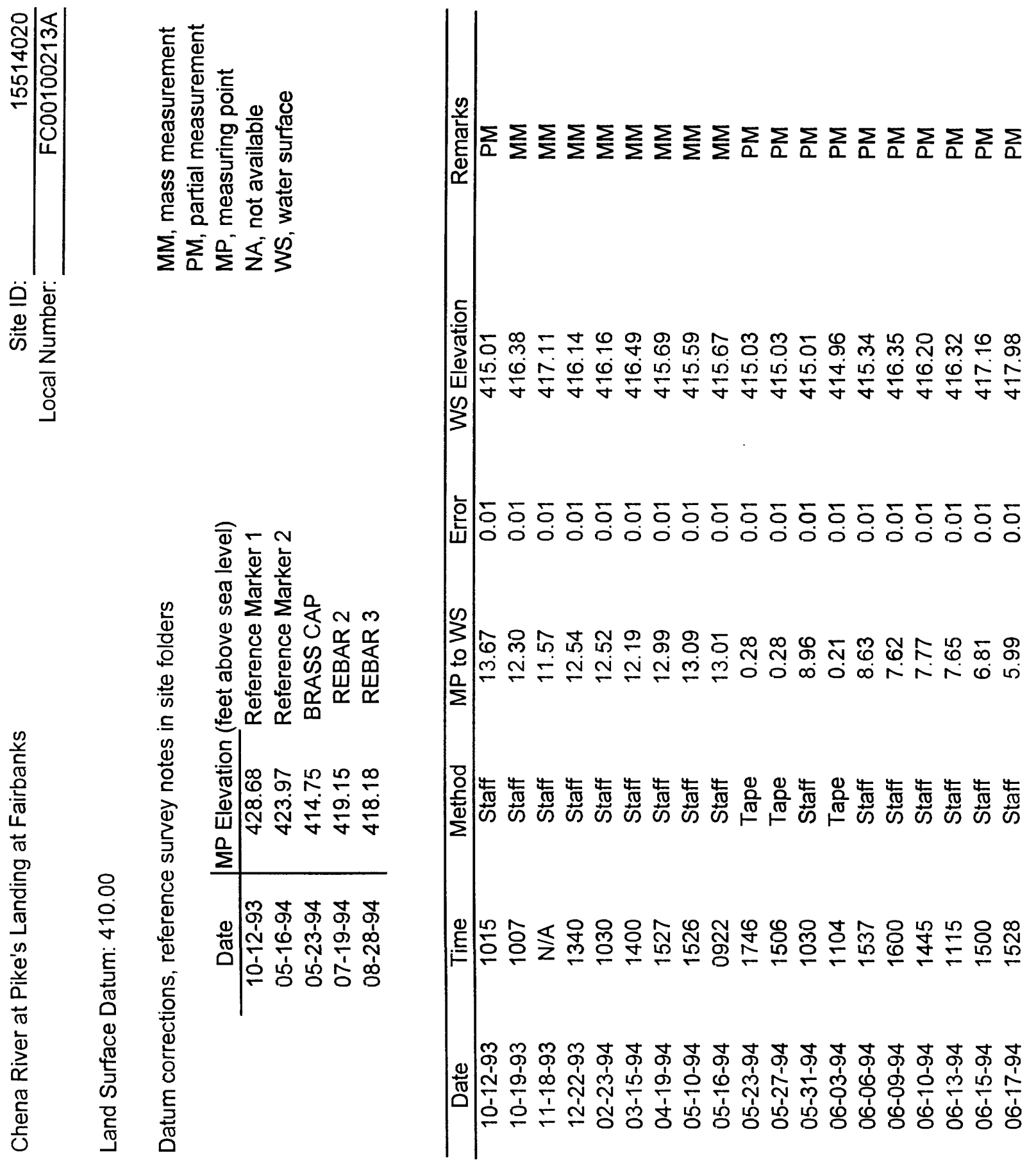


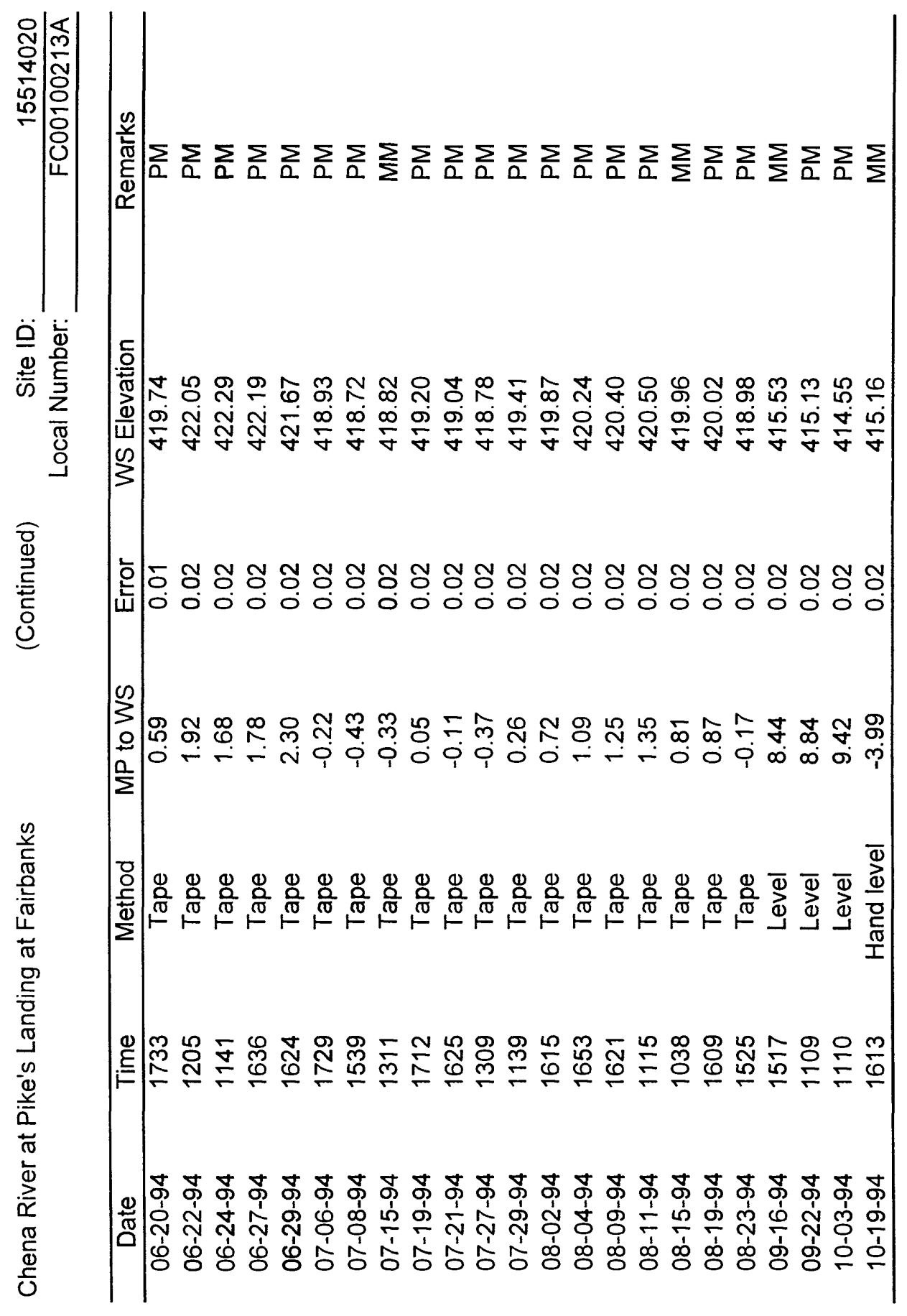




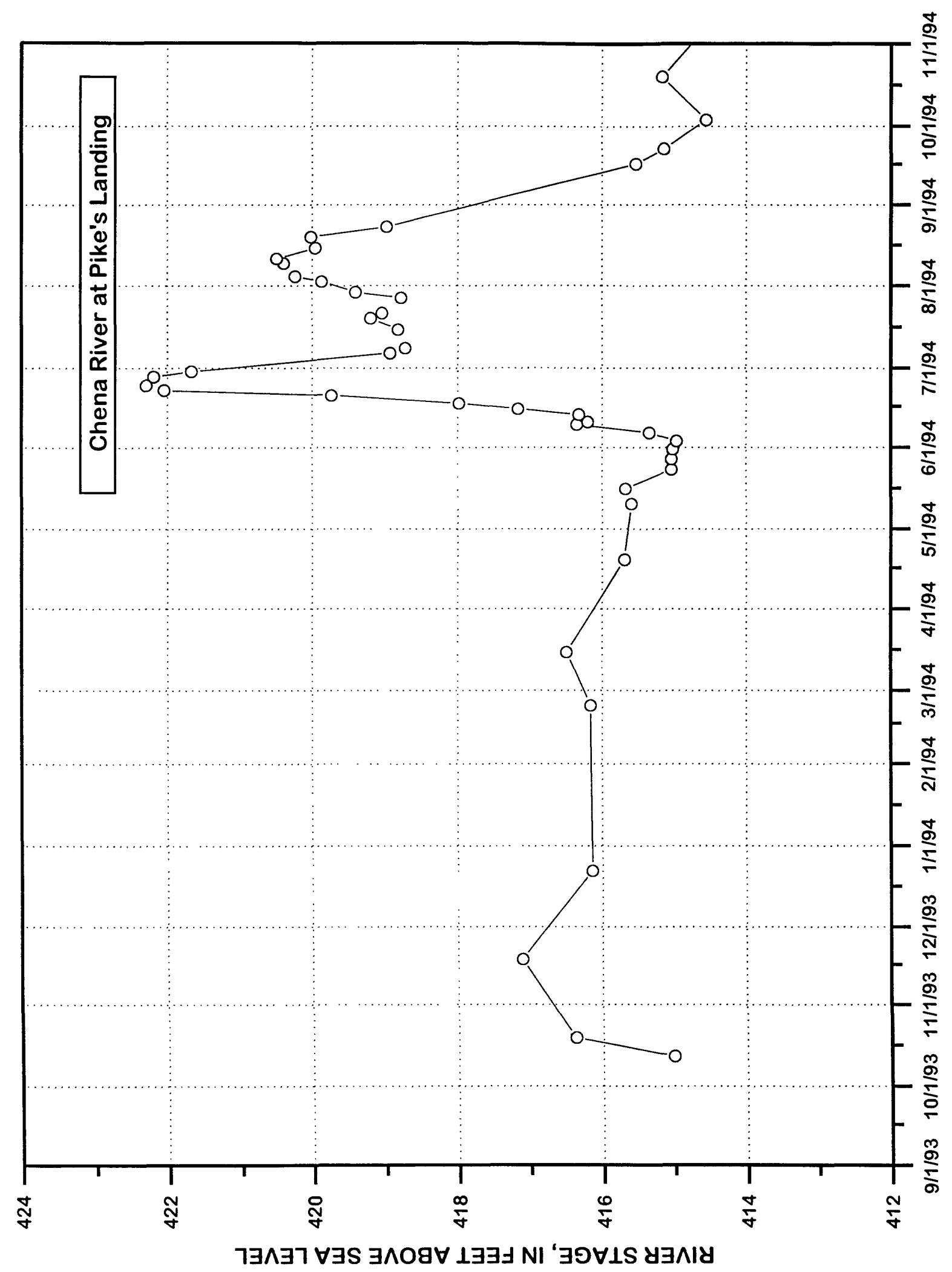




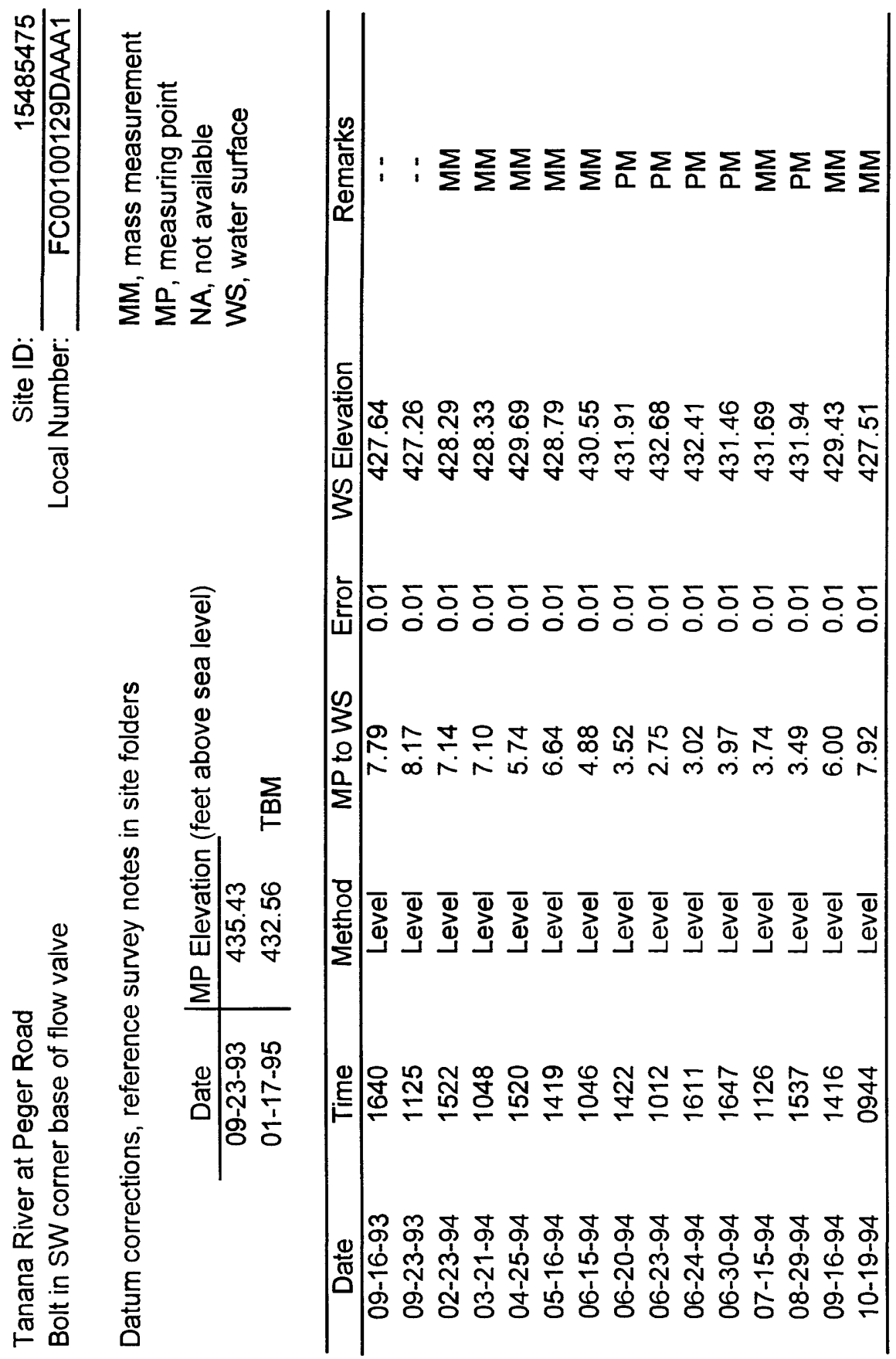




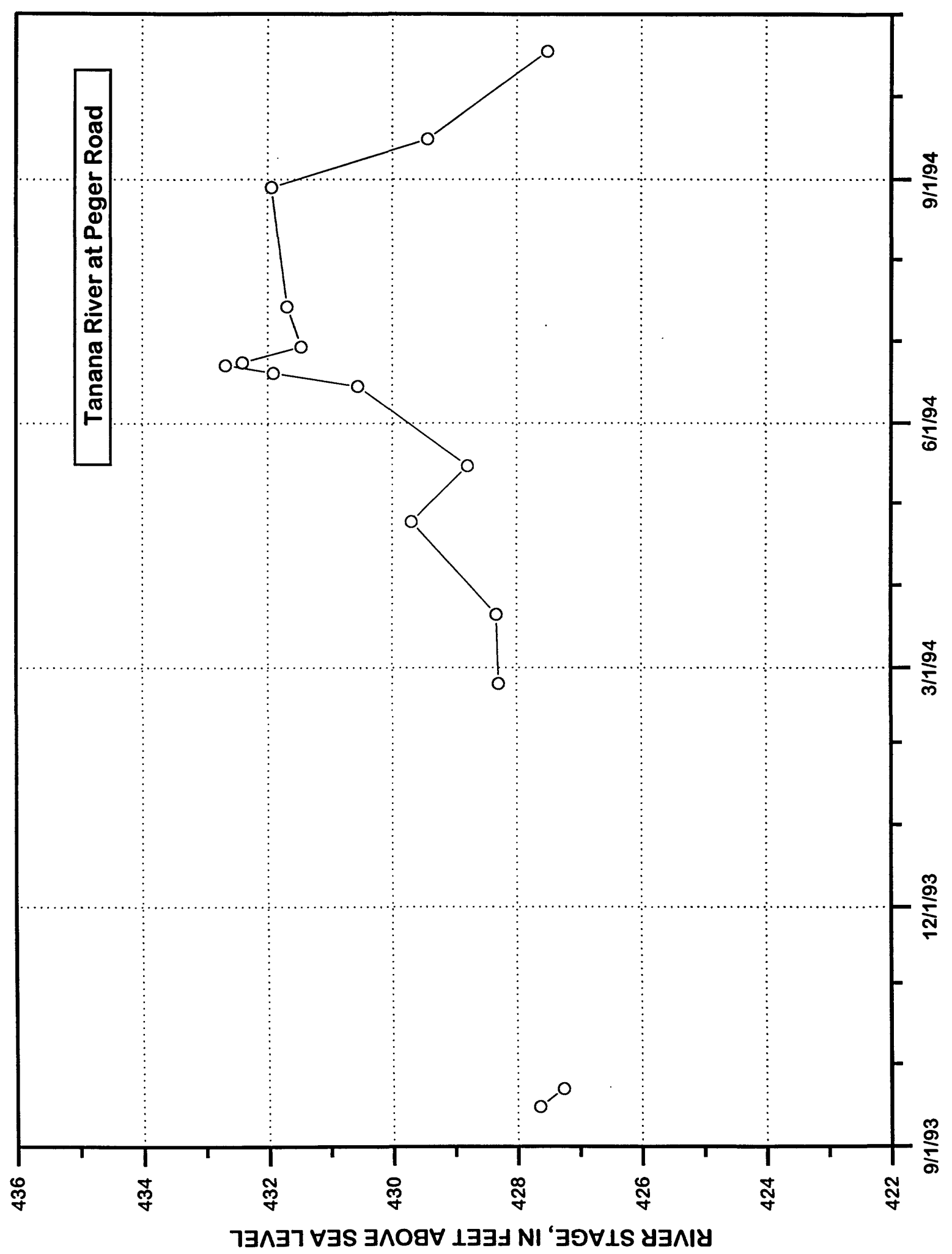

